FERMILAB-PUB-11-195-T

OUTP-11-39P

\title{
One-loop calculations in quantum field theory: from Feynman diagrams to unitarity cuts
}

\author{
R. Keith Ellis ${ }^{\mathrm{a}}$, Zoltan Kunszt ${ }^{\mathrm{b}}$, Kirill Melnikov ${ }^{\mathrm{c}}$, Giulia Zanderighi ${ }^{\mathrm{d}}$ \\ ${ }^{a}$ Fermilab, Batavia, IL 60510, USA \\ ${ }^{b}$ Institute for Theoretical Physics, ETH, Zurich, CH-8093 Zurich, Switzerland \\ ${ }^{c}$ Department of Physics, Johns Hopkins University, Baltimore, USA \\ ${ }^{d}$ Rudolf Peierls Centre for Theoretical Physics, 1 Keble Road, University of Oxford, UK
}

\begin{abstract}
The success of the experimental program at the Tevatron re-inforced the idea that precision physics at hadron colliders is desirable and, indeed, possible. The Tevatron data strongly suggests that one-loop computations in QCD describe hard scattering well. Extrapolating this observation to the LHC, we conclude that knowledge of many short-distance processes at next-to-leading order may be required to describe the physics of hard scattering. While the field of oneloop computations is quite mature, parton multiplicities in hard LHC events are so high that traditional computational techniques become inefficient. Recently new approaches based on unitarity have been developed for calculating one-loop scattering amplitudes in quantum field theory. These methods are especially suitable for the description of multi-particle processes in QCD and are amenable to numerical implementations. We present a systematic pedagogical description of both conceptual and technical aspects of the new methods.
\end{abstract}

Keywords: QCD, perturbation theory, helicity amplitudes, precision calculations, LHC

PACS: 14.60.Ef,13.40.Em

Email addresses: ellis@fnal.gov (R. Keith Ellis), kunszt@itp.phys.ethz.ch (Zoltan Kunszt), melnikov@pha.jhu.edu (Kirill Melnikov), g.zanderighi1@physics.ox.ac.uk (Giulia Zanderighi) 


\section{Contents}

1 Introduction $\quad 4$

2 One loop diagrams: the traditional approach $\quad 7$

2.1 Preliminary remarks ................. . . 7

2.2 One-loop scalar integrals . . . . . . . . . . . . . . . 10

2.3 One-loop tensor integrals and form factor expansion . . . . . . . 10

2.4 Singular regions . . . . . . . . . . . . . . . . . . . 14

2.5 Advanced diagrammatic methods . . . . . . . . . . 15

3 Van Neerven - Vermaseren basis 17

3.1 The Van Neerven - Vermaseren basis . . . . . . . . . . . . . . . 17

3.2 Physical and transverse space . . . . . . . . . . . . . . . 19

4 Reduction at the integrand level in two dimensions 24

4.1 Reduction of a scalar triangle . . . . . . . . . . . . . . . 24

4.2 Reduction of a rank-two two-point function . . . . . . . . . . . 26

4.3 The photon mass in the Schwinger model . . . . . . . . . . . . . 29

5 Reduction at the integrand level in $D$-dimensions 31

5.1 Parametrization of the integrand . . . . . . . . . . 31

5.2 Computation of the reduction coefficients . . . . . . . . . . 36

5.3 Comments on the rational part . . . . . . . . . . . . . . . 44

5.4 Rational terms by Passarino-Veltman reduction . . . . . . . . . . 47

6 Managing the color $\quad 48$

$6.1 n$-gluon amplitudes . . . . . . . . . . . . . . . . . . . . . 48

$6.2 \bar{q} q+(n-2)$-gluon amplitudes . . . . . . . . . . . . . 56

6.3 Amplitudes with multiple quark pairs . . . . . . . . . . . 61

6.4 Singular behavior of one-loop amplitudes . . . . . . . . . 66

7 Colorless amplitudes $\quad \mathbf{7 2}$

7.1 Colorless loop integrand in $D$-dimensions . . . . . . . . . . . . . 72

7.2 Polarization states in $D$-dimensions . . . . . . . . . . . . . . . . 75

7.3 Berends-Giele recursion relations . . . . . . . . . . . . . 81

7.4 Britto-Cachazo-Feng-Witten relations for on-shell amplitudes . . 84

7.5 Computations with massive particles . . . . . . . . . 88

8 Analytic computations $\quad 91$

8.1 Direct computation of the cut-constructible coefficients . . . . . . 91

8.2 An alternative formula for bubble reduction coefficients . . . . . 101

8.3 Direct computation of the rational part . . . . . . . . . . . 105

8.4 Using the helicity formalism . . . . . . . . . . . . . . 108 
9 Examples 108

9.1 Higgs decay to two photons through massless scalars . . . . . . . 109

9.2 Higgs decay to two gluons through massive quarks loop . . . . . 111

9.3 The rational part of the four-photon scattering amplitudes . . . . 115

9.4 Rational parts of $n$-photon scattering amplitudes, $n>4$. . . 120

9.5 The axial anomaly . . . . . . . . . . . . . . 122

9.6 Calculation of one-loop corrections to $\bar{q} q g g$. . . . . . . . . . 126

10 Numerical implementation $\quad \mathbf{1 3 5}$

10.1 Comments on the numerical implementation . . . . . . . . . . 135

10.2 Checks and numerical instabilities . . . . . . . . . . . . . 138

10.3 Time dependence of the $D$-dimensional unitarity algorithm . . . 141

11 Outlook

Appendix A Details of Passarino-Veltman decomposition 147

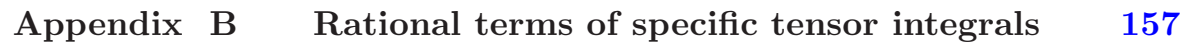

$\begin{array}{lll}\text { Appendix C } & \text { Cutkosky rules }\end{array}$

Appendix D Spinor Helicity methods 165

Appendix D.1 Spinor solutions for massless fermions . . . . . . . 165

Appendix D.2 Spinor products . . . . . . . . . . . 166

$\begin{array}{llll}\text { Appendix } & \mathrm{E} & \text { Results for selected scalar integrals } & 171\end{array}$

Bibliography

172 


\section{Introduction}

Perturbation theory is one of the few rigorous ways to connect Quantum Chromodynamics (QCD) to observations. It is particularly important because, during the next decade, our understanding of particle physics will be challenged by experiments at the Large Hadron Collider (LHC) at CERN. Hadron collisions at large momentum transfer are important for the direct observation of new forces and new forms of matter. Thanks to the phenomenon of asymptotic freedom in QCD, the strong coupling constant becomes small at large momentum transfer, making it possible to describe hard hadron collisions in QCD perturbation theory.

During the past decade, both Tevatron experiments - CDF and D0 - have accumulated an enormous luminosity, of the order of ten inverse femtobarns. This high luminosity enabled detailed and careful investigation of Standard Model processes, including jets and electroweak gauge boson production as well as studies of the top quark and the Higgs boson. In all the cases considered, the comparison of observables that are calculable in perturbative QCD with experimental results improved if next-to-leading order (NLO) QCD computations were used. This fact establishes perturbative QCD as a systematic framework to describe the physics of hard hadronic collisions. It also suggests that, ideally, the theoretical toolkit for the LHC should contain next-to-leading computations for a large variety of processes.

This idea was formalized by the so-called wishlist which puts together a collection of processes whose computation through NLO QCD is thought to be most useful [1]. The list includes large number of processes with three or four particles in the final state; those final-state particles include QCD partons, heavy quarks and electroweak gauge bosons. The wishlist was originally compiled back in 2003 and, at that time, the computation of even the simplest processes on the wishlist was considered to be very challenging.

The situation changed dramatically in the past four to five years since new techniques for one-loop computations lead to the explosion of new results in the field. In the past three years, a large number of one-loop computations with four (massive and massless) particles in the final state were completed $[2,3,4,5,6,7,8,9,10,11,12,13,14,15,16]$ and even the first $2 \rightarrow 5$ results the NLO QCD corrections to $p p \rightarrow W(Z)+4 j[17,18]$ - was obtained. ${ }^{1}$ Some of this progress is to be attributed to spectacular improvements of the existing Feynman-diagrammatic algorithms for one-loop calculations, whose efficiency and stability was boosted beyond expectations. Furthermore, the past three years have witnessed a full development of the idea that one-loop amplitudes can be reconstructed from their unitarity cuts. This idea was put forward by Bern, Dixon and Kosower in the 1990s and was used in a number of phenomenological calculations [19]. It was revived in recent years when Britto, Cachazo and Feng observed that the coefficients of the four-point functions, obtained

\footnotetext{
${ }^{1}$ As usual, we do not include decay products of heavy particles in this counting.
} 
when one-loop amplitudes are reduced to scalar integrals, are products of onshell tree scattering amplitudes evaluated at complex momenta [20], and when Ossola, Papadopoulos and Pittau (OPP) discovered a simple algebraic method for reducing tensor integrals to scalar master integrals [21].

In this article we describe these developments using the technique developed by some of us, in collaboration with W. Giele, that we will refer to as the generalized $D$-dimensional unitarity $[22,23,24]$. In contrast to other unitarity-related techniques, this is the only method known today that delivers complete oneloop scattering amplitudes in renormalizable quantum field theories, regardless of whether one-loop amplitudes involve massless or massive particles.

The goal of this review is to present ideas and techniques of generalized $D$-dimensional unitarity in a pedagogical manner. Although a fair number of advanced one-loop calculations has been already performed using this method, the subject will benefit from a detailed and critical review that will make it accessible also to a non-expert audience. While we believe that essential details of the unitarity methods are well-established by now, further algorithmic improvements are not to be excluded. Our goal therefore is to provide detailed information about the method of generalized $D$-dimensional unitarity with the hope that it can be used as a foundation for further development of this approach. We also describe some aspects of other unitarity-based techniques to one-loop computations. A more detailed description of these alternative approaches and many references to original publications can be found in recent reviews $[25,26]$. The remainder of this article is organized as follows.

In Section 2, we summarize the traditional approaches to one-loop computations. Such traditional approaches often employ the Passarino-Veltman procedure to express tensor one-loop integrals through scalar integrals. For reference purposes, we present full details of the Passarino-Veltman reduction procedure in Appendix A.

In Section 3 we introduce the van Neerven - Vermaseren basis which is important for the explicit implementation of the unitarity technique. Subsequent sections refer back to and use various results obtained in Section 3.

In Section 4 we discuss the reduction algorithm in detail by considering two-dimensional examples. Working in two-dimensional space-time offers clear advantages since it allows us to derive concise analytic results. We make use of the van Neerven - Vermaseren basis to reduce a scalar triangle to a sum of scalar two-point functions. This derivation generalizes to higher dimensions leading to the important result that any $N$-point function in $D$ dimensions, with $N>D$, can be written as a combination of $D$-point functions. We also discuss the reduction of the rank two tensor two-point function in two dimensions in two different ways. The first method employs a decomposition of the loop momentum using the van Neerven - Vermaseren basis. The second method shows that the reduction can be performed if the integrand is known at special values of the loop momentum, namely the ones for which the inverse Feynman propagators vanish. We use this example to introduce the connection between reduction procedures and the OPP/unitarity ideas [21]. We finish this Section with an example where we compute the so-called rational part - a remnant 
of the ultraviolet regularization - of the photon vacuum polarization in twodimensional QED. This contribution is responsible for the dynamical generation of the photon mass in two-dimensional QED - a remarkable phenomenon first pointed out by J. Schwinger [27].

In Section 5 we describe the explicit construction of the OPP reduction procedure in $D$-dimensions. We give full details about the parametrization of the integrand, we describe how discrete Fourier transforms can be used to extract reduction coefficients, and we show how specific situations, that could give rise to numerical instabilities, can be handled. Finally, we discuss the rational part, explain its ultraviolet origin and point out that ultraviolet finite integrals can have a rational part. In Appendix B we give the explicit expression for the rational part of specific tensor integrals used here.

In Section 6 we discuss the color decomposition of one-loop amplitudes and introduce the concept of color-ordered amplitudes. We discuss specific examples involving only gluons, one quark-pair and many gluons, as well as the case of amplitudes involving multiple quark pairs.

In Section 7 we explain how the OPP procedure can be related to unitarity and describe various ingredients that are important for a practical implementation of the computational algorithm. We present the construction of polarization states and spinors in higher dimensions. We explain how tree-level amplitudes can be computed using recursive Berends-Giele relations [28] and present examples of the recursive equations for amplitudes with up to four fermions and an arbitrary number of gluons. We also show how Britto-Cachazo-FengWitten (BCFW) relations between scattering amplitudes can be proven using Berends-Giele recursions, and that the BCFW relation is independent of the number of dimensions. Finally, we discuss subtleties related to the implementation of $D$-dimensional unitarity methods for calculating scattering amplitudes with massive particles.

In Section 8 we describe several methods of more analytic nature that are closely related to the OPP method and generalized unitarity. We give an extensive discussion of the method suggested by Forde which allows the direct computation of the cut-constructible reduction coefficients [29]. We describe a generalization of this method, suggested by Badger [30], that enables the calculation of the rational part. We also discuss a technique suggested by Mastrolia for the computation of the double-cut reduction coefficient [31]. One aspect of our discussion that makes it different from much of the literature is that we avoid the extensive use of spinor-helicity decomposition of the loop momentum and show that the analytic approaches described in Refs. [29, 30, 31] can be understood using a simple parametrization of the loop momentum phase-space in terms of polar and azimuthal angles.

In Section 9 we present three examples that emphasize the anomalous nature of the rational part. We discuss the decay of the Higgs boson to two photons through a loop of massless scalars. We demonstrate that the rational part of the photon-photon scattering amplitude is independent of the mass of the virtual particle that mediates the photon-photon scattering, and discuss the absence of the rational part in $n$-photon scattering amplitudes for $n \geq 6$, following Ref. [32]. 
Thereafter, we show that the rational part of the one-loop triangle amplitude gives the anomalous part of the divergence of the axial current. We conclude this Section with a simple example of the analytic calculation of a one-loop diagram using the spinor helicity method. A brief introduction to the spinor helicity method is given in Appendix D.

In Section 10 we describe how generalized unitarity can be implemented in a computer code. We contrast the application of the OPP reduction technique to Feynman diagrams with the unitarity-based implementation. We explain a convenient method to handle cuts in parent diagrams systematically. We discuss issues related to having colorless (unordered) particles in the scattering process. We also present the standard checks that are done on numerical calculations and issues related to numerical instabilities. Finally, we show that the computational time for one-loop amplitudes depends on the number of external particles in a polynomial way and give a few examples.

We conclude in Section 11. As a final remark we note that other approaches to one-loop calculations beyond traditional Passarino-Veltman reduction and generalized unitarity have have been studied in the literature $[33,34,35,36,37$, $38,39,40,41,42,43,44]$, but we will not discuss them in this review.

\section{One loop diagrams: the traditional approach}

\subsection{Preliminary remarks}

In this Section, we review traditional approaches to the computation of oneloop integrals. While the discussion below is general, it is useful to have in mind the mathematical structure of the Standard Model of particle physics. One-loop computations in the Standard Model require calculating integrals of the following form

$$
I_{N} \sim \int \frac{\mathrm{d}^{4} l}{(2 \pi)^{4}} \frac{\mathcal{N}(l)}{\left(\left(l+q_{0}\right)^{2}-m_{1}^{2}\right)\left(\left(l+q_{1}\right)^{2}-m_{2}^{2}\right) \ldots\left(\left(l+q_{N-1}\right)^{2}-m_{N}^{2}\right)},
$$

where $N$ is the number of external particles, $q_{j}=\sum_{k=1}^{j} p_{k}$ and $p_{1}+p_{2}+\ldots . p_{N}=$ 0 thanks to the momentum conservation, see Fig. 2.1. The special case where $\mathcal{N}(l)=1$ is referred to as a scalar integral. In general $\mathcal{N}(l)$ is a polynomial function of the loop momentum $l$ as well as external momenta $p_{i}$, external polarization vectors, spinors etc. The goal is to compute $I_{N}$ in an efficient way.

Before discussing how this can be done, we point out two things in connection with the structure of $I_{N}$. First, we note that if $\mathcal{N}(l)$ contains the loop momentum $l$ in a high enough power, the integral $I_{N}$ will be ultraviolet (UV) divergent. From simple power counting, it is clear that for an $N$-point integral, the UV divergence appears if $\mathcal{N}(l)$ contains tensor integrals of rank $r$ higher than $r \geq 2 N-4$. In particular, only one-point and two-point scalar integrals are UV divergent. The highest rank of an $N$-point one-loop diagram, occurring in renormalizable quantum field theories, such as the Standard Model or QCD, is $r=N$. Hence, only one-, two-, three- and four-point one-loop integrals can be divergent in the ultraviolet region while five- and higher-point one-loop 


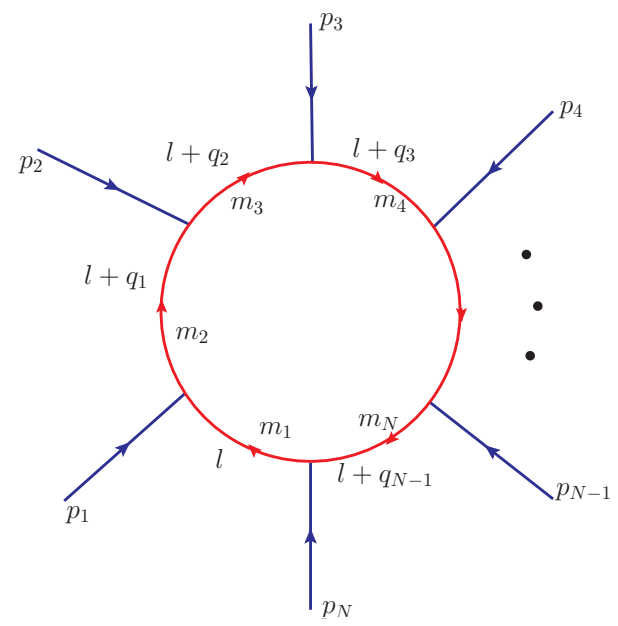

Figure 2.1: Generic diagram at one-loop with $N$ external momenta.

integrals are ultraviolet finite. In the presence of ultraviolet divergences the integrals require regularization. It is conventional to employ dimensional regularization [45], where the dimensionality of space-time is set to $D=4-2 \epsilon$, and the limit $\epsilon \rightarrow 0$ is taken at the end of the calculation. As a result, the loop momentum $l$ becomes a $D$-dimensional vector and the integration measure in Eq. (2.1) is changed to

$$
\frac{\mathrm{d}^{4} l}{(2 \pi)^{4}} \rightarrow \frac{\mathrm{d}^{D} l}{(2 \pi)^{D}}
$$

It is easy to see that this modification of the integration measure in Eq. (2.2) regularizes ultraviolet divergences. Indeed, consider as an example $I_{4}$ with $\mathcal{N}(l)=l_{\mu} l_{\nu} l_{\rho} l_{\delta}$. Power counting suggests that the divergence is logarithmic; as a consequence it is insensitive to external kinematic parameters. The divergence can be isolated by considering

$$
I_{4} \rightarrow \int \frac{\mathrm{d}^{D} l}{(2 \pi)^{D}} \frac{l_{\mu} l_{\nu} l_{\rho} l_{\delta}}{[d(l)]^{4}}=\frac{\left(g_{\mu \nu} g_{\rho \delta}+g_{\mu \rho} g_{\nu \delta}+g_{\mu \delta} g_{\nu \rho}\right)}{D(D+2)} \int \frac{\mathrm{d}^{D} l}{(2 \pi)^{D}} \frac{l^{4}}{[d(l)]^{4}},
$$

where $d(l)=l^{2}-\mu^{2}$ and $\mu$ is some kinematic invariant that we keep to regulate potential divergences at small values of $l^{2}$. The integral is calculated with the help of the following equation

$$
\begin{aligned}
& \int \frac{d^{D} l}{i \pi^{D / 2}} \frac{\left(l^{2}\right)^{r}}{\left(l^{2}-\mu^{2}\right)^{m}}=\frac{\Omega_{D}}{\pi^{D / 2}}(-1)^{r-m} \mu^{D+2 r-2 m} \int_{0}^{\infty} d x \frac{x^{2 r+D-1}}{\left(1+x^{2}\right)^{m}} \\
& =(-1)^{r-m} \mu^{D+2 r-2 m} \frac{\Gamma(r+D / 2) \Gamma(m-r-D / 2)}{\Gamma(D / 2) \Gamma(m)}
\end{aligned}
$$


where $\Omega_{D}=2 \pi^{D / 2} / \Gamma(D / 2)$ is the solid angle in $D$ dimensions. Eq. (2.4) gives a finite result for $D \neq 4$ for $m=4$ and $r=2$. We therefore conclude that dimensional regularization does indeed regularize UV divergences in one-loop computations in quantum field theories.

There is a second type of potential problem related to one-loop integrals $I_{N}$. These problems appear when a sufficient number of propagators in the integrand can go on the mass-shell simultaneously, introducing potentially nonintegrable singularities. The general theory of such singularities is provided by the Landau rules [46]. The two most important examples of these singularities are the soft and collinear ones, related to the presence of massless particles [47]. Those singularities can also be regularized dimensionally [48].

Dimensional regularization therefore provides an economical tool to define one-loop integrals in UV-divergent, renormalizable theories that contain massless particles since, with a single parameter $\epsilon=(4-D) / 2$, we are able to regulate both types of divergences and make the integrals finite. From now on, we assume that dimensional regularization is always applied to loop integrals and all the loop integrals that we have to calculate, need to be understood in that framework.

It turns out that, in the limit $D \rightarrow 4$, any integral $I_{N}$ can be written as a linear combination of one-loop scalar integrals, that include four-, three-, two- and one-point functions and a remnant of the dimensional regularization procedure that is called the rational part $\mathcal{R}$

$$
I_{N}=c_{4 ; j} I_{4 ; j}+c_{3 ; j} I_{3 ; j}+c_{2 ; j} I_{2 ; j}+c_{1 ; j} I_{1 ; j}+\mathcal{R}+\mathcal{O}(D-4) .
$$

In Eq. (2.5) the coefficients $c_{N, j}(N=1, \ldots 4)$ are evaluated in $D=4$, i. e. they do not have any dependence on $\epsilon$, and $I_{L ; j}$ stands for an $L$-point one-loop scalar integral of the type $j$. The type $j$ specifies, cryptically, which combinations of the external momenta $p_{i}$ build up the $q_{i}$ that enter the (master) integrals in the right hand side of Eq. (2.5). The existence of this decomposition is one of the most important results for one-loop calculations; its origin relies on simple Lorentz invariance which allows a decomposition of tensor integrals to invariant form factors and on the four-dimensional nature of space time which allows scalar higher point integrals to be reduced to sums of boxes. Thus a scalar pentagon in $D$ dimensions can be written as a sum of the five box diagrams obtainable by removing one propagator if we neglect terms of order $\epsilon[49,50,51]$. The general one loop $N$-point integral in $D=4-2 \epsilon$ dimensions for $N \geq 6$ can be recursively obtained as a linear combination of pentagon integrals $[49,50]$, provided that the external momenta are restricted to four dimensions. This will be discussed in detail in the later sections. The significance of Eq. (2.5) is that once scalar one-loop integrals are tabulated for $N \leq 4$, any one-loop calculation is reduced to the determination of both the coefficients $c_{L ; j}$ and the rational part $\mathcal{R}$. As we shall demonstrate in this review, the reduction coefficients and the rational part, can be obtained by efficient numerical methods based on an algebraic understanding of the structure of the integrand. 


\subsection{One-loop scalar integrals}

We now discuss the set of scalar integrals that appear on the right hand side in Eq. (2.5). These integrals $I_{N}$ - traditionally referred to as tadpoles, bubbles, triangles and boxes - are defined as

$$
\begin{aligned}
& I_{1}\left(m_{1}^{2}\right)=\frac{\mu^{4-D}}{i \pi^{\frac{D}{2}} r_{\Gamma}} \int \frac{d^{D} l}{d_{1}} \\
& I_{2}\left(p_{1}^{2} ; m_{1}^{2}, m_{2}^{2}\right)=\frac{\mu^{4-D}}{i \pi^{\frac{D}{2}} r_{\Gamma}} \int \frac{d^{D} l}{d_{1} d_{2}} \\
& I_{3}\left(p_{1}^{2}, p_{2}^{2}, p_{3}^{2} ; m_{1}^{2}, m_{2}^{2}, m_{3}^{2}\right)=\frac{\mu^{4-D}}{i \pi^{\frac{D}{2}} r_{\Gamma}} \int \frac{d^{D} l}{d_{1} d_{2} d_{3}}, \\
& I_{4}\left(p_{1}^{2}, p_{2}^{2}, p_{3}^{2}, p_{4}^{2} ; s_{12}, s_{23} ; m_{1}^{2}, m_{2}^{2}, m_{3}^{2}, m_{4}^{2}\right)=\frac{\mu^{4-D}}{i \pi^{\frac{D}{2}} r_{\Gamma}} \int \frac{d^{D} l}{d_{1} d_{2} d_{3} d_{4}}
\end{aligned}
$$

where $d_{i}=\left(l+q_{i-1}\right)^{2}-m_{i}^{2}+i \varepsilon, q_{n} \equiv \sum_{i=1}^{n} p_{i}, q_{0}=0, s_{i j}=\left(p_{i}+p_{j}\right)^{2}$ and $r_{\Gamma}=\Gamma^{2}(1-\epsilon) \Gamma(1+\epsilon) / \Gamma(1-2 \epsilon)$.

For real masses $m_{i}$ the results for these four types of integrals are given in Ref. [52]. A further simplification of the general box integral can be found in Ref. [53]. In the case where some of the masses vanish, leading to infrared and collinear divergences, the dimensionally regularized results are given in Ref. [54]. All scalar one-loop integrals for $N \leq 4$ with vanishing or real masses can be obtained from the Fortran 77 program QCDLoop [55]. The extension of these results to cases where some of the internal masses are complex - relevant for calculations with unstable particles - was given in Refs. [56, 57]. As a consequence of these papers, the problem of the analytic calculation of one-loop integrals and of their numerical evaluation can be considered completely solved.

\subsection{One-loop tensor integrals and form factor expansion}

In the calculation of a general one-loop amplitude, individual Feynman diagrams will give rise to tensor integrals containing powers of the loop momentum in the numerator. In a renormalizable theory the number of powers of the loop momentum, $r$ will be limited such that $r \leq N$ where $N$ is the number of external legs. We shall define $r$ to be the rank of the tensor integral. The calculation of these tensor integrals is simple but tedious so it is expedient to reduce the tensor integrals to the scalar integrals presented in the previous Section. This was first proposed by Passarino and Veltman [58], and we give an explanation of their method in this Section. We first define the tensor integrals. For reasons of convenience, we have here adopted an alternative notation, similar to the notation of Passarino and Veltman, for the scalar integrals, $A_{0}, B_{0}, C_{0}, D_{0}$ which correspond to scalar tadpole, bubble, triangle, and box integrals. We write

$$
\begin{gathered}
A_{0}\left(m_{1}\right)=\frac{1}{i \pi^{D / 2}} \int d^{D} l \frac{1}{d_{1}} \\
B_{0} ; B^{\mu} ; B^{\mu \nu}\left(p_{1}, m_{1}, m_{2}\right)=\frac{1}{i \pi^{D / 2}} \int d^{D} l \frac{1 ; l^{\mu} ; l^{\mu} l^{\nu}}{d_{1} d_{2}},
\end{gathered}
$$




$$
\begin{gathered}
C_{0} ; C^{\mu} ; C^{\mu \nu} ; C^{\mu \nu \alpha}\left(p_{1}, p_{2}, m_{1}, m_{2}, m_{3}\right)=\frac{1}{i \pi^{D / 2}} \int d^{D} l \frac{1 ; l^{\mu} ; l^{\mu} l^{\nu} ; l^{\mu} l^{\nu} l^{\alpha}}{d_{1} d_{2} d_{3}} \\
D_{0} ; D^{\mu} ; D^{\mu \nu} ; D^{\mu \nu \alpha} ; D^{\mu \nu \alpha \beta}\left(p_{1}, p_{2}, p_{3}, m_{1}, m_{2}, m_{3}, m_{4}\right)= \\
\frac{1}{i \pi^{D / 2}} \int d^{D} l \frac{1 ; l^{\mu} ; l^{\mu} l^{\nu} ; l^{\mu} l^{\nu} l^{\alpha} ; l^{\mu} l^{\nu} l^{\alpha} l^{\beta}}{d_{1} d_{2} d_{3} d_{4}}
\end{gathered}
$$

where denominators are given by $d_{i}=\left(l+\sum_{k=1}^{i-1} p_{k}\right)^{2}-m_{i}^{2}, i=1, . ., 4$. We give a complete description of the Passarino-Veltman reduction in Appendix A. In the current section we shall present the details of the reduction of rankone and rank-two tensor triangle functions to scalar integrals. This will be sufficient to illustrate the pattern of the reduction and to make clear what are the potential shortcomings of the method. We note that the rank three tensor triangle integrals, which also occur in a renormalizable theory, are treated in Appendix A. As a consequence of Lorentz invariance, we may write

$$
\begin{aligned}
C^{\mu} & =p_{1}^{\mu} C_{1}+p_{2}^{\mu} C_{2}, \\
C^{\mu \nu} & =g^{\mu \nu} C_{00}+\sum_{i, j=1}^{2} p_{i}^{\mu} p_{j}^{\nu} C_{i j}, \quad \text { where } C_{21}=C_{12} .
\end{aligned}
$$

We shall refer to the coefficients $C_{i}, C_{00}, C_{i j}, i, j=1,2$ as form factors. The dependence of these form factors on the Lorentz invariants of the problem, $p_{1}^{2}, p_{2}^{2},\left(p_{1}+p_{2}\right)^{2}, m_{1}^{2}, m_{2}^{2}, m_{3}^{2}$ has been suppressed. We contract both sides of Eq. (2.11) with $p_{1}$ and $p_{2}$. In the numerator of the left-hand side we obtain the following dot products which may be expressed in terms of the denominators,

$$
\begin{aligned}
& l \cdot p_{1}=\frac{1}{2}\left(f_{1}+d_{2}-d_{1}\right), \quad f_{1}=m_{2}^{2}-m_{1}^{2}-p_{1}^{2}, \\
& l \cdot p_{2}=\frac{1}{2}\left(f_{2}+d_{3}-d_{2}\right), \quad f_{2}=m_{3}^{2}-m_{2}^{2}-p_{2}^{2}-2 p_{1} \cdot p_{2} .
\end{aligned}
$$

We use Eqs. $(2.11,2.13,2.14)$ and obtain a system of equations for the coefficients $C_{1}, C_{2}$. It reads

$$
G_{2}\left(\begin{array}{c}
C_{1} \\
C_{2}
\end{array}\right)=\left(\begin{array}{c}
\left\langle l \cdot p_{1}\right\rangle \\
\left\langle l \cdot p_{2}\right\rangle
\end{array}\right)=\left(\begin{array}{c}
R_{1}^{[c]} \\
R_{2}^{[c]}
\end{array}\right),
$$

where $G_{2}$ is the $2 \times 2$ Gram matrix

$$
G_{2}=\left(\begin{array}{cc}
p_{1} \cdot p_{1} & p_{1} \cdot p_{2} \\
p_{1} \cdot p_{2} & p_{2} \cdot p_{2}
\end{array}\right)
$$

and we have introduced the notation

$$
\left\langle l \cdot p_{j}\right\rangle=\int \frac{d^{D} l}{i \pi^{D / 2}} \frac{l \cdot p_{j}}{d_{1} d_{2} d_{3}}, \text { for } j=1,2 .
$$


We use Eqs. $(2.13,2.14)$ to find explicit expressions for the $R_{1,2}^{[c]}$. They are

$$
\begin{aligned}
& R_{1}^{[c]}=\frac{1}{2}\left(f_{1} C_{0}(1,2,3)+B_{0}(1,3)-B_{0}(2,3)\right), \\
& R_{2}^{[c]}=\frac{1}{2}\left(f_{2} C_{0}(1,2,3)+B_{0}(1,2)-B_{0}(1,3)\right) .
\end{aligned}
$$

To express our results in Eq. (2.18) we have introduced a compact notation which labels the form factors by the denominators they contain. Thus, for example, in Eq. (2.18), $B_{0}(2,3)$ is defined as the integral

$$
B_{0}(2,3) \equiv B_{0}\left(p_{2}, m_{2}, m_{3}\right)=\int \frac{d^{D} l}{i \pi^{D / 2}} \frac{1}{\left(l^{2}-m_{2}^{2}\right)\left(\left(l+p_{2}\right)^{2}-m_{3}^{2}\right)} .
$$

Note that in Eq. (2.19) the loop momentum $l$ has been shifted with respect to the defining equation for the triangle integrals because $d_{1}$ has been cancelled. Finally, solving the system of equations (2.15) we obtain,

$$
\left(\begin{array}{l}
C_{1} \\
C_{2}
\end{array}\right)=G_{2}^{-1}\left(\begin{array}{c}
R_{1}^{[c]} \\
R_{2}^{[c]}
\end{array}\right) .
$$

A similar contraction procedure can also be applied to the rank-two tensor triangle integral, Eq. (2.12), or to higher rank tensors as described in Appendix A. For example, contracting Eq. (2.12) with $p_{1}$ and $p_{2}$ we obtain

$$
\begin{aligned}
& p_{1 \mu} C^{\mu \nu}=p_{1}^{\nu}\left(p_{1} \cdot p_{1} C_{11}+p_{1} \cdot p_{2} C_{12}+C_{00}\right)+p_{2}^{\nu}\left(p_{1} \cdot p_{1} C_{12}+p_{1} \cdot p_{2} C_{22}\right), \\
& p_{2 \mu} C^{\mu \nu}=p_{1}^{\nu}\left(p_{1} \cdot p_{2} C_{11}+p_{2} \cdot p_{2} C_{12}\right)+p_{2}^{\nu}\left(p_{1} \cdot p_{2} C_{12}+p_{2} \cdot p_{2} C_{22}+C_{00}\right) .
\end{aligned}
$$

Using Eqs. $(2.13,2.14)$ we can derive the following two equations

$$
G_{2}\left(\begin{array}{c}
C_{11} \\
C_{12}
\end{array}\right)=\left(\begin{array}{c}
R_{1}^{[c 1]} \\
R_{2}^{[c 1]}
\end{array}\right), \quad G_{2}\left(\begin{array}{c}
C_{12} \\
C_{22}
\end{array}\right)=\left(\begin{array}{c}
R_{1}^{[c 2]} \\
R_{2}^{[c 2]}
\end{array}\right),
$$

where

$$
\begin{aligned}
& R_{1}^{[c 1]}=\frac{1}{2}\left(f_{1} C_{1}(1,2,3)+B_{1}(1,3)+B_{0}(2,3)-2 C_{00}(1,2,3)\right), \\
& R_{2}^{[c 1]}=\frac{1}{2}\left(f_{2} C_{1}(1,2,3)+B_{1}(1,2)-B_{1}(1,3)\right),
\end{aligned}
$$

and

$$
\begin{aligned}
& R_{1}^{[c 2]}=\frac{1}{2}\left(f_{1} C_{2}(1,2,3)+B_{1}(1,3)-B_{1}(2,3)\right), \\
& R_{2}^{[c 2]}=\frac{1}{2}\left(f_{2} C_{2}(1,2,3)-B_{1}(1,3)-2 C_{00}(1,2,3)\right) .
\end{aligned}
$$

In this way we obtain a ladder of relations which allow us to express a rank $r$ triangle form-factors in terms of rank $r-1$ triangle form-factors and sums 


\begin{tabular}{|lll|}
\hline$D_{i j k l}$ & $\rightarrow$ & $D_{00 i j}, D_{i j k}, C_{i j k}, C_{i j}, C_{i}, C_{0}$ \\
$D_{00 i j}$ & $\rightarrow$ & $D_{i j k}, D_{i j}, C_{i j}, C_{i}$ \\
$D_{0000}$ & $\rightarrow$ & $D_{00 i}, D_{00}, C_{00}$ \\
$D_{i j k}$ & $\rightarrow$ & $D_{00 i}, D_{i j}, C_{i j}, C_{i}$ \\
$D_{00 i}$ & $\rightarrow$ & $D_{i j}, D_{i}, C_{i}, C_{0}$ \\
$D_{i j}$ & $\rightarrow$ & $D_{00}, D_{i}, C_{i}, C_{0}$ \\
$D_{00}$ & $\rightarrow$ & $D_{i}, D_{0}, C_{0}$ \\
$D_{i}$ & $\rightarrow$ & $D_{0}, C_{0}$ \\
\hline$C_{i j k}$ & $\rightarrow$ & $C_{00 i}, C_{i j}, B_{i j}, B_{i}$ \\
$C_{00 i}$ & $\rightarrow$ & $C_{i i}, C_{i}, B_{i}, B_{0}$ \\
$C_{i j}$ & $\rightarrow$ & $C_{00}, C_{i}, B_{i}, B_{0}$ \\
$C_{00}$ & $\rightarrow$ & $C_{i}, C_{0}, B_{0}$ \\
$C_{i}$ & $\rightarrow$ & $C_{0}, B_{0}$ \\
\hline$B_{i i}$ & $\rightarrow$ & $B_{00}, B_{i}, A_{0}$ \\
$B_{00}$ & $\rightarrow$ & $B_{i}, B_{0}, A_{0}$ \\
$B_{i}$ & $\rightarrow$ & $B_{0}, A_{0}$ \\
\hline
\end{tabular}

Table 2.1: Reduction chains for Passarino-Veltman procedure, see Appendix A for a definition of all coefficients.

of bubble form-factors, with rank $r-1$ or less. Thus, as a general rule, the Passarino-Veltman procedure relates rank $r$ form-factors of a Feynman integral with $N$ denominators to rank $r-1$ form-factors of Feynman integrals with $N-1$ denominators plus other terms which are less ultraviolet singular. The full pattern of reduction to scalar integrals is given in Table 2.1.

The exception to this rule is the $C_{00}$ term that we treat below. We also note that, since the external vectors are purely four-dimensional, the contraction procedure that we just described does not introduce an explicit dependence on the dimensionality of space-time in the reduction equations.

To find the $C_{00}$ coefficient, we note that a further relation can be obtained by contracting the rank-two tensor integral in Eq. (2.12) with the metric tensor $g^{\mu \nu}$. We find

$$
\left\langle l^{2}-m_{1}^{2}\right\rangle=D C_{00}+R_{1}^{[c 1]}+R_{2}^{[c 2]}-m_{1}^{2} C_{0} .
$$

Inserting the explicit forms from Eqs. $(2.23,2.24)$ we find,

$$
\begin{aligned}
C_{00}(1,2,3) & =\frac{1}{2(D-2)}\left(2 m_{1}^{2} C_{0}(1,2,3)-f_{2} C_{2}(1,2,3)-f_{1} C_{1}(1,2,3)\right. \\
& \left.+B_{0}(2,3)\right) .
\end{aligned}
$$

Therefore, we see that a pattern of reduction appears

$$
\begin{aligned}
C_{i j} & \rightarrow C_{00}, C_{i}, B_{i},\left(B_{0}\right), \\
C_{00} & \rightarrow C_{i},\left(C_{0}, B_{0}\right) \\
C_{i} & \rightarrow\left(C_{0}, B_{0}\right) .
\end{aligned}
$$


The scalar integrals in the reduction path are shown in brackets.

A simple generalization of the above procedure accomplishes the reduction of all tensor integrals to scalar integrals. A reduction of six- and higher-point functions requires additional input, since the external momenta are not linearly independent, but the basic principles remain intact. It appears therefore, that the reduction procedure is a well-established technique that can be applied to any process of interest in a straightforward way.

However, there are three primary reasons for why it is non-trivial to perform such a reduction in practice for complicated collider processes. First, the number of Feynman diagrams grows dramatically with the number of external particles. For the LHC processes of interest, the number of diagrams can easily reach a few thousand. Second, the number of terms generated during the reduction of tensor integrals grows rapidly with the number of external particles and with the rank of the integral. Third, in cases with degenerate kinematics, the traditional reduction procedure may lead to numerical instabilities which we consider in Section 2.4 .

\subsection{Singular regions}

As an example, we consider the reduction of the rank one triangle. The form factors for this integral can be found by solving Eq. (2.15). We obtain

$$
\left(\begin{array}{c}
C_{1} \\
C_{2}
\end{array}\right)=G_{2}^{-1}\left(\begin{array}{c}
\left\langle l \cdot p_{1}\right\rangle \\
\left\langle l \cdot p_{2}\right\rangle
\end{array}\right)
$$

where the inverse of the Gram matrix is given by

$$
G_{2}^{-1}=\frac{\left(\begin{array}{cc}
p_{2} \cdot p_{2} & -p_{1} \cdot p_{2} \\
-p_{1} \cdot p_{2} & p_{1} \cdot p_{1}
\end{array}\right)}{\Delta_{2}\left(p_{1}, p_{2}\right)},
$$

and

$$
\Delta_{2}\left(p_{1}, p_{2}\right)=\operatorname{det}\left[G_{2}\right]=p_{1}^{2} p_{2}^{2}-\left(p_{1} \cdot p_{2}\right)^{2},
$$

is the determinant of the Gram matrix, the so-called Gram determinant.

We now investigate the solution Eq. (2.28) in the limit $p_{1} \| p_{2}$ with $p_{1}^{2} \neq 0$. In this limit, the Gram determinant $\Delta_{2}$ vanishes, so that the inverse matrix $G_{2}^{-1}$ needed for the construction of the solution Eq. (2.28) cannot be obtained. On the other hand, the original integral, $C^{\mu}$, Eq. (2.9), is well-defined in that limit. Therefore, the problem appears because we attempt to treat the two momenta $p_{1}^{\mu}$ and $p_{2}^{\mu}$ as independent in the form factor expansion Eq. (2.11) even in a situation when they are linearly dependent.

It is easy to remedy this situation, at least in this simple case, by using linearly-independent momenta. To this end, we introduce

$$
\tilde{p}_{2}^{\mu}=p_{2}^{\mu}-\frac{p_{1} \cdot p_{2}}{p_{1}^{2}} p_{1}^{\mu},
$$

and write

$$
C^{\mu}=p_{1}^{\mu} \tilde{C}_{1}+\tilde{p}_{2}^{\mu} \tilde{C}_{2}
$$


instead of decomposition shown in Eq. (2.11).

Since $p_{1} \cdot \tilde{p}_{2}=0$, it is easy to solve for $\tilde{C}_{1}$ and $\tilde{C}_{2}$. By contracting the integral with $p_{1,2}$ we obtain the set of equations

$$
\left\langle l \cdot p_{1}\right\rangle=p_{1}^{2} \tilde{C}_{1}, \quad\left\langle l \cdot p_{2}\right\rangle=p_{2} \cdot p_{1} \tilde{C}_{1}+\frac{\Delta_{2}\left(p_{1}, p_{2}\right)}{p_{1}^{2}} \tilde{C}_{2},
$$

and find

$$
\tilde{C}_{1}=\frac{\left\langle l \cdot p_{1}\right\rangle}{p_{1}^{2}}, \quad \tilde{C}_{2}=\frac{p_{1}^{2}\left\langle l \cdot \tilde{p}_{2}\right\rangle}{\Delta_{2}\left(p_{1}, p_{2}\right)}
$$

We can now analyze the limit $p_{1} \| p_{2}$. We write $p_{2}^{\mu}=\kappa p_{1}^{\mu}+\delta n^{\mu}, n \cdot p_{1}=0$, $n^{2}=1, \delta \ll 1$. It follows that $\Delta\left(p_{1}, p_{2}\right)=\delta^{2} p_{1}^{2}$ and $\tilde{p}_{2}=\delta n^{\mu}$. It is easy to see from Eq. (2.34) that $p_{1}^{\mu} \tilde{C}_{1}$ is finite in the limit $p_{1} \| p_{2}$ and, because the integral $C^{\mu}$ is also finite in that limit, $\tilde{p}_{2}^{\mu} \tilde{C}_{2}$ must be finite as well. However this finiteness occurs because there is a cancellation between numerator and denominator in the expression for $C^{\mu}$ in Eq. (2.32). In detail, $\tilde{p}_{2}^{\mu}$ is $O(\delta)$ and $\tilde{C}_{2}$ is $O(1 / \delta)$. These features - and the ensuing cancellations - become obscured if $\tilde{C}_{2}$ is rewritten in terms of master integrals. In this case the finiteness of $\tilde{C}_{2}$ is achieved through the cancellation of a number of $\mathcal{O}\left(\delta^{-2}\right)$ terms, including non-trivial relations between three- and two- point integrals which, in the limit $p_{2} \rightarrow \kappa p_{1}$, become linearly dependent.

The situation described here generalizes to more complicated cases: a bruteforce application of the Passarino-Veltman reduction procedure can lead to numerical instabilities due to the vanishing of Gram determinant at so called "exceptional points", despite the fact that no singularity is present in the original integral. We will discuss in Sections 2.5 and 10 how to rescue these exceptional points.

\subsection{Advanced diagrammatic methods}

The computational algorithms for tree and one-loop calculations that employ Feynman diagrams are suitable for automation. There are public codes for generating Feynman diagrams [59, 60] which, in conjunction with algebraic manipulation codes such as Form, Maple and Mathematica, allow to automatically generate Fortran or $\mathrm{C}$ computer codes that numerically compute scattering amplitudes or cross-sections. This type of approach is used in many applications to calculate physical observables at leading and next-to-leading order accuracy.

Unfortunately, computational approaches based on Feynman diagrams experience worse than factorial scaling with the number of the external particles. As the number of external particles grows, high-rank tensor integrals appear in virtual diagrams. The Passarino-Veltman reduction generates a multitude of terms and the number of these terms grows faster than exponentially with the rank of the tensor. Furthermore, as we already mentioned, in the numerical evaluation of one-loop amplitudes one needs to address the issue of numerical stability related to the vanishing of Gram determinants. 
The main subject of this review is to describe an alternative to Feynmandiagrammatic methods. Still, we emphasize that the question of when the practical limit on Feynman-diagrammatic one-loop calculations is reached remains open. For a long time it was believed that it was extremely hard, if not altogether impossible, for Feynman-diagrammatic computations to pass the $2 \rightarrow 3$ threshold and deliver physical results for $2 \rightarrow 4$ processes. Yet, in 2009 this threshold was successfully passed: as the result of additional technical improvements, techniques based on Feynman diagrams were successfully used to describe $p p \rightarrow t \bar{t} b \bar{b}$ and $p p \rightarrow W^{+} W^{-} b \bar{b}$ processes at next-to-leading in perturbative QCD $[2,12]$. Similar ideas are implemented in the GOLEM program $[61,62]$ and first results on the next-to-leading order computation of processes with six external particles have been presented by the GOLEM collaboration.

Taking as an example the process $p p \rightarrow t \bar{t} b \bar{b}$, we note that at next-to-leading order in perturbative QCD, it involves two partonic channels $q \bar{q} \rightarrow b \bar{b} t \bar{t}$ and $g g \rightarrow b \bar{b} t \bar{t}$. There are respectively 188 and 1003 loop diagrams that contribute to the one-loop amplitudes for the two channels [2]. The computational cost of having to deal with a large number of Feynman diagrams is compensated by a careful organization of the computation. The key idea is to decouple, to the extent possible, the reduction of tensor loop integrals and other operations such as multiplication of gamma matrices and spinors, summations of colors and helicities, etc. from each other. This is achieved by paying careful attention to the following issues [2].

First, the interference of the leading (LO) and next-to-leading order (NLO) matrix elements is decomposed into the individual contributions of loop diagrams $\Gamma$. The sum over helicities and colors are performed for each loop diagram separately

$$
\sum_{\text {col }} \sum_{\text {hel }} \mathcal{M}^{(\mathrm{NLO})}\left(\mathcal{M}^{(\mathrm{LO})}\right)^{*}=\sum_{\Gamma}\left[\sum_{\text {col }} \sum_{\text {hel }} \mathcal{M}^{(\Gamma)}\left(\mathcal{M}^{(\mathrm{LO})}\right)^{*}\right] .
$$

Second, each individual loop diagram has a color factor that can be expanded in a compact color basis. The leading order amplitude is treated as vector in this color basis and it is contracted against the color factor of the loop diagram.

Third, similar to the treatment of color, the spin-dependent parts of all diagrams can be expanded in a compact spin basis for a given channel. For example, a representative term in the spin basis needed to describe the fourquark, two-gluon channel has the form

$$
\begin{aligned}
\hat{S}=Q^{\mu_{1} \mu_{2} \rho_{1} \ldots \rho_{l}} \epsilon_{\mu_{1}}\left(p_{1}\right) \epsilon_{\mu_{2}}\left(p_{2}\right) & {\left[\bar{v}\left(p_{3}\right) \gamma_{\rho_{1}} \ldots \gamma_{\rho_{m}} u\left(p_{4}\right)\right] } \\
& \times\left[v\left(\bar{p}_{5}\right) \gamma_{\rho_{m+1}} \ldots \gamma_{\rho_{l}} u\left(p_{6}\right)\right],
\end{aligned}
$$

where $Q^{\mu_{1} \mu_{2} \rho_{1} \ldots \rho_{l}}$ is a tensor of the appropriate rank that is composed of metric tensors and external momenta. The use of a compact spin basis enables very efficient helicity summations.

Fourth, clever techniques are used for tensor integral reductions. Those techniques minimize difficulties related to inverse Gram determinants and employ a cache system to recycle tensor integrals with common sub-topologies and 
avoid the computation of relevant scalar integrals more than once. For tensor $N$-point integrals with $N \geq 5$, a procedure can be used that reduces the number of propagators and the rank of the corresponding integral at the same time [63]. Such a procedure does not introduce inverse Gram determinants. In the case of three- and four-point functions, where the reduction does introduce small Gram determinants, an expansion procedure around the limit of vanishing Gram determinants and other relevant kinematical structures is applied [63, 64].

Fifth, careful identification of terms that need to be treated in $D$ dimensions and terms that can be treated in four dimensions, plays an important role in achieving high computational efficiency. Finally, it was observed [2] that it is possible to give up on the optimization of the spin basis. Indeed, in earlier work it was considered crucial that a minimal, absolutely independent number of Lorentz structures was used in the parametrization of the one-loop amplitude. Instead, it was found in Ref. [2] that one does not lose computational efficiency by only employing generic four-dimensional identities to reduce the number of independent terms in the spin basis. This feature is important since it minimizes the amount of human intervention in the simplification of spinor chains.

\section{Van Neerven - Vermaseren basis}

\subsection{The Van Neerven - Vermaseren basis}

On-shell scattering amplitudes in gauge field theories are gauge-invariant. A practical version of this statement is that an on-shell scattering amplitude must vanish, if evaluated replacing the polarization vector of a particular massless gauge boson by its four-momentum, provided all the other gauge bosons have physical polarizations. This provides both a constraint on the form of the amplitude and a powerful check on the computation. However, it is well-known that in complicated cases involving higher-point scattering amplitudes, the analytic demonstration of this cancellation is non-trivial. One reason why such complications arise is the four-dimensional nature of space-time, since it implies that for high-point amplitudes the external momenta are not linearly independent. In four dimensions, the "dimensionality constraint" can be stated in the form of the Schouten identity

$$
l^{\lambda} \epsilon^{\mu_{1} \mu_{2} \mu_{3} \mu_{4}}=l^{\mu_{1}} \epsilon^{\lambda \mu_{2} \mu_{3} \mu_{4}}+l^{\mu_{2}} \epsilon^{\mu_{1} \lambda \mu_{3} \mu_{4}}+l^{\mu_{3}} \epsilon^{\mu_{1} \mu_{2} \lambda \mu_{4}}+l^{\mu_{4}} \epsilon^{\mu_{1} \mu_{2} \mu_{3} \lambda},
$$

which follows from the vanishing of the totally antisymmetric rank-five tensor in four dimensions. Since these constraints are not implemented in the PassarinoVeltman procedure, it is usually not easy to demonstrate gauge cancellations in that framework.

The Schouten identities also provide us with a way to introduce a particular reference frame that, as we will see, is very useful for reducing tensor one-loop integrals to scalar integrals. To motivate this choice, consider a two-dimensional vector space spanned by two non-orthogonal two-dimensional vectors $q_{1}^{\mu_{1}}, q_{2}^{\mu_{2}}$. Any two-dimensional vector from that vector space can be written as a linear combination of these vectors $l^{\alpha}=c_{1} q_{1}^{\alpha}+c_{2} q_{2}^{\alpha}$. However, since $q_{1}, q_{2}$ are not 
orthogonal, $c_{i} \neq l \cdot q_{i}$. A standard way to introduce the orthonormal basis is the Gram-Schmidt orthogonalization procedure, but we will not pursue it here. Instead we will choose a convenient basis starting from Schouten identity in two dimensions

$$
l^{\lambda} \epsilon^{\mu_{1} \mu_{2}}=l^{\mu_{1}} \epsilon^{\lambda \mu_{2}}+l^{\mu_{2}} \epsilon^{\mu_{1} \lambda} .
$$

Contracting both sides of this equation with $q_{1}^{\mu_{1}} q_{2}^{\mu_{2}}$ we obtain

$$
l^{\lambda} \epsilon^{q_{1} q_{2}}=\left(l \cdot q_{1}\right) \epsilon^{\lambda q_{2}}+\left(l \cdot q_{2}\right) \epsilon^{q_{1} \lambda}
$$

where $e^{\lambda q_{2}}=\epsilon^{\lambda \mu_{2}} q_{2, \mu_{2}}$ and $\epsilon^{q_{1} q_{2}}=\epsilon^{\mu_{1} \mu_{2}} q_{1, \mu_{1}} q_{2, \mu_{2}}$. We divide both sides by $\epsilon^{q_{1} q_{2}}$, introduce vectors ${ }^{2}$

$$
v_{1}^{\lambda}=\frac{\epsilon^{\lambda q_{2}}}{\epsilon^{q_{1} q_{2}}}, \quad v_{2}^{\lambda}=\frac{\epsilon^{q_{1} \lambda}}{\epsilon^{q_{1} q_{2}}}, \quad v_{i} \cdot q_{j}=\delta_{i j}
$$

and write

$$
l^{\lambda}=\left(l \cdot q_{1}\right) v_{1}^{\lambda}+\left(l \cdot q_{2}\right) v_{2}^{\lambda} .
$$

The vector sets $\left\{v_{i}\right\}$ and $\left\{q_{j}\right\}$ are orthogonal, but vectors $v_{i}$ are not orthonormal, $v_{i} \cdot v_{j} \neq \delta_{i j}$. Nevertheless, they are useful because they define a coordinate system where the $v_{i}$-coordinate of an arbitrary vector $l$ is the projection of this vector on the vector $q_{i}$. If we identify $l+q_{i}$ with momenta that appear in propagators of one-loop diagrams (see Fig. 3.1), the scalar product $l \cdot q_{i}$ can be replaced by differences of denominators

$$
l \cdot q_{i}=\frac{1}{2}\left[\left(\left(l+q_{i}\right)^{2}-m_{i}^{2}\right)-\left(l^{2}-m_{0}^{2}\right)+m_{i}^{2}-m_{0}^{2}-q_{i}^{2}\right] .
$$

This strategy has already been used in the Passarino-Veltman reduction, c.f. Eqs. $(2.13,2.14)$, and as we explain later in the review, this feature will be used to develop a systematic procedure to determine the parametric form of one-loop integrands.

The two-dimensional example, however, is not sufficient since we have to deal with higher-dimensional vector spaces. We therefore need to extend the considerations described above. To this end, we write

$$
v_{1}^{\mu}=\frac{\epsilon_{q_{1} q_{2}} \epsilon^{\mu q_{2}}}{\epsilon_{q_{1} q_{2}} \epsilon^{q_{1} q_{2}}}, \quad v_{2}^{\mu}=\frac{\epsilon_{q_{1} q_{2}} \epsilon^{q_{1} \mu}}{\epsilon_{q_{1} q_{2}} \epsilon^{q_{1} q_{2}}},
$$

and use

$$
\epsilon^{\mu_{1} \mu_{2}} \epsilon_{\nu_{1} \nu_{2}}=\delta_{\nu_{1}}^{\mu_{1}} \delta_{\nu_{2}}^{\mu_{2}}-\delta_{\nu_{2}}^{\mu_{1}} \delta_{\nu_{1}}^{\mu_{2}}=\operatorname{det}\left|\delta_{\nu}^{\mu}\right| \equiv \delta_{\nu_{1} \nu_{2}}^{\mu_{1} \mu_{2}}
$$

to write vectors $v_{1,2}$ using the basis of generalized Kronecker delta-symbols

$$
v_{1}^{\mu}=\frac{\delta_{q_{1} q_{2}}^{\mu q_{2}}}{\Delta_{2}}, \quad v_{2}^{\mu}=\frac{\delta_{q_{1} q_{2}}^{q_{1} \mu}}{\Delta_{2}}, \quad \Delta_{2}=\delta_{q_{1} q_{2}}^{q_{1} q_{2}}=q_{1}^{2} q_{2}^{2}-\left(q_{1} \cdot q_{2}\right)^{2} .
$$

\footnotetext{
${ }^{2}$ The case $\epsilon^{q_{1} q_{2}} \rightarrow 0$ requires special care, see for instance the discussion in Sect. 2.4.
} 
We recognize that, in contrast to Levi-Civita tensors, the generalized Kronecker deltas can be introduced for vector spaces of arbitrary dimensions, allowing us to define the basis of dual vectors $v_{i}$ that can be used in four-dimensional calculations. Such basis is called the van Neerven - Vermaseren basis [50] .

The van Neerven - Vermaseren basis has proved to be very useful to understand a number of important results concerning the reduction of tensor integrals and the applicability of the generalized unitarity. First, in four dimensions, simple algorithms were derived for the reduction of tensor integrals to the linear combination of box, triangle, bubble and tadpole scalar integrals. The number of terms generated in this process is smaller than in the standard PassarinoVeltman reduction procedure. Some illustrative results are presented in Section 4. Second, using the van Neerven - Vermaseren basis, it is straightforward to show that in four dimensions the scalar five-point Feynman integral is given by a linear combination of scalar box integrals $[49,50]$. Third, using the van Neerven - Vermaseren basis it is easy to understand that in four dimensions the integrand of any one-loop Feynman diagram in any renormalizable theory is given by a linear combination of rational functions containing products of four, three, two or one Feynman denominators and with the numerators of a very restrictive form, see Section 5. Finally, employing the van Neerven - Vermaseren decomposition, it is straightforward to find the loop momenta that satisfy quadruple, triple-, double-, and single-cut on-shell conditions, see Section 5. These features of the van Neerven - Vermaseren basis make it important for the construction of the generalized $D$-dimensional unitarity technique. We therefore devote the following subsection to a detailed explanation of the van Neerven - Vermaseren basis.

\subsection{Physical and transverse space}

We consider a $N$-particle scattering amplitude in a renormalizable quantum field theory in $D$-dimensional space-time. Such an amplitude can be computed from the relevant Feynman diagrams, each given by an integral over the loop momentum $l$ of an integrand function. We study one of these Feynman diagrams and imagine that it has $R$ loop-momentum-dependent propagators, see Fig. 3.1. The integrand $\mathcal{I}_{N}$ is a rational function of the loop momentum $l$ given by the product of $R, l$-dependent scalar inverse propagators $d_{i}$ and a polynomial in $l$ of rank $r_{l} \leq R$.

$$
\mathcal{I}_{N}\left(p_{1}, p_{2}, \ldots, p_{N} \mid l\right)=\frac{\mathcal{N}_{\mathcal{I}}\left(p_{1}, p_{2}, \ldots, p_{N} ; l\right)}{d_{1} d_{2} \cdots d_{R}} .
$$

The amplitude has a set of $R$ inflow momenta, $k_{1}, \ldots, k_{R}$. The inflow momenta are either equal to the external momenta $p_{i}$, or are given by their linear combinations

$$
d_{i}=\left(l+q_{i}\right)^{2}-m_{i}^{2}, \quad k_{i}=q_{i}-q_{i-1}, \quad k_{i}=\sum_{j=1}^{N} \alpha_{i j} p_{j}, \quad \sum_{i=1}^{R} k_{i}=0,
$$




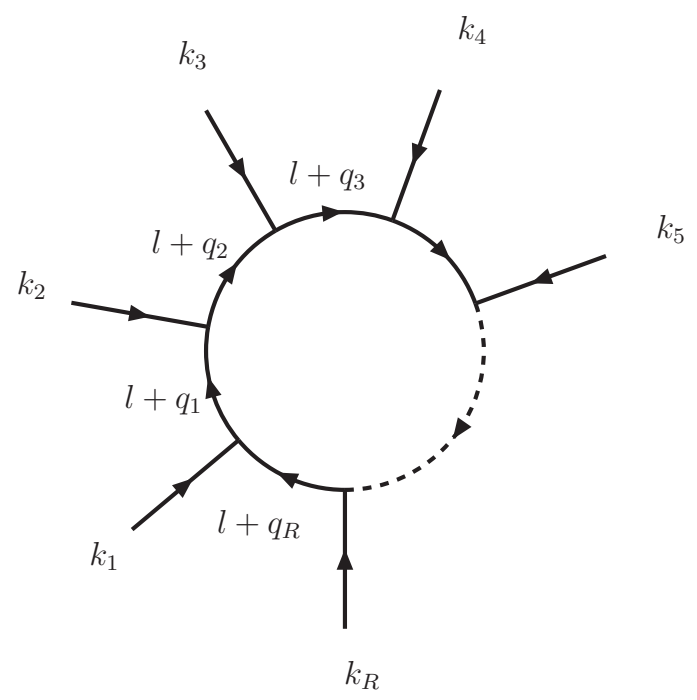

Figure 3.1: Generic diagram with $R$ external momenta at one-loop.

where $\alpha_{i j}=0,1$ are diagram-specific numbers. Sometimes we refer to the $q_{i}$ vectors as the "propagator momenta". We call the vector space spanned by the inflow momenta the physical space. We emphasize that the dimensionality $D_{P}$ of the physical space changes from diagram to diagram. Accounting for momentum conservation $\sum_{i=1}^{R} k_{i}=0$, we obtain

$$
D_{P}=\min (D, R-1),
$$

which implies that for $R \leq D$, the dimensionality of the physical space is smaller than the dimensionality of space-time. The authors of Ref. [50] advocate the use of a non-orthogonal coordinate system in the physical space. This coordinate system is dual to the non-orthogonal coordinate system of the inflow momenta. By contrast, in the $D_{T}$-dimensional transverse space we can use a standard ortho-normal coordinate system. The dimensionalities of various spaces satisfy obvious constraints

$$
D=D_{P}+D_{T}, \quad D_{P}=\min (D, R-1), \quad D_{T}=\max (0, D-R+1) .
$$

If $R>D$, the transverse space is zero-dimensional.

To define the van Neerven - Vermaseren basis we introduce the generalized 
Kronecker symbol [65] ${ }^{3}$

$$
\delta_{\nu_{1} \nu_{2} \cdots \nu_{R}}^{\mu_{1} \mu_{2} \cdots \mu_{R}}=\left|\begin{array}{cccc}
\delta_{\nu_{1}}^{\mu_{1}} & \delta_{\nu_{2}}^{\mu_{1}} & \ldots & \delta_{\nu_{R}}^{\mu_{1}} \\
\delta_{\nu_{1}}^{\mu_{2}} & \delta_{\nu_{2}}^{\mu_{2}} & \ldots & \delta_{\nu_{R}}^{\mu_{2}} \\
\vdots & \vdots & & \vdots \\
\delta_{\nu_{1}}^{\mu_{R}} & \delta_{\nu_{2}}^{\mu_{R}} & \ldots & \delta_{\nu_{R}}^{\mu_{R}}
\end{array}\right|
$$

a compact notation for the Kronecker symbol contracted with momenta,

$$
\delta_{\nu_{1} k \cdots \nu_{R}}^{k \mu_{2} \cdots \mu_{R}} \equiv \delta_{\nu_{1} \nu_{2} \cdots \nu_{R}}^{\mu_{1} \mu_{2} \cdots \mu_{R}} k_{\mu_{1}} k^{\nu_{2}},
$$

and the $R$-particle Gram determinant

$$
\Delta\left(k_{1}, k_{2}, \cdots, k_{R}\right)=\delta_{k_{1} k_{2} \cdots k_{R}}^{k_{1} k_{2} \cdots k_{R}} .
$$

Note that for $R \geq D+1$ the generalized Kronecker delta vanishes. For the special case $D=R$ the Kronecker delta factorizes into the product of two LeviCivita tensors $\delta_{\nu_{1} \nu_{2} \cdots \nu_{R}}^{\mu_{1} \mu_{2} \cdots \mu_{R}}=\varepsilon^{\mu_{1} \mu_{2} \cdots \mu_{R}} \varepsilon_{\nu_{1} \nu_{2} \cdots \nu_{R}}$. The generalized Kronecker-deltas are determinants of $R$-dimensional matrices. For example for $R=2$ and $R=3$ we have the expressions

$$
\begin{aligned}
\delta_{k_{1} \mu}^{k_{1} k_{2}} & =k_{1} \cdot k_{1} \delta_{\mu}^{k_{2}}-k_{1 \mu} \delta_{k_{1}}^{k_{2}},=k_{1} \cdot k_{1} k_{2 \mu}-k_{2} \cdot k_{1} k_{1 \mu} \\
\delta_{k_{1} k_{2} k_{3}}^{k_{1} k_{2} k_{3}} & =k_{1} \cdot k_{1} \delta_{k_{2} k_{3}}^{k_{2} k_{3}}-k_{1} \cdot k_{2} \delta_{k_{1} k_{3}}^{k_{2} k_{3}}+k_{1} \cdot k_{3} \delta_{k_{1} k_{2}}^{k_{2} k_{3}} \\
& =k_{1} \cdot k_{1}\left(k_{2} \cdot k_{2} k_{3} \cdot k_{3}-k_{2} \cdot k_{3} k_{3} \cdot k_{2}\right) \\
& -k_{1} \cdot k_{2}\left(k_{2} \cdot k_{1} k_{3} \cdot k_{3}-k_{2} \cdot k_{3} k_{3} \cdot k_{1}\right) \\
& +k_{1} \cdot k_{3}\left(k_{2} \cdot k_{1} k_{3} \cdot k_{2}-k_{2} \cdot k_{2} k_{3} \cdot k_{1}\right) .
\end{aligned}
$$

We can use the Kronecker $\delta$-symbols to construct the van Neerven - Vermaseren basis vectors for the physical space $D_{P}$

$$
v_{i}^{\mu}\left(k_{1}, \ldots, k_{D_{P}}\right) \equiv \frac{\delta_{k_{1} \ldots k_{i-1} k_{i} k_{i+1} \ldots k_{D_{P}}}^{k_{1} \ldots k_{i-1} \mu k_{i+1} \ldots k_{D_{P}}}}{\Delta\left(k_{1}, \ldots, k_{D_{P}}\right)},
$$

The basis vectors satisfy orthogonality and normalization properties

$$
v_{i} \cdot k_{j}=\delta_{i j}, \quad \text { for } \quad j \leq D_{P} .
$$

When $R \leq D$ it is convenient to define also the projection operator onto the transverse space

$$
w_{\mu}^{\nu}\left(k_{1}, \ldots, k_{R-1}\right) \equiv \frac{\delta_{k_{1} \ldots k_{R-1} \mu}^{k_{1} \ldots k_{R-1} \nu}}{\Delta\left(k_{1}, \ldots, k_{R-1}\right)}
$$

\footnotetext{
${ }^{3}$ This notation is closely related to the asymmetric Gram determinant notation of Ref. [66],$$
G\left(\begin{array}{ccc}
k_{1} & \cdots & k_{R} \\
q_{1} & \cdots & q_{R}
\end{array}\right)=\delta_{q_{1} q_{2} \cdots q_{R}}^{k_{1} k_{2} \cdots k_{R}} .
$$ 
It fulfills the properties of a projection operator

$$
w_{\mu}{ }^{\mu}=D_{T}=D+1-R, k_{i}^{\mu} w_{\mu \nu}=0, w_{\alpha}^{\mu} w^{\alpha \nu}=w^{\mu \nu} .
$$

We denote the orthonormal unit vectors of the transverse space by $n_{r}^{\mu}, r=$ $1 \ldots D_{T}$. They satisfy the standard orthogonality and normalization requirements

$$
n_{r} \cdot n_{s}=\delta_{r s}, k_{i} \cdot n_{r}=0, v_{i} \cdot n_{r}=0, \quad w^{\mu \nu}=\sum_{r=1}^{D_{T}} n_{r}^{\mu} n_{r}^{\nu},
$$

where $i=1 \ldots D_{P}, r, s=1 \ldots D_{T}$ and $w^{\mu \nu}$ is the metric tensor of the transverse subspace. The tensor decomposition of the full metric tensor is given by the expression

$$
g^{\mu \nu}=\sum_{i=1}^{D_{P}} k_{i}^{\mu} v_{i}^{\nu}+w^{\mu \nu}=\sum_{i=1}^{D_{P}} k_{i}^{\mu} v_{i}^{\nu}+\sum_{i=1}^{D_{T}} n_{i}^{\mu} n_{i}^{\nu} .
$$

Note that the right hand side of this equation is, actually, a symmetric tensor since, by explicitly writing the generalized Kronecker delta-function using $k_{i}$ vectors, one can show that the following equation holds

$$
\sum_{i=1}^{D_{P}} k_{i}^{\mu} v_{i}^{\nu}=\sum_{i=1}^{D_{P}} k_{i}^{\nu} v_{i}^{\mu} .
$$

For the case $D=R$, the transverse space is one-dimensional and the unit vector $n_{1}$ is proportional to a Levi-Civita tensor. For the cases $R<D$ it is a simple task to construct explicitly the $n_{r}^{\mu}$ basis vectors that fulfill the requirements given in Eq. (3.22). As an example, if $D=4$ and $R=4$, we get

$$
\begin{aligned}
& v_{1}^{\mu}\left(k_{1}, k_{2}, k_{3}\right)=\frac{\delta_{k_{1} k_{2} k_{3}}^{\mu k_{2} k_{3}}}{\Delta\left(k_{1}, k_{2}, k_{3}\right)}, v_{2}^{\mu}\left(k_{1}, k_{2}, k_{3}\right)=\frac{\delta_{k_{1} k_{2} k_{3}}^{k_{1} \mu k_{3}}}{\Delta\left(k_{1}, k_{2}, k_{3}\right)}, \\
& v_{3}^{\mu}\left(k_{1}, k_{2}, k_{3}\right)=\frac{\delta_{k_{1} k_{2} k_{3}}^{k_{1} k_{2} \mu}}{\Delta\left(k_{1}, k_{2}, k_{3}\right)} ; \\
& w_{\mu}^{\nu}\left(k_{1}, k_{2}, k_{3}\right)=\frac{\delta_{k_{1} k_{2} k_{3} \mu}^{k_{1} k_{2} k_{3} \nu}}{\Delta\left(k_{1}, k_{2}, k_{3}\right)}=n_{1 \mu} n_{1}^{\nu}=\frac{\varepsilon_{k_{1} k_{2} k_{3} \mu} \varepsilon^{k_{1} k_{2} k_{3} \nu}}{\Delta\left(k_{1}, k_{2}, k_{3}\right)} .
\end{aligned}
$$

In applications to one loop calculations, it is often needed to write the loop momentum $l$ as a linear combination of the van Neerven - Vermaseren basis vectors for a particular graph with the denominator factors $d_{1}, d_{2}, \ldots d_{R}$. The denominators are given by $d_{i}=\left(l+q_{i}\right)^{2}-m_{i}^{2}$ and the inflow momenta read $k_{i}=$ $q_{i}-q_{i-1}$. The decomposition is obtained by contracting the loop momentum with the metric tensor given in Eq. (3.23)

$$
l^{\mu}=\sum_{i=1}^{D_{P}}\left(l \cdot k_{i}\right) v_{i}^{\mu}+\sum_{i=1}^{D_{T}}\left(l \cdot n_{i}\right) n_{i}^{\mu} .
$$


Using the identity

$$
l \cdot k_{i}=\frac{1}{2}\left[d_{i}-d_{i-1}-\left(q_{i}^{2}-m_{i}^{2}\right)+\left(q_{i-1}^{2}-m_{i-1}^{2}\right)\right],
$$

we find

$$
l^{\mu}=V_{R}^{\mu}+\frac{1}{2} \sum_{i=1}^{D_{P}}\left(d_{i}-d_{i-1}\right) v_{i}^{\mu}+\sum_{i=1}^{D_{T}}\left(l \cdot n_{i}\right) n_{i}^{\mu},
$$

where $d_{0}=d_{R}, m_{0}=m_{R}$ and

$$
V_{R}^{\mu}=-\frac{1}{2} \sum_{i=1}^{D_{P}}\left(\left(q_{i}^{2}-m_{i}^{2}\right)-\left(q_{i-1}^{2}-m_{i-1}^{2}\right)\right) v_{i}^{\mu} .
$$

As an illustration of this procedure, we explicitly give the loop-momentum decomposition in two cases. The first example concerns the five-point function in four dimensions, so that $D=4$ and $R=5$. We derive

$$
\begin{aligned}
l^{\mu} & =V_{5}^{\mu}+\frac{1}{2}\left(d_{1}-d_{5}\right) v_{1}^{\mu}+\frac{1}{2}\left(d_{2}-d_{1}\right) v_{2}^{\mu} \\
& +\frac{1}{2}\left(d_{3}-d_{2}\right) v_{3}^{\mu}+\frac{1}{2}\left(d_{4}-d_{3}\right) v_{4}^{\mu}, \\
V_{5}^{\mu} & =-\frac{1}{2}\left(q_{1}^{2}-q_{5}^{2}-m_{1}^{2}+m_{5}^{2}\right) v_{1}^{\mu}-\frac{1}{2}\left(q_{2}^{2}-q_{1}^{2}-m_{2}^{2}+m_{1}^{2}\right) v_{2}^{\mu} \\
& -\frac{1}{2}\left(q_{3}^{2}-q_{2}^{2}-m_{3}^{2}+m_{2}^{2}\right) v_{3}^{\mu}-\frac{1}{2}\left(q_{4}^{2}-q_{3}^{2}-m_{4}^{2}+m_{3}^{2}\right) v_{4}^{\mu} .
\end{aligned}
$$

Similarly, for a three-point function in four dimensions $D=4$ and $R=3$. We obtain

$$
\begin{aligned}
l^{\mu} & =V_{3}^{\mu}+\frac{1}{2}\left(d_{1}-d_{3}\right) v_{1}^{\mu}+\frac{1}{2}\left(d_{2}-d_{1}\right) v_{2}^{\mu}+\left(l \cdot n_{1}\right) n_{1}^{\mu}+\left(l \cdot n_{2}\right) n_{2}^{\mu}, \\
V_{3}^{\mu} & =-\frac{1}{2}\left(q_{1}^{2}-q_{3}^{2}-m_{1}^{2}+m_{3}^{2}\right) v_{1}^{\mu}-\frac{1}{2}\left(q_{2}^{2}-q_{1}^{2}-m_{2}^{2}+m_{1}^{2}\right) v_{2}^{\mu} .
\end{aligned}
$$

We conclude this Section with a few comments. We note that if the number of inflow momenta $R$ exceeds the dimensionality of space-time $D$, the decomposition of the loop momentum into the van Neerven - Vermaseren basis may be used to prove that the $D+m$ point functions $m \geq 1$ can all be written as linear combinations of the $D$-point functions. We will show an example of this in the next Section. We also remark that if we set $q_{0}=0$ we can choose to parametrize the loop momenta using the coordinate system of the $q_{i}$ vectors with its associated dual coordinate basis $v_{i}\left(q_{1}, q_{2}, \ldots, q_{D_{P}}\right)$ as given by Eqs. $(3.18,3.20)$. We note that $\Delta\left(k_{1}, k_{2}, \ldots, k_{D_{P}}\right)=\Delta\left(q_{1}, q_{2}, \ldots, D_{P}\right)$. Similarly the projection operator onto the transverse space remains the same. The identity Eq. (3.27) and all those relations that depend on this identity will be modified accordingly. We conclude that it is possible to change from one basis, to another linearly dependent one at essentially no cost. This fact can be useful in numerical applications. Finally, we emphasize that both versions of the van 
Neerven - Vermaseren basis allow us to include the unitarity constraints without resorting to the spinor-helicity formalism, which is most often used in analytic calculations with massless particles. By avoiding the spinor-helicity formalism, the method can be used in computations with massive internal particles, where the mass can be either real or complex-valued.

\section{Reduction at the integrand level in two dimensions}

Analytic calculations in four dimensions require significant algebraic effort, that often obscures the conceptual aspects of reduction techniques using the van Neerven-Vermaseren basis. The amount of algebra can be kept to a minimum by working in two-dimensional space-time. This section presents a number of two dimensional examples. In what follows we first show that, in two dimensions, the three-point function can be always written as a linear combination of twopoint functions. After that, we express a rank-two, two-point function in terms of scalar two-point functions and tadpoles using unitarity-based ideas. Finally, we discuss a physical example in two-dimensional space-time where the rational part plays an important role.

\subsection{Reduction of a scalar triangle}

We consider a scalar three-point function in two-dimensional space-time,

$$
I_{3}=\int \frac{\mathrm{d}^{2} l}{(2 \pi)^{2}} \mathcal{I}_{3}
$$

and focus specifically on the integrand given by,

$$
\mathcal{I}_{3}=\frac{1}{d_{0} d_{1} d_{2}}
$$

where $l$ is the loop momentum, $d_{i}=\left(l+q_{i}\right)^{2}-m_{i}^{2}, i \in[0,1,2]$ and $q_{0}=0$. To show that, in two dimensions, the three-point function is given by the linear combination of the two-point functions, we use the fact that the loop momentum can be written as a linear combination of the vectors $q_{1,2}$. It is convenient to employ the van Neerven - Vermaseren basis for this purpose. For the case of a three-point function in two dimensions, the dimensionality of space-time and the dimensionality of the physical space coincide. Therefore, we write

$$
l^{\mu}=v_{1}^{\mu}\left(l \cdot q_{1}\right)+v_{2}^{\mu}\left(l \cdot q_{2}\right), \quad v_{1}^{\mu}=\frac{\delta_{q_{1} q_{2}}^{\mu q_{2}}}{\Delta_{2}}, \quad v_{2}^{\mu}=\frac{\delta_{q_{1} q_{2}}^{q_{1} \mu}}{\Delta_{2}},
$$

where, as usual $v_{i} \cdot q_{j}=\delta_{i j}$ and $\Delta_{2}$ is the two-dimensional Gram determinant

$$
\Delta_{2}=q_{1}^{2} q_{2}^{2}-\left(q_{1} \cdot q_{2}\right)^{2} .
$$

We can eliminate the scalar products $l \cdot q_{1,2}$ from Eq. (4.3) using the following equations

$$
l \cdot q_{i}=\frac{1}{2}\left(d_{i}-d_{0}-r_{i}\right), \quad r_{i}=q_{i}^{2}-m_{i}^{2}+m_{0}^{2}, \quad i=1,2 .
$$


To perform this elimination we contract Eq. (4.3) with $l$, use Eq. (4.5) and $l^{2}=d_{0}+m_{0}^{2}$, and write

$$
2\left(d_{0}+m_{0}^{2}\right)=\sum_{i=1}^{2}\left(l \cdot v_{i}\right)\left(d_{i}-d_{0}\right)-\sum_{i=1}^{2}\left(l \cdot v_{i}\right) r_{i} .
$$

We can use the identity

$$
l^{\mu}=g^{\mu \nu} l_{\nu}=\sum_{j=1}^{2} v_{j}^{\mu}\left(q_{j} \cdot l\right),
$$

to rewrite the last term in Eq. (4.6) in the following form

$$
\sum_{i=1}^{2}\left(l \cdot v_{i}\right) r_{i}=\sum_{j=1}^{2}\left(w \cdot v_{j}\right)\left(l \cdot q_{j}\right)=\frac{1}{2} \sum_{j=1}^{2}\left(w \cdot v_{j}\right)\left(d_{j}-d_{0}-r_{j}\right),
$$

where the vector $w^{\mu}$ is defined as

$$
w^{\mu}=\sum_{i=1}^{2} v_{i}^{\mu} r_{i}
$$

We use Eq. (4.8) to simplify the last term in Eq. (4.6), and get

$2 d_{0}+2 m_{0}^{2}=\frac{1}{2}\left(\sum_{i}\left[\left(2 l \cdot v_{i}\right)-\left(w \cdot v_{i}\right)\right] d_{i}-\sum_{i}(2 l-w) \cdot v_{i} d_{0}+\sum_{i} r_{i}\left(w \cdot v_{i}\right)\right)$.

Dividing this equation by $d_{0} d_{1} d_{2}$, using $w^{2}=\sum_{i} r_{i}\left(w \cdot v_{i}\right)$, and collecting similar terms, we obtain the reduction formula for the integrand of the three-point function in two-dimensional space-time

$$
\begin{aligned}
\mathcal{I}_{3}=\frac{1}{\left(4 m_{0}^{2}-w^{2}\right)}\{ & \frac{2\left(l \cdot v_{1}\right)-\left(w \cdot v_{1}\right)}{d_{0} d_{2}}+\frac{2\left(l \cdot v_{2}\right)-\left(w \cdot v_{2}\right)}{d_{0} d_{1}} \\
& \left.-\frac{4+(2 l-w) \cdot\left(v_{1}+v_{2}\right)}{d_{1} d_{2}}\right\} .
\end{aligned}
$$

Later on, we will discuss in detail the parametrization of the integrand due to Ossola, Pittau and Papadopoulos (OPP), but Eq. (4.11) provides a first example of the OPP parametrization applied to the case where the dimensionality of space-time and the dimensionality of the physical space coincide, $R=D=2$. Indeed, the numerator of each of the three terms on the right hand side of Eq. (4.11) is a rank-one tensor of the form $b_{0}+b_{1}(n \cdot l)$. This tensor is special since the loop momentum $l$ appears multiplied by a vector $n$, that is orthogonal to the reference vector in the denominator of each individual term. This is evident for the first two terms on the right-hand side of Eq. (4.11) since $q_{2} \cdot v_{1}=q_{1} \cdot v_{2}=0$, 
but it is also true in the third term. Indeed, shifting the loop momentum in the third term in Eq. (4.11) $l \rightarrow \tilde{l}=l-q_{1}$, we observe that the reference momentum in the denominator becomes $q_{2}-q_{1}$. Since $\left(q_{2}-q_{1}\right) \cdot\left(v_{2}+v_{1}\right)=0$, we have demonstrated our assertion.

To see why the special form of the tensor is important, we compute the three-point function by performing the integral over the loop momentum

$$
I_{3}=\int \frac{\mathrm{d}^{2} l}{(2 \pi)^{2}} \mathcal{I}_{3}
$$

In spite of the fact that the right hand side of Eq. (4.12) contains an integral of a rank-one tensor, cf. Eq. (4.11), the integration is trivial. Indeed, the loop momentum is always contracted with the basis vector of the transverse space and the corresponding angular integrals vanish by symmetry. We obtain ${ }^{4}$

$$
I_{3}=\frac{(-1)}{\left(4 m_{0}^{2}-w^{2}\right)}\left\{\left(w \cdot v_{1}\right) I_{02}+\left(w \cdot v_{2}\right) I_{01}+\left(2-w \cdot\left(v_{1}+v_{2}\right)\right) I_{12}\right\},
$$

where $I_{i j}=\int \frac{\mathrm{d}^{2} l}{(2 \pi)^{2}} \frac{1}{d_{i} d_{j}}$ is a two-point function. Eq. (4.13) completes our proof that in the two-dimensional space-time, the three-point function is given by a linear combination of two-point functions. It is important to realize that this result generalizes. Indeed, any $N$-point function, for $N>D$, where $D$ is the dimensionality of space-time, can be written as a linear combination of the $D$-point functions. Finally, we will see in what follows that the two principal ideas behind the reduction process just outlined - the use of the van Neerven - Vermaseren basis and the reduction by an algebraic integration over the transverse space after establishing the parametric form of the integrand can be easily extended to higher-dimensional spaces and higher-point functions, making it a powerful tool for one-loop computations.

\subsection{Reduction of a rank-two two-point function}

Our next two-dimensional example concerns the reduction of a rank-two twopoint function using van Neerven - Vermaseren basis. Consider an integrand given by

$$
\mathcal{I}\left(k, m_{1}, m_{2}\right)=\frac{(\hat{n} \cdot l)^{2}}{d_{1} d_{2}},
$$

where $d_{1}=l^{2}-m_{1}^{2}, d_{2}=(l+k)^{2}-m_{2}^{2}, \hat{n} \cdot k=0, k^{2} \neq 0$ and $\hat{n}^{2}=1$. Because of the projection onto $\hat{n}$, the momentum $l$ in the numerator in Eq. (4.14) lies in the transverse space. We want to express this integral in terms of scalar integrals.

Note that in contrast to the three-point function considered in the preceding Section, the rank-two two-point function in two dimensions has an ultraviolet

\footnotetext{
${ }^{4}$ It is convenient to shift of the loop momentum $l \rightarrow l-q_{1}$ in the last term of Eq. (4.11), so that the remaining vector integral vanishes by symmetry.
} 
divergence. We regularize this divergence by continuing the loop momentum to $D=2-2 \epsilon$ dimensions and begin by constructing the van Neerven - Vermaseren basis. As the basis vector of the physical space, we take

$$
n^{\mu}=\frac{k^{\mu}}{\sqrt{k^{2}}}, \quad n^{2}=1
$$

We choose $\hat{n}$ to be the basis vector of the transverse space, which is allowed since $n$ and $\hat{n}$ are orthogonal, $n \cdot \hat{n}=0$. As the consequence of the completeness relation, the two vectors satisfy

$$
n^{\mu} n^{\nu}+\hat{n}^{\mu} \hat{n}^{\nu}=g_{(2)}^{\mu \nu},
$$

where $g_{(2)}^{\mu \nu}$ is the two-dimensional metric tensor. Contracting this equation with the loop momentum, we obtain

$$
(\hat{n} \cdot l)^{2}=l_{(2)}^{2}-(n \cdot l)^{2}=l_{(2)}^{2}-\frac{(l \cdot k)^{2}}{k^{2}} .
$$

Because $l$ is a $d$-dimensional vector, we can decompose it as

$$
l^{\mu}=(l \cdot n) n^{\mu}+(l \cdot \hat{n}) \hat{n}^{\mu}+n_{\epsilon}^{\mu}\left(l \cdot n_{\epsilon}\right),
$$

where $n_{\epsilon}$ is the unit vector that parametrizes the $(D-2)$-dimensional vector space. It follows that the square of the $d$-dimensional loop momentum can be written as

$$
l^{2}=l_{(2)}^{2}+\left(n_{\epsilon} \cdot l\right)^{2}=l_{(2)}^{2}+\mu^{2},
$$

where $\mu^{2}=\left(n_{\epsilon} \cdot l\right)^{2}$ is introduced. To proceed further, we express various scalar products through inverse Feynman propagators $d_{1,2}$

$$
l_{(2)}^{2}=d_{1}+m_{1}^{2}-\mu^{2}, \quad 2 l \cdot k=d_{2}-d_{1}-r_{1}^{2},
$$

and use Eqs. $(4.17,4.19)$ to obtain

$$
\frac{(\hat{n} \cdot l)^{2}}{d_{1} d_{2}}=-\frac{\left(\lambda^{2}+\mu^{2}\right)}{d_{1} d_{2}}+\frac{1}{4 k^{2}}\left[\frac{r_{1}^{2}-2 l \cdot k}{d_{1}}+\frac{r_{2}^{2}+2 l \cdot k+2 k^{2}}{d_{2}}\right] .
$$

In Eqs. (4.20,4.21), we use the following short-hand notations

$$
\begin{aligned}
& r_{1}^{2}=k^{2}+m_{1}^{2}-m_{2}^{2}, \quad r_{2}^{2}=k^{2}+m_{2}^{2}-m_{1}^{2}, \\
& \lambda^{2}=\frac{k^{4}-2 k^{2}\left(m_{1}^{2}+m_{2}^{2}\right)+\left(m_{1}^{2}-m_{2}^{2}\right)^{2}}{4 k^{2}} .
\end{aligned}
$$

Even if we did not know the result displayed in Eq. (4.21), we could still argue on general grounds that the integrand can be written as

$$
\begin{aligned}
\frac{(\hat{n} \cdot l)^{2}}{d_{1} d_{2}}= & \frac{b_{0}+b_{1}(\hat{n} \cdot l)+b_{2}\left(n_{\epsilon} \cdot l\right)^{2}}{d_{1} d_{2}}+\frac{a_{1,0}+a_{1,1}(n \cdot l)+a_{1,2}(\hat{n} \cdot l)}{d_{1}} \\
& +\frac{a_{2,0}+a_{2,1}(n \cdot l)+a_{2,2}(\hat{n} \cdot l)}{d_{2}} .
\end{aligned}
$$


We will explain in Section 5 where this parametrization comes from. Here, we compare terms in Eq. (4.21) and Eq. (4.23) and obtain

$$
\begin{aligned}
& b_{0}=-\lambda^{2}, \quad b_{1}=0, \quad b_{2}=-1, \\
& a_{1,0}=\frac{r_{1}^{2}}{4 k^{2}}, \quad a_{1,1}=-\frac{1}{2 \sqrt{k^{2}}}, \quad a_{1,2}=0, \\
& a_{2,0}=\frac{r_{2}^{2}}{4 k^{2}}+\frac{1}{2}, \quad a_{2,1}=\frac{1}{2 \sqrt{k^{2}}}, \quad a_{2,2}=0 .
\end{aligned}
$$

It is instructive to rederive Eq. (4.24) using an alternative procedure. This procedure is important because it generalizes to four-dimensions, without modification, and because it shows how the reduction techniques are connected to unitarity. We begin by multiplying both sides of Eq. (4.23) by $d_{1}, d_{2}$ and obtain

$$
\begin{aligned}
(\hat{n} \cdot l)^{2} & =\left[b_{0}+b_{1}(\hat{n} \cdot l)+b_{2}\left(n_{\epsilon} \cdot l\right)^{2}\right]+\left[a_{1,0}+a_{1,1}(n \cdot l)+a_{1,2}(\hat{n} \cdot l)\right] d_{2} \\
& +\left[a_{2,0}+a_{2,1}(n \cdot l)+a_{2,2}(\hat{n} \cdot l)\right] d_{1} .
\end{aligned}
$$

We would like to use Eq. (4.25) to find all the $b$ - and $a$-coefficients. Since there are nine unknowns, we can evaluate Eq. (4.25) for nine different values of the loop momentum $l$, invert the nine-by-nine matrix and find the coefficients. While this procedure does, indeed, provide a solution to the problem, it requires inverting a large matrix and is therefore impractical. A better algorithm exploits the fact that, under special choices of the loop momentum $l$ in Eq. (4.25), the matrix to invert becomes block-diagonal.

To see how this works, we first describe a procedure to compute the $b$ coefficients only. To project the right hand side of Eq. (4.25) onto $b$-coefficients, we choose the loop momentum $l$ to satisfy $d_{1}(l)=d_{2}(l)=0$. For the moment, consider the loop momentum $l$ that satisfies those constraints and, simultaneously, has zero projection on the $d$-dimensional space, $n_{\epsilon} \cdot l=0$. We find that there are just two loop momenta $l$ that satisfy those constraints; they can be written as

$$
l_{c}^{ \pm}=\alpha_{c} n \pm i \beta_{c} \hat{n}
$$

where

$$
\alpha_{c}=-\frac{r_{1}^{2}}{2 \sqrt{k^{2}}}, \quad \beta_{c}=\lambda .
$$

The parameters $r_{1}$ and $\lambda$ are shown in Eq. (4.22). We substitute these two solutions into Eq. (4.25) and obtain two equations for the coefficients $b_{0,1}$

$$
b_{0}+b_{1} \hat{n} \cdot l_{c}^{+}=-\lambda^{2}, \quad b_{0}+b_{1} \hat{n} \cdot l_{c}^{-}=-\lambda^{2} .
$$

It follows that $b_{0}=-\lambda^{2}$ and $b_{1}=0$, in agreement with Eq. (4.24).

To find $b_{2}$ we proceed along similar lines but we require that the scalar product $l \cdot n_{\epsilon}$ does not vanish. Since the conditions $d_{1}=0, d_{2}=0$ are equivalent to $2 l \cdot k+r_{1}^{2}=0, l^{2}=m_{1}^{2}$, the loop momentum that satisfies those constraints is the same as in Eq. (4.26), up to a change $\hat{n} \rightarrow n_{\epsilon}$,

$$
l^{ \pm}=\alpha_{c} n \pm i \beta_{c} n_{\epsilon} .
$$


Substituting $l^{ \pm}$into Eq. (4.25) and using $b_{0}=-\lambda^{2}, b_{1}=0$, we obtain

$$
0=\left(1+b_{2}\right) \lambda^{2}
$$

which implies that $b_{2}=-1$, in agreement with Eq. (4.24).

The next step is to identify the coefficients of the tadpoles in Eq. (4.25). We will focus on a set $a_{1,0}, a_{1,1}, a_{1,2}$. We can project Eq. (4.25) on these coefficients by choosing the loop momentum for which $d_{1}$ vanishes but $d_{2}$ is different from zero. Note that no $l \cdot n_{\epsilon}$ terms are needed to find the $a$-coefficients. As a consequence, we can work with a two-dimensional loop momentum

$$
l_{1}=\gamma_{1} n+\gamma_{2} \hat{n} \text {. }
$$

The equation $d_{1}\left(l_{1}\right)=0$ implies $\gamma_{1}^{2}+\gamma_{2}^{2}=m_{1}^{2}$, so that $\gamma_{1}, \gamma_{2}$ lie on a circle of a radius $m_{1}$. Substituting $l_{1}$ into Eq. (4.25), we find

$$
\gamma_{2}^{2}=-\lambda^{2}+\left(2 \sqrt{k^{2}} \gamma_{1}+r_{1}^{2}\right)\left(a_{1,0}+a_{1,1} \gamma_{1}+a_{1,2} \gamma_{2}\right)
$$

To solve Eq. (4.32), we choose $\gamma_{1}=0, \gamma_{2}= \pm m_{1}$ and obtain two equations

$$
a_{1,0} \pm a_{1,2} m_{1}=\frac{m_{1}^{2}+\lambda^{2}}{r_{1}^{2}}=\frac{r_{1}^{2}}{4 k^{2}}
$$

Hence, it follows that $a_{1,0}=r_{1}^{2} /\left(4 k^{2}\right)$ and $a_{1,2}=0$, in agreement with Eq. (4.24). To find $a_{1,1}$, we choose $\gamma_{2}=0, \gamma_{1}=m_{1}$, solve Eq. (4.32) and obtain $a_{1,1}=$ $-\left(4 k^{2}\right)^{-1 / 2}$.

We can determine coefficients $a_{2,0}, a_{2,1}, a_{2,2}$ in the same manner, by choosing the loop momentum that satisfies $d_{2}(l)=0$. The calculation is similar to the one performed above and for this reason we do not present it here. We emphasize that the procedure that we just explained implies that, for the reduction of oneloop integrals to a set of scalar integrals, we need to know integrands at special values of the loop momenta, for which at least one of the inverse Feynman propagators that contributes to a particular diagram, vanishes. Since zeros of Feynman denominators correspond to situations when virtual particles go on their mass shells, the connection between the reduction procedure and the ideas of unitarity begins to emerge.

\subsection{The photon mass in the Schwinger model}

We will conclude our discussion of the two-dimensional physics by emphasizing the role that remnants of ultraviolet regularization play in one-loop calculations. Such terms are known as the rational part; an example is the $\left(l \cdot n_{\epsilon}\right)^{2} /\left(d_{1} d_{2}\right)$ term in Eq. (4.23). Note that those terms cannot be found by studying the integrand as a function of the four-dimensional loop momentum; the analytic continuation of the loop momentum to $d$-dimensions is crucial for the identification of those terms.

We will discuss the rational part extensively in the following sections. However, the importance of those terms can be illustrated by considering twodimensional QED - the Schwinger model [27]. The Schwinger model is exactly 
solvable; it is often used to illustrate a variety of interesting phenomena in quantum field theory. Among these phenomena is the well-known result that the photon acquires a dynamical mass. As we now show, this mass is generated by the rational part of the photon vacuum polarization at one loop. To this end, consider the photon vacuum polarization function due to a loop of massless fermions,

$$
\Pi_{12}=-e^{2} \int \frac{\mathrm{d}^{D} l}{(2 \pi)^{D}} \frac{\operatorname{Tr}\left(\hat{\epsilon}_{1} \hat{l}_{\hat{\epsilon}}(\hat{l}+\hat{k})\right)}{l^{2}(l+k)^{2}},
$$

where $\epsilon_{1}$ and $\epsilon_{2}$ are the two-dimensional polarization vectors of the virtual photon, $D=2-2 \epsilon$ and $\hat{p} \equiv \gamma_{\mu} p^{\mu}$. The reason we introduce the polarization vectors in the above equation is to simplify the bookkeeping of the two- and $d$-dimensional Lorentz indices in what follows.

It is clear from the discussion in the previous Section, that $\Pi_{12}$ can be written as a linear combination of the scalar one- and two-point functions. The one-point functions with massless fermion propagators vanish in dimensional regularization. Therefore, the non-vanishing contribution to $\Pi_{12}$ can only come from the scalar two-point function. According to the previous Section, a reduction coefficient in this case can be obtained by studying the integrand in Eq. (4.34) for values of the loop momentum $l$ such that the two inverse Feynman propagators vanish

$$
l^{2}=0, \quad(l+k)^{2}=0 .
$$

Since such contribution corresponds to both intermediate particles in the loop being on the mass shell, we refer to such a contribution as the "double cut". We can parametrize the loop-momentum using the van Neerven - Vermaseren basis introduced in Sect.3

$$
l^{\mu}=(l \cdot k) v_{1}^{\mu}+l_{\perp}^{\mu}+\left(l \cdot n_{\epsilon}\right) n_{\epsilon}^{\mu},
$$

where $v_{1}^{\mu}=k^{\mu} / k^{2}, l_{\perp}^{\mu}=(l \cdot n) n^{\mu}$ is a two-dimensional vector orthogonal to $k$ and $n_{\epsilon}^{\mu}$ denotes a unit vector that parametrizes the $(D-2)$ dimensions. Solving the two constraints, we find

$$
l^{\mu}=-\frac{1}{2} k^{\mu}+l_{\perp}^{\mu}+\left(l \cdot n_{\epsilon}\right) n_{\epsilon}^{\mu}, \quad l_{\perp}^{2}+\left(l \cdot n_{\epsilon}\right)^{2}=-\frac{k^{2}}{4},
$$

with $k \cdot l_{\perp}=n_{\epsilon} \cdot k=n_{\epsilon} \cdot l_{\perp}=0$. Calculating the trace in Eq. (4.34), substituting solutions from Eq. (4.37) and disregarding terms that are linear in $l_{\perp}$ or $n_{\epsilon}$, we obtain

$$
\begin{gathered}
\Pi_{12}=-2 e^{2} \int \frac{\mathrm{d}^{D} l}{(2 \pi)^{D}} \frac{1}{l^{2}(l+k)^{2}}\left[2\left(l_{\perp} \cdot \epsilon_{1}\right)\left(l_{\perp} \cdot \epsilon_{2}\right)\right. \\
\left.+\frac{k^{2}}{2}\left(\epsilon_{1} \cdot \epsilon_{2}-\frac{\left(\epsilon_{1} \cdot k\right)\left(\epsilon_{2} \cdot k\right)}{k^{2}}\right)\right] .
\end{gathered}
$$


Because $l^{2}=l_{\perp}^{2}+\ldots$ and $(l+k)^{2}=l_{\perp}^{2}+\ldots$, the angular integration over the direction of $l_{\perp}$ is trivial and we can write

$$
\begin{aligned}
\left(l_{\perp} \cdot \epsilon_{1}\right)\left(l_{\perp} \cdot \epsilon_{2}\right) & \rightarrow l_{\perp}^{2}\left(\epsilon_{1} \cdot \epsilon_{2}-\frac{\left(\epsilon_{1} \cdot k\right)\left(\epsilon_{2} \cdot k\right)}{k^{2}}\right) \\
& =\left(-\frac{k^{2}}{4}-\left(l \cdot n_{\epsilon}\right)^{2}\right)\left(\epsilon_{1} \cdot \epsilon_{2}-\frac{\left(\epsilon_{1} \cdot k\right)\left(\epsilon_{2} \cdot k\right)}{k^{2}}\right)
\end{aligned}
$$

Using this result in Eq. (4.38), we derive a simple formula for the polarization operator

$$
\Pi_{12}=4 e^{2}\left(\epsilon_{1} \cdot \epsilon_{2}-\frac{\left(\epsilon_{1} \cdot k\right)\left(\epsilon_{2} \cdot k\right)}{k^{2}}\right) \int \frac{\mathrm{d}^{D} l}{(2 \pi)^{D}} \frac{\left(l \cdot n_{\epsilon}\right)^{2}}{l^{2}(l+k)^{2}} .
$$

Eq. (4.40) shows that the photon vacuum polarization in two-dimensional QED is non-vanishing only because of a remnant of the ultraviolet regularization. Using modern jargon, we say that it is purely rational. We compute the integral in the limit $D \rightarrow 2$,

$$
\int \frac{\mathrm{d}^{D} l}{(2 \pi)^{D}} \frac{\left(l \cdot n_{\epsilon}\right)^{2}}{l^{2}(l+k)^{2}}=\frac{i}{4 \pi},
$$

and obtain

$$
\Pi_{12}=i \frac{e^{2}}{\pi}\left(\epsilon_{1} \cdot \epsilon_{2}-\frac{\left(\epsilon_{1} \cdot k\right)\left(\epsilon_{2} \cdot k\right)}{k^{2}}\right) .
$$

A resummation of the vacuum polarization contributions shown in Eq. (4.42) gives the well-known massive photon propagator in the Schwinger model [27]

$$
\Pi_{\mu \nu}=\frac{-i}{k^{2}-m_{\gamma}^{2}}\left(g_{\mu \nu}-\frac{k_{\mu} k_{\nu}}{k^{2}}\right), \quad m_{\gamma}^{2}=\frac{e^{2}}{\pi} .
$$

Hence, the photon mass $m_{\gamma}^{2}=e^{2} / \pi$ comes entirely from the rational part of the photon vacuum polarization diagram in two dimensions.

\section{Reduction at the integrand level in $D$-dimensions}

In this Section, we generalize the two-dimensional reduction procedure described in the previous Section to $D$-dimensional space-time. We are ultimately interested in the limit $D \rightarrow 4$.

\subsection{Parametrization of the integrand}

We begin with the observation that, in any renormalizable quantum field theory, the rank of the one-loop tensor integrals that appear does not exceed the number of external lines. Therefore, we will only be concerned with the reduction of one-loop integrals of restricted rank, e.g. the rank-five or less for five-point functions, rank-four or less for four-point functions and so on. 
We would like to establish a simple parametrization of one-loop integrands, following a seminal suggestion by Ossola, Papadopoulos and Pittau [21]. It reads

$$
\begin{aligned}
I_{N} & =\int \frac{\mathrm{d}^{D} l}{(2 \pi)^{D}} \frac{\mathrm{Num}(l)}{\prod_{i} d_{i}(l)}=\int \frac{\mathrm{d}^{D} l}{(2 \pi)^{D}} \frac{1}{\prod_{i} d_{i}(l)} \times\{ \\
& +\sum_{i_{1}, i_{2}, i_{3}, i_{4}, i_{5}} \tilde{e}_{i_{1}, i_{2}, i_{3}, i_{4}, i_{5}}(l) \prod_{j \neq\left[i_{1}, i_{2}, i_{3}, i_{4}, i_{5}\right]} d_{j}(l) \\
& +\sum_{i_{1}, i_{2}, i_{3}, i_{4}} \tilde{d}_{i_{1}, i_{2}, i_{3}, i_{4}}(l) \prod_{j \neq\left[i_{1}, i_{2}, i_{3}, i_{4}\right]} d_{j}(l) \\
& +\sum_{i_{1}, i_{2}, i_{3}} \tilde{c}_{i_{1}, i_{2}, i_{3}}(l) \prod_{j \neq\left[i_{1}, i_{2}, i_{3}\right]} d_{j}(l) \\
& \left.+\sum_{i_{1}, i_{2}} \tilde{b}_{i_{1}, i_{2}}(l) \prod_{j \neq\left[i_{1}, i_{2}\right]} d_{j}(l)+\sum_{i_{1}} \tilde{a}_{i_{1}}(l) \prod_{j \neq i_{1}} d_{j}(l)\right\} .
\end{aligned}
$$

The index $i$ runs over all possible inverse Feynman propagators $d_{i}$. Similarly, the index $j$ runs over all inverse Feynman propagators, except those explicitly excluded. The important feature of this parametrization is that all inverse propagators $d_{i}(l)$ on the right hand side appear in the first power, i.e. there are no terms of the form $d_{i}^{2}(l)$ for any $i$. In the spirit of the previous section, this allows us to project on different $\tilde{e}, \tilde{d}, \tilde{c}, \tilde{b}$ and $\tilde{a}$-functions, by considering loop momenta that nullify different sets of inverse propagators.

We will discuss first the reduction of a rank-five five-point function; the general case then easily follows. To this end, we consider $d_{i}(l)=\left(l+q_{i}\right)^{2}-m_{i}^{2}$, $i=0, \ldots, 4, q_{0}=0$ and assume that the numerator function reads

$$
\operatorname{Num}(l)=N_{5}(l)=\prod_{i=1}^{5} u_{i} \cdot l,
$$

where $u_{i}$ are some external four-dimensional vectors.

As the first step in the reduction procedure, we find the reduction coefficients of the five-point function, $\tilde{e}_{01234}$. To accomplish this, we construct the van Neerven - Vermaseren basis out of four vectors $q_{i}$ and decompose the loop momentum

$$
l^{\mu}=\sum_{i=1}^{4}\left(l \cdot q_{i}\right) v_{i}^{\mu}+\left(l \cdot n_{\epsilon}\right) n_{\epsilon}^{\mu} .
$$

The scalar products $l \cdot q_{i}$ are expressed in terms of inverse Feynman propagators

$$
l \cdot q_{i}=\frac{1}{2}\left(d_{i}-d_{0}-\left(q_{i}^{2}-m_{i}^{2}+m_{0}^{2}\right)\right) .
$$


Since $u_{5} \cdot n_{\epsilon}=0$, we can rewrite Eq. (5.2) as

$$
\begin{aligned}
& N_{5}(l)=\left(\prod_{i}^{4} u_{i} \cdot l\right)\left(u_{5} \cdot l\right)=\frac{1}{2} \sum_{j=1}^{4}\left(u_{5} \cdot v_{j}\right)\left(\prod_{i}^{4} u_{i} \cdot l\right)\left(d_{j}-d_{0}\right) \\
& -\frac{1}{2} \sum_{j=1}^{4}\left(u_{5} \cdot v_{j}\right)\left(\prod_{i}^{4} u_{i} \cdot l\right)\left(q_{j}^{2}-m_{j}^{2}+m_{0}^{2}\right) .
\end{aligned}
$$

Upon dividing the numerator function by the product of inverse Feynman propagators $d_{0} d_{1} d_{2} d_{3} d_{4}$, we find that the first term on the right-hand-side of Eq. (5.5), produces a collection of rank-four four-point functions and the second term a rank-four five-point function. We now repeat the same procedure with the rank-four five-point function and conclude that it can be expressed through a combination of rank-three four-point functions and the rank-three five point function. Whenever, as a result of these manipulations, the propagator $d_{0}$ cancels, it is possible to shift the loop-momentum to bring the integrand to the standard form. We can clearly continue this procedure until we are left with a scalar five-point function and a collection of four-point functions of the ranks from zero (scalar) to four (maximal). Hence, we have established that the function $\tilde{e}_{01234}(l)$ in Eq. (5.1) is $l$-independent

$$
\tilde{e}_{01234}(l)=\tilde{e}_{01234}^{(0)} .
$$

In the course of the procedure described above, the highest rank integral left unreduced is the rank-four four-point function. We now discuss how it can be reduced. For definiteness, we consider the four-point function with four propagators $d_{0}, d_{1}, d_{2}, d_{3}$, but our discussion can be applied to any other fourpoint function, by the appropriate re-definition of the propagator momenta and masses. We construct van Neerven - Vermaseren basis vectors out of the three momenta $q_{1}, q_{2}, q_{3}$. The physical space in this case is three-dimensional and the transverse space is one-dimensional. We parametrize the transverse space by the unit vector $n_{4}$.

The decomposition in terms of van Neerven - Vermaseren basis then reads

$$
l^{\mu}=\sum_{i=1}^{3} v_{i}^{\mu}\left(l \cdot q_{i}\right)+\left(l \cdot n_{4}\right) n_{4}^{\mu}+\left(l \cdot n_{\epsilon}\right) n_{\epsilon}^{\mu} .
$$

Using this parametrization we can write the numerator of the four-point function as,

$$
\begin{aligned}
& N_{4}(l)=\left(\prod_{i}^{3} u_{i} \cdot l\right)\left(u_{4} \cdot l\right)=\frac{1}{2} \sum_{j=1}^{3} u_{4} \cdot v_{j}\left(\prod_{i}^{3} u_{i} \cdot l\right)\left(d_{j}-d_{0}\right) \\
& -\frac{1}{2} \sum_{j=1}^{3} u_{4} \cdot v_{j}\left(q_{j}^{2}-m_{j}^{2}+m_{0}^{2}\right) \prod_{i}^{3} u_{i} \cdot l+\left(l \cdot n_{4}\right)\left(u_{4} \cdot n_{4}\right) \prod_{i}^{3} u_{i} \cdot l .
\end{aligned}
$$


The first two terms on the right-hand side are considered "reduced", since they are rank-three three-point and four-point functions. The last term, however, is a rank-four four-point function, and so it does not appear that we gained anything. To demonstrate that we, actually, did gain something, we take the last term in Eq. (5.8) and repeat the reduction procedure described above. It is clear that a variety of terms will be produced, most of lower-point or lower-rank type, and the only term that we should consider as "not-reduced" reads

$$
\left(\prod_{i}^{3} u_{i} \cdot l\right)\left(l \cdot n_{4}\right) \rightarrow\left(\prod_{i}^{2} u_{i} \cdot l\right)\left(l \cdot n_{4}\right)^{2} .
$$

We simplify it by examining the square of the loop momentum $l$. Using the decomposition in terms of van Neerven - Vermaseren basis, Eq. (5.7) and the relations $2 l \cdot q_{i}=\left(d_{i}-d_{0}-q_{i}^{2}+m_{i}^{2}-m_{0}^{2}\right)$ and $l^{2}=d_{0}+m_{0}^{2}$, we find

$$
\left(l \cdot n_{4}\right)^{2}=-\left(l \cdot n_{\epsilon}\right)^{2}+\text { constant terms }+\mathcal{O}\left(d_{0}, d_{1}, d_{2}, d_{3}\right) .
$$

Terms dubbed "constant" in the above formula contribute (after multiplication by $\left(u_{1} \cdot l\right)\left(u_{2} \cdot l\right)$ to rank-two four-point functions while terms that contain at least one inverse Feynman propagator, contribute to three-point functions. The "not-reduced" part of the rank-four four-point function therefore reads

$$
\prod_{i}^{4} u_{i} \cdot l \rightarrow\left(\prod_{i}^{2} u_{i} \cdot l\right)\left(l \cdot n_{4}\right)^{2} \rightarrow\left(\prod_{i}^{2} u_{i} \cdot l\right)\left(l \cdot n_{\epsilon}\right)^{2} .
$$

It is clear that if we repeat the reduction process, we express any tensor fourpoint function integral (of rank not higher than four), through the following numerator function

$$
\tilde{d}_{0123}(l)=\tilde{d}_{0}+\tilde{d}_{1}\left(l \cdot n_{4}\right)+\tilde{d}_{2}\left(l \cdot n_{\epsilon}\right)^{2}+\tilde{d}_{3}\left(l \cdot n_{\epsilon}\right)^{2}\left(l \cdot n_{4}\right)+\tilde{d}_{4}\left(l \cdot n_{\epsilon}\right)^{4},
$$

where the $l$-dependence is shown explicitly. We note that the degree of the polynomial on the right hand side of Eq. (5.12) is the direct consequence of the fact that the highest rank tensor four-point functions that we consider is four. This restriction works well if we deal with renormalizable quantum field theories but it might not be general enough if one-loop calculations with effective field theories are attempted. The extension of the algorithm to those cases is straightforward since the required parametrization of a numerator function of, say, a four-point function will be an extension of Eq. (5.12) to higher rank tensors. It is straightforward to figure out the required extension, following the line of reasoning explained above. Interestingly, such extensions are very economical; for example, we mention that to achieve reduction of a rank-five four-point function, we need only include one additional term $\tilde{d}_{5}\left(l \cdot n_{\epsilon}\right)^{4}\left(l \cdot n_{4}\right)$ in the parametrization of $\tilde{d}_{0123}$ in Eq. (5.12).

We now turn our attention to the three-point functions that are obtained in the course of the reduction of the four-point functions. The highest tensor rank 
we have to care about is three. The physical space is two-dimensional and the transverse space is two-dimensional as well. The loop momentum reads

$$
l^{\mu}=\sum_{i=1}^{2} v_{i}^{\mu}\left(l \cdot q_{i}\right)+\left(l \cdot n_{3}\right) n_{3}^{\mu}+\left(l \cdot n_{4}\right) n_{4}^{\mu}+\left(l \cdot n_{\epsilon}\right) n_{\epsilon}^{\mu} .
$$

We follow the same procedure as already described in the context of five- and four-point functions. The reduced terms will be at most rank-two two-point functions. The irreducible structures read

$$
\begin{aligned}
& \prod_{i=3}^{4}\left(l \cdot u_{i}\right) \rightarrow \sum_{i=3}^{4} c_{1 i}\left(l \cdot n_{i}\right)+\sum_{i=3}^{4} c_{2 i}\left(l \cdot n_{i}\right)^{2}+\sum_{i=3}^{4} c_{3 i}\left(l \cdot n_{i}\right)^{3} \\
& +c_{4}\left(l \cdot n_{4}\right)\left(l \cdot n_{3}\right)+c_{5}\left(l \cdot n_{3}\right)^{2}\left(l \cdot n_{4}\right)+c_{6}\left(l \cdot n_{3}\right)\left(l \cdot n_{4}\right)^{2} .
\end{aligned}
$$

Similar to the case of the four-point function, not all the terms in Eq. (5.14) are independent in the four-dimensional case. To make this dependence explicit, we square $l^{\mu}$ in Eq. (5.13), use $l^{2}=d_{0}+m_{0}^{2}$ and find

$$
\left(l \cdot n_{3}\right)^{2}+\left(l \cdot n_{4}\right)^{2}+\left(l \cdot n_{\epsilon}\right)^{2}=\text { constant terms }+\mathcal{O}\left(d_{0}, d_{1}, d_{2}\right) .
$$

We use this constraint in Eq. (5.14), to trade $\left(l \cdot n_{3}\right)^{2}\left(l \cdot n_{4}\right), \quad\left(l \cdot n_{4}\right)^{2}\left(l \cdot n_{3}\right)$ for $\left(l \cdot n_{\epsilon}\right)^{2}\left(l \cdot n_{4}\right)$ and $\left(l \cdot n_{\epsilon}\right)^{2}\left(l \cdot n_{3}\right)$. Also, given Eq. (5.15), we can use $\left(l \cdot n_{\epsilon}\right)^{2}$ and $\left(l \cdot n_{3}\right)^{2}-\left(l \cdot n_{4}\right)^{2}$ as two independent structures, instead of $\left(l \cdot n_{3}\right)^{2}$ and $\left(l \cdot n_{4}\right)^{2}$. Hence, the parametrization of the function $\tilde{c}_{012}$ becomes

$$
\begin{aligned}
& \tilde{c}_{012}(l)=\tilde{c}_{0}+\tilde{c}_{1}\left(l \cdot n_{3}\right)+\tilde{c}_{2}\left(l \cdot n_{4}\right)+\tilde{c}_{3}\left(\left(l \cdot n_{3}\right)^{2}-\left(l \cdot n_{4}\right)^{2}\right) \\
& +\tilde{c}_{4}\left(l \cdot n_{3}\right)\left(l \cdot n_{4}\right)+\tilde{c}_{5}\left(l \cdot n_{3}\right)^{3}+\tilde{c}_{6}\left(l \cdot n_{4}\right)^{3} \\
& +\tilde{c}_{7}\left(l \cdot n_{\epsilon}\right)^{2}+\tilde{c}_{8}\left(l \cdot n_{\epsilon}\right)^{2}\left(l \cdot n_{3}\right)+\tilde{c}_{9}\left(l \cdot n_{\epsilon}\right)^{2}\left(l \cdot n_{4}\right) .
\end{aligned}
$$

The advantage of this parametrization, compared to Eq. (5.14), is that in four dimensions only $\tilde{c}_{0}$ gives a non-vanishing contribution after integration.

Similar considerations can be used to derive the general parametrization of the two-point and one-point functions. Recall that the highest tensor rank of the two-point function that we consider is two; the highest tensor rank of the one-point function is one. We will not discuss the derivation and just give the results for the numerator functions. The numerator of the two-point function can be written as

$$
\begin{aligned}
\tilde{b}_{01}(l) & =\tilde{b}_{0}+\tilde{b}_{1}\left(l \cdot n_{2}\right)+\tilde{b}_{2}\left(l \cdot n_{3}\right)+\tilde{b}_{3}\left(l \cdot n_{4}\right) \\
& +\tilde{b}_{4}\left(\left(l \cdot n_{2}\right)^{2}-\left(l \cdot n_{4}\right)^{2}\right)+\tilde{b}_{5}\left(\left(l \cdot n_{3}\right)^{2}-\left(l \cdot n_{4}\right)^{2}\right)+\tilde{b}_{6}\left(l \cdot n_{2}\right)\left(l \cdot n_{3}\right) \\
& +\tilde{b}_{7}\left(l \cdot n_{3}\right)\left(l \cdot n_{4}\right)+\tilde{b}_{8}\left(l \cdot n_{2}\right)\left(l \cdot n_{4}\right)+\tilde{b}_{9}\left(l \cdot n_{\epsilon}\right)^{2},
\end{aligned}
$$

while the general parametrization of the numerator of the one-point function for propagator $d_{i}$ is

$$
\tilde{a}_{i}(l)=\tilde{a}_{0}+\tilde{a}_{1}\left(l \cdot n_{1}\right)+\tilde{a}_{2}\left(l \cdot n_{2}\right)+\tilde{a}_{3}\left(l \cdot n_{3}\right)+\tilde{a}_{4}\left(l \cdot n_{4}\right) .
$$

In Eq. (5.18) $\tilde{a}_{0}$ is the relevant reduction coefficient since all other terms integrate to zero. 


\subsection{Computation of the reduction coefficients}

In the previous Section we showed how an integrand of a general one-loop integral in a renormalizable quantum field theory can be parametrized. An important feature of this parametrization is that all $l$-dependent four-dimensional tensors that are present in the coefficients $\tilde{d}_{i_{1} . . i_{4}}, \ldots, \tilde{a}_{i_{1}}$ vanish if angular integration in the transverse space of the respective reduced integral is performed. We will refer to such tensors as "traceless". This feature is very important since it allows us to rewrite Eq. (5.1) in a simplified, fully reduced form

$$
\begin{aligned}
I_{N} & =\int \frac{\mathrm{d}^{D} l}{(2 \pi)^{D}} \frac{\operatorname{Num}(l)}{\prod_{i} d_{i}(l)}=\sum_{i_{1}, i_{2}, i_{3}, i_{4}, i_{5}} \tilde{e}_{i_{1}, i_{2}, i_{3}, i_{4}, i_{5}}^{(0)} I_{i_{1} i_{2} i_{3} i_{4} i_{5}} \\
& +\sum_{i_{1}, i_{2}, i_{3}, i_{4}} \tilde{d}_{i_{1}, i_{2}, i_{3}, i_{4}}^{(0)} I_{i_{1} i_{2} i_{3} i_{4}}+\sum_{i_{1}, i_{2}, i_{3}} \tilde{c}_{i_{1}, i_{2}, i_{3}}^{(0)} I_{i_{1} i_{2} i_{3}} \\
& +\sum_{i_{1}, i_{2}} \tilde{b}_{i_{1}, i_{2}}^{(0)} I_{i_{1} i_{2}}+\sum_{i_{1}} \tilde{a}_{i_{1}}^{(0)} I_{i_{1}}+\mathcal{R} .
\end{aligned}
$$

Eq. (5.19) proves the reduction formula stated in Eq. (2.5). The right hand side of Eq. (5.19) contains scalar integrals multiplied by $l$-independent contributions of the reduction coefficients $\tilde{e}^{(0)}, \tilde{d}^{(0)}, \tilde{b}^{(0)}$, etc. and the "rational" term $\mathcal{R}$ which originates from the integration over the loop momentum of tensorial structures involving $\left(l \cdot n_{\epsilon}\right)$. The rational part $\mathcal{R}$ is given explicitly by the following equation

$$
\mathcal{R}=-\sum_{i_{1}, i_{2}, i_{3}, i_{4}} \frac{\tilde{d}_{i_{1} i_{2} i_{3} i_{4}}^{(4)}}{6}+\sum_{i_{1}, i_{2}, i_{3}} \frac{\tilde{c}_{i_{1} i_{2} i_{3}}^{(7)}}{2}+\sum_{i_{1}, i_{2}}\left[\frac{m_{i_{1}}^{2}+m_{i_{2}}^{2}}{2}-\frac{\left(q_{i_{1}}-q_{i_{2}}\right)^{2}}{6}\right] \tilde{b}_{i_{1} i_{2}}^{(9)} .
$$

We note that, in order to arrive at Eq. (5.19), we have integrated over some directions of the loop momentum. The integration over the loop momentum is so simple because the projection on the transverse space is always given in terms of traceless tensors. To illustrate this point, consider a contribution of a general two-point function to the right-hand side of Eq. (5.1). It reads

$$
\begin{aligned}
& \int \frac{\mathrm{d}^{D} l}{(2 \pi)^{D}} \frac{1}{\left(l^{2}-m_{0}^{2}\right)\left(l^{2}+2 l \cdot q+q^{2}-m_{1}^{2}\right)}\left\{\tilde{b}_{0}+\tilde{b}_{1}\left(l \cdot n_{2}\right)\right. \\
& +\tilde{b}_{2}\left(l \cdot n_{3}\right)+\tilde{b}_{3}\left(l \cdot n_{4}\right)+\tilde{b}_{4}\left(\left(l \cdot n_{2}\right)^{2}-\left(l \cdot n_{4}\right)^{2}\right) \\
& +\tilde{b}_{5}\left(\left(l \cdot n_{3}\right)^{2}-\left(l \cdot n_{4}\right)^{2}\right)+\tilde{b}_{6}\left(l \cdot n_{2}\right)\left(l \cdot n_{3}\right)+\tilde{b}_{7}\left(l \cdot n_{3}\right)\left(l \cdot n_{4}\right) \\
& \left.+\tilde{b}_{8}\left(l \cdot n_{2}\right)\left(l \cdot n_{4}\right)+\tilde{b}_{9}\left(l \cdot n_{\epsilon}\right)^{2}\right\} .
\end{aligned}
$$

Because $q \cdot n_{\epsilon}=0, q \cdot n_{i}=0, i=2,3,4$, the integration over the directions of the transverse space $l_{\perp}=n_{2}\left(l \cdot n_{2}\right)+n_{3}\left(l \cdot n_{3}\right)+n_{4}\left(l \cdot n_{4}\right)+n_{\epsilon}\left(l \cdot n_{\epsilon}\right)$ is straightforward. We obtain

$$
\int \mathrm{d}^{D_{1}} l_{\perp} \delta\left(l_{\perp}^{2}-\mu_{0}^{2}\right)\left(l_{\perp}^{\mu}, l_{\perp}^{\mu} l_{\perp}^{\nu}\right)=\int \mathrm{d}^{D_{1}} l_{\perp} \delta\left(l_{\perp}^{2}-\mu_{0}^{2}\right)\left(0, \frac{g_{\perp}^{\mu \nu}}{D_{1}} l_{\perp}^{2}\right),
$$


where $D_{1}=D-1$.

Using this result in Eq. (5.21) together with the orthonormality property of the transverse space basis vectors $n_{i} n_{j}=\delta_{i j}$, we conclude that only two terms $\tilde{b}_{0}$ and $\tilde{b}_{9}$ contribute after the integration over the loop momentum is performed. The term with $\tilde{b}_{9}$ contributes to the rational part $\mathcal{R}$ in Eq. (5.19), while $\tilde{b}_{0}$ is the reduction coefficient of the relevant two-point master integral. Similar considerations apply to all other reduction coefficients, leading to Eq. (5.19). Clearly, we are interested in the calculation of quantities that are integrated over the loop momentum. It follows from Eq. (5.19) that, in addition to the rational part, we only require a modest number of the reduction coefficients $\tilde{e}^{(0)}, \tilde{d}^{(0)}, \tilde{c}^{(0)}, .$. etc. The question that we address now is how to find those coefficients efficiently.

In the course of the discussion of the two-dimensional case, we have seen that a powerful way to find coefficients $\tilde{e}_{i_{1} . . i_{5}}, \ldots, \tilde{a}_{i_{1}}$ involves calculations of both sides of Eq. (5.1) for special values of the loop momentum $l$, where a chosen subset of inverse Feynman propagators $d_{0}, d_{1}, \ldots, d_{N}$ vanish. We now discuss this procedure in detail, pointing out some subtleties that appear once we implement it.

We begin with the five-point function contribution. We choose five inverse propagators, say $d_{0}, d_{1}, \ldots d_{4}$ and find the loop momentum for which all of these inverse propagators vanish. This requires the momentum $l$ to span more than four dimensions, so, for definiteness, we make the minimal choice and take $l$ to be five-dimensional. Clearly, the only term in the right hand side of Eq. (5.1) that is non-zero is the term that does not contain any of the five propagators. This is $\tilde{e}_{01234}$ - the term that we would like to find. We argued previously that this term is constant, so computing the left hand side of Eq. (5.1) with the momentum $l^{*}$ such that $d_{0}\left(l^{*}\right)=0, d_{1}\left(l^{*}\right)=0, \ldots d_{4}\left(l^{*}\right)=0$, gives us $\tilde{e}_{01234}$.

While this procedure is correct, it often becomes impractical since it treats the scalar five-point function as a master integral. This would have been fully justified if we were interested in a five-dimensional calculation, but, in practical computations, we eventually take the limit $D \rightarrow 4$. In this limit, the five-point function becomes a linear combination of five four-point functions. We would therefore like to eliminate the five-point integral from the set of master integrals right away, avoiding large cancellations between four- and five-point functions in the $D \rightarrow 4$ limit. To see how this can be done note that the loop momentum in the five-point function can be written as

$$
l^{\mu}=\frac{1}{2} \sum_{i=1}^{4} v_{i}^{\mu}\left(d_{i}-d_{0}-\left(q_{i}^{2}-m_{i}^{2}+m_{0}^{2}\right)\right)+\left(l \cdot n_{\epsilon}\right) n_{\epsilon}^{\mu} .
$$

Squaring the two sides of this equation and using $l^{2}=d_{0}+m_{0}^{2}$, we see that for a loop momentum that satisfies $d_{0}=0, d_{1}=0, \ldots d_{4}=0$, we have

$$
\left(l \cdot n_{\epsilon}\right)^{2}=-\frac{1}{4} \sum_{i j}\left(v_{i} \cdot v_{j}\right)\left(q_{i}^{2}-m_{i}^{2}+m_{0}^{2}\right)\left(q_{j}^{2}-m_{j}^{2}+m_{0}^{2}\right)+m_{0}^{2} .
$$


It follows that we can either choose a scalar five-point function as the master integral or the integral with additional $\left(l \cdot n_{\epsilon}\right)^{2}$ in the numerator. However, because

$$
\lim _{D \rightarrow 4} \int \frac{\mathrm{d}^{D} l}{(2 \pi)^{D}} \frac{\left(l \cdot n_{\epsilon}\right)^{2}}{d_{0} d_{1} d_{2} d_{3} d_{4}} \rightarrow 0
$$

the second choice is preferable. Indeed, since the new master integral that we introduced to account for the need to employ dimensional regularization does not contribute in the $D \rightarrow 4$ limit, all four-dimensional relations between various integrals are automatically accounted for. Therefore this coefficient is only needed as a subtraction term in the determination of lower point coefficients. Experience shows that adopting this alternative definition of the pentagon coefficient leads to improved numerical stability in practical computations [67, 68].

To find the other coefficients, we follow the strategy already discussed in the context of two-dimensional computations. For example, having determined the five-point functions, we subtract their coefficients from the left-hand side of Eq. (5.1) and consider all the subsets of four propagators. We focus on one subset, $d_{0}, \ldots, d_{3}$ whose contribution is described by the coefficient

$$
\tilde{d}_{0123}=\tilde{d}_{0}+\tilde{d}_{1}\left(l \cdot n_{4}\right)+\tilde{d}_{2}\left(l \cdot n_{\epsilon}\right)^{2}+\tilde{d}_{3}\left(l \cdot n_{\epsilon}\right)^{2}\left(l \cdot n_{4}\right)+\tilde{d}_{4}\left(l \cdot n_{\epsilon}\right)^{4} .
$$

To determine $\tilde{d}_{0123}$, we find a momentum $l$ that satisfies $d_{0}(l)=0, d_{1}(l)=0$, $d_{2}(l)=0, d_{3}(l)=0$ and write it as

$$
\begin{aligned}
l^{\mu} & =V^{\mu}+l_{\perp}\left(\cos \phi n_{4}^{\mu}+\sin \phi n_{\epsilon}^{\mu}\right), \\
V^{\mu} & =-\frac{1}{2} \sum_{i}^{3} v_{i}^{\mu}\left(q_{i}^{2}-m_{i}^{2}+m_{0}^{2}\right),
\end{aligned}
$$

with $l_{\perp}=\sqrt{l_{\perp} \cdot l_{\perp}}$. It is sufficient to consider $l$ to be five-dimensional. The length of the projection of the vector $l$ on the transverse space is fixed

$$
l_{\perp}^{2}=m_{0}^{2}-V_{\mu} V^{\mu} .
$$

To find the $\tilde{d}_{0}, \ldots \tilde{d}_{3}$ coefficients, we take, for instance, $\sin \phi=0, \cos \phi= \pm 1$, denote $l_{ \pm}^{\mu}=V^{\mu} \pm l_{\perp} n_{4}^{\mu}$, calculate the numerator for these values of the loop momenta and find

$$
\tilde{d}_{0}=\frac{\operatorname{Num}\left(l_{+}\right)+\operatorname{Num}\left(l_{-}\right)}{2}, \quad \tilde{d}_{1}=\frac{\operatorname{Num}\left(l_{+}\right)-\operatorname{Num}\left(l_{-}\right)}{2 l_{\perp}} .
$$

To find $\tilde{d}_{2,3,4}$, we need to do a little bit more. First, we take $\cos \phi=\sin \phi=$ $\pm 1 / \sqrt{2}$, denote the loop momentum as $\tilde{l}_{ \pm}=V \pm l_{\perp}\left(n_{4}+n_{\epsilon}\right) / \sqrt{2}$, and find

$$
\begin{aligned}
& \tilde{d}_{2}+\tilde{d}_{4} \frac{l_{\perp}^{2}}{2}=l_{\perp}^{-2}\left(\operatorname{Num}\left(\tilde{l}_{+}\right)+\operatorname{Num}\left(\tilde{l}_{-}\right)-2 \tilde{d}_{0}\right), \\
& \tilde{d}_{3}=\sqrt{2} l_{\perp}^{-3}\left(\operatorname{Num}\left(\tilde{l}_{+}\right)-\operatorname{Num}\left(\tilde{l}_{-}\right)-\sqrt{2} \tilde{d}_{1} l_{\perp}\right) .
\end{aligned}
$$


We need yet another equation to resolve the $\tilde{d}_{2}-\tilde{d}_{4}$ degeneracy. It is convenient to take $l_{\epsilon}^{\mu}=V^{\mu}+l_{\perp} n_{\epsilon}^{\mu}$; this leads to

$$
\tilde{d}_{2}+\tilde{d}_{4} l_{\perp}^{2}=\frac{\operatorname{Num}\left(l_{\epsilon}\right)-\tilde{d}_{0}}{l_{\perp}^{2}} .
$$

We find

$$
\begin{aligned}
& \tilde{d}_{2}=\frac{1}{l_{\perp}^{2}}\left(2 \operatorname{Num}\left(\tilde{l}_{+}\right)+2 \operatorname{Num}\left(\tilde{l}_{-}\right)-\operatorname{Num}\left(l_{\epsilon}\right)-3 \tilde{d}_{0}\right), \\
& \tilde{d}_{4}=\frac{2}{l_{\perp}^{4}}\left(\operatorname{Num}\left(l_{\epsilon}\right)-\operatorname{Num}\left(\tilde{l}_{+}\right)-\operatorname{Num}\left(\tilde{l}_{-}\right)+\tilde{d}_{0}\right) .
\end{aligned}
$$

We next discuss how to compute the coefficients of the three-point functions. As an illustration, we choose a three point function with denominators $d_{0}, d_{1}, d_{2}$; its contribution is described by a coefficient

$$
\begin{aligned}
& \tilde{c}_{012}=\tilde{c}_{0}+\tilde{c}_{1}\left(l \cdot n_{3}\right)+\tilde{c}_{2}\left(l \cdot n_{4}\right)+\tilde{c}_{3}\left(\left(l \cdot n_{3}\right)^{2}-\left(l \cdot n_{4}\right)^{2}\right) \\
& +\tilde{c}_{4}\left(l \cdot n_{3}\right)\left(l \cdot n_{4}\right)+\tilde{c}_{5}\left(l \cdot n_{3}\right)^{3}+\tilde{c}_{6}\left(l \cdot n_{4}\right)^{3} \\
& +\tilde{c}_{7}\left(l \cdot n_{\epsilon}\right)^{2}+\tilde{c}_{8}\left(l \cdot n_{\epsilon}\right)^{2}\left(l \cdot n_{3}\right)+\tilde{c}_{9}\left(l \cdot n_{\epsilon}\right)^{2}\left(l \cdot n_{4}\right) .
\end{aligned}
$$

We choose the loop momentum that satisfies $d_{0}(l)=d_{1}(l)=d_{2}(l)=0$ and parametrize it as

$$
l^{\mu}=V^{\mu}+l_{\perp}\left(x_{3} n_{3}^{\mu}+x_{4} n_{4}^{\mu}+x_{\epsilon} n_{\epsilon}^{\mu}\right) .
$$

We consider momenta with $x_{\epsilon}=0$; such a choice allows us to determine the coefficients $\tilde{c}_{0, ., 6}$. If $x_{\epsilon}=0, x_{3}^{2}+x_{4}^{2}=1$, so that we can take $x_{3}=\cos \phi, x_{4}=$ $\sin \phi$. It is convenient then to rewrite Eq. (5.33) as a polynomial in $t=e^{i \phi}$. Eq. (5.33) becomes

$$
\tilde{c}_{012}(t)=\sum_{k=-3}^{3} c_{k} t^{k}
$$

where the coefficients $c_{k}$ read

$$
\begin{aligned}
& c_{ \pm 3}=\frac{\tilde{c}_{5} \pm i \tilde{c}_{6}}{8} l_{\perp}^{3}, \quad c_{ \pm 2}=\frac{2 \tilde{c}_{3} \mp i \tilde{c}_{4}}{4} l_{\perp}^{2}, \\
& c_{ \pm 1}=\left(\frac{1}{2} \tilde{c}_{1} \mp \frac{i}{2} \tilde{c}_{2}\right) l_{\perp}+\left(\frac{3}{8} \tilde{c}_{5} \mp \frac{3 i}{8} \tilde{c}_{6}\right) l_{\perp}^{3},
\end{aligned}
$$

and $c_{0}=\tilde{c}_{0}$. We can now use the technique of discrete Fourier transform, first discussed in the context of the OPP reduction, in Refs. [69, 70]. Application of the discrete Fourier transform allows us to write explicit expressions for the coefficients $c_{k}$ in a straightforward way. Indeed, they are given by

$$
c_{m}=\frac{1}{7} \sum_{n=0}^{6} \tilde{c}_{012}\left(t_{n}\right) t_{n}^{-m}
$$


where $t_{n}=e^{2 \pi i n / 7}$. To prove this equation, note that

$$
\sum_{n=0}^{k} e^{\frac{2 \pi i n}{k+1} r}=\delta_{r 0}(k+1)
$$

Substituting Eq. (5.35) into the right hand side of Eq. (5.37) and carrying out the summation over $n$ using Eq. (5.38), we can easily show that the right hand side of Eq. (5.37) is indeed equal to one of the $c$-coefficients. Eq. (5.37) provides a convenient way to find the cut-constructible coefficients of the three-point function. Finally, to determine the rational part coefficients in Eq. (5.33), we take vectors $l$ that have non-vanishing projections on either $n_{3}$ and $n_{\epsilon}$ or on $n_{4}$ and $n_{\epsilon}$. Since we already know all the cut-constructible coefficients, it is straightforward to find $\tilde{c}_{7,8,9}$.

We note that the discrete Fourier transform is just one of many ways to solve the linear system of equations required to obtain the coefficients $\tilde{c}_{0}, \ldots \tilde{c}_{6}$. It is a convenient, easy-to-code-up procedure, but it is neither unique nor superior to other ways. In fact, it is clear that in certain cases it is better to avoid using the discrete Fourier transform method and to solve the system of equations by other means.

To see why this might be the case, we discuss the computation of the reduction coefficients for the two-point function with two propagators $d_{0}$ and $d_{1}$. Then, the physical space is one-dimensional and the transverse space is threedimensional. The momentum parametrization therefore reads

$$
l^{\mu}=x_{1} q_{1}^{\mu}+l_{\perp}\left(\sum_{i=2}^{4} x_{i} n_{i}^{\mu}+x_{\epsilon} n_{\epsilon}^{\mu}\right), \quad q_{1} \cdot n_{i \geq 2}=0, \quad n_{i} \cdot n_{j}=\delta_{i j} .
$$

Using Eq. (5.39), we find that components of the momentum $l$ for which $d_{0,1}=0$ are subject to the following constraints

$$
x_{1}=\frac{\left(m_{1}^{2}-m_{0}^{2}-q_{1}^{2}\right)}{2 q_{1}^{2}}, \quad l_{\perp}^{2}=m_{0}^{2}-x_{1}^{2} q_{1}^{2}, \quad x_{2}^{2}+x_{3}^{2}+x_{4}^{2}+x_{\epsilon}^{2}=1 .
$$

The general parametrization of the $\tilde{b}$-coefficient reads

$$
\begin{aligned}
\tilde{b}_{01} & =\tilde{b}_{0}+\tilde{b}_{1}\left(l \cdot n_{2}\right)+\tilde{b}_{2}\left(l \cdot n_{3}\right)+\tilde{b}_{3}\left(l \cdot n_{4}\right) \\
& +\tilde{b}_{4}\left(\left(l \cdot n_{2}\right)^{2}-\left(l \cdot n_{4}\right)^{2}\right)+\tilde{b}_{5}\left(\left(l \cdot n_{3}\right)^{2}-\left(l \cdot n_{4}\right)^{2}\right)+\tilde{b}_{6}\left(l \cdot n_{2}\right)\left(l \cdot n_{3}\right) \\
& +\tilde{b}_{7}\left(l \cdot n_{3}\right)\left(l \cdot n_{4}\right)+\tilde{b}_{8}\left(l \cdot n_{2}\right)\left(l \cdot n_{4}\right)+\tilde{b}_{9}\left(l \cdot n_{\epsilon}\right)^{2}
\end{aligned}
$$

Similar to the case of the three-point function, there are infinitely many loop momenta that satisfy the constraints shown in Eq. (5.40). Therefore, to find the cut-constructible coefficients, we can proceed as before, parameterizing

$$
l_{\perp}^{\mu}=l_{\perp}\left(\sin \theta \cos \phi n_{2}^{\mu}+\sin \theta \sin \phi n_{3}^{\mu}+\cos \theta n_{4}^{\mu}\right),
$$

and then applying the technique of the discrete Fourier transform to determine $\tilde{b}_{0}, \ldots \tilde{b}_{8}$. Note, however, that the application of the discrete Fourier transform 
requires division by $l_{\perp}$, c.f. Eq. (5.36), and this may lead to potential trouble. Indeed, according to Eq. (5.40), $l_{\perp}$ vanishes if $m_{0}^{2}=x_{1}^{2} q_{1}^{2}$ which corresponds to $q_{1}^{2}=\left(m_{0}-m_{1}\right)^{2}$ or $q_{1}^{2}=\left(m_{0}+m_{1}\right)^{2}$. These kinematic points are not dangerous if only massless virtual particles are considered. However, the situation may become problematic if virtual massive particles are present in the calculation. Note also that close to those exceptional values of $q_{1}^{2}, l_{\perp}$ can be small, so that division by $l_{\perp}$ may lead to numerical instabilities.

To handle the case of small $l_{\perp}$ in a numerically stable way, the method of discrete Fourier transform is not directly applicable and the system of equations must be solved differently. There are many ways to solve a system of linear equations avoiding division by $l_{\perp}$; one option is described below. We begin by choosing $l_{\perp}^{ \pm}=x_{\perp} n_{2} \pm x_{3} n_{3}, l_{\perp}^{ \pm} \cdot l_{\perp}^{ \pm}=l_{\perp}^{2}$. Recall that $l_{\perp}^{2}$ is fixed by the on-shell condition Eq. (5.40) and therefore $x_{3}$ is expressed through $x_{\perp}, x_{3}=\sqrt{l_{\perp}^{2}-x_{\perp}^{2}}$. We calculate $b_{ \pm}=b\left(l^{ \pm}\right)$and eliminate $x_{3}^{2}$ in favor of $l_{\perp}^{2}$ and $x_{\perp}$ where possible. We obtain

$$
b_{ \pm}=\tilde{b}_{0}+\tilde{b}_{1} x_{\perp} \pm x_{3} \tilde{b}_{2}+\tilde{b}_{4} x_{\perp}^{2}+\tilde{b}_{5} x_{3}^{2} \pm \tilde{b}_{6} x_{\perp} x_{3} .
$$

Taking the sum and the difference of $b_{ \pm}$, we arrive at

$$
\frac{\left(b_{+}+b_{-}\right)}{2}=\tilde{b}_{0}^{\mathrm{eff}}+\tilde{b}_{1} x_{\perp}+\tilde{b}_{4}^{\mathrm{eff}} x_{\perp}^{2}, \quad \frac{\left(b_{+}-b_{-}\right)}{2 x_{3}}=\tilde{b}_{2}+\tilde{b}_{6} x_{\perp},
$$

where

$$
\tilde{b}_{0}^{\mathrm{eff}}=\tilde{b}_{0}+\tilde{b}_{5} l_{\perp}^{2}, \quad \tilde{b}_{4}^{\mathrm{eff}}=\tilde{b}_{4}-\tilde{b}_{5} .
$$

The right hand sides of these equations are polynomials in $x_{\perp}$. Therefore, we can apply a discrete Fourier transform with respect to $x_{\perp}$ to find coefficients $\tilde{b}_{1}, \tilde{b}_{4}^{\text {eff }}, \tilde{b}_{0}^{\text {eff }}$ as well as $\tilde{b}_{2}, \tilde{b}_{6}$ in Eq. (5.44).

To determine the remaining coefficients, we make five choices of the loopmomentum, satisfying the on-shell condition. We choose for instance

$$
\begin{aligned}
& l^{(a)}=x_{1} q_{1}+x n_{2}+y n_{4}, \\
& l^{(b)}=x_{1} q_{1}-x n_{2}+y n_{4}, \\
& l^{(c)}=x_{1} q_{1}-x n_{2}-y n_{4}, \\
& l^{(d)}=x_{1} q_{1}+x n_{4}+y n_{3}, \\
& l^{(e)}=x_{1} q_{1}+x n_{2}+y n_{\epsilon},
\end{aligned}
$$

where $x^{2}+y^{2}=l_{\perp}^{2}$. We use the notation $b_{\alpha}=b\left(l^{(\alpha)}\right)$. With the coefficients $\tilde{b}_{0}^{\text {eff }}, \tilde{b}_{1}, \tilde{b}_{2}, \tilde{b}_{4}^{\text {eff }}$ and $\tilde{b}_{6}$ in hand, we determine the other coefficients in the sequence, 
$\tilde{b}_{8}, \tilde{b}_{3}, \tilde{b}_{5}, \tilde{b}_{7}, \tilde{b}_{9}, \tilde{b}_{0}, \tilde{b}_{4}$. The results are

$$
\begin{aligned}
& \tilde{b}_{8}=\frac{\left(\frac{1}{2}\left(b_{a}-b_{b}\right)-x \tilde{b}_{1}\right)}{x y}, \\
& \tilde{b}_{3}=\frac{\frac{1}{2}\left(b_{a}-b_{c}\right)-\tilde{b}_{1} x}{y}, \\
& \tilde{b}_{5}=\frac{\tilde{b}_{0}^{\text {eff }}+\tilde{b}_{3} y+y x \tilde{b}_{8}+x \tilde{b}_{1}+\left(x^{2}-y^{2}\right) \tilde{b}_{4}^{\text {eff }}-b_{a}}{3 y^{2}}, \\
& \tilde{b}_{7}=\frac{\left(b_{d}-y^{2} \tilde{b}_{5}+\tilde{b}_{5} x^{2}+\tilde{b}_{4} x^{2}-\tilde{b}_{3} x-y \tilde{b}_{2}-\tilde{b}_{0}\right)}{x y}, \\
& \tilde{b}_{9}=\frac{\left(b_{e}-\tilde{b}_{4} x^{2}-\tilde{b}_{1} x-\tilde{b}_{0}\right)}{y^{2}} .
\end{aligned}
$$

The coefficients $\tilde{b}_{0}$ and $\tilde{b}_{4}$ are determined using Eq. (5.45) once $\tilde{b}_{5}$ has been fixed.

We have discussed a method to calculate the coefficients $\tilde{b}_{1, \ldots, 9}$ in a numerically stable way for small values of $l_{\perp}$. Note that we used the fact that even for arbitrarily small $l_{\perp}^{2}$ we can choose large, complex values of $x, y$ with $x^{2}+y^{2}=l_{\perp}^{2}$. In a numerical program, one can switch from the discrete Fourier transform to the solution just described, depending on the value of $l_{\perp}$. However, the proposed methods can only work if the decomposition of the loop momentum, as in Eq. (5.39), exists. A glance at Eq. (5.40) makes it clear that the decomposition fails for the light-like momentum, $q_{1}^{2}=0$, and we have to handle this case differently. We describe a possible solution below.

First, some clarifications are in order. Because we are interested in oneloop calculations for infrared safe observables, it is reasonable to assume that the vector $q_{1}$ can be exactly light-like but it is impossible for that vector to be nearly light-like, since such kinematic configurations are, typically, rejected by cuts $^{5}$. Hence, we have to modify the above analysis to allow for an exactly light-like external momentum. To this end, we choose a frame where the fourvector in Eq. (5.39) reads $q_{1}=(E, 0,0, E)$. We introduce a complementary light-like vector $\bar{q}_{1}=(E, 0,0,-E)$. The loop momentum is parametrized as $l=x_{1} q_{1}+x_{2} \bar{q}_{1}+l_{\perp}$. We denote the basis vectors of the transverse space as $n_{3,4}$; they satisfy $n_{i} \cdot n_{j}=\delta_{i j}, q_{1} \cdot n_{3,4}=0, \bar{q}_{1} \cdot n_{3,4}=0$. The on-shell condition for the loop momentum fixes $x_{2}$

$$
x_{2}=\frac{m_{1}^{2}-m_{0}^{2}}{s}, \quad s=2 q_{1} \cdot \bar{q}_{1},
$$

and a linear combination of $x_{1}$ and $l_{\perp}^{2}$

$$
l_{\perp}^{2}+m_{1}^{2} x_{1}-m_{0}^{2}\left(1+x_{1}\right)=0 .
$$

\footnotetext{
${ }^{5}$ External particles with small masses are obvious exceptions but rarely do we need to know observables for, say, massive $b$-quarks in a situation when all kinematic invariants are large.
} 
Compared to the case when the reference vector $q_{1}$ is not on the light-cone, we write now the parametrization of the function $\tilde{b}$ using $n_{4} \cdot l$. We choose it to be

$$
\begin{aligned}
\tilde{b}(l)= & \tilde{b}_{0}+\tilde{b}_{1}\left(\bar{q}_{1} \cdot l\right)+\tilde{b}_{2}\left(n_{3} \cdot l\right)+\tilde{b}_{3}\left(n_{4} \cdot l\right)+\tilde{b}_{4}\left(\bar{q}_{1} \cdot l\right)\left(\bar{q}_{1} \cdot l\right) \\
& +\tilde{b}_{5}\left(\bar{q}_{1} \cdot l\right)\left(n_{3} l\right)+\tilde{b}_{6}\left(\bar{q}_{1} \cdot l\right)\left(n_{4} \cdot l\right)+\tilde{b}_{7}\left(\left(n_{3} \cdot l\right)^{2}-\left(n_{4} \cdot l\right)^{2}\right) \\
& +\tilde{b}_{8}\left(n_{3} \cdot l\right)\left(n_{4} \cdot l\right)+\tilde{b}_{9}\left(l \cdot n_{\epsilon}\right)^{2} .
\end{aligned}
$$

We describe a procedure to find the coefficients $\tilde{b}_{0}, \ldots \tilde{b}_{9}$ in a numerically stable way. We begin by choosing $x_{1}=0.5$ and this choice is not particularly well-motivated. This fixes $l_{\perp}^{2}$, and $x_{2}$ is fixed by the on-shell condition Eq. (5.48). The freedom remains to choose the direction of the vector $l_{\perp}$ in the $\left(n_{3}, n_{4}\right)$ plane. Consider four different vectors

$$
l_{\perp}^{(a)}=y n_{3}+x n_{4}, l_{\perp}^{(b)}=-y n_{3}+x n_{4}, l_{\perp}^{(c)}=y n_{3}-x n_{4}, l_{\perp}^{(d)}=-y n_{3}-x n_{4},
$$

where $x^{2}+y^{2}=l_{\perp}^{2}$. We use vectors $l^{(\alpha)}=x_{1} q_{1}+x_{2} \bar{q}_{1}+l_{\perp}^{(\alpha)}, \alpha=a, b, c, d$, to calculate the function $b^{(\alpha)}=\tilde{b}\left(l^{\alpha}\right)$. Using $b_{a}, \ldots b_{d}$, we can immediately find the coefficient $\tilde{b}_{8}$

$$
\tilde{b}_{8}=\frac{1}{4 x y}\left(b^{(a)}-b^{(c)}-b^{(b)}+b^{(d)}\right) .
$$

For the determination of the remaining coefficients, it is convenient to introduce two linear combinations

$$
\begin{aligned}
& b_{36}=\frac{1}{4 x}\left(b^{(a)}-b^{(c)}+b^{(b)}-b^{(d)}\right), \\
& b_{25}=\frac{1}{2}\left(b^{(a)}-b^{(b)}-2 x y b_{8}\right) .
\end{aligned}
$$

As the next step, we choose $x_{1}=-0.5$. Note that this changes the value of $l_{\perp}^{2}$ according to Eq. (5.49). We then repeat the calculation described above. Our choices of momenta in the transverse plane $l_{\perp}$ are the same as in Eq. (5.51) but, to avoid confusion, we emphasize that $x$ and $y$ have to be calculated with the new $l_{\perp}^{2}$. We will refer to $b$ computed with those new vectors as $\bar{b}^{(a)}, \bar{b}^{(b)}$, etc. We calculate $\bar{b}_{36,25}$ by substituting $b^{(\alpha)} \rightarrow \bar{b}^{\alpha}$ in Eq. (5.53). It is easy to see that simple linear combinations give the desired coefficients

$$
\begin{aligned}
\tilde{b}_{3}=\frac{1}{2}\left(b_{36}+\bar{b}_{36}\right), & \tilde{b}_{6}=\frac{2}{s}\left(b_{36}-\bar{b}_{36}\right), \\
\tilde{b}_{2}=\frac{1}{2}\left(b_{25}+\bar{b}_{25}\right), & \tilde{b}_{5}=\frac{2}{s}\left(b_{25}-\bar{b}_{25}\right) .
\end{aligned}
$$

Other coefficients, required for the complete parametrization of the function $\tilde{b}(l)$ in Eq. (5.50), are obtained along similar lines; we do not discuss this further. However, we emphasize that the procedure that we just described is important for the computation of one-loop virtual amplitudes in a situation where both massless and massive particles are involved. In particular, it is heavily used in computations of NLO QCD corrections to top quark pair production discussed in Refs. [71, 72]. 
As a final remark, we note that there is another important difference between reducing the two-point function to scalar integrals for a light-like and a nonlight-like vector. Consider only cut-constructible terms. Then, for $q_{1}^{2} \neq 0$ the integration over the transverse space can be immediately done, leading to

$$
\int \frac{\mathrm{d}^{D} l}{(2 \pi)^{D}} \frac{\tilde{b}(l)}{d_{0} d_{1}}=\tilde{b}_{0} \int \frac{\mathrm{d}^{D} l}{(2 \pi)^{D}} \frac{1}{d_{0} d_{1}} .
$$

Hence, the only integral we need to know in $q_{1}^{2} \neq 0$ case is the scalar two-point function. However, in case of a light-like vector $q_{1}^{2}=0$, three master integrals contribute to the cut-constructible part even after averaging over the directions of the vector $l$ in the (two-dimensional) transverse space

$$
\int \frac{\mathrm{d}^{D} l}{(2 \pi)^{D}} \frac{\tilde{b}(l)}{d_{0} d_{1}}=\int \frac{\mathrm{d}^{D} l}{(2 \pi)^{D}} \frac{\tilde{b}_{0}+\tilde{b}_{1}\left(\bar{q}_{1} \cdot l\right)+\tilde{b}_{4}\left(\bar{q}_{1} \cdot l\right)^{2}}{d_{0} d_{1}} .
$$

Those integrals must be included in the basis of master integrals in the case when double cuts are considered with a light-like external vector. The calculation of those additional master integrals is straightforward. For the sake of example, we give the results below for the equal mass case $m_{0}=m_{1}=m$. We introduce $d_{0}=l^{2}-m^{2}, d_{1}=\left(l+q_{1}\right)^{2}-m^{2}, q_{1}^{2}=0, \bar{q}_{1} \cdot q_{1}=r, c_{\Gamma}=(4 \pi)^{\epsilon-2} \Gamma(1+\epsilon) \Gamma(1-$ $\epsilon)^{2} / \Gamma(1-2 \epsilon)$ and find $(D=4-2 \epsilon)$

$$
\begin{aligned}
\frac{\mu^{2 \epsilon}}{\mathrm{i} r_{\Gamma}} \int \frac{\mathrm{d}^{D} l}{(2 \pi)^{D}} \frac{l \cdot \bar{q}_{1}}{d_{0} d_{1}} & =-\frac{r}{2}\left(\frac{1}{\epsilon}+\ln \left(\frac{\mu^{2}}{m^{2}}\right)\right), \\
\frac{\mu^{2 \epsilon}}{\mathrm{i} r_{\Gamma}} \int \frac{\mathrm{d}^{D} l}{(2 \pi)^{D}} \frac{\left(l \cdot \bar{q}_{1}\right)\left(l \cdot \bar{q}_{1}\right)}{d_{0} d_{1}} & =\frac{r^{2}}{3}\left(\frac{1}{\epsilon}+\ln \left(\frac{\mu^{2}}{m^{2}}\right)\right) .
\end{aligned}
$$

\subsection{Comments on the rational part}

The most general parameterizations of $\tilde{e}, \tilde{d}, \tilde{c}, \tilde{b}$ and $\tilde{a}$-functions contain two types of terms. First, there are terms that involve scalar products of the loop momenta with four-dimensional vectors from various transverse spaces. Second, there are terms that involve scalar products of the loop momentum with the $(D-4)$-dimensional components of the vectors spanning the transverse space. These latter terms require going beyond the four-dimensional loop momentum and give rise to the rational part.

The rational part is related to the ultraviolet behavior of the theory; the naive expectation is that the better the UV behavior, the "smaller" the rational

part. If the integral is free from the rational part and, therefore, can be fully obtained by considering loop momenta confined to the four-dimensional space, it is said to be "cut-constructible". A natural expectation is that the rational part is absent in UV-finite integrals. As we explain below, this expectation turns out to be wrong; the correct result is that a Feynman $N$-point integral is cut constructible, provided that tensor rank, $r$, of the integral satisfies the following condition [73]

$$
r<\max \{(N-1), 2\} .
$$




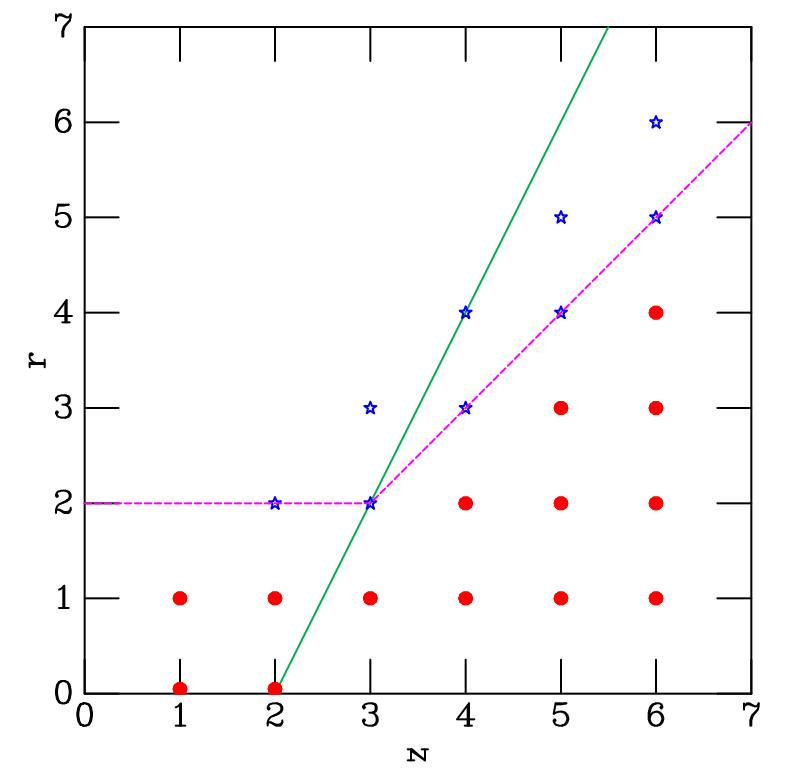

Figure 5.1: Diagram showing tensor $N$-point integrals of rank $r$. Integrals shown by bullets (red) are cut-constructible, integrals denoted by stars (blue) contain rational terms. The UV finite integrals lie beneath the solid (green) line, whereas the cut constructible integrals lie beneath the dashed (purple) line.

The condition is illustrated in Fig. 5.1. If it is violated the integral will contain rational parts. Explicitly, Eq. (5.59) implies that the UV finite ranktwo four-point function is cut-constructible, whereas the UV-finite rank-three four-point function is not.

To prove this assertion, we will consider all the integrals that occur in a renormalizable theory case by case. To this end, consider a class of higher-rank three-point functions that are present in renormalizable quantum field theories; they include rank-one, rank-two and rank-three tensor three-point functions. The rank-one three-point function is cut-constructible since its reduction to scalar integrals can not contain terms with $\left(l \cdot n_{\epsilon}\right)$ tensor structure. Following the discussion at the beginning of this Section, it is easy to see that reduction of a general rank-two three-point function contains an integral

$$
\int \frac{\mathrm{d}^{D} l}{(2 \pi)^{D}} \frac{\left(l \cdot n_{\epsilon}\right)^{2}}{d_{0} d_{1} d_{2}} \neq 0 \text { in the limit } \epsilon \rightarrow 0 .
$$

Hence, the reduction of a rank-two three-point function to scalar integrals contains the remnant of the analytic continuation of the loop momentum to $D$ dimensions - a sign that the rank-two three-point function has a rational part. Because the reduction of a rank-three three-point function immediately leads to either rank-two three-point functions or rank-two two-point functions, we conclude that the reduction of a rank-three three-point function contains a rational 
part as well. Note that while the rational part is a remnant of UV-sensitivity of a Feynman integral, it is a finite contribution to the final answer; all UVdivergent contributions reside in scalar two- and one-point scalar integrals and are, in fact, cut-constructible.

It is now easy to generalize the above arguments to higher-point functions. For example, the rank-one four-point function is clearly cut-constructible. The rank-two four-point functions are also cut-constructible but in a slightly more subtle manner. Indeed, the rank-two four-point functions are reduced to rankone four-point functions and rank-one three-point functions, both of which are cut-constructible, and to a rank-two four-point integral of the type

$$
\int \frac{\mathrm{d}^{D} l}{(2 \pi)^{D}} \frac{\left(l \cdot n_{\epsilon}\right)^{2}}{d_{0} d_{1} d_{2} d_{3}} .
$$

It is not difficult to see that this integral can be disregarded in the $\epsilon \rightarrow 0$ limit and this is the reason why the rank-two four-point function does not have a rational part. Finally, since the reduction of a rank-three four-point function leads to a host of rank-two three-point functions, we conclude that the rankthree four-point function is not cut-constructible. The results for the rational parts of certain tensor integrals are given in Appendix B.

Finally, we comment on the computation of the rational part advocated in Ref. [74]. The idea is to split the numerator function of any Feynman diagram into "four-dimensional" terms and $(D-4)$-dimensional terms. The calculation of the rational part, related to first class of terms $-\mathcal{R}_{1}-$ is complicated and requires full machinery behind the OPP technology, that we discussed in this Section. One can think of $\mathcal{R}_{1}$ as the rational part that appears in ultraviolet-finite, pure four-dimensional integrals as a consequence of the tensor reduction. On the contrary, the rational part $\mathcal{R}_{2}$ is associated with explicit $(D-4)$-dimensional terms in Feynman diagrams and, for this reason, its calculation is easy. Indeed, consider a Feynman diagram with a numerator $\operatorname{Num}(l)$ that depends on the loop momentum $l=\left(l_{4}, l_{\epsilon}\right)$, where $l_{4}$ is the four-dimensional part of the loop momentum and $l_{\epsilon}$ is its $(D-4)$-dimensional part. We write the numerator as

$$
\operatorname{Num}(l)=\operatorname{Num}_{4}\left(l_{4}\right)+\operatorname{Num}_{\epsilon}\left(l_{\epsilon}, \epsilon\right) .
$$

The rational part $\mathcal{R}_{2}$ by definition comes from the second term in the above equation. We now explain why it is easy to compute $\mathcal{R}_{2}$. First note, that the exact form of $\mathcal{R}_{2}$ depends on the regularization scheme. For example, in the four-dimensional helicity scheme [75], explicit $\epsilon$-terms in Eq. (5.62) are not needed. Second, since momenta and polarization vectors of all external particles in Eq. (5.62) are kept four-dimensional, $\mathrm{Num}_{\epsilon}$ in Eq. (5.62) can only depend on $l_{\epsilon}^{2}$. As such, it can only contain terms with the loop momentum squared. Third, if a particular diagram is ultraviolet-finite, we can neglect $\operatorname{Num}_{\epsilon}\left(l_{\epsilon}, \epsilon\right)$ in Eq. (5.62), so that the particular diagram does not contribute to $R_{2}$. We conclude that $\mathcal{R}_{2}$ only comes from divergent graphs, in sharp contrast to $\mathcal{R}_{1}$. Since there is a small number of divergent one-loop graphs in any renormalizable theory, the computation of the rational part $\mathcal{R}_{2}$ becomes straightforward. Note 
that because the ultraviolet sensitivity of a particular diagram depends on the gauge-fixing condition, results for $\mathcal{R}_{2}$ are, in general, gauge-dependent.

To illustrate a typical calculation of $\mathcal{R}_{2}$, we compute it in QED. We begin by choosing the Landau gauge. In this gauge, there is one divergent diagram in QED at the one-loop order - the fermion loop contribution to photon vacuum polarization diagram. The $\mathcal{R}_{2}$ for QED is then easily computed

$$
\begin{gathered}
\mathcal{R}_{2}\left[\Pi_{\mu \nu}\right]=\mathcal{R}_{2}\left[i e^{2} \int \frac{\mathrm{d}^{D} l}{(2 \pi)^{D}} \frac{\operatorname{Tr}\left[\gamma_{\mu}(\hat{l}+m) \gamma_{\nu}(\hat{l}+\hat{q}+m)\right]}{d_{0} d_{1}}\right] \\
=\mathcal{R}_{2}\left[i e^{2} \int \frac{\mathrm{d}^{D} l}{(2 \pi)^{D}} \frac{\operatorname{Tr}\left[\gamma_{\mu} \hat{l} \gamma_{\nu} \hat{l}\right]}{d_{0} d_{1}}\right]=-4 i e^{2} g_{\mu \nu} \int \frac{\mathrm{d}^{D} l}{(2 \pi)^{D}} \frac{\left(l \cdot n_{\epsilon}\right)^{2}}{d_{0} d_{1}} \\
=-g_{\mu \nu} \frac{\alpha}{2 \pi}\left(2 m^{2}-\frac{q^{2}}{3}\right) .
\end{gathered}
$$

If we switch to the Feynman gauge, the above result stays the same but two additional divergent diagrams appear - the one-loop correction to the photonelectron interaction vertex and the one-loop correction to the fermion self-energy diagram. In the four-dimensional helicity scheme, the fermion self-energy diagram does not contribute to $\mathcal{R}_{2}$, since the rank of the tensor integrals present there is not high enough. The contribution of the one-loop photon-fermion vertex correction to $\mathcal{R}_{2}$ in the four-dimensional helicity scheme reads

$$
\mathcal{R}_{2}\left[V_{\mu}\right]=-i e \gamma_{\mu} \int \frac{\mathrm{d}^{D} l}{(2 \pi)^{D}} \frac{\left(-4 i e^{2}\right)\left(l \cdot n_{\epsilon}\right)^{2}}{d_{0} d_{1} d_{2}}=-i e \gamma_{\mu} \times \frac{\alpha}{2 \pi} .
$$

The explicit results for the rational part $\mathcal{R}_{2}$ in various theories, including QCD and the Standard Model were recently given in Refs. [76, 77, 78]. Finally we note that yet another unitarity-based method for the calculation of the rational part has been developed $[79,80]$. It is based on the recursion relations for on-shell matrix elements.

\subsection{Rational terms by Passarino-Veltman reduction}

In this Section we give an alternative proof of the condition that an integral has to satisfy for being cut-constructible, Eq. (5.59). This proof is based on the Passarino-Veltman reduction. We will proceed case-by-case for the two-, threeand four-point integrals which occur in a renormalizable theory. The extension to higher-point integrals will be performed at the end. We first note that the Passarino-Veltman decomposition described in Section 2 and Appendix A, yields the coefficients of the scalar integrals $D_{0}, C_{0}, B_{0}, A_{0}$ for arbitrary values of the number of dimensions. Since the rational terms are related to ultraviolet singularities they will show up at the end of the reduction as terms of the form

$$
\text { Rational terms } \sim \epsilon B_{0}\left(p, m_{i}, m_{j}\right), \epsilon A_{0}\left(m_{i}\right),
$$


because $A_{0}$ and $B_{0}$ are the only ultraviolet-divergent scalar integrals. Such terms can only arise if the reduction involves the dimensional parameter $D$. This means that integrals of rank $r$ less than two will always be cut-constructible, since their reduction coefficients are $D$-independent. On the contrary, ultravioletdivergent integrals of rank two or greater (e.g. $D_{i i i i}, C_{i i i}, C_{i i}, B_{i i}$ ) will give rise to rational parts. Thus it only remains to discuss the ultraviolet finite integrals $D_{i i i}, D_{i i}$. The integral $D_{i i i}$ contains a ultraviolet-divergent integrals of rank greater than two in its reduction paths, $D_{i i i} \rightarrow C_{i i}$, see Table 2.1 , and hence it will have a rational part. This leaves the special case $D_{i i}$, a finite integral which can contain a ultraviolet-divergent integral in its reduction path, namely $B_{0}$. However since the starting integral is ultraviolet finite, the ultraviolet poles all cancel. Moreover the coefficients of $B_{0}$ are all $\epsilon$-independent, since the only $D$ dependence enters through $D_{00}$, which does not contain $B_{0}$ in its reduction path. Hence the rank-two, four-point integral is cut constructible.

In a renormalizable theory the higher-point functions are not ultravioletdivergent. Moreover the most ultraviolet-singular terms in their reduction paths reduce both $N$ and $r$ by one unit. Therefore the reduction paths of these ultraviolet-finite integrals can only generate a rational part if the rank of the integral has $r \geq N-1$. This observation extends Eq. (5.59) to $N$ greater than four.

\section{Managing the color}

In this Section, we describe how to connect the OPP ideas, discussed in the previous Section in the context of individual Feynman diagrams, with unitarity ideas. The object that we need to calculate is the one-loop scattering amplitude $\mathcal{A}(\{k\},\{\epsilon\},\{a\})$, where external particles of definite types have on-shell momenta $\{k\}$, polarizations $\{\epsilon\}$ and color indices $\{a\}$. Before discussing unitarity ideas, we explain how color degrees of freedom can be treated, in order to simplify the computation of scattering amplitudes.

Any Feynman diagram that contributes to a scattering amplitude of colored particles involves a color part and a space-time part. Since the presence of color degrees of freedom causes some additional complexity in the evaluation of scattering amplitudes, it is important to simplify the treatment of color as much as possible. To deal with color parts of the amplitude in a systematic manner, it is customary to choose a basis in color space and express the color parts of all diagrams as linear combinations of the basis elements [28, 81]. This procedure is known as the "color decomposition". A pedagogical description of the color decomposition can be found in Refs. [82, 83]. In what follows we present a simple but concise discussion of the color decomposition, using basisindependent features to the extent possible.

\section{1. n-gluon amplitudes}

As a first step, we discuss the color decomposition of $n$-gluon tree amplitudes in $S U\left(N_{c}\right)$ gauge theories. The scattering amplitude $\mathcal{A}_{n}^{\text {tree }}$ depends on the gluon 
color quantum numbers $a_{i}=1, \ldots,\left(N_{c}^{2}-1\right)$, the helicities $h_{i}= \pm 1$ and the momenta $k_{i}$. We assume that the momenta of all gluons are outgoing, so that momentum conservation reads $k_{1}+k_{2} \ldots+k_{n}=0$. In the remainder of this Section, we suppress helicity and momentum labels when writing scattering amplitudes.

The generators of the Lie algebra of the $S U\left(N_{c}\right)$ gauge group in the fundamental representation are determined by the following sets of equations

$$
\left[T^{a}, T^{b}\right]=i \sqrt{2} f^{a b c} T^{c}=-F_{b c}^{a} T^{c}, \quad \operatorname{Tr}\left(T^{a} T^{b}\right)=\delta_{a b} .
$$

The color factors of the three- and four-gluon vertices are given by the structure constants $f^{a b c}$ of the $S U\left(N_{c}\right)$ group. It is well-known that structure constants can be used to define Lie algebra generators in the adjoint representation

$$
\left[F^{a}, F^{b}\right]=-F_{b c}^{a} F^{c}, \quad F_{b c}^{a}=-i \sqrt{2} f^{a b c}, \quad \operatorname{Tr}\left(F^{a} F^{b}\right)=2 N_{c} \delta_{a b} .
$$

The first equation in Eq. (6.2) follows from the Jacobi identity and the commutator algebra of fundamental representation, Eq. (6.1). Various normalization factors are chosen for convenience. We therefore conclude that the color part of any contributing Feynman diagram is a product of $(n-2)$ color matrices in the adjoint representation

$$
\left(F^{a_{2}} F^{a_{3}} \ldots F^{a_{n-2}} F^{a_{n-1}}\right)_{a_{1} a_{n}},
$$

and, possibly, some other terms obtained by permutations of the color indices of the gluons.

It follows from Eqs. $(6.1,6.2)$ that the adjoint color matrix $F^{a}$ can be specified also as the trace of three color matrices of fundamental - and in fact any representation. We obtain

$$
\left(F^{a_{1}}\right)_{a_{2} a_{3}}=-\frac{1}{2 N_{c}} \operatorname{Tr}\left(\left[F^{a_{1}}, F^{a_{2}}\right] F^{a_{3}}\right)=-\operatorname{Tr}\left(\left[T^{a_{1}}, T^{a_{2}}\right] T^{a_{3}}\right) .
$$

We can use Eq. (6.4), to convert the product of color matrices in the adjoint representation into linear combinations of traces of strings of $F$ - and $T$-matrices

$$
\begin{aligned}
\left.\left(F^{a_{2}} F^{a_{3}} \ldots F^{a_{(n-2)}} F^{a_{(n-1)}}\right)\right)_{a_{1} a_{n}} & \\
& =\frac{1}{2 N_{c}} \operatorname{Tr}\left(\left[\left[\ldots\left[\left[F^{a_{1}}, F^{a_{2}}\right], F^{a_{3}}\right], \ldots, F^{a_{n-2}}\right]\left[F^{a_{n-1}}, F^{a_{n}}\right]\right)\right. \\
& =\operatorname{Tr}\left(\left[\left[\ldots\left[\left[T^{a_{1}}, T^{a_{2}}\right], T^{a_{3}}\right], \ldots, T^{a_{n-2}}\right]\left[T^{a_{n-1}}, T^{a_{n}}\right]\right) .\right.
\end{aligned}
$$

For example, Eq. (6.5) gives the following $F$-matrix identities for $n=4$ and 5,

$$
\begin{aligned}
& \left(F^{a_{2}} F^{a_{3}}\right)_{a_{1} a_{4}}=\frac{1}{2 N_{c}} \operatorname{Tr}\left(\left[F^{a_{1}}, F^{a_{2}}\right]\left[F^{a_{3}}, F^{a_{4}}\right]\right) \\
& \left(F^{a_{2}} F^{a_{3}} F^{a_{4}}\right)_{a_{1} a_{5}}=\frac{1}{2 N_{c}} \operatorname{Tr}\left(\left[\left[F^{a_{1}}, F^{a_{2}}\right], F^{a_{3}}\right]\left[F^{a_{4}}, F^{a_{5}}\right]\right) .
\end{aligned}
$$


It follows from Eq. (6.5) that, apart from the normalization factor, the identities obeyed by $F$ - and $T$-matrices are the same. Expanding out commutators in Eq. (6.5) and collecting identical terms using the cyclic property of the trace, we conclude that sets of traces of strings of $n$ color matrices in any representation with all $(n-1)$ ! non-cyclic permutations of the color labels of the gluons included, give a color basis that decomposes the tree level $n$-gluon amplitudes into colorless ordered amplitudes

$$
\begin{aligned}
& \mathcal{A}_{n}^{\text {tree }}=\frac{g_{s}^{n-2}}{2 N_{c}} \sum_{\sigma \in S_{n} / Z_{n}} \operatorname{Tr}\left(F^{a_{\sigma(1)}} F^{a_{\sigma(2)}} F^{a_{\sigma(3)}} \ldots F^{a_{\sigma(n)}}\right) A_{n, \sigma}^{\text {tree }}, \\
& \mathcal{A}_{n}^{\text {tree }}=g_{s}^{n-2} \sum_{\sigma \in S_{n} / Z_{n}} \operatorname{Tr}\left(T^{a_{\sigma(1)}} T^{a_{\sigma(2)}} T^{a_{\sigma(3)}} \ldots T^{a_{\sigma(n)}}\right) A_{n, \sigma}^{\text {tree }} .
\end{aligned}
$$

In the above equations, the sum runs over all non-cyclic permutations of $n$ gluons and $g_{s}$ is the QCD coupling constant. Because of Bose symmetry, all color-ordered sub-amplitudes $A_{n, \sigma}^{\text {tree }}$ are described by a single function, computed for different permutations of its arguments

$$
A_{n, \sigma}^{\text {tree }}=m_{n}\left(g_{\sigma(1)}, g_{\sigma(2)}, g_{\sigma(3)}, \ldots, g_{\sigma(n)}\right),
$$

where $g_{i}$ is a generic notation for an external gluon with momentum $k_{i}$ and helicity $h_{i}$. Since, as follows from Eq. (6.4), the color factor of any diagram can be rewritten through traces of $F$ - or $T$-matrices by means of the same mathematical operations, the two bases define the same colorless ordered amplitudes. The computation of the function $m_{n}\left(g_{1}, g_{2}, \ldots, g_{n}\right)$ is simplified if one notices [28, 81] that only diagrams where gluons appear in the same order as their color factors in the color trace, can contribute to a particular color-ordered tree amplitude. In fact, these sub-amplitudes can be calculated without any reference to color degrees of freedom, using color-stripped Feynman rules based on ordered colorless vertices $[84,83,85]$. We show the color-stripped Feynman rules in Fig. 6.1. As the name implies, these Feynman rules yield color-ordered Feynman diagrams where the color part has been stripped off. ${ }^{6}$ They can also be used to calculate colorless ordered amplitudes using, for instance, Berends-Giele recursion relations.

We turn to the discussion of properties of color-ordered tree amplitudes. Gauge invariance of the full amplitude $\mathcal{A}_{n}^{\text {tree }}$ and the fact that the color factors in Eqs. $(6.7,6.8)$ are linearly independent, ensure that the sub-amplitudes $m_{n}$ are gauge invariant for each gluon permutation separately. In addition, they obey a number of relations. The cyclic identity

$$
m_{n}\left(g_{1}, g_{2}, g_{3}, \ldots, g_{n}\right)=m_{n}\left(g_{2}, g_{3}, \ldots, g_{n}, g_{1}\right),
$$

${ }^{6}$ Note that the sign in the quark-gluon-antiquark vertex depends on the orientation of the diagram since $\left(T^{a}\right)_{i \bar{\jmath}}$ is the color matrix for a quark $-\left(T^{* a}\right)_{j \bar{\imath}}$ is the color matrix for an antiquark. 


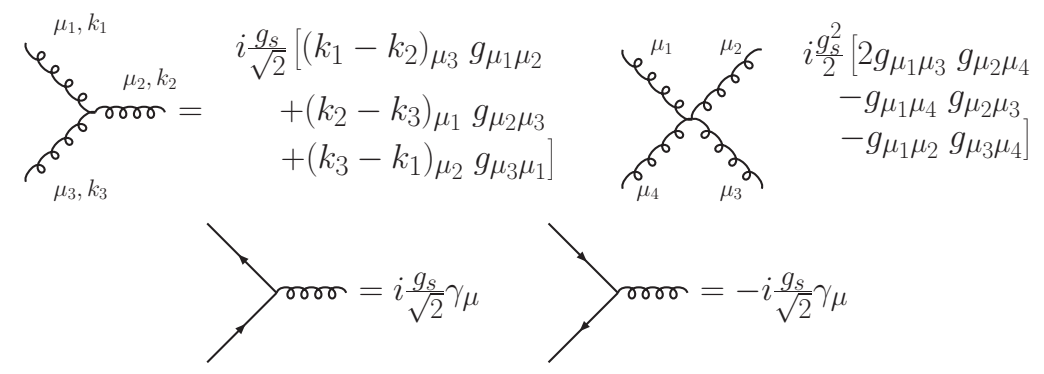

Figure 6.1: Color-ordered Feynman rules. All gluon momenta are outgoing.

follows from Eqs. $(6.2,6.1)$, if we use the linear independence and the cyclic properties of the color trace. The reflection identity

$$
m_{n}\left(g_{1}, g_{2}, g_{3}, \ldots, g_{n-1}, g_{n}\right)=(-1)^{n} m_{n}\left(g_{n}, g_{n-1}, \ldots, g_{2}, g_{1}\right),
$$

follows from the antisymmetry of the $S U(3)$ generators in the adjoint representation $F_{b c}^{a}=-F_{c b}^{a}$ and the related identity for color traces $\operatorname{Tr}\left(F^{a_{1}} F^{a_{2}} \ldots F^{a_{n}}\right)=$ $(-1)^{n} \operatorname{Tr}\left(F^{a_{n}} \ldots F^{a_{2}} F^{a_{1}}\right)$.

It is straightforward to verify that Eqs. $(6.10,6.11)$ reduce the number of independent color-ordered tree amplitudes in the pure gluon case from $n$ ! to $(n-1) ! / 2$. However, as we now show, more relations exist between the various color-ordered amplitudes. In particular, relations between different colorordered amplitudes arise if we consider color ordering in a theory with the gauge group given by the direct product of the two gauge $S U\left(N_{c}\right)$ groups [86]. The assumption that the two groups in the direct product are the same is not essential, but we will use it in what follows. We note that gauge fields in such a theory are charged under either one or the other gauge group, but not under both. The Lagrangian of the theory is the sum of two terms, each containing the square of the field strength tensor for the appropriate gluon field. Therefore the "gluons" of the two gauge groups do not interact with each other.

Given the Lagrangian of the theory, it is clear that scattering amplitudes that involve gluons of both types should vanish. This decoupling feature is perfectly obvious at the Lagrangian level, but it becomes obscure once the color ordering is performed. Indeed, the Lie algebra of the extended gauge group $S U\left(N_{c}\right) \otimes$ $S U\left(N_{c}\right)$ is defined by generators $\mathcal{T}^{a}, a=1,2, \ldots, 2\left(N_{c}^{2}-1\right)$. Those generators satisfy the commutation relations $\left[\mathcal{T}^{a}, \mathcal{T}^{b}\right]=i g^{a b c} \mathcal{T}^{c}$, where $g^{a b c}=\sqrt{2} f^{a b c}$ provided that $[a, b, c] \in S_{1}=\left[1, \ldots, N_{c}^{2}-1\right]$ or $[a, b, c] \in S_{2}=\left[N_{c}^{2}, \ldots, 2\left(N_{c}^{2}-1\right)\right]$ and $g^{a b c}=0$ otherwise. We now consider a scattering amplitude of gluons in the theory with such a gauge group; the interaction vertices are specified in terms of the structure constants $g^{a b c}$. The resulting color decomposition of amplitudes is identical to that in Eq. (6.8) up to an obvious replacement $T^{a} \rightarrow \mathcal{T}^{a}$. Of course, the color-stripped sub-amplitudes are, by their nature, unaffected by the changes in the color gauge group and, therefore, remain the same. We 
obtain

$$
\mathcal{A}_{n}^{\text {tree }}=g_{s}^{n-2} \sum_{\sigma \in S_{n} / Z_{n}} \operatorname{Tr}\left(\mathcal{T}^{a_{\sigma(1)}} \mathcal{T}^{a_{\sigma(2)}} \mathcal{T}^{a_{\sigma(3)}} \ldots \mathcal{T}^{a_{\sigma(n)}}\right) m_{n}\left(g_{\sigma(1)}, \ldots, g_{\sigma(n)}\right) .
$$

We now consider the scattering amplitude of $n_{1}$ gluons charged under the first gauge group and $n-n_{1}$ gluons charged under the second gauge group. As we already explained, the resulting scattering amplitude must vanish. This feature is not apparent from Eq. (6.12). In fact, as we show now, the vanishing of the full amplitude implies non-trivial identities between different colorless sub-amplitudes.

To see how these relations appear, we note that color factors on the right hand side of Eq. (6.12) can be simplified since Lie algebra generators with indices in the two lists $S_{1}$ and $S_{2}$ commute with each other

$$
\left[\mathcal{T}^{a}, \mathcal{T}^{b}\right]=0, \quad a \in S_{1}, \quad b \in S_{2} .
$$

Hence, the color weights in Eq. (6.12) can be re-written by commuting all generators $\mathcal{T}^{a_{1}}$ with $a_{1} \in S_{1}$ to the left of the color trace; of course, the relative ordering of gluons with indices in the same list can not be changed. Upon performing this procedure several times, we arrive at the color weights of the following form

$$
\operatorname{Tr}\left(\mathcal{T}^{a_{\sigma(1)}} \ldots \mathcal{T}^{a_{\sigma\left(n_{1}\right)}} \times \mathcal{T}^{b_{\sigma\left(n_{1}+1\right)}} \ldots \mathcal{T}^{b_{\sigma(n)}}\right),
$$

with $a_{i} \in S_{1}$ and $b_{i} \in S_{2}$. Since these color weights are independent, their coefficients must vanish. These coefficients are given by sums of color-ordered amplitudes where orderings of gluons with indices from the lists $S_{1}$ and $S_{2}$ are kept fixed, while all permutations between entries of different lists are allowed. For this reason we conclude that the color-ordered amplitudes satisfy a set of identities

$$
m_{n}\left(1, \underline{2, \ldots n_{1}}, \overline{n_{1}+1, \ldots, n}\right) \equiv \sum_{\sigma(n)} m_{n}\left(g_{1}, g_{\sigma(2)}, g_{\sigma(3)}, \ldots, g_{\sigma(n)}\right)=0 .
$$

The sum in this equation runs over all mergings of the two lists $\left\{2, . ., n_{1}\right\}$ and $\left\{n_{1}+1, . ., n\right\}$, that preserve relative ordering of elements in each list. The two lists to be merged are denoted by under- and over-lining. For example, in case of $n=6$, we can place three gluons into one list and two gluons into the other list. The full set of permutations that we have to consider reads

$$
\begin{aligned}
\theta(234 \mid 56)= & {[(23456),(23546),(25346),(52346),(23564),} \\
& (25364),(52364),(25634),(52634),(56234)] .
\end{aligned}
$$

An interesting special case occurs if we consider a list $\theta(2 \mid 34 \ldots n)$ which corresponds to the direct product of $U(1)$ and $S U\left(N_{c}\right)$ gauge groups. The gauge boson of the $U(1)$ group is referred to as a "photon". The "photon" decoupling identities read

$$
\begin{gathered}
m_{n}(1, \underline{2}, \overline{3, \ldots, n}) \equiv m_{n}\left(g_{1}, g_{2}, g_{3}, \ldots, g_{n}\right)+m_{n}\left(1, g_{3}, g_{2}, \ldots, g_{n}\right) \\
+\ldots m_{n}\left(g_{1}, g_{3}, \ldots, g_{n}, g_{2}\right)=0 .
\end{gathered}
$$


It is clear that Eq. (6.15) gives a set of relations for scattering amplitudes; such relations are usually referred to as Abelian relations [86]. They reduce the number of independent color-ordered sub-amplitudes in pure gluon case from $(n-1) ! / 2$ to $(n-2)$ !. Simultaneous application of the reflective and Abelian identities leads to many new identities, such as e.g. the Kleiss-Kuijf relations [87]. The Kleiss-Kuijf relations can also be used to reduce the number of independent color-ordered amplitudes to $(n-2)$ ! and, in this sense, they are equivalent to the Abelian identities.

The color decomposition for gluon scattering amplitudes that involves $(n-2)$ ! truly independent color structures was first derived in Ref. [88]. It is remarkably simple

$$
\begin{gathered}
\mathcal{A}_{n}^{\text {tree }}(1,2,3, \ldots, n)=g_{s}^{n-2} \sum_{\sigma=\mathcal{P}(2,3, \ldots, n-1)}\left(F^{a_{\sigma(2)}} \ldots F^{a_{\sigma(n-1)}}\right)_{a_{1} a_{n}} \\
\times m_{n}\left(g_{1}, g_{\sigma(2)}, g_{\sigma(3)}, \ldots, g_{\sigma(n-1)}, g_{n}\right) .
\end{gathered}
$$

We sketch here a derivation of Eq. (6.18) which is based on the color commutator algebra, a simple decomposition of the sum over permutations and the Abelian identities [89]. We first introduce compact notations for products of color matrices

$$
\begin{aligned}
& \left\{a_{i_{1}} a_{i_{2}} \ldots a_{i_{n}}\right\}_{u v} \equiv\left(F^{a_{i_{1}}} F^{a_{i_{2}}} \ldots F^{a_{i_{n}}}\right)_{u v}, \\
& \left\{a_{i_{1}} a_{i_{2}} \ldots a_{i_{n}}\right\} \equiv \operatorname{Tr}\left(F^{a_{i_{1}}} F^{a_{i_{2}}} \ldots F^{a_{i_{n}}}\right) \\
& \{[a, b]\}_{c d} \equiv\left(\left[F^{a}, F^{b}\right]\right)_{c d}, \quad\{\}_{a b} \equiv \delta_{a b} .
\end{aligned}
$$

In the new notation Eq. (6.2) becomes

$$
\{[a, b]\}_{c d}=-\{a\}_{b y}\{y\}_{c d}, \quad\{a b\}=2 N_{c} \delta_{a b} .
$$

We start with a list of identities between the products of color matrices. With the use of the commutator Eq. (6.20) it is easy to derive

$$
\begin{aligned}
\left\{a_{2} a_{1}\right\}_{u v}=\{ & \left.a_{1} a_{2}\right\}_{u v}-\left\{a_{2}\right\}_{a_{1} y}\{y\}_{u v} \\
\left\{a_{2} a_{3} a_{1}\right\}_{u v}= & \left\{a_{1} a_{2} a_{3}\right\}_{u v}-\left\{a_{2}\right\}_{a_{1} y}\left\{y a_{3}\right\}_{u v}-\left\{a_{3}\right\}_{a_{1} y}\left\{y a_{2}\right\}_{u v} \\
& +\left\{a_{3} a_{2}\right\}_{a_{1} y}\{y\}_{u v} \\
\left\{a_{2} a_{3} a_{4} a_{1}\right\}_{u v} & =\left\{a_{1} a_{2} a_{3} a_{4}\right\}_{u v}-\left\{a_{2}\right\}_{a_{1} y}\left\{y a_{3} a_{4}\right\}_{u v} \\
& -\left\{a_{3}\right\}_{a_{1} y}\left\{y a_{2} a_{4}\right\}_{u v}-\left\{a_{4}\right\}_{a_{1} y}\left\{y a_{2} a_{3}\right\}_{u v} \\
& +\left\{a_{3} a_{2}\right\}_{a_{1} y}\left\{y a_{4}\right\}_{u v}+\left\{a_{4} a_{2}\right\}_{a_{1} y}\left\{y a_{3}\right\}_{u v} \\
& +\left\{a_{4} a_{3}\right\}_{a_{1} y}\left\{y a_{3}\right\}_{u v}-\left\{a_{4} a_{3} a_{2}\right\}_{y a_{1}}\{y\}_{u v} .
\end{aligned}
$$

The generalization of these identities to products of an arbitrary number of color matrices can be easily deduced from Eq. (6.21).

As a next step, we consider the four-gluon scattering amplitude and show how to verify Eq. (6.18) in that case. This is a good example since the algebra in the four-gluon case is easy but the calculation generalizes to the $n$-gluon 
case. We express the four-gluon scattering amplitude through color-ordered amplitudes fixing the position of the fourth gluon and keeping the sum over the permutations of the second and third gluons. We use a simplified notation for the sum over permutations and write

$$
\begin{aligned}
\mathcal{A}_{4}^{\text {tree }}= & \frac{g_{s}^{2}}{2 N_{c}} \sum_{\mathcal{P}(2,3)}\left[\left\{a_{1} a_{2} a_{3} a_{4}\right\} m_{4}\left(g_{1}, g_{2}, g_{3}, g_{4}\right)\right. \\
& \left.+\left\{a_{2} a_{1} a_{3} a_{4}\right\} m_{4}\left(g_{2}, g_{1}, g_{3}, g_{4}\right)+\left\{a_{2} a_{3} a_{1} a_{4}\right\} m_{4}\left(g_{2}, g_{3}, g_{1}, g_{4}\right)\right] .
\end{aligned}
$$

We employ relations shown in Eq. (6.21) to move the color matrix $a_{1}$ to the left; this generates seven terms on the right hand side in Eq. (6.22). Collecting common color factors, we obtain

$$
\begin{aligned}
& \mathcal{A}_{4}^{\text {tree }}=\frac{g_{s}^{2}}{2 N_{c}} \sum_{\mathcal{P}(2,3)}\left[\left\{a_{1} a_{2} a_{3} a_{4}\right\} m_{4}(\underline{1}, \overline{2,3}, 4)\right. \\
& \left.+\left\{a_{2} a_{3}\right\}_{a_{1} y}\left\{y a_{4}\right\} m_{4}\left(g_{2}, g_{3}, g_{1}, g_{4}\right)-\left\{a_{2}\right\}_{a_{1} y}\left\{y a_{3}, a_{4}\right\} m_{4}(\underline{2,1}, \overline{3}, 4)\right] .
\end{aligned}
$$

The first and the last terms on the right hand side of Eq. (6.23) vanish because of the Abelian identities, $m_{4}(\underline{1}, \overline{2,3}, 4)=0$ and $m_{4}(2,1, \overline{3}, 4)=0$. Finally, we use $\left\{y a_{4}\right\}=2 N_{c} \delta_{y a_{4}}$ and the cyclic and reflection identities to transform Eq. (6.23) to the desired result

$$
\mathcal{A}_{4}^{\text {tree }}=g_{s}^{2} \sum_{\mathcal{P}(2,3)}\left\{a_{2} a_{3}\right\}_{a_{1} a_{4}} m_{4}\left(g_{1}, g_{2}, g_{3}, g_{4}\right) .
$$

It is easy to generalize this proof to the $n$-gluon case. We write

$$
\begin{aligned}
\mathcal{A}_{n}^{\text {tree }} & =\frac{g_{s}^{n-2}}{2 N_{c}} \sum_{\mathcal{P}(1, \cdots, n-1)}\left\{a_{1} a_{2} \cdots a_{n}\right\} m_{n}\left(g_{1}, g_{2}, \ldots, g_{n}\right) \\
& =\frac{g_{s}^{n-2}}{2 N_{c}} \sum_{\mathcal{P}(2, \cdots, n-1)}\left[\left\{a_{1} a_{2} \cdots a_{n}\right\} m_{n}\left(g_{1}, g_{2}, \ldots, g_{n}\right)\right. \\
& \left.+\sum_{k=2}^{n-1}\left\{a_{2} \cdots a_{k} a_{1} a_{k+1} \cdots, n\right\} m_{n}\left(g_{2}, \ldots, g_{k}, g_{1}, g_{k+1}, \ldots, g_{n}\right)\right],
\end{aligned}
$$

and, collecting the relevant terms, obtain

$$
\begin{aligned}
\mathcal{A}_{n}^{\text {tree }} & =\frac{g_{s}^{n-2}}{2 N_{c}} \sum_{\mathcal{P}(2, \cdots, n-1)}\left[\left\{a_{1} a_{2} \cdots a_{n}\right\} m_{n}(\underline{1}, \overline{2, \ldots, n-1}, n)\right. \\
& +\sum_{k=2}^{n-2}(-1)^{k+1}\left\{a_{2} \cdots a_{k}\right\}_{a_{1} y}\left\{y a_{k+1} \cdots a_{n}\right\} \\
& \left.\times m_{n} \frac{(k, \ldots, 2,1}{k+1, \ldots, n-1}, n\right) \\
& \left.+(-1)^{n}\left\{a_{2} \cdots a_{n-1}\right\}_{a_{1} y}\left\{y a_{n}\right\} m_{n}\left(g_{n-1}, \ldots, g_{1}, g_{n}\right)\right] .
\end{aligned}
$$


Similar to the four-gluon case, because of the Abelian identities, (Eq. (6.15)), only the last term on the right hand side in Eq. (6.26) does not vanish. Application of the reflection and cyclic identities gives

$$
\begin{aligned}
\mathcal{A}_{n}^{\text {tree }} & =\frac{g_{s}^{n-2}}{2 N_{c}} \sum_{\mathcal{P}(2, \cdots, n-1)}\left\{a_{2} \cdots a_{n-1}\right\}_{a_{1} y}\left\{y a_{n}\right\} m_{n}\left(g_{1}, g_{2}, \ldots, g_{n-1}, g_{n}\right) \\
& =g_{s}^{n-2} \sum_{\mathcal{P}(2, \cdots, n-1)}\left\{a_{2} \cdots a_{n-1}\right\}_{a_{1} a_{n}} m_{n}\left(g_{1}, g_{2}, \ldots, g_{n-1}, g_{n}\right) .
\end{aligned}
$$

It follows from the derivation that we just presented that the colorless ordered amplitudes in this representation are the same as in the color decompositions using $F$ and $T$ bases.

An important feature of this color basis is that two of the color indices do not appear in the permutation sum. Hence, this color basis is not manifestly symmetric. However, it is a unitary basis in the sense that on each pole of the tree amplitude the color factor of a given colorless ordered amplitude also factorizes. Indeed, poles in tree color-ordered amplitudes appear when a linear combination of momenta of some set of neighboring gluons becomes light-like. For definiteness, we assume that those gluons are $g_{1}, . ., g_{m}$. The color-ordered amplitude then factorizes into the product of two amplitudes

$$
m_{n}\left(g_{1}, . ., g_{n}\right) \rightarrow k_{v}^{-2} m_{m+1}\left(g_{1}, \ldots, g_{m}, g_{v}\right) m_{n-m+1}\left(g_{v}, g_{m+1}, \ldots, g_{n}\right),
$$

where $k_{v}=-\sum_{i=1}^{m} k_{i}$ and the summation over the helicities of the intermediate gluon is assumed. The "unitary" nature of the color decomposition in Eq. (6.27) can be best seen if we associate natural color factors with the three amplitudes in Eq. (6.28) and sum over colors of the internal gluon. The color factor that we associate with the right-hand side of Eq. (6.28) is

$$
\sum_{a_{v}}\left\{a_{2} \ldots a_{m}\right\}_{a_{1} a_{v}}\left\{a_{m+1}, \ldots . a_{n-1}\right\}_{a_{v} a_{n}}=\left\{a_{2} \ldots a_{n-1}\right\}_{a_{1} a_{n}}
$$

which is indeed the color factor of the full amplitude $m_{n}\left(g_{1}, . ., g_{n}\right)$.

The above argument explains why the color basis in Eq. (6.27) is particularly suitable for one-loop computations, using generalized unitarity. Indeed, in the context of generalized unitarity, we expect to reconstruct one-loop amplitudes from tree amplitudes. Summation over color indices of intermediate cut lines is performed in exactly the same manner as in the tree level case, described above. As a result, we expect that the color basis in Eq. (6.27) remains valid also when one-loop amplitudes are considered. To see this explicitly, we write the full amplitude as a sum of terms where gluons are ordered

$$
\mathcal{A}_{n}^{1-\text { loop }}=g_{s}^{n} c_{\Gamma} \sum_{\sigma \in S_{n-1}} A_{n}^{(1)}\left(g_{1}, g_{\sigma(2)}, \ldots, g_{\sigma(n)}\right),
$$


The factor $c_{\Gamma}$ is the standard term that appears in dimensionally regulated one-loop calculations,

$$
c_{\Gamma}=\frac{1}{(4 \pi)^{2-\epsilon}} \frac{\Gamma(1+\epsilon) \Gamma^{2}(1-\epsilon)}{\Gamma(1-2 \epsilon)}=\frac{(4 \pi)^{\epsilon}}{16 \pi^{2}} \frac{1}{\Gamma(1-\epsilon)} .
$$

We now consider a double-cut of one of the terms in the sum in Eq. (6.30). The cut splits the ordered momenta in two groups, say $[1, . ., k]$ and $[k+1, . ., n]$. We write the imaginary part of the amplitude in that channel as

$$
\begin{aligned}
\operatorname{Im}_{(k, n)} & {\left[A_{n}^{(1)}\left(g_{1}, g_{2}, \ldots, g_{n}\right)\right]=\left\{a_{1} a_{2} \cdots a_{k}\right\}_{v u}\left\{a_{k+1} \cdots a_{n}\right\}_{u v} } \\
& \times m_{k+2}\left(g_{v}, g_{1}, g_{2}, \ldots, g_{k}, g_{u}\right) m_{n-k+2}\left(g_{u}, g_{k+1}, \ldots, g_{n}, g_{v}\right) \\
& =\left\{a_{1} a_{2} \cdots a_{n}\right\} \operatorname{Im}_{(k, n)}\left[m_{n}^{(1)}\left(g_{1}, g_{2}, \ldots, g_{n}\right)\right] .
\end{aligned}
$$

The color decomposition of the one-loop amplitude is independent of whether we take the imaginary part or not. We conclude that one-loop gluon amplitudes ${ }^{7}$ obey a color decomposition of the following form [88]

$$
\mathcal{A}_{n}^{1-\text { loop }}=g_{s}^{n} c_{\Gamma} \sum_{\mathcal{P}(2, \cdots, n) / \mathcal{R}}\left\{F^{a_{1}}, \ldots, F^{a_{n}}\right\} m_{n}^{(1)}\left(g_{1}, g_{2}, \ldots, g_{n}\right) .
$$

where $\mathcal{R}$ is the reflection transformation, which needs to be factored out to remove a symmetry factor that appears otherwise. The cyclic property and reflection symmetry remain valid. Hence, we conclude that the number of independent one-loop amplitudes is $(n-1) ! / 2$.

Before finishing this subsection we note that the color-ordered tree amplitudes also satisfy the Bern-Carrasco-Johansson (BCJ) relation [90]. The BCJ relations for gluon amplitudes read

$$
\begin{gathered}
s_{12} m_{n}\left(g_{1}, g_{2}, g_{3}, \ldots, g_{n}\right)+\left(s_{12}+s_{23}\right) m_{n}\left(g_{1}, g_{3}, g_{2}, g_{4}, \ldots, g_{n}\right)+\cdots \\
+\left(s_{12}+s_{23}+\cdots s_{2(n-1)}\right) m_{n}\left(g_{1}, g_{3}, g_{4}, \ldots, g_{n-1}, g_{2}, g_{n}\right)=0
\end{gathered}
$$

where $s_{i j}=2 k_{i} \cdot k_{j}$. They have been proven first using the field theory limit of monodromy relations in string theory [91] and later derived using BCFW recursion relations $[92,93]^{8}$. When all relations between different color-ordered gluon scattering amplitudes are combined, the number of independent colorordered amplitudes reduces to $(n-3)$ !.

\section{2. $\bar{q} q+(n-2)$-gluon amplitudes}

The color parts of Feynman diagrams that contribute to tree $q \bar{q}+(n-2)$ gluon amplitudes have the generic form of a product of color matrices in the

\footnotetext{
${ }^{7}$ In this subsection we only deal with $S U\left(N_{c}\right)$ pure gauge theory and do not consider matter fields.

${ }^{8}$ In Ref. [92] the reflection identity, the Abelian identities and the BCJ relations are derived using only $\mathrm{BCFW}$ recursion relations.
} 
fundamental representation contracted in their adjoint indices with products of color matrices in the adjoint representation

$$
\left(T^{b_{1}} \ldots T^{b_{k}} \ldots\right)_{j \bar{\imath}} \times\left(F^{a_{1}} \ldots F^{a_{r}}\right)_{b_{1} a_{r+1}} \ldots\left(F^{a_{p}} \ldots F^{a_{t}-1}\right)_{b_{k} a_{t}} \ldots
$$

The product of $F$-matrices can be converted into traces of multiple commutators of the $S U\left(N_{c}\right)$ generators in the fundamental representation using Eq. (6.5). In order to convert complicated expressions to simple products of $T$-matrices, we can use the identity

$$
\left(T_{X} T^{a} T_{Y}\right)_{j \bar{\imath}} \operatorname{Tr}\left(\left[T^{a}, T^{b}\right] T_{Z}\right)=\left(T_{X}\left[T^{b}, T_{Z}\right] T_{Y}\right)_{j \bar{\imath}},
$$

where $T_{X, Y, Z}$ denote generic products of $T$ matrices. Therefore, we conclude that all color factors of the $\bar{q} q+n$ gluon amplitudes can be transformed into a linear combination of products of $T$-matrices $\left(T^{a_{i_{1}}} \ldots T^{a_{i_{n}}}\right)_{j \bar{\imath}}$. All the independent terms of such type form a color basis for the decomposition of tree amplitudes

$$
\begin{aligned}
\mathcal{A}_{n}^{\text {tree }}\left(\bar{q}_{1}, q_{2}, g_{3}, \ldots, g_{n}\right)= & g_{s}^{n-2} \sum_{\sigma \in S_{n-2}}\left(T^{a_{\sigma(3)}} T^{a_{\sigma(4)}} \ldots T^{a_{\sigma(n)}}\right)_{i_{2} \bar{\imath}_{1}} \\
& \times m_{n}\left(\bar{q}_{1}, q_{2}, g_{\sigma(3)}, \ldots, g_{\sigma(n)}\right) .
\end{aligned}
$$

In Eq. $(6.37), m_{n}\left(\bar{q}_{1}, q_{2}, g_{\sigma(3)}, \ldots, g_{\sigma(n)}\right)$ stands for the colorless ordered tree amplitude for $\bar{q} q+(n-2)$-gluon scattering. In conjunction with two-particle unitarity cuts, Eq. (6.37) can be used to obtain the color decomposition of a quark-loop contribution to one-loop gluon-gluon scattering amplitude

$$
\mathcal{A}_{n ; n_{f}}^{1-\text { loop }}=g_{s}^{n} c_{\Gamma} n_{f} \sum_{\sigma \in S_{n-1}} \operatorname{Tr}\left(T^{a_{1}} T^{a_{\sigma(2)}} \ldots T^{a_{\sigma n}}\right) m_{n ; n_{f}}^{(1)}\left(g_{1}, g_{\sigma(2)}, \ldots, g_{\sigma(n)}\right),
$$

where $n_{f}$ is the number of quark flavors. In QCD the full one-loop $n$-gluon amplitude is the sum of the right hand sides of Eq. (6.33) and Eq. (6.38).

The color basis of Eq. (6.37) for the $\bar{q}+q+(n-2)$-gluon amplitudes keeps the quark and anti-quark indices fixed in the sense that they do not participate in the permutation sum. Using the commutator identities we will transform this color basis to another one, where the positions of one gluon and the anti-quark are kept fixed, while the positions of the quark and other gluons are arbitrary. As we will show, this new basis is an unitary color basis and, because of that, it is well-suited for constructing the color decomposition of one-loop amplitudes.

We begin by extending the compact notations introduced in Eq. (6.19) for the products of $F$-matrices, to also describe products of $T$-matrices

$$
\begin{aligned}
& \left(a_{i_{1}} a_{i_{2}} \ldots a_{i_{n}}\right)_{j \bar{\imath}} \equiv\left(T^{a_{i_{1}}} T^{a_{i_{2}}} \ldots T^{a_{i_{n}}}\right)_{j \bar{\imath}}, \\
& \left(a_{i_{1}} a_{i_{2}} \ldots a_{i_{n}}\right) \equiv \operatorname{Tr}\left(T^{a_{i_{1}}} T^{a_{i_{2}}} \ldots T^{a_{i_{n}}}\right), \\
& ([a, b])_{j \bar{\imath}}=-\{a\}_{b y}(y)_{j \bar{\imath}}, \quad(a b) \equiv \delta_{a b} .
\end{aligned}
$$


The commutator identities will have the same structure as in the pure gluon case (6.21) except that curly brackets will have to be replaced by ordinary ones, when products of $T$-matrices are involved. We find

$$
\begin{aligned}
&\left(a_{2} a_{1}\right)_{j \bar{\imath}}=\left(a_{1} a_{2}\right)_{j \bar{\imath}}-\left\{a_{2}\right\}_{a_{1} y}(y)_{j \bar{\imath}}, \\
&\left(a_{2} a_{3} a_{1}\right)_{j \bar{\imath}}=\left(a_{1} a_{2} a_{3}\right)_{j \bar{\imath}}-\left\{a_{2}\right\}_{a_{1} y}\left(y a_{3}\right)_{j \bar{\imath}} \\
&-\left\{a_{3}\right\}_{a_{1} y}\left(y a_{2}\right)_{j \bar{\imath}}+\left\{a_{3} a_{2}\right\}_{a_{1} y}(y)_{j \bar{\imath}}, \\
&\left(a_{2} a_{3} a_{4} a_{1}\right)_{j \bar{\imath}}=\left(a_{1} a_{2} a_{3} a_{4}\right)_{j \bar{\imath}}-\left\{a_{2}\right\}_{a_{1} y}\left(y a_{3} a_{4}\right)_{j \bar{\imath}} \\
&-\left\{a_{3}\right\}_{a_{1} y}\left(y a_{2} a_{4}\right)_{j \bar{\imath}}-\left\{a_{4}\right\}_{a_{1} y}\left(y a_{2} a_{3}\right)_{j \bar{\imath}} \\
&+\left\{a_{3} a_{2}\right\}_{a_{1} y}\left(y a_{4}\right)_{j \bar{\imath}}+\left\{a_{4} a_{2}\right\}_{a_{1} y}\left(y a_{3}\right)_{j \bar{\imath}} \\
&+\left\{a_{4} a_{3}\right\}_{a_{1} y}\left(y a_{2}\right)_{j \bar{\imath}}-\left\{a_{4} a_{3} a_{2}\right\}_{y a_{1}}(y)_{j \bar{\imath}} .
\end{aligned}
$$

As a first simple step, we consider the case of the $\bar{q} q+3$ gluon amplitude. We write permutations of gluon $g_{3}$ explicitly

$$
\begin{gathered}
\mathcal{A}_{5}^{\text {tree }}\left(\bar{q}_{1}, q_{2}, g_{3}, g_{4}, g_{5}\right)=g_{s}^{3} \sum_{\mathcal{P}(3,4,5)}\left(a_{3} a_{4} a_{5}\right)_{i_{2} \bar{\imath}_{1}} m_{5}\left(\bar{q}_{1}, q_{2}, g_{3}, g_{4}, g_{5}\right) \\
=g_{s}^{3} \sum_{\mathcal{P}(4,5)}\left[\left(a_{3} a_{4} a_{5}\right)_{i_{2} \bar{\imath}_{1}} m_{5}\left(\bar{q}_{1}, q_{2}, g_{3}, g_{4}, g_{5}\right)\right. \\
\left.+\left(a_{4} a_{3} a_{5}\right)_{i_{2} \bar{\imath}_{1}} m_{5}\left(\bar{q}_{1}, q_{2}, g_{4}, g_{3}, g_{5}\right)+\left(a_{4} a_{5} a_{3}\right)_{i_{2} \bar{\imath}_{1}} m_{5}\left(\bar{q}_{1}, q_{2}, g_{4}, g_{5}, g_{3}\right)\right],
\end{gathered}
$$

and use the color identities Eq. (6.40) to move the color index $a_{3}$ to the first position. Upon doing that, we find that the first term in Eq. (6.41) does not change, the second term generates two terms and the third generates four terms. We can combine those terms by exploiting the fact that there is a summation over the permutations of gluons $g_{4}$ and $g_{5}$ in Eq. (6.41), which allows us to interchange them. We obtain

$$
\begin{aligned}
& \mathcal{A}_{5}^{\text {tree }}\left(\bar{q}_{1}, q_{2}, g_{3}, g_{4}, g_{5}\right)=g_{s}^{3} \sum_{\mathcal{P}(4,5)}\left[\{\}_{a_{3} y}\left(y a_{4} a_{5}\right)_{i_{2} \bar{\imath}_{1}} m_{5}\left(\bar{q}_{1}, q_{2}, \underline{3}, \overline{4,5}\right)\right. \\
& \left.-\left\{a_{4}\right\}_{a_{3} y}\left(y a_{5}\right)_{i_{2} \bar{\imath}_{1}} m_{5}\left(\bar{q}_{1}, q_{2}, \underline{4,3}, \overline{5}\right)+\left\{a_{4} a_{5}\right\}_{a_{3} y}(y)_{i_{2} \bar{\imath}_{1}} m_{5}\left(\bar{q}_{1}, q_{2}, g_{5}, g_{4}, g_{3}\right)\right] .
\end{aligned}
$$

To rewrite Eq. (6.42) in a way that allows further generalization, it is convenient to introduce the notation

$$
\begin{aligned}
& \tilde{m}_{5}\left(\bar{q}_{1}, g_{5}, g_{4}, q_{2}, g_{3}\right)=-m_{5}\left(\bar{q}_{1}, q_{2}, \underline{3}, \overline{4,5}\right), \\
& \tilde{m}_{5}\left(\bar{q}_{1}, g_{5}, q_{2}, g_{4}, g_{3}\right)=m_{5}\left(\bar{q}_{1}, q_{2}, \underline{4,3}, \overline{5}\right), \\
& \tilde{m}_{5}\left(\bar{q}_{1}, q_{2}, g_{5}, g_{4}, g_{3}\right)=-m_{5}\left(\bar{q}_{1}, q_{2}, g_{5}, g_{4}, g_{3}\right) .
\end{aligned}
$$

We note that the two lists of gluons, whose members can be permuted, are separated in the argument of amplitudes $\tilde{m}_{5}$ by $\bar{q}$ and $q$ labels. A convenient way to visualize these amplitudes is to imagine that the quark line runs horizontally, from $\bar{q}$ to $q$ and that, in the argument of $\tilde{m}_{5}$, gluons that are to the right of $\bar{q}$ 
and to the left of $q$ are drawn below the quark line, while gluons to the right of $q$ are drawn above that line. We will use this way of visualizing these objects in Sect. 7.3 when we discuss the Berends-Giele recursion relations for colorless tree amplitudes.

We write our final result for the full five-point amplitude as

$$
\begin{aligned}
& \mathcal{A}_{5}^{\text {tree }}\left(\bar{q}_{1}, q_{2}, g_{3}, g_{4}, g_{5}\right)=-g_{s}^{3} \sum_{\mathcal{P}(4,5)}\left[\{\}_{a_{3} y}\left(y a_{4} a_{5}\right)_{i_{2} \bar{\imath}_{1}} \tilde{m}_{5}\left(\bar{q}_{1}, g_{5}, g_{4}, q_{2}, g_{3}\right)(6.44)\right. \\
& \left.+\left\{a_{4}\right\}_{a_{3} y}\left(y a_{5}\right)_{i_{2} \bar{\imath}_{1}} \tilde{m}_{5}\left(\bar{q}_{1}, g_{5}, q_{2}, g_{4}, g_{3}\right)+\left\{a_{4} a_{5}\right\}_{a_{3} y}(y)_{i_{2} \bar{\imath}_{1}} \tilde{m}_{5}\left(\bar{q}_{1}, q_{2}, g_{5}, g_{4}, g_{3}\right)\right] .
\end{aligned}
$$

Note that the positions of the first gluon and the anti-quark are fixed, while we have a permutation sum over the positions of the other gluons and the quark. This pattern remains valid also for $n$-gluons. We introduce a special notation for objects that multiply color structures in $\bar{q} q+n$ gluon scattering amplitude

$$
\tilde{m}_{n}\left(\bar{q}_{1}, g_{n}, . ., g_{(k+1)}, q_{2}, g_{k}, . ., g_{3}\right)=(-1)^{k} m_{n}\left(\bar{q}_{1}, q_{2}, \underline{k, \ldots, 3}, \overline{(k+1), . ., n}\right) .
$$

The color decomposition in the new basis reads

$$
\begin{aligned}
& \mathcal{A}_{n}^{\text {tree }}\left(\bar{q}_{1}, q_{2}, g_{3}, \ldots, g_{n}\right)=g_{s}^{n-2}(-1)^{n} \sum_{k=3}^{n} \sum_{\mathcal{P}(4, \ldots, n)}\left(y a_{\sigma(k+1)} . . a_{\sigma(n)}\right)_{i_{2} \bar{\imath}_{1}} \\
& \times\left\{a_{\sigma(4)} \ldots a_{\sigma(k)}\right\}_{a_{3} y} \tilde{m}_{n}\left(\bar{q}_{1}, g_{\sigma(n)}, \ldots, g_{\sigma(k+1)}, q_{2}, g_{\sigma(k)}, \ldots, g_{3}\right) .
\end{aligned}
$$

It is clear from the derivation that alternative color decompositions are possible, e.g. by fixing positions of the quark $q$ and the gluon $g_{n}$, instead of $g_{1}$ and $\bar{q}$. In this case we obtain

$$
\begin{aligned}
& \mathcal{A}_{n}^{\text {tree }}\left(\bar{q}_{1}, q_{2}, g_{3}, . ., g_{n}\right)=(-1)^{n} g_{s}^{n-2} \sum_{k=2}^{n-1} \sum_{\mathcal{P}(3, . ., n-1)}\left(a_{\sigma(3)} . . a_{\sigma(k)} y\right)_{i_{2} \bar{\imath}_{1}}(6.47) \\
& \times\left\{a_{\sigma(k+1)} . . a_{\sigma(n-1)}\right\}_{y a_{n}} \tilde{m}_{n}\left(\bar{q}_{1}, g_{\sigma(k)}, . ., g_{\sigma(3)}, q_{2}, g_{n}, g_{\sigma(n-1)}, . ., g_{\sigma(k+1)}\right) .
\end{aligned}
$$

We shall refer to the new fully ordered amplitudes $\tilde{m}$ defined in Eq. (6.45), appearing in Eqs. $(6.46,6.47)$, as tree left and right primitive amplitudes. They are given by linear combinations of tree color-ordered amplitudes, so that the usefulness of these objects may not be immediately clear. Nevertheless, the primitive amplitudes are useful since they become basic objects in one-loop computations, as we explain below. Also, the primitive amplitudes can be computed in a straightforward way using Berends-Giele recurrence relations and color-stripped Feynman rules, see Sect. 7.3.

We can now explain why the color decomposition in terms of tree primitive amplitudes appearing in Eqs. $(6.46,6.47))$ is unitary. We begin by writing the full amplitude as a linear combination of sub-amplitudes where all particles are ordered

$$
\begin{aligned}
& \mathcal{A}_{n}^{1-\text { loop }}\left(\bar{q}_{1}, q_{2}, g_{3}, \ldots, g_{n}\right)= \\
& g_{s}^{n} c_{\Gamma} \sum_{\sigma \in S_{n-2}} \sum_{k=3}^{n} A^{(1)}\left(\bar{q}_{1}, g_{\sigma(3)}, \ldots, g_{\sigma(k)}, q_{2}, g_{\sigma(k+1)}, \ldots, g_{\sigma(n)}\right) .
\end{aligned}
$$




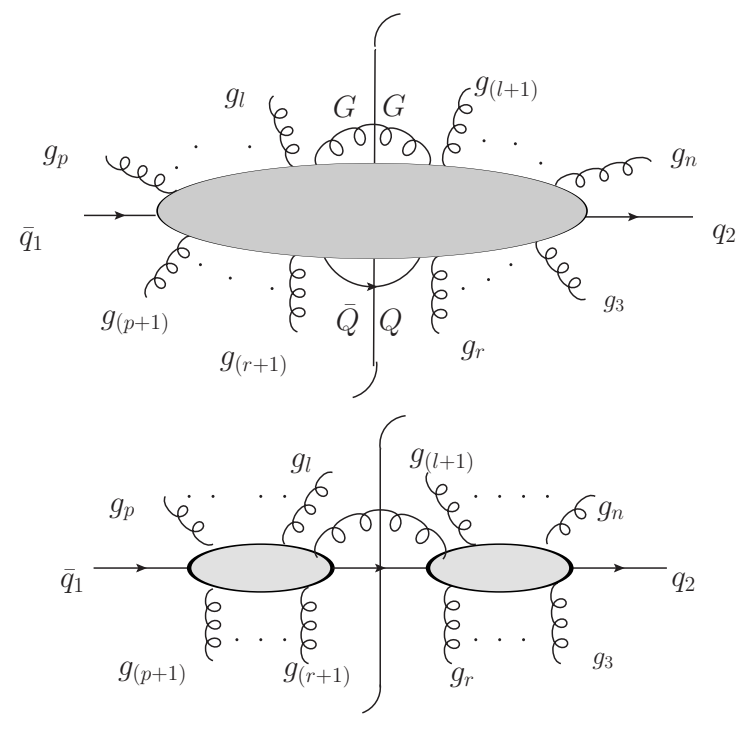

Figure 6.2: Quark-gluon cut of the ordered colorless one-loop amplitude Eq. (6.49).

Next, we consider the imaginary part of one of the ordered amplitudes $A^{(1)}\left(\bar{q}_{1}, g_{p},\right.$. . $\left.., g_{3}, q_{2}, g_{n}, \ldots, g_{p+1}\right)$ obtained by cutting a quark propagator between outgoing gluons at positions $r$ and $r+1$ with $r \leq p$ and a gluon propagator between gluons at positions $l$ and $l+1$ with $l \geq p+1$. The cut one-loop ordered amplitude is given by products of tree amplitudes and we choose the color representation where the positions of (anti-) quarks and gluons are not subject to permutations. Using the color bases of Eqs. (6.47) and (6.46), and suppressing summation over spin quantum numbers of the cut lines, we obtain the imaginary parts

$$
\begin{aligned}
\operatorname{Im}_{(r, l)} & {\left[A^{(1)}\left(\bar{q}_{1}, g_{p}, . ., g_{r+1}\left|{ }_{Q}\right|_{\bar{Q}} g_{r}, . ., g_{3}, q_{2}, g_{n}, . .,\left.g_{l+1}{ }_{G}\right|_{G} g_{l}, . ., g_{p+1}\right)\right] } \\
= & \left\{a_{p} . . a_{l}\right\}_{x_{2} a_{G}}\left(a_{r+1} . . a_{p+1} x_{2}\right)_{j} \bar{i}_{1}\left(x_{1} a_{3} . . a_{r}\right)_{i_{2}} \bar{j}\left\{a_{l+1} . . a_{n}\right\}_{a_{G} x_{1}} \\
& \times(-1)^{r+n-l} \tilde{m}\left(\bar{Q}, g_{r}, \ldots, g_{3}, q_{2}, g_{n}, \ldots, g_{l+1}, G\right) \\
& \times(-1)^{l-r} \tilde{m}\left(G, g_{l}, \ldots, g_{p}, \bar{q}_{1}, g_{p+1}, \ldots, g_{r+1}, Q\right) \\
= & (-1)^{n}\left(x_{1} a_{3} . . a_{p+1} x_{2}\right)_{i_{2} \bar{\imath}_{1}}\left\{a_{p} . . a_{n}\right\}_{x_{2} x_{1}} \\
& \times \operatorname{Im}_{(r, l)}\left[m_{n}^{(1)}\left(\bar{q}_{1}, g_{p}, . ., g_{3}, q_{2}, g_{n}, . ., g_{p+1}\right)\right] .
\end{aligned}
$$

The cut is illustrated in Fig. 6.2. Since the color decomposition of an amplitude does not depend on whether it is cut or not, from Eq. (6.49) we read off the 
following color decomposition [88] of a one-loop $q \bar{q}+n$ gluon amplitude

$$
\begin{aligned}
& \mathcal{A}_{n}^{1-\operatorname{loop}}\left(\bar{q}_{1}, q_{2}, g_{3}, . ., g_{n}\right)=g_{s}^{n} c_{\Gamma} \sum_{p=2}^{n} \sum_{\sigma \in S_{n-2}}\left(T^{x_{2}} T^{a_{\sigma_{3}}} . . T^{a_{\sigma_{p}}} T^{x_{1}}\right)_{i_{2} \bar{\tau}_{1}} \\
& \times\left(F^{a_{\sigma_{p+1}}} . . F^{a_{\sigma_{n}}}\right)_{x_{1} x_{2}}(-1)^{n} \tilde{m}_{n}^{(1)}\left(\bar{q}_{1}, g_{\sigma(p)}, . ., g_{\sigma(3)}, q_{2}, g_{\sigma(n)}, . ., g_{\sigma(p+1)}\right) .
\end{aligned}
$$

We note that for $p=2$ the factor $(T \cdots T)_{i_{2} \bar{\imath}_{1}}$ becomes $\left(T^{x_{2}} T^{x_{1}}\right)_{i_{2} \bar{\imath}_{1}}$ and for $p=n$ the factor $(F \cdots F)_{x_{1} x_{2}}$ becomes $\delta_{x_{1} x_{2}}$. We also note that $\tilde{m}_{n}^{(1)}$ is the left primitive amplitude introduced in Ref. [84]. Although we showed that this color decomposition of a one-loop amplitude for $\bar{q} q+n$ gluons is unitarity by considering only a double cut through one quark and one gluon propagator, we find the same color decomposition if we consider cuts of one-loop amplitudes through two gluon lines or two quark lines.

\subsection{Amplitudes with multiple quark pairs}

As we explained in the previous subsections, it may be beneficial to employ unitary color decomposition in one-loop computations. Such decomposition is known for a $n$-gluon and $\bar{q} q+n$-gluon scattering amplitudes, but not for scattering amplitudes that involve multiple quark pairs. For this reason, amplitudes with multiple quark pairs represent a special case. Indeed, it is easy to understand how such amplitudes can be written in terms of color-ordered amplitudes and how primitive amplitudes can be constructed, but it is not straightforward to connect the color-ordered and primitive amplitudes. Below we explain how the relationship between color-ordered and primitive amplitudes can be established considering a simple process $0 \rightarrow \bar{q} q \bar{Q} Q g$.

We begin by discussing the decomposition of full scattering amplitudes into color-ordered amplitudes for $m$ non-identical quark pairs. The total number of quarks and anti-quarks is $n=2 \mathrm{~m}$. Because quark lines are connected by gluon lines, we can write a color factor associated with a particular quark line $m_{1}$ as a matrix element of a product of certain number of $T$-matrices, taken between quark and anti-quark color states $\left(T^{a_{1}} T^{a_{2}} \ldots T^{a_{r}}\right)_{i_{2 m_{1}} \bar{z}_{2 m_{1}-1}}$. The adjoint labels $\left(a_{1}, \ldots, a_{r}\right)$ are internal indices; they are contracted with similar indices carried by other quark lines or with the adjoint indices that enter three- and four-gluon vertices. All these contractions can be turned to products of Kronecker deltasymbols with repeated use of the identity

$$
\left(T^{a}\right)_{i_{2} \bar{\imath}_{1}}\left(T^{a}\right)_{i_{4} \bar{\imath}_{3}}=\delta_{i_{2} \bar{\imath}_{3}} \delta_{i_{4} \bar{\imath}_{1}}-\frac{1}{N_{c}} \delta_{i_{2} \bar{\imath}_{1}} \delta_{i_{4} \bar{\imath}_{3}} .
$$

It follows from Eq. (6.51) that, to leading order in $N_{c}$, the color flow of antiquarks is flipped. The sub-leading term in $N_{c}$ ensures that the decomposition respects the tracelessness of $T$-matrices. Because two quark lines can only be connected by one gluon line, we conclude that a convenient basis for the color decomposition is given by the set of Kronecker deltas

$$
C\left(\sigma, r_{\sigma}\right)=\left\{\frac{(-1)^{r_{\sigma}}}{N_{c}^{r_{\sigma}}} \delta_{i_{2} \bar{i}_{(2 \sigma(1)-1)}} \delta_{i_{4} \bar{\imath}(2 \sigma(2)-1)} \ldots \delta_{i_{2 m} \bar{\imath}_{2 \sigma(m)-1}}\right\},
$$


where $\sigma$ denotes permutations of $m$ anti-quark indices and $r_{\sigma}$ is the rank of the permutation $\sigma$. The rank is computed by counting how many times the equation $\sigma(k)=k, k=1 . . m$, is satisfied for a given permutation $\sigma$. If $\{\sigma(k)\}=\{k\}$ for all $k, r_{\sigma}=m-1$. At one loop the basis remains the same, except for an overall factor of $N_{c}$. Finally, we note that we can describe scattering amplitudes with $m$-quark pairs and $n-2 m$ gluons by replacing Kronecker delta-symbols in Eq. (6.52) with products of $T$ matrices. This leads to a new basis set

$$
C\left(\sigma, r_{\sigma},\left\{n_{i}\right\}\right)=\left\{\frac{(-1)^{r}}{N_{c}^{r_{\sigma}}}\left(T^{a_{1}: a_{n_{1}}}\right)_{i_{2} \bar{i}_{\sigma_{1}}} \ldots\left(T^{a_{n_{m-1}}: a_{n_{m}}}\right)_{i_{2 m} \bar{i}_{\sigma m}}\right\},
$$

where we use the notation $\sigma_{k}=2 \sigma(k)-1$ and $T^{a_{n_{1}}: a_{n_{2}}}=T^{a_{n_{1}}} \ldots . T^{a_{n_{2}}}$. In addition, the set $\left\{n_{i}\right\}, i=1, \ldots, m$, contains all possible partitions of $n-2 m$ gluons into $m$ subsets. We do not pursue the general color-decomposition discussion in what follows and turn, instead, to an example.

As clearly follows from the above discussion, the color-decomposition of amplitudes with multiple fermion pairs can be performed in a straightforward way. However, within the unitarity framework we compute primitive, rather than color-ordered, amplitudes. We can use color-stripped Feynman rules and the fact that all particles are ordered in a given primitive amplitude, to construct the primitive amplitudes directly. The non-trivial step is to connect the primitive amplitudes, so constructed, with color-ordered or full amplitudes. We will explain how to do that by considering the process $0 \rightarrow \bar{q} q \bar{Q} Q g$. Applying the general results discussed above, we find the color decomposition for tree and one-loop amplitudes

$$
\begin{gathered}
\mathcal{B}^{\text {tree }}\left(\bar{q}_{1}, q_{2}, \bar{Q}_{3}, Q_{4}, g_{5}\right)=g_{s}^{3}\left[\left(T^{a_{5}}\right)_{i_{4} \bar{\imath}_{1}} \delta_{i_{2} \bar{\tau}_{3}} B_{5 ; 1}^{\text {tree }}+\frac{1}{N_{c}}\left(T^{a_{5}}\right)_{i_{2} \bar{\imath}_{1}} \delta_{i_{4} \bar{\imath}_{3}} B_{5 ; 2}^{\text {tree }}\right. \\
\left.+\left(T^{a_{5}}\right)_{i_{2} \bar{\tau}_{3}} \delta_{i_{4} \bar{\imath}_{1}} B_{5 ; 3}^{\text {tree }}+\frac{1}{N_{c}}\left(T^{a_{5}}\right)_{i_{4} \bar{\tau}_{3}} \delta_{i_{2} \bar{\imath}_{1}} B_{5 ; 4}^{\text {tree }}\right] \\
\mathcal{B}^{1-\text { loop }}\left(\bar{q}_{1}, q_{2}, \bar{Q}_{3}, Q_{4}, g_{5}\right)=g_{s}^{5}\left[N_{c}\left(T^{a_{5}}\right)_{i_{4} \bar{\imath}_{1}} \delta_{i_{2} \bar{\imath}_{3}} B_{5 ; 1}+\left(T^{a_{5}}\right)_{i_{2} \bar{\imath}_{1}} \delta_{i_{4} \bar{\imath}_{3}} B_{5 ; 2}\right. \\
\left.+N_{c}\left(T^{a_{5}}\right)_{i_{2} \bar{\tau}_{3}} \delta_{i_{4} \bar{\imath}_{1}} B_{5 ; 3}+\left(T^{a_{5}}\right)_{i_{4} \bar{\imath}_{3}} \delta_{i_{2} \bar{\imath}_{1}} B_{5 ; 4}\right]=\sum_{i=1}^{4} C_{i} B_{5 ; i},
\end{gathered}
$$

where we introduced the notation $C_{i}$ for the elements of the color basis. Each of these one-loop color-ordered amplitudes can be written as a sum of two terms

$$
B_{5 ; i}=B_{5 ; i}^{[1]}+\frac{n_{f}}{N_{c}} B_{5 ; i}^{[1 / 2]}, \quad i=1,2,3,4,
$$

to separate diagrams with a closed fermion loop from the other ones. The amplitudes $B_{5 ; i}^{[1]}$ and $B_{5 ; i}^{[1 / 2]}$ can be expressed through linear combinations of primitive amplitudes. We will explicitly show how to do that for amplitudes with a closed fermion loop $B_{5 ; i}^{[1 / 2]}$. 


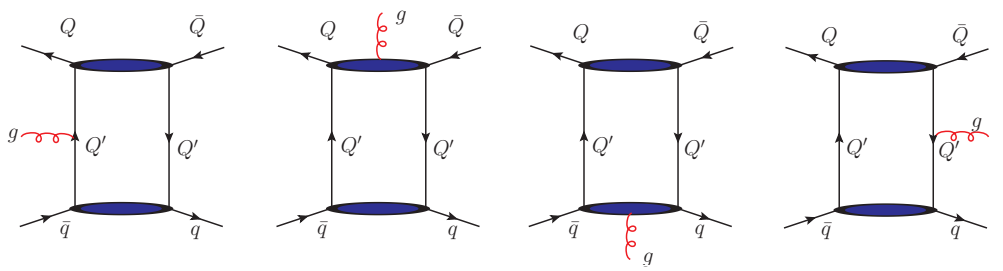

Figure 6.3: Prototypes of four parent diagrams that define the primitive amplitudes for the $n_{f}$ part of the $0 \rightarrow \bar{q} q \bar{Q} Q g$ amplitude. The solid blobs denote "dummy lines" described in the text.

Before proceeding with this discussion, we explain the general strategy. Since particles in each primitive amplitude are ordered, primitive amplitudes can be characterized by "parent diagrams". A parent diagram for a primitive amplitude is the diagram with the ordering of external particles consistent with the ordering of external particles in the primitive amplitude and the maximal number of propagators that depend on the loop momenta. The primitive amplitudes can be constructed from the parent diagrams by pinching internal propagators and resolving illegitimate vertices by creating propagators that do not depend on the loop momentum. Since pinching and pulling does not change the ordering of external particles, every diagram obtained by this procedure contributes to the same primitive amplitude. Therefore, if we take a Feynman diagram, we can study its color factor to find the color-ordered amplitudes that it contributes to. On the other hand, since the "pinching and pulling" technique connects primitive amplitudes with the color-stripped Feynman diagrams, we can find primitive amplitudes that receive contributions from a particular Feynman diagram. Because color-ordered amplitudes are given by linear combinations of primitive amplitudes with coefficients that only depend on the number of colors $N_{c}$, it is sufficient to analyze a number of Feynman diagrams to establish the connection uniquely.

We now illustrate this general strategy by considering amplitudes with a closed fermion loop $B_{5 ; i}^{[1 / 2]}$. Since the maximal number of propagators in the loops that contribute to $0 \rightarrow q \bar{q} Q \bar{Q} g$ is five but the fermion loop contribution has at most three propagators, we find it to be convenient to introduce dummy lines, to get a uniform graphical representation of the primitive amplitudes. A dummy line represents a propagator that is not part of the loop. Contributions obtained by cutting dummy lines vanish. There are four primitive amplitudes $A_{i}^{[1 / 2]}$, that are obtained if a gluon is inserted in different ways between four quark lines. They are shown in Fig. 6.3. We introduce the notation

$$
\begin{array}{ll}
A_{1}^{[1 / 2]}=A_{L}^{[1 / 2]}\left(\bar{q}_{1}, g_{5}, Q_{4}, \bar{Q}_{3}, q_{2}\right), & A_{2}^{[1 / 2]}=A_{L}^{[1 / 2]}\left(\bar{q}_{1}, Q_{4}, \bar{Q}_{3}, q_{2}, g_{5}\right), \\
A_{3}^{[1 / 2]}=A_{L}^{[1 / 2]}\left(\bar{q}_{1}, Q_{4}, \bar{Q}_{3}, g_{5}, q_{2}\right), & A_{4}^{[1 / 2]}=A_{L}^{[1 / 2]}\left(\bar{q}_{1}, Q_{4}, g_{5}, \bar{Q}_{3}, q_{2}\right) .
\end{array}
$$

Because of Furry's theorem, we have only three independent primitive ampli- 


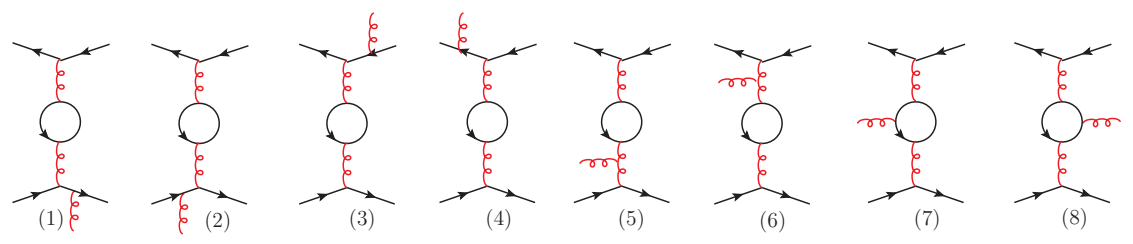

Figure 6.4: Eight diagrams that contribute to the $n_{f}$ part of the $0 \rightarrow \bar{q} q \bar{Q} Q g$ amplitude.

tudes

$$
\sum_{i=1}^{4} A_{i}^{[1 / 2]}=0
$$

In general, the color-ordered amplitudes are given by linear combinations of primitive amplitudes

$$
B_{5 ; i}^{[1 / 2]}=\sum_{j=1}^{4} x_{i j} A_{j}^{[1 / 2]} .
$$

If the primitive amplitudes are represented by diagrams with dummy lines, then the same primitive amplitude can have more than one equivalent parent Feynman-diagram. They have the same ordering and can be transformed into each other by pulling and pinching propagators as explained above. In our case we have eight Feynman diagrams shown in Fig. 6.4. Each diagram is factorized into a color factor and a color-stripped part. The colorless diagrams with all vertices oriented clockwise are obtained by setting the color matrices for quarks and gluons to -1 . The relations between the color-ordered amplitudes and the primitive amplitudes are established by expanding the color factors of the parent diagrams in the color basis of Eq. (6.55). Since we have only three independent primitive amplitudes we need to choose three independent parent diagrams and trace how they are mapped onto color-ordered amplitudes. For the sake of definiteness, we choose the first three diagrams of Fig. 6.4 and write

$$
\mathcal{B}_{n_{f}}^{1 \text {-loop }} \mid=N_{c}^{-1} \sum_{i=1}^{4} C_{i} B_{5 ; i}^{[1 / 2]}=\sum_{i=1}^{3} \operatorname{Col}_{i} D_{i}+\ldots
$$

where the ellipsis stands for contributions of diagrams four to eight. The decomposition of the color factors of these diagrams into the color basis reads

$$
\begin{aligned}
& \mathrm{Col}_{1}=\left(T^{a} T^{x}\right)_{i_{2} \bar{\imath}_{1}}\left(T^{x}\right)_{i_{4} \bar{\imath}_{3}}=N_{c}^{-1}\left(C_{3}-C_{2}\right), \\
& \mathrm{Col}_{2}=\left(T^{x} T^{a}\right)_{i_{2} \bar{\imath}_{1}}\left(T^{x}\right)_{i_{4} \bar{\imath}_{3}}=N_{c}^{-1}\left(C_{1}-C_{2}\right), \\
& \mathrm{Col}_{3}=\left(T^{x} T^{a}\right)_{i_{4} \bar{\imath}_{3}}\left(T^{x}\right)_{i_{2} \bar{\imath}_{1}}=N_{c}^{-1}\left(C_{3}-C_{4}\right) .
\end{aligned}
$$

The primitive amplitudes are given by linear combinations of colorless ordered parent diagrams. Using color-stripped Feynman rules, we find

$$
A_{1}^{[1 / 2]}=-D_{2}+\ldots, \quad A_{2}^{[1 / 2]}=D_{1}+D_{2} \ldots, \quad A_{3}^{[1 / 2]}=-D_{1}-D_{3} \ldots,
$$




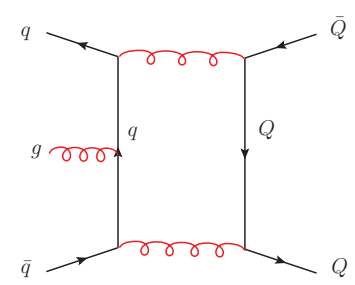

(a)

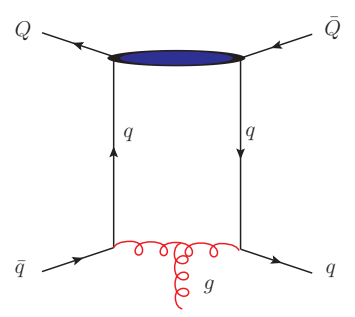

(b)

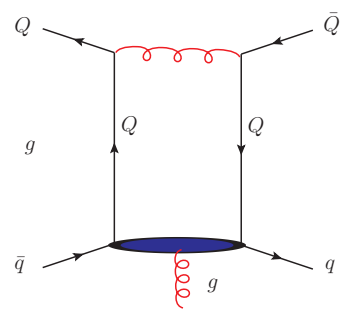

(c)

Figure 6.5: Prototype of parent diagrams for primitive amplitudes with four quarks and one gluon. The gluons can be inserted in four possible ways into the prototype diagrams, leading to four primitive amplitudes. The solid blobs denote dummy lines described this section.

where ellipsis stand for the contributions of diagrams four to eight. We derive the inverse relations

$$
D_{2}=-A_{1}^{[1 / 2]}, \quad D_{1}=A_{1}^{[1 / 2]}+A_{2}^{[1 / 2]}, \quad D_{3}=-A_{1}^{[1 / 2]}-A_{2}^{[1 / 2]}-A_{3}^{[1 / 2]}
$$

and use Eqs. (6.61), (6.63) and (6.58) to get

$$
\mathcal{B}_{n_{f}}^{1 \text {-loop }}=N_{c}^{-1} \sum_{i=1}^{4} C_{i} B_{5 ; i}^{[1 / 2]}=-N_{c}^{-1} \sum_{i=1}^{4} C_{i} A_{i}^{[1 / 2]} .
$$

Comparing Eq. (6.64) with Eq. (6.59), we conclude that $x_{i j}=-\delta_{i j}$. Explicitly, the relation between color-ordered and primitive amplitudes reads

$$
\begin{aligned}
& B_{5 ; 1}^{[1 / 2]}=-A_{L}^{[1 / 2]}\left(\bar{q}_{1}, g_{5}, Q_{4}, \bar{Q}_{3}, q_{2}\right), \\
& B_{5 ; 2}^{[1 / 2]}=-A_{L}^{[1 / 2]}\left(\bar{q}_{1}, Q_{4}, \bar{Q}_{3}, q_{2}, g_{5}\right), \\
& B_{5 ; 3}^{[1 / 2]}=-A_{L}^{[1 / 2]}\left(\bar{q}_{1}, Q_{4}, \bar{Q}_{3}, g_{5}, q_{2}\right), \\
& B_{5 ; 4}^{[1 / 2]}=-A_{L}^{[1 / 2]}\left(\bar{q}_{1}, Q_{4}, g_{5}, \bar{Q}_{3}, q_{2}\right) .
\end{aligned}
$$

We note that our derivation relies on a particular choice of parent diagrams for primitive amplitudes but it is straightforward to check that the final result is independent of this choice.

The $n_{f}$-independent part of the amplitudes $B_{5 ; i}^{[1]}$ have contributions of three classes of primitive amplitudes as indicated in Fig. 6.5. Following the same procedure as in the case of the $n_{f}$-dependent part, we can work out relations between the color-ordered amplitudes for each class and the corresponding primitive amplitudes. Such relations can be found in Ref. [67] and we do not repeat them here.

Finally, we note that the decomposition of color-ordered amplitudes in terms of primitive amplitudes becomes more and more cumbersome as the number of quark pairs increases. Partially motivated by this difficulty a different approach 
was suggested in Ref. [94]. The starting point is the observation that, to implement unitarity, complete ordering is not necessary. We only need to consider all partitions of the external particles into subsets of particles that can be connected to cut propagators in the loop. The particles within a given subset are not ordered. In addition, the color degrees of freedom are treated at the same footing as other quantum numbers. This treatment is facilitated by the use of the color-flow representation and the color-dressed Berends-Giele recursion relations [95]. By considering gluon scattering amplitudes it was shown in Ref. [94] that scaling of the computer time and the stability of the numerical evaluation is competitive with the implementation based on ordered approach. To understand merits of this implementation, further work is required.

\subsection{Singular behavior of one-loop amplitudes}

In this Section we discuss the singular behavior of one-loop amplitudes $[96,97]$. Although this topic is interesting in its own right, the reason we present it here is a particular representation of color algebra employed in Ref. [97] to present singular limits of one-loop amplitudes in a simple way. This representation of the color algebra has the advantage that it treats the color degrees of freedom of quarks and gluons on the same footing. Below we explain how to connect this representation with more conventional treatment of color degrees of freedom presented above.

The infrared singular behavior of one-loop amplitudes is completely known, specifically in the case when the singularities are regularized by dimensional regularization. Although the singular terms do not in the end contribute to physical answers, they provide an important check on the correctness of intermediate results. This check can be performed both when setting up an analytic formula, and also when performing numerical evaluation of the answer, to control the numerical stability, see Section 10.2.

The results for the infrared-singular behaviour of on-shell QCD amplitudes at one- and two-loop orders with massless particles have been given in Ref. [97]. The generalization to the case when massive particles are present is given at oneloop order in Ref. [98]. In this Section we shall consider only the case where there are no massive partons and refer the reader to Ref. [98] for more the complicated case. The results of Ref. [97] are presented using a color space notation, which has the advantage that it is very compact and can deal with both quarks and gluons in a seamless way. However, this notation is not particularly well-known and, in what follows, we give several examples to elucidate the notation and connect it to the discussion of color given earlier in this Section.

We begin by writing the perturbative expansion of scattering amplitudes following Ref. [97]

$$
|\mathcal{M}\rangle=g_{s}^{r}\left(\left|\mathcal{M}^{(0)}(\{p, \lambda\})\right\rangle+g_{s}^{2} c_{\Gamma}\left|\mathcal{M}^{(1)}\left(\mu^{2} ;\{p, \lambda\}\right)\right\rangle+\ldots\right),
$$

where $r$ is the overall power of the (unrenormalized) strong coupling constant $g_{s}$ and $\{p, \lambda\}$ is a set of momenta and helicities of the external particles. The amplitude is written using a bra and ket notation, to indicate that it is a vector 
in color space. The one-loop subamplitude $\left|\mathcal{M}^{(1)}\left(\mu^{2} ;\{p\}\right)\right\rangle$ has both double and single poles in $1 / \epsilon$. Catani's result [97] is that these singularities can be obtained from the tree amplitudes by operating with the color-space operator $\boldsymbol{I}^{(1)}$,

$$
\left|\mathcal{M}^{(1)}\left(\mu^{2} ;\{p, \lambda\}\right)\right\rangle=\boldsymbol{I}^{(1)}\left(\epsilon, \mu^{2} ;\{p\}\right)\left|\mathcal{M}^{(0)}(\{p, \lambda\})\right\rangle+O\left(\epsilon^{0}\right) .
$$

The operator $\boldsymbol{I}^{(1)}$ has the following explicit expression in terms of color charges of the external particles

$$
\boldsymbol{I}^{(1)}\left(\epsilon, \mu^{2} ;\{p\}\right)=\frac{r}{2} \frac{\beta_{0}}{\epsilon}+\sum_{i} \frac{1}{\boldsymbol{T}_{i}^{2}} \mathcal{V}_{i}^{\mathrm{sing}}(\epsilon) \sum_{j \neq i} \boldsymbol{T}_{i} \cdot \boldsymbol{T}_{j}\left(\frac{\mu^{2}}{-s_{i j}-i 0}\right)^{\epsilon},
$$

where $s_{i j}=2 p_{i} \cdot p_{j}, \beta_{0}=11 / 3 C_{A}-2 / 3 T_{R} N_{f}$ is the first coefficient in the expansion of the QCD $\beta$-function. The singular function $\mathcal{V}_{i}^{\text {sing }}(\epsilon)$ depends only on the parton type $i$ and is given by

$$
\mathcal{V}_{i}^{\text {sing }}(\epsilon)=\frac{\boldsymbol{T}_{i}^{2}}{\epsilon^{2}}+\frac{\gamma_{i}}{\epsilon}
$$

Squares of the color-charge operators $\boldsymbol{T}_{i}^{2}$ and coefficients $\gamma_{i}$ for quarks and gluons are

$$
\begin{aligned}
\boldsymbol{T}_{q}^{2}=\boldsymbol{T}_{\bar{q}}^{2}=C_{F}, & \boldsymbol{T}_{g}^{2}=C_{A}, \\
\gamma_{q}=\gamma_{\bar{q}}=\frac{3}{2} C_{F}, & \gamma_{g}=\frac{\beta_{0}}{2} .
\end{aligned}
$$

We note that in Eq. (6.67) the double poles in $\epsilon$ are factorized completely. If we expand Eq. (6.68) in powers of $\epsilon$ and then use the color conservation relation, $\sum_{j \neq i} \boldsymbol{T}_{j}=-\boldsymbol{T}_{i}$, we obtain the result

$$
\boldsymbol{I}^{(1)}\left(\epsilon, \mu^{2} ;\{p\}\right)=\sum_{i} \frac{1}{\epsilon^{2}} \sum_{j \neq i} \boldsymbol{T}_{i} \cdot \boldsymbol{T}_{j}+\mathcal{O}\left(\frac{1}{\epsilon}\right)=-\frac{1}{\epsilon^{2}} \sum_{i} \boldsymbol{T}_{i}^{2}+\mathcal{O}\left(\frac{1}{\epsilon}\right),
$$

that explicitly shows the absence of color correlations at $\mathcal{O}\left(1 / \epsilon^{2}\right)$. Note however that the single $1 / \epsilon$ poles have color correlations. We will explicitly show how to compute those by considering a few examples below.

\section{Divergence structure of the one-loop $\bar{q} q g g$ amplitude}

As a first example we compute the singular behavior of the one-loop $\bar{q} q g g$ amplitude, which illustrates how color operators act on both quark and gluon fields. Here we shall present the singularity structure of primitive amplitudes, since primitive amplitudes are the building blocks in one-loop calculations. To arrive at the divergent structure of the primitive amplitudes, we will write the divergences of the one-loop $\bar{q} q g g$ amplitude using the color basis shown in Eq. (6.50). We begin by acting with the color vector on the tree amplitude. We recover the 
regular color-ordered amplitudes for a particular choice of quark and antiquark color indices, $i_{2}, \bar{l}_{1}$ and gluon indices, $a_{3}, a_{4}$

$$
\begin{aligned}
\left|\mathcal{M}^{(0)}\right\rangle & =\left|\mathcal{M}_{34}^{(0)}\right\rangle+\left|\mathcal{M}_{43}^{(0)}\right\rangle, \\
\left\langle\bar{\imath}_{1} i_{2} a_{3} a_{4} \mid \mathcal{M}_{34}^{(0)}\right\rangle & =\left(T^{a_{3}} T^{a_{4}}\right)_{i_{2} \bar{\imath}_{1}} m_{4}\left(\bar{q}_{1}, q_{2}, g_{3}, g_{4}\right),
\end{aligned}
$$

where $m_{4}\left(\bar{q}_{1}, q_{2}, g_{3}, g_{4}\right)$ is the tree color-stripped amplitude. The tree-graph amplitude for $\bar{q} q g g$ scattering is proportional to the second power of the strong coupling constant, so that $r$ as defined in Eq. (6.66) is equal to two. As a result the contributions of $\beta_{0}$ and $\gamma_{g}$ in Eq. (6.68) cancel. This leads to the following structure for the infrared poles

$$
\begin{aligned}
\left\langle\overline{1}_{1} i_{2} a_{3} a_{4} \mid \mathcal{M}^{(1)}\right\rangle= & \sum_{k} \sum_{n \neq k}\left(\frac{1}{\epsilon^{2}}+\frac{1}{\epsilon}\left(\frac{\gamma_{q}}{T_{q}^{2}}\left(\delta_{k 1}+\delta_{k 2}\right)+L_{k n}\right)\right) \\
& \times\left\langle\bar{\imath}_{1} i_{2} a_{3} a_{4}\left|\boldsymbol{T}_{k} \cdot \boldsymbol{T}_{n}\right| \mathcal{M}^{(0)}\right\rangle,
\end{aligned}
$$

where we have introduced the notation $L_{k n}=\ln \left(\mu^{2} /\left(-s_{k n}-i 0\right)\right)$. To calculate the matrix element of the product of two color charge operators between a state of definite color and the amplitude $\left|\mathcal{M}^{(0)}\right\rangle$, we insert a complete set of color states

$$
\begin{aligned}
\left\langle\bar{\imath}_{1} i_{2} a_{3} a_{4}\left|\boldsymbol{T}_{k} \cdot \boldsymbol{T}_{n}\right| \mathcal{M}^{(0)}\right\rangle & =\sum_{\mathcal{P}(3,4)}\left\langle\bar{\imath}_{1} i_{2} a_{3} a_{4}\left|\boldsymbol{T}_{k} \cdot \boldsymbol{T}_{n}\right| \mathcal{M}_{\sigma(3), \sigma(4)}^{(0)}\right\rangle \\
\left\langle\bar{\imath}_{1} i_{2} a_{3} a_{4}\left|\boldsymbol{T}_{k} \cdot \boldsymbol{T}_{n}\right| \mathcal{M}_{34}^{(0)}\right\rangle & =\sum_{\kappa}\left\langle\bar{l}_{1} i_{2} a_{3} a_{4}\left|\boldsymbol{T}_{k} \cdot \boldsymbol{T}_{n}\right| \kappa\right\rangle\left\langle\kappa \mid \mathcal{M}_{34}^{(0)}\right\rangle
\end{aligned}
$$

where we use the notation $|\kappa\rangle=\left|\bar{\jmath}_{1} j_{2} b_{3} b_{4}\right\rangle$. We can evaluate the matrix elements of the color charge operators using the following matrix elements $\left\langle i_{k}\left|\boldsymbol{T}_{k}^{x}\right| j_{k}\right\rangle=$ $T_{i_{k} j_{k}}^{x} / \sqrt{2}$ if $k$ is a quark, $\left\langle\bar{\lambda}_{k}\left|\boldsymbol{T}_{k}^{x}\right| \bar{\jmath}_{k}\right\rangle=-T_{\bar{\jmath}_{k} \bar{\imath}_{k}}^{x} / \sqrt{2}$ if $k$ is an-antiquark, and $\left\langle a_{k}\left|\boldsymbol{T}_{k}^{x}\right| b_{k}\right\rangle=F_{a_{k} b_{k}}^{x} / \sqrt{2}$ if $k$ is a gluon. Also, we note that the color-charge operator $\boldsymbol{T}_{k}$ only acts on the color index of a parton $k$.

We now write down the color correlation matrix elements for $q \bar{q}, g g, q g$ and $\bar{q} g$ and bring those color structures to a form consistent with Eq. (6.50). For the $\bar{q} q g g$ case, this implies that the result should be expressed through the following color structures

$$
\begin{gathered}
\left(T^{x_{1}} T^{x_{2}}\right)_{i_{2} \bar{\imath}_{1}}\left(F^{a_{4}} F^{a_{1}}\right)_{x_{1} x_{2}}, \quad\left(T^{x_{1}} T^{a_{3}} T^{x_{2}}\right)_{i_{2} \bar{\imath}_{1}}\left(F^{a_{4}}\right)_{x_{1} x_{2}}, \\
\left(T^{x_{1}} T^{a_{3}} T^{a_{4}} T^{x_{2}}\right)_{i_{2} \bar{\nu}_{1}} \delta_{x_{1} x_{2}},
\end{gathered}
$$

where the summation over repeated indices is assumed and $3 \leftrightarrow 4$ permutations are not shown.

We begin with the $q g_{3}$ case. The matrix element reads

$$
\left\langle\bar{\imath}_{1} i_{2} a_{3} a_{4}\left|\boldsymbol{T}_{2} \cdot \boldsymbol{T}_{3}\right| \bar{\jmath}_{1} j_{2} b_{3} b_{4}\right\rangle=\frac{1}{2}\left(T^{x}\right)_{i_{2} j_{2}}\left(F^{x}\right)_{a_{3} b_{3}} \delta_{a_{4} b_{4}} \delta_{\bar{\imath}_{1} \bar{\jmath}_{1}} .
$$


This implies

$$
\left\langle\bar{\imath}_{1} i_{2} a_{3} a_{4}\left|\boldsymbol{T}_{2} \cdot \boldsymbol{T}_{3}\right| \mathcal{M}_{34}^{(0)}\right\rangle=\frac{1}{2}\left(F^{x}\right)_{a_{3} b_{3}}\left(T^{x} T^{b_{3}} T^{a_{4}}\right)_{i_{2} \bar{\imath}_{1}} m_{4}\left(\bar{q}_{1}, q_{2}, g_{3}, g_{4}\right) .
$$

The color factor in the above equation is not yet in the right form (c.f. Eq. (6.75)). We therefore commute $T^{b_{3}}$ to the right using $\left[T^{a}, T^{b}\right]=-F_{b y}^{a} T^{y}$ and obtain

$$
F_{a_{3} b_{3}}^{x}\left(T^{x} T^{b_{3}} T^{a_{4}}\right)_{i_{2} \bar{\imath}_{1}}=-F_{x b_{3}}^{a_{3}}\left(T^{x} T^{a_{4}} T^{b_{3}}\right)_{i_{2} \bar{\nu}_{1}}-\left(F^{a_{3}} F^{a_{4}}\right)_{x y}\left(T^{x} T^{y}\right)_{i_{2} \bar{\nu}_{1}} .
$$

This leads to

$$
\begin{aligned}
& \left\langle\bar{\imath}_{1} i_{2} a_{3} a_{4}\left|\boldsymbol{T}_{2} \cdot \boldsymbol{T}_{3}\right| \mathcal{M}_{34}^{(0)}\right\rangle=-\frac{1}{2}\left[\left(T^{x} T^{a_{4}} T^{y}\right)_{i_{2} \bar{\imath}_{1}}\left(F^{a_{3}}\right)_{x y}\right. \\
& \left.+\left(T^{x} T^{y}\right)_{i_{2} \bar{\imath}_{1}}\left(F^{a_{3}} F^{a_{4}}\right)_{x y}\right] m_{4}\left(\bar{q}_{1}, q_{2}, g_{3}, g_{4}\right) .
\end{aligned}
$$

On the other hand, considering the color correlation $q g_{4}$ we find

$$
\left\langle\bar{\imath}_{1} i_{2} a_{3} a_{4}\left|\boldsymbol{T}_{2} \cdot \boldsymbol{T}_{4}\right| \bar{\jmath}_{1} j_{2} b_{3} b_{4}\right\rangle=\frac{1}{2}\left(T^{x}\right)_{i_{2} j_{2}}\left(F^{x}\right)_{a_{4} b_{4}} \delta_{a_{3} b_{3}} \delta_{\bar{\imath}_{1} \bar{\jmath}_{1}},
$$

so that color factors are already in the right form

$$
\left\langle\bar{\imath}_{1} i_{2} a_{3} a_{4}\left|\boldsymbol{T}_{2} \cdot \boldsymbol{T}_{4}\right| \mathcal{M}_{34}^{(0)}\right\rangle=-\frac{1}{2}\left(F^{a_{4}}\right)_{x b_{4}}\left(T^{x} T^{a_{3}} T^{b_{4}}\right)_{i_{2} \bar{\imath}_{1}} m_{4}\left(\bar{q}_{1}, q_{2}, g_{3}, g_{4}\right) .
$$

Similarly for the case $\boldsymbol{T}_{2} \cdot \boldsymbol{T}_{1}$ we have

$$
\left\langle\bar{\imath}_{1} i_{2} a_{3} a_{4}\left|\boldsymbol{T}_{2} \cdot \boldsymbol{T}_{1}\right| \bar{\jmath}_{1} j_{2} b_{3} b_{4}\right\rangle=-\frac{1}{2}\left(T^{x}\right)_{i_{2} j_{2}} \delta_{a_{3} b_{3}} \delta_{a_{4} b_{4}}\left(T^{x}\right)_{\bar{\jmath}_{1} \bar{\imath}_{1}}
$$

so that

$$
\left\langle\bar{\imath}_{1} i_{2} a_{3} a_{4}\left|\boldsymbol{T}_{2} \cdot \boldsymbol{T}_{1}\right| \mathcal{M}_{34}^{(0)}\right\rangle=-\frac{1}{2}\left(T^{x} T^{a_{3}} T^{a_{4}} T^{x}\right)_{i_{2} \bar{\imath}_{1}} m_{4}\left(\bar{q}_{1}, q_{2}, g_{3}, g_{4}\right) .
$$

Evaluating the remaining matrix elements we obtain

$$
\begin{gathered}
\left\langle\bar{\imath}_{1} i_{2} a_{3} a_{4}\left|\boldsymbol{T}_{3} \cdot \boldsymbol{T}_{4}\right| \mathcal{M}_{34}^{(0)}\right\rangle=-\frac{1}{2}\left(T^{x} T^{y}\right)_{i_{2} \bar{\imath}_{1}}\left(F^{a_{3}} F^{a_{4}}\right)_{x y} m_{4}\left(\bar{q}_{1}, q_{2}, g_{3}, g_{4}\right), \\
\left\langle\bar{\imath}_{1} i_{2} a_{3} a_{4}\left|\boldsymbol{T}_{3} \cdot \boldsymbol{T}_{1}\right| \mathcal{M}_{34}^{(0)}\right\rangle=-\frac{1}{2}\left(T^{x} T^{a_{4}} T^{y}\right)_{i_{2} \bar{\tau}_{1}}\left(F^{a_{3}}\right)_{x y} m_{4}\left(\bar{q}_{1}, q_{2}, g_{3}, g_{4}\right), \\
\left\langle\overline{1}_{1} i_{2} a_{3} a_{4}\left|\boldsymbol{T}_{4} \cdot \boldsymbol{T}_{1}\right| \mathcal{M}_{34}^{(0)}\right\rangle=-\frac{1}{2}\left[\left(T^{x} T^{a_{3}} T^{y}\right)_{i_{2} \bar{\tau}_{1}}\left(F^{a_{4}}\right)_{x y}\right. \\
\left.+\left(T^{x} T^{y}\right)_{i_{2} \bar{\imath}_{1}}\left(F^{a_{3}} F^{a_{4}}\right)_{x y}\right] m_{4}\left(\bar{q}_{1}, q_{2}, g_{3}, g_{4}\right) .
\end{gathered}
$$


Finally, putting everything together we find

$$
\begin{aligned}
& \left\langle i_{2} a_{3} a_{4} \bar{\imath}_{1} \mid \mathcal{M}^{(1)}\right\rangle=-\sum_{P(3,4)} m_{4}\left(\bar{q}_{1}, q_{2}, g_{3}, g_{4}\right)[ \\
& \left(T^{x_{1}} T^{x_{2}}\right)_{i_{2} \bar{\imath}_{1}}\left(F^{a_{3}} F^{a_{4}}\right)_{x_{1} x_{2}}\left(\frac{3}{\epsilon^{2}}+\frac{1}{\epsilon}\left(\frac{3}{2}+\sum_{i=2,4} L_{i, i+1}\right)\right) \\
& +\left(T^{x_{1}} T^{a_{4}} T^{x_{2}}\right)_{i_{2} \bar{\imath}_{1}}\left(F^{a_{3}}\right)_{x_{1} x_{2}}\left(\frac{2}{\epsilon^{2}}+\frac{1}{\epsilon}\left(\frac{3}{2}+L_{23}+L_{13}\right)\right) \\
& +\left(T^{x_{1}} T^{a_{3}} T^{x_{2}}\right)_{i_{2} \bar{\imath}_{1}}\left(F^{a_{4}}\right)_{x_{1} x_{2}}\left(\frac{2}{\epsilon^{2}}+\frac{1}{\epsilon}\left(\frac{3}{2}+L_{13}+L_{14}\right)\right) \\
& \left.+\left(T^{x_{1}} T^{a_{3}} T^{a_{4}} T^{x_{1}}\right)_{i_{2} \bar{\imath}_{1}}\left(\frac{1}{\epsilon^{2}}+\frac{1}{\epsilon}\left(\frac{3}{2}+L_{12}\right)\right)\right]+\mathcal{O}\left(\epsilon^{0}\right) .
\end{aligned}
$$

It follows from Eq. (6.50) that each of the color structures in Eq. (6.85) is uniquely associated with the primitive amplitudes for the $\bar{q} q g g$ process. In particular, the structure of the poles of primitive amplitudes that appears in Eq. (6.85) is remarkably simple: the coefficient of the double pole is equal to the number of gluon propagators in the parent diagram of the given primitive. The single pole contains, beyond the terms $\gamma_{q} / C_{F}$ associated with the quark line, only logarithms of scalar products of pairs of parton momenta, where the relevant pairs of momenta are connected by gluon propagators in the parent diagram. The generalization of this result to the case of primitive amplitudes for the $\bar{q} q+(n-2)$-gluon case reads

$$
\begin{gathered}
\tilde{m}_{n}^{(1)}\left(\bar{q}_{n}, g_{k+1}, \ldots, g_{n-1}, q_{2}, g_{3}, \ldots g_{k}\right)=\tilde{m}_{n}\left(\bar{q}_{n}, g_{k+1}, \ldots, g_{n-1}, q_{2}, g_{3}, \ldots g_{k}\right) \\
\times\left[-\frac{k}{\epsilon^{2}}-\frac{1}{\epsilon}\left(\frac{3}{2}+\sum_{i=1}^{k-1} L_{i i+1}+L_{k n}\right)\right]+\mathcal{O}\left(\epsilon^{0}\right),
\end{gathered}
$$

where the tree primitive amplitudes $\tilde{m}_{n}$ are defined in Eq. (6.45). In the following subsection we derive the corresponding result for pure gluon amplitudes for all values of $n$.

Divergence structure of n-gluon amplitudes

As our second example we use Eq. (6.68) to derive the divergences of the primitive amplitudes for an $n$-gluon scattering, neglecting loops of virtual fermions, $N_{f}=0$. For an $n$-gluon scattering, $q$ equals $n-2$. Expanding Eq. (6.68) in $\epsilon$, we find

$$
\left|\mathcal{M}^{(1)}\right\rangle=-\frac{11}{3 \epsilon} C_{A}\left|\mathcal{M}^{(0)}\right\rangle+\sum_{i} \sum_{j \neq i}\left[\frac{1}{\epsilon^{2}}+\frac{1}{\epsilon} L_{i j}\right] \boldsymbol{T}_{i} \cdot \boldsymbol{T}_{j}\left|\mathcal{M}^{(0)}\right\rangle
$$


where $L_{i j}=\ln \left[\mu^{2} /\left(-s_{i j}+i 0\right)\right]$ and we suppressed helicity and momentum labels of the amplitudes. We can use

$$
\frac{11}{3} C_{A}=\frac{11}{3} \boldsymbol{T}_{g}^{2}=-\sum_{i}^{n} \sum_{j \neq i} \frac{11}{3 n} \boldsymbol{T}_{i} \cdot \boldsymbol{T}_{j}
$$

to cast Eq. (6.87) into the following form

$$
\left|\mathcal{M}^{(1)}\right\rangle=\sum_{i} \sum_{j \neq i}\left[\frac{1}{\epsilon^{2}}+\frac{1}{\epsilon}\left(\frac{11}{3 n}+L_{i j}\right)\right] \boldsymbol{T}_{i} \cdot \boldsymbol{T}_{j}\left|\mathcal{M}^{(0)}\right\rangle .
$$

If we take the matrix element of $\left|\mathcal{M}^{(1,0)}\right\rangle$ with the state of $n$-gluons with particular color indices $\left|a_{1: n}\right\rangle \equiv\left|a_{1}, \ldots a_{n}\right\rangle$ we recover the normal color-ordered amplitude, see Eq. (6.18). For example, at tree-level we find

$$
\left\langle a_{1: n} \mid \mathcal{M}^{(0)}\right\rangle=\sum_{\mathcal{P}(2, . . n-1)}\left(F^{a_{2}} \ldots F^{a_{n-1}}\right)_{a_{1} a_{n}} m_{n}\left(g_{1}, g_{2}, \ldots g_{n}\right),
$$

while the one-loop decomposition reads

$$
g_{s}^{4} c_{\Gamma}\left\langle a_{1: n} \mid \mathcal{M}^{(1)}\right\rangle=\sum_{\mathcal{P}(2, . . n) / \mathcal{R}} \operatorname{Tr}\left(F^{a_{1}} F^{a_{2}} \ldots F^{a_{n-1}} F^{a_{n}}\right) m_{n}^{(1)}\left(g_{1}, g_{2}, \ldots g_{n}\right),
$$

where $\mathcal{R}$ is the reflection transformation.

Calculating a similar matrix element in Eq. (6.89), we find

$$
\left\langle a_{1: n} \mid \mathcal{M}_{1}\right\rangle=\sum_{i} \sum_{j \neq i}\left[\frac{1}{\epsilon^{2}}+\frac{1}{\epsilon}\left(\frac{11}{3 n}+L_{i j}\right)\right]\left\langle a_{1: n}\left|\boldsymbol{T}_{i} \cdot \boldsymbol{T}_{j}\right| \mathcal{M}^{(0)}\right\rangle .
$$

We now explain how to compute the matrix elements between the state of definite color $\left|a_{1: n}\right\rangle$, the color charges $\boldsymbol{T}_{i}$ and the amplitudes $\left|\mathcal{M}^{(0)}\right\rangle$. To do so, we insert the complete set of color states

$$
\left\langle a_{1: n}\left|\boldsymbol{T}_{i} \cdot \boldsymbol{T}_{j}\right| \mathcal{M}^{(0)}\right\rangle=\sum_{b}\left\langle a_{1: n}\left|\boldsymbol{T}_{i} \cdot \boldsymbol{T}_{j}\right| b_{1: n}\right\rangle\left\langle b_{1: n} \mid \mathcal{M}^{(0)}\right\rangle,
$$

and use

$$
\left\langle a_{1: n}\left|\boldsymbol{T}_{i} \cdot \boldsymbol{T}_{j}\right| b_{1}, . ., b_{n}\right\rangle=\delta_{a_{1} b_{1}} . . i f_{a_{i} c b_{i}} \ldots i f_{a_{j} c b_{j}} . . \delta_{a_{n} b_{n}},
$$

which is true in our case since both partons $i$ and $j$ are gluons. Because $f_{a c b} \equiv$ $i F_{c b}^{a} / \sqrt{2}$, we write

$$
\begin{aligned}
& \left\langle a_{1: n}\left|\boldsymbol{T}_{i} \cdot \boldsymbol{T}_{j}\right| \mathcal{M}^{(0)}\right\rangle=-\frac{1}{2} \sum_{\mathcal{P}\left[\theta_{i j}\right]}\left(F^{a_{1}} . . F^{a_{n}}\right)_{b_{i} b_{j}} F_{c b_{i}}^{a_{i}} F_{b_{j} c}^{a_{j}} m_{n}\left(g_{i}, g_{1}, . ., g_{n}, g_{j}\right), \\
& =-\frac{1}{2} \sum_{\mathcal{P}\left[\theta_{i j}\right]} \operatorname{Tr}\left(F^{a_{i}} F^{a_{1}} . . F^{a_{n}} F^{a_{j}}\right) m_{n}\left(g_{i}, g_{1}, . ., g_{n}, g_{j}\right),
\end{aligned}
$$


where $\theta_{i j}=(1, . . i-1, i+1, ., j-1, j+1, . . n)$. Inserting this equation back into Eq. (6.92), we find

$$
\begin{aligned}
\left\langle a_{1: n} \mid \mathcal{M}^{(1)}\right\rangle= & -\frac{1}{2} \sum_{i} \sum_{j \neq i}\left[\frac{1}{\epsilon^{2}}+\frac{1}{\epsilon}\left(\frac{11}{3 n}+L_{i j}\right)\right] \\
& \times \sum_{\mathcal{P}\left[\theta_{i j}\right]} \operatorname{Tr}\left(F^{a_{i}} F^{a_{1}} . . F^{a_{n}} F^{a_{j}}\right) m_{n}\left(g_{i}, g_{1}, \ldots g_{n}, g_{j}\right) .
\end{aligned}
$$

To read off the divergences of the primitive amplitudes from Eq. (6.96), we need to identify color structures in the right hand side of that equation with color structures shown in Eq. (6.91). This can be achieved if we re-arrange the summation order in Eq. (6.96). Upon reflection, it is easy to realize that the summation over $i$ and $j$ in Eq. (6.96) reduces to the summation over indices of adjacent particles (cf. positions of $i$ and $j$ in the primitive amplitudes $m_{n}$ in Eq. (6.96)), while the summation over all permutations in Eq. (6.96) gives, upon using the reflection identities, twice the sum over permutations in Eq. (6.91). As the result, we arrive at an extremely simple formula for divergences of $n$-gluon primitive amplitudes

$$
m_{n}^{(1)}\left(g_{1}, g_{2}, \ldots g_{n}\right)=-\sum_{i=1}^{n}\left[\frac{1}{\epsilon^{2}}+\frac{1}{\epsilon}\left(\frac{11}{3 n}+L_{i, i+1}\right)\right] m_{n}^{(0)}\left(g_{1}, g_{2}, \ldots g_{n}\right),
$$

where $L_{n, n+1}=L_{n, 1}$.

\section{Colorless amplitudes}

\subsection{Colorless loop integrand in D-dimensions}

We are now in a position to discuss the color-stripped cyclic-ordered $N$ particle one-loop scattering amplitudes. In general, these amplitudes are not primitive amplitudes, although in the case of gluons color-ordered and primitive amplitudes coincide.

Since permutations of external particles are not allowed, ${ }^{9}$ when a primitive amplitude is constructed, we know all the propagators that can appear in such an amplitude. We imagine that we start from a parent diagram, so that all propagators are uniquely defined. We then find all the diagrams that contribute to the primitive amplitude by pinching propagators in the parent diagram. Hence, we write

$$
\mathcal{A}_{(\mathcal{D})}\left(\left\{p_{i}\right\},\left\{J_{i}\right\}\right)=\int \frac{d^{D} l}{i \pi^{D / 2}} \frac{\mathcal{N}\left(\left\{p_{i}\right\},\left\{J_{i}\right\} ; l\right)}{d_{0} d_{1} \cdots d_{N-1}},
$$

where $\left\{p_{i}\right\}$ and $\left\{J_{i}\right\}$ are the two sets that represent momenta and sources (polarization vectors, spinors, etc.) of the external particles. The numerator structure

\footnotetext{
${ }^{9}$ We discuss here the case where all particles have non-zero color charges.
} 
$\mathcal{N}\left(\left\{p_{i}\right\},\left\{J_{i}\right\} ; l\right)$ depends on the particle content of the theory. The denominator is a product of inverse propagators

$$
d_{i}=d_{i}(l)=\left(l+q_{i}\right)^{2}-m_{i}^{2},
$$

where the four-vector $q_{0}=0$, by convention.

In principle, the one-loop amplitude is just a collection of Feynman diagrams; therefore, it can be investigated by means of the OPP method, discussed in the previous Section. However, we point out that a key element of the OPP method is the observation that, to calculate $\mathcal{A}_{(\mathcal{D})}$, we need to know $\mathcal{N}\left(\left\{p_{i}\right\},\left\{J_{i}\right\} ; l\right)$ for values of the loop momentum $l$ where some subset of inverse propagators $\left(d_{0}, \ldots, d_{N-1}\right)$ vanishes. When an inverse propagator vanishes, the corresponding particle goes on the mass shell and the flow of the loop momentum terminates. In fact, if we put one propagator on the mass shell, the one-loop integrand becomes just a tree amplitude; if we put two propagators on the mass shell, the integrand becomes a product of two tree amplitudes, etc. Hence, we see right away that the OPP procedure is related to unitarity.

However, we need to do calculations in $D$-dimensions to regularize the ultraviolet and infrared divergences. The simplest option [23], explained in Sect. 5, is to extend the OPP procedure so that the unitarity cuts are performed in $D$-dimensions. This implies two things. First, as we already discussed in the context of the OPP reduction in the previous Section, the cut momentum is not four-dimensional and the parameterization of the residues requires introducing tensors that contain the $(D-4)$-dimensional components of the loop momentum. Second, the Lorentz indices of the internal particles must also be treated in $D$-dimensions. Ultimately, this second feature is related to the fact that not only loop momenta but also polarization vectors of various particles must be continued to higher-dimensional spacetimes, for a consistent regularization procedure. As a result, the number of spin eigenstates changes and becomes $D$-dependent.

We will be concerned with the cases where there is massless gauge boson or (massless or massive) quark in the loop. Then, a massless spin-one particle in $D_{s}$ dimensions has $D_{s}-2$ spin eigenstates while Dirac spinor in $D_{s}$ dimensions has $2^{\left(D_{s}-2\right) / 2}$ spin eigenstates. In the latter case, $D_{s}$ should be even. The spin density matrix for a massless spin-one particle with momentum $l$ and polarization vectors $e_{\mu}^{(i)}$ is given by

$$
\sum_{i=1}^{D_{s}-2} e_{\mu}^{(i)}(l) e_{\nu}^{(i)}(l)=-g_{\mu \nu}^{\left(D_{s}\right)}+\frac{l_{\mu} b_{\nu}+b_{\mu} l_{\nu}}{l \cdot b}
$$

where $b_{\mu}$ is an auxiliary light-cone vector required to fix the gauge. The spin density matrix for a fermion with momentum $l$ and mass $m$ is given by

$$
\sum_{i=1}^{2^{\left(D_{s}-2\right) / 2}} u^{(i)}(l) \bar{u}^{(i)}(l)=\sum_{\mu=1}^{D} l_{\mu} \Gamma^{\mu}+m,
$$


where $\Gamma_{\mu}$ is the $D$-dimensional generalization of the Dirac matrix.

In the preceding discussion, we introduced a special notation for the dimensionality of the internal spin space $D_{s}$, to distinguish it from the dimensionality of the space-time $D$ where the loop momentum lives. For consistency, we must choose $D \leq D_{s}$ [75]. We now generalize the notion of dimensional dependence of the one-loop scattering amplitude in Eq. (7.1) by extending the sources of all unobserved particles to a $D_{s}$-dimensional space-time

$$
\mathcal{A}_{\left(D, D_{s}\right)}\left(\left\{p_{i}\right\},\left\{J_{i}\right\}\right)=\int \frac{d^{D} l}{i \pi^{D / 2}} \frac{\mathcal{N}^{\left(D_{s}\right)}\left(\left\{p_{i}\right\},\left\{J_{i}\right\} ; l\right)}{d_{0} d_{1} \cdots d_{N-1}} .
$$

The numerator function $\mathcal{N}^{\left(D_{s}\right)}\left(\left\{p_{i}\right\},\left\{J_{i}\right\} ; l\right)$ depends explicitly on $D_{s}$ through the number of spin eigenstates of virtual particles.

A simple, but important observation is that the numerator function $\mathcal{N}^{\left(D_{s}\right)}$ (without closed fermion loops) depends linearly on $D_{s}$ provided that the external momenta and polarization vectors are four-dimensional. To generate an explicit dependence on $D_{s}$ we need to have a closed loop of contracted Lorentz-vector indices coming from vertices and propagators. At one loop we can generate at most one trace of the metric tensor. Therefore, the numerator functions of the integrand of one-loop amplitudes without closed fermion loops can always be parametrized as

$$
\mathcal{N}^{\left(D_{s}\right)}(l)=\mathcal{N}_{0}(l)+\left(D_{s}-4\right) \mathcal{N}_{1}(l) .
$$

We emphasize that there is no explicit dependence on either $D_{s}$ or $D$ in the functions $\mathcal{N}_{0,1}$. In the case of amplitudes with one closed fermion loop an additional $D_{s}$ dependence comes from the normalization of the trace of the $D_{s}$-dimensional Dirac matrices

$$
\operatorname{Tr}\left(\Gamma^{\mu} \Gamma^{\nu}\right)=t_{D_{s}} 4 g_{D_{s}}^{\mu \nu}, \quad t_{D_{s}}=\frac{1}{4} \operatorname{Tr}(1)=2^{\left(D_{s}-4\right) / 2} .
$$

This is an overall normalization factor. In traditional calculations with Feynmandiagrams its expansion in $\epsilon$ can be postponed until after the cancellation of the singularities has been carried out. Then the $\epsilon$-dependent part in Eq. (7.7) becomes irrelevant. In the $D$-dimensional unitarity cut method this additional $D_{s}$ dependence has to be taken into account and we obtain the following result for the fermion loops

$$
\mathcal{N}_{f}^{\left(D_{s}\right)}(l)=t_{D_{s}}\left(\mathcal{N}_{0, f}(l)+\left(D_{s}-4\right) \mathcal{N}_{1, f}(l)\right),
$$

At one loop we can have either a closed loop of Lorentz tensors or a closed loop of Dirac matrices. Mixed cases can appear only in the case of two or more loops. We conclude that $\mathcal{N}_{1, f}(l)=0$ and that the numerator function for closed fermion loops can be calculated in four dimensions. The $D_{s}$ dependence in that case is entirely due to the trace of the identity matrix.

For amplitudes without closed fermion loops, in a numerical implementation we need to separate the two functions $\mathcal{N}_{0,1}$. To do so, we compute the left hand 
side of Eq. (7.6) for $D_{s}=D_{1}$ and $D_{s}=D_{2}$ and, after taking appropriate linear combinations, we obtain

$$
\begin{aligned}
& \mathcal{N}_{0}(l)=\frac{\left(D_{2}-4\right) \mathcal{N}^{\left(D_{1}\right)}(l)-\left(D_{1}-4\right) \mathcal{N}^{\left(D_{2}\right)}(l)}{D_{2}-D_{1}}, \\
& \mathcal{N}_{1}(l)=\frac{\mathcal{N}^{\left(D_{1}\right)}(l)-\mathcal{N}^{\left(D_{2}\right)}(l)}{D_{2}-D_{1}}
\end{aligned}
$$

In the case of amplitudes with only bosons in the loop the numerator functions are well defined numerically for any integers $D_{1,2}>4$. If fermions are also present in the loop, then we have to choose even values for $D_{1,2}$, subject to the constraints $D_{1,2} \geq 4$.

Having established the $D_{s}$-dependence of the amplitude, we discuss the analytic continuation for sources of unobserved particles. We can interpolate $D_{s}$ either to $D_{s} \rightarrow 4-2 \epsilon$ or to $D_{s} \rightarrow 4$. The first case is known as the 't HooftVeltman (HV) scheme [45], the second case - as the four-dimensional helicity (FDH) scheme [75]. The latter scheme is of particular interest in supersymmetric (SUSY) calculations since all SUSY Ward identities are preserved. We see from Eq. (7.6) that the difference between the two schemes is simply $-2 \epsilon \mathcal{N}_{1}$.

We now substitute Eq. (7.9) into Eq. (7.5) and obtain explicit expressions for one-loop amplitudes in the HV and the FDH schemes. We derive

$$
\begin{aligned}
\mathcal{A}^{\mathrm{FDH}} & =\left(\frac{D_{2}-4}{D_{2}-D_{1}}\right) \mathcal{A}_{\left(D, D_{s}=D_{1}\right)}-\left(\frac{D_{1}-4}{D_{2}-D_{1}}\right) \mathcal{A}_{\left(D, D_{s}=D_{2}\right)}, \\
\mathcal{A}^{\mathrm{HV}} & =\mathcal{A}^{\mathrm{FDH}}-\left(\frac{2 \epsilon}{D_{2}-D_{1}}\right)\left(\mathcal{A}_{\left(D, D_{s}=D_{1}\right)}-\mathcal{A}_{\left(D, D_{s}=D_{2}\right)}\right) .
\end{aligned}
$$

We emphasize that $D_{s}=D_{1,2}$ amplitudes on the r.h.s. of Eq. (7.10) are conventional one-loop scattering amplitudes whose numerator functions are computed in higher-dimensional space-time, i.e. all internal metric tensors and Dirac gamma matrices are in integer $D_{s}=D_{1,2}$ dimensions. The loop momentum is in $D \leq D_{s}$ dimensions. It is important that no explicit dependence on the regularization parameter $\epsilon=(4-D) / 2$ is generated by spin density matrices in these amplitudes. For this reason, Eq. (7.10) allows numerical implementation. In particular, calculation of unitarity cuts in $D_{s}$ dimensions is now straightforward, as cut internal lines possess well-defined spin density matrices. Below we discuss the construction of the spin states in $D$-dimensional space-time.

\subsection{Polarization states in D-dimensions}

We begin by reminding the reader about the four-dimensional case. In four dimensions, gluons and massless quarks have two polarization states. One can use helicity states of fermions to find a useful representation of the gluon or photon polarization vectors that leads to significant simplifications of the analytic computations. Such methods are reviewed in e.g. Ref. [83] and in Appendix Appendix D. 
However, as we have seen in the previous Section, $D$-dimensional unitarity requires an analytic continuation of spin degrees of freedom to higher-dimensional space-times. In the spinor-helicity formalism, polarizations of particles of integer spin are expressed in terms of spinor solutions. It is not trivial to do this in higher-dimensional space-times, although some work in this direction has been done recently $[99,100]$. We will not discuss these issues in this Section, focusing instead on the construction of polarization states for both quarks and gluons for which the continuation to higher-dimensional spaces is straightforward.

In $D=4$ the two polarization vectors for massless gauge bosons with momentum $p$ satisfy the constraint $p \cdot \epsilon_{\lambda}=0(\lambda= \pm 1)$. We consider a general parametrization of the massless momentum $p=E(1, \sin \theta \cos \phi, \sin \theta \sin \phi, \cos \theta)$, and find the two polarization vectors

$$
\epsilon_{\lambda}(p)=\frac{1}{\sqrt{2}}\left(0, c_{\theta} c_{\phi}-\operatorname{sgn}(E) \lambda i s_{\phi}, c_{\theta} s_{\phi}+\operatorname{sgn}(E) \lambda i c_{\phi},-s_{\theta}\right),
$$

where $c_{\theta}=\cos \theta, s_{\theta}=\sin \theta, c_{\phi}=\cos \phi$ and $s_{\phi}=\sin \phi$.

To describe the explicit solution of the massless Dirac equation $\not p u=0$, we need to fix the representation of the Dirac matrices. For massless fermions, it is convenient to choose the Weyl representation where the four-dimensional $\gamma$-matrices are given by

$$
\gamma^{0}=\left(\begin{array}{ll}
\mathbf{0} & \mathbf{1} \\
\mathbf{1} & \mathbf{0}
\end{array}\right), \gamma^{i}=\left(\begin{array}{cc}
\mathbf{0} & -\sigma^{i} \\
\sigma^{i} & \mathbf{0}
\end{array}\right), \gamma^{5}=\left(\begin{array}{cc}
\mathbf{1} & \mathbf{0} \\
\mathbf{0} & -\mathbf{1}
\end{array}\right) .
$$

In this equation the boldface symbols indicate $2 \times 2$ submatrices and $\sigma^{i}$ are the Pauli matrices. Consider a massless fermion with momentum $p=\left(E, p_{x}, p_{y}, p_{z}\right)$ and let $p_{+}=E+p_{z}$. Solving the Dirac equation for massless quarks, we find

$$
u_{\lambda=1}(p)=\left(\begin{array}{c}
\sqrt{p_{+}} \\
\left(p_{x}+i p_{y}\right) / \sqrt{p_{+}} \\
0 \\
0
\end{array}\right), u_{\lambda=-1}(p)=\left(\begin{array}{c}
0 \\
0 \\
\left(p_{x}-i p_{y}\right) / \sqrt{p_{+}} \\
-\sqrt{p_{+}}
\end{array}\right)
$$

where $\lambda= \pm 1$ refers to the helicity of an incoming fermion. One can easily check that if $u(p)$ satisfies the massless Dirac equation then

$$
\frac{\vec{p} \cdot \vec{\Sigma}}{|\vec{p}|} u_{\lambda}(p)=\frac{1}{2} \gamma_{5} u_{\lambda}(p)=\frac{1}{2} \lambda u_{\lambda}(p) \text { where } \vec{\Sigma}^{i}=\frac{i}{4} \epsilon^{i j k} \gamma^{j} \gamma^{k}
$$

For anti-fermions the sign of the helicity is flipped so that $v_{\lambda}(p)=u_{-\lambda}(p)$. Because $p_{+}$vanishes for $E=-p_{z}$, the solution for the fermion moving in the $-z$ direction requires care. Taking the limit, we arrive at

$$
u_{\lambda=1}(p)=\left(\begin{array}{c}
0 \\
\sqrt{2 E} \\
0 \\
0
\end{array}\right), \quad u_{\lambda=-1}(p)=\left(\begin{array}{c}
0 \\
0 \\
\sqrt{2 E} \\
0
\end{array}\right)
$$


We are now in position to generalize the description of the polarization states to the $D$-dimensional case. We begin with the discussion of a massless gauge boson. From previous considerations we know that we must consider two dimensionalities of the loop momentum vector space, $D=4,5$ and two dimensionalities of the space where spins are embedded $D_{s} \geq D$. For example, if no quarks are present in the loop, we may take $D=4,5$ and $D_{s}=5,6$. In practice, we always compute the cut-constructible coefficients, which require only four-dimensional information, using $D=4, D_{s}=4$ to minimize the amount of algebra.

We first take $D_{s}=5$ and denote the polarization vector by $\epsilon=\left(\epsilon_{4}, \epsilon_{5}\right)$, where $\epsilon_{4}$ are the four components of the polarization vector that are embedded in the four-dimensional space. Then, if the momentum of a gluon is fourdimensional, $p=\left(\mathbf{p}_{4}, 0\right)$, there are three obvious choices for the polarization vectors: $\epsilon_{ \pm}=\left(\boldsymbol{\epsilon}_{4}^{ \pm}, 0\right)$ and $\epsilon_{0}=\left(\mathbf{0}_{4}, 1\right)$. However, if the momentum is fivedimensional $p=\left(\mathbf{p}_{4}, p_{5}\right)$, the situation is different. Note that, from a fourdimensional point of view, the massless boson with five-dimensional momentum corresponds to a massive boson in four dimensions, with the mass $p_{5}^{2}$. Hence, we can choose four-dimensional polarization vectors of a massive gauge boson, to describe the required polarization states. Explicitly, if we write the fivedimensional momentum as

$$
p=\left(E, p_{4} \sin \theta \cos \phi, p_{4} \sin \theta \sin \phi, p_{4} \cos \theta, p_{5}\right), \quad E^{2}-p_{4}^{2}=p_{5}^{2}, \quad E>0,
$$

a convenient choice of the polarization vectors is

$$
\begin{aligned}
& \epsilon^{ \pm}=\frac{1}{\sqrt{2}}(0, \cos \theta \cos \phi \mp i \sin \phi, \cos \theta \sin \phi \pm i \cos \phi,-\sin \theta, 0), \\
& \epsilon^{0}=p_{5}^{-1}\left(p_{4}, E \sin \theta \cos \phi, E \sin \theta \sin \phi, E \cos \theta, 0\right) .
\end{aligned}
$$

It is straightforward to discuss the $D_{s}=6$ case. Indeed, since the gluon momentum does not have a six-dimensional component and since we need only one additional polarization vector for $D_{s}=6$, compared to $D_{s}=5$, we can choose this additional vector to be $\epsilon_{L}=\left(\mathbf{0}_{5}, 1\right)$ since such a choice clearly satisfies the transversality constraint. This construction generalizes to higher-dimensional space-times, provided that the dimensionality of the loop momentum vector is restricted to five or less.

We now turn to the discussion of fermions. In $D_{s}$-dimensions fermions have $2^{D_{s} / 2-1}$ polarization states, given by $2^{D_{s} / 2}$-component Dirac spinors $u_{j}^{(s)}(p)$, $s=1, \ldots, 2^{D_{s} / 2-1}, j=0, \ldots, 2^{D_{S} / 2}-1$. These spinors are solutions of the massive or massless Dirac equation in $D_{s}$ dimensions

$$
\sum_{\mu=0}^{D-1}\left[p_{\mu} \Gamma_{\left(D_{s}\right)}^{\mu}-m\right] u^{(s)}(p)=0, \quad \mu=0, \ldots, D-1<D_{s},
$$

where $m$ denotes the mass of the fermion. We note that in Eq. (7.17) we treat the loop momentum as a $D$-dimensional vector, $D \leq D_{s}$, in accord with our previous discussion. 
To find solutions to Eq. (7.17), we need to explicitly construct Dirac matrices in higher dimensions. ${ }^{10}$ To this end, we consider even space-time dimensionalities and follow the recursive definition given in Ref. [101]. The Dirac matrices have to satisfy the anti-commutation relation

$$
\Gamma_{(D)}^{\mu} \Gamma_{(D)}^{\nu}+\Gamma_{(D)}^{\nu} \Gamma_{(D)}^{\mu}=2 g^{\mu \nu}, \quad \mu, \nu=0, \ldots, D-1 .
$$

We assume that the Dirac matrices for $D=D_{0}$ are known and we need to construct Dirac matrices for $D=D_{0}+2$. When we move from $D=D_{0}$ to $D=D_{0}+2$, the dimensionality of the matrices increases by a factor of two. Eq. (7.18) can be satisfied by choosing the first $D_{0}$ of the $D_{0}+2$ matrices to be given by $2^{\left(D_{0}+2\right) / 2} \times 2^{\left(D_{0}+2\right) / 2}$ block-diagonal matrices defined as follows

$$
\Gamma_{\left(D_{0}+2\right)}^{0}=\left(\begin{array}{cc}
\Gamma_{\left(D_{0}\right)}^{0} & 0 \\
0 & \Gamma_{\left(D_{0}\right)}^{0}
\end{array}\right), \quad \Gamma_{\left(D_{0}+2\right)}^{i=1, \ldots, D_{0}-1}=\left(\begin{array}{cc}
\Gamma_{\left(D_{0}\right)}^{i} & 0 \\
0 & \Gamma_{\left(D_{0}\right)}^{i}
\end{array}\right) .
$$

The remaining two matrices need to anticommute with the matrices defined in Eq. (7.19). They can be obtained by constructing an analog of the fourdimensional $\gamma_{5}$-matrix in $D_{0}$-dimensions; this is achieved by taking the product

$$
\hat{\Gamma}_{\left(D_{0}\right)}=i^{D_{0} / 2-1} \Gamma_{\left(D_{0}\right)}^{0} \Gamma_{\left(D_{0}\right)}^{1} \cdots \Gamma_{\left(D_{0}\right)}^{D_{0}-1} .
$$

The matrix $\hat{\Gamma}_{\left(D_{0}\right)}$ satisfies

$$
\hat{\Gamma}_{\left(D_{0}\right)} \Gamma_{\left(D_{0}\right)}^{\mu}+\Gamma_{\left(D_{0}\right)}^{\mu} \hat{\Gamma}_{\left(D_{0}\right)}=0, \quad \mu=0, \ldots, D_{0}-1 .
$$

Using $\hat{\Gamma}_{\left(D_{0}\right)}$, we can construct the two missing matrices, to complete the algebra of Dirac matrices in $D_{0}+2$ dimensions

$$
\Gamma_{\left(D_{0}+2\right)}^{D_{0}}=\left(\begin{array}{cc}
0 & \hat{\Gamma}_{\left(D_{0}\right)} \\
-\hat{\Gamma}_{\left(D_{0}\right)} & 0
\end{array}\right), \quad \Gamma_{\left(D_{0}+2\right)}^{D_{0}+1}=\left(\begin{array}{cc}
0 & i \hat{\Gamma}_{\left(D_{0}\right)} \\
i \hat{\Gamma}_{\left(D_{0}\right)} & 0
\end{array}\right) .
$$

The recursive construction requires an initial condition. In this case, the appropriate condition is clear since it is given by the familiar algebra of Dirac matrices in four-dimensional space-time. We note that the above construction is independent of the four-dimensional representation of the Dirac matrices, except for the initial condition. This is a welcome feature since it allows us to use the same formalism for both Weyl and Dirac representations. In the following we suppress the dimensionality index $D$, so that $\Gamma^{\mu}$ denotes a $D$-dimensional gamma-matrix. We also note that within this framework, the four-dimensional $\gamma_{5}$ matrix is continued to $D$-dimensions following the prescription by 't Hooft and Veltman [45]. Indeed, they defined the continuation of $\gamma_{5}$ as a matrix that commutes with $\gamma_{\mu}$ if $\mu>3$ and anticommutes otherwise. It is easy to see that

\footnotetext{
${ }^{10}$ With a slight abuse of notation, we shall talk about $D$-dimensional Dirac algebra, rather than $D_{s}$-dimensional Dirac algebra throughout the text until Eq. (7.30).
} 
the appropriate continuation of $\gamma_{5}$ to $D_{0}+2$ dimensions is given by a blockdiagonal matrix with four-dimensional $\gamma_{5}$ along the diagonal. As an example, in six dimensions we have

$$
\Gamma^{\gamma_{5}}=\left(\begin{array}{cc}
\gamma_{5} & \mathbf{0} \\
\mathbf{0} & \gamma_{5}
\end{array}\right)
$$

The recursive structure of the $D$-dimensional gamma-matrices allows us to solve the Dirac equation recursively. We first discuss massless solutions. Consider a massless fermion with momentum $p$, in $D$-dimensions. To construct explicit polarization states for such a fermion, we employ an auxiliary light-like vector $n$ such that $n \cdot p \neq 0$. We write

$$
u^{(s)}(p, n)=\frac{\hat{p}}{\sqrt{2 p \cdot n}} \chi_{s}^{(D)}(n), \quad \bar{u}^{(s)}(p, n)=\bar{\chi}_{s}^{(D)}(n) \frac{\hat{p}}{\sqrt{2 p \cdot n}} .
$$

Here $\hat{p}=p_{\mu} \Gamma^{\mu}$, where the index $\mu$ runs over $0,1, \ldots D-1$ components. The index $s$ specifies the spinor polarization states. Note that when the Dirac conjugate spinor is constructed, the spinor momentum $p$ is not complex conjugated. This is an irrelevant detail if the on-shell momentum is real, but it becomes important for consistent applications within generalized unitarity where calculations with on-shell complex momenta are required.

We choose $D$-dimensional, $p$-independent spinors $\chi_{s}^{(D)}(n)$ in such a way that their direct product reads

$$
\sum_{s=1}^{2^{(D / 2-1)}} \chi_{s}^{(D)}(n) \otimes \bar{\chi}_{s}^{(D)}(n)=\hat{n} .
$$

Then, it is easy to see that the $u_{j}(p, n)$ spinors satisfy both the Dirac equation for massless fermions and the completeness relation

$$
\sum_{s=1}^{2^{(D / 2-1)}} u^{(s)}(p, n) \otimes \bar{u}^{(s)}(p, n)=\frac{\hat{p} \hat{n} \hat{p}}{2 p \cdot n}=\hat{p} .
$$

We conclude that $u^{(s)}(p, n)$ is a valid choice for the on-shell fermion wave functions.

The above construction involves an auxiliary vector $n$ and, for this reason it is quite flexible. Having such a flexibility turns out to be important, especially since we have to construct on-shell spinors for complex momenta. We give a few examples below. We consider a $D$-dimensional vector $n=$ $\left(n_{0}, n_{x}, n_{y}, n_{z},\left\{n_{i \in(D-4)}\right\}\right)$, choose $n_{0}=1 / 2, n_{z}=1 / 2$ and set all other components to zero. We need to find the spinors $\chi$ such that

$$
\sum_{s=1}^{2^{(D / 2-1)}} \chi_{s}^{(D)}(n) \otimes \bar{\chi}_{s}^{(D)}(n)=\hat{n}=\frac{1}{2}\left(\Gamma^{0}-\Gamma^{z}\right) .
$$

Since $\Gamma^{0, x, y, z}$ are all block-diagonal [101], with "blocks" being $4 \times 4$ matrices, a $D$-dimensional spinor is constructed by simple iteration of the four-dimensional 
spinor. We assume that the four-dimensional Weyl representation is extended to $D$-dimensions. The four-dimensional spinors are given by

$$
\chi_{1}^{(4)}=\left(\begin{array}{l}
1 \\
0 \\
0 \\
0
\end{array}\right), \quad \chi_{2}^{(4)}=\left(\begin{array}{c}
0 \\
0 \\
0 \\
-1
\end{array}\right) .
$$

In six dimensions the eight-component spinors are chosen to be

$$
\chi_{1,2}^{(6)}=\left(\begin{array}{c}
\chi_{1,2}^{(4)} \\
0
\end{array}\right), \chi_{3,4}^{(6)}=\left(\begin{array}{c}
0 \\
\chi_{1,2}^{(4)}
\end{array}\right) .
$$

The case $D=8$ is obtained again by iteration. We find

$$
\begin{aligned}
& \chi_{1,2}^{(8)}=\left(\begin{array}{c}
\chi_{1,2}^{(6)} \\
0
\end{array}\right), \quad \chi_{3,4}^{(8)}=\left(\begin{array}{c}
\chi_{3,4}^{(6)} \\
0
\end{array}\right), \\
& \chi_{5,6}^{(8)}=\left(\begin{array}{c}
0 \\
\chi_{1,2}^{(6)}
\end{array}\right), \quad \chi_{7,8}^{(8)}=\left(\begin{array}{c}
0 \\
\chi_{3,4}^{(6)}
\end{array}\right) .
\end{aligned}
$$

We now present two alternative procedures to define fermionic spinors which we employ when a particular choice of the vector $n$ leads to numerical instabilities. This occurs for the on-shell momentum $p=\left(p_{0}, 0,0, p_{0}\right)$ since $(p \cdot n)=0$. To handle this case, we change the vector $n$ to $\left(1 / 2,0,0,-1 / 2,0_{D-4}\right)$ in the above formulas. However, even this can be insufficient. Indeed, note that a complex momentum $p=\left(0, p_{x}, p_{y}, 0\right)$ can be light-like and, therefore, be a valid on-shell momentum for a massless fermion. Clearly $p \cdot n=0$ in this case, for the two choices of the vector $n$ that we discussed. To deal with this case, we need to choose yet another $n$. We can take $n=\left(1,1,0,0,0_{D-4}\right)$ and choose the following four-dimensional spinors

$$
\chi_{1}^{(4)}=\left(\begin{array}{l}
1 \\
0 \\
0 \\
1
\end{array}\right), \quad \chi_{2}^{(4)}=\left(\begin{array}{l}
0 \\
1 \\
1 \\
0
\end{array}\right)
$$

The higher-dimensional spinors are obtained from these four-dimensional solutions along the lines discussed above.

This procedure can be easily extended to the case of massive fermions. We will discuss such an extension assuming that the four-dimensional gammamatrices are in the Dirac representation. We need to explicitly construct the $2^{D_{s} / 2-1}$ spin polarization states $u_{j}^{(s)}(p, m)$ that satisfy the Dirac equation Eq. (7.17) and the completeness relation

$$
\sum_{s=1}^{2^{\left(D_{s} / 2-1\right)}} u_{i}^{(s)}(p, m) \bar{u}_{j}^{(s)}(p, m)=\sum_{\mu=0}^{D-1} p_{\mu} \Gamma_{i j}^{\mu}+m \times \delta_{i j} .
$$


The on-shell condition for a fermion with the mass $m$ and momentum $p$ is taken to be $p^{2}=m^{2}$. To construct a set of $2^{D_{s} / 2-1}$ spinors satisfying the Dirac equation we generalize the procedures used in the massless case. We define the spinors

$$
u^{(s)}(p, m)=\frac{\left(p_{\mu} \Gamma^{\mu}+m\right)}{\sqrt{p_{0}+m}} \eta_{D_{s}}^{(s)}, \quad s=1, \ldots, 2^{D_{s} / 2-1} .
$$

For $D_{s}=4$ we choose

$$
\eta_{1}^{(4)}=\left(\begin{array}{l}
1 \\
0 \\
0 \\
0
\end{array}\right), \quad \eta_{2}^{(4)}=\left(\begin{array}{l}
0 \\
1 \\
0 \\
0
\end{array}\right),
$$

and construct recursively the $D_{s}=6$ eight-component basis spinors

$$
\eta_{1,2}^{(6)}=\left(\begin{array}{c}
\eta_{1,2}^{(4)} \\
0
\end{array}\right), \quad \eta_{3,4}^{(5)}=\left(\begin{array}{c}
0 \\
\eta_{1,2}^{(4)}
\end{array}\right) .
$$

The eight spinors for $D_{s}=8$ are obtained using the obvious generalization. It is easy to see that the spinors constructed in this way do indeed satisfy the Dirac equation. To check the completeness relation, we define the Dirac conjugate spinor to be

$$
\bar{u}^{(s)}(p, m)=\bar{\eta}_{D_{s}}^{(s)} \frac{\left(p_{\mu} \Gamma^{\mu}+m\right)}{\sqrt{p_{0}+m}} .
$$

Then it is easy to see that Eq. (7.31) is satisfied.

\subsection{Berends-Giele recursion relations}

Any calculation based on unitarity cuts requires on-shell scattering amplitudes for complex on-shell momenta. For $D$-dimensional generalized unitarity, these amplitudes must be computed in $D$-dimensional space-time which implies that momenta of some particles in the scattering amplitude and their polarization states are continued to $D$-dimensions. How do we obtain such scattering amplitudes? It turns out that the most robust method that allows a fast and efficient computation of the on-shell scattering amplitudes is based on the recursion relations by Berends and Giele [102]. Here we summarize the main idea of this method and give some examples.

Consider a color-ordered $(n+1)$-gluon scattering amplitude at tree level. We take one of the gluons off the mass-shell, and remove its polarization vector. The resulting object is called the gluon current ${ }^{11}$; it is denoted by $G^{\mu}\left(g_{1}, g_{2}, . ., g_{n}\right)$. The arguments of the current refer to ordered gluons with outgoing momenta and polarization vectors, i. e. $g_{i}=\left(k_{i}, \epsilon_{i}\right)$. The outgoing momentum of the offshell line is $-k_{1}-k_{2}-\ldots . k_{n}$, due to the momentum conservation. A simple and

\footnotetext{
${ }^{11}$ Note that in Ref. [102] the currents also include the gluon propagator of the off-shell gluon. We choose to define currents without that propagator, to be as close as possible to a numerical implementation of Berends-Giele recursion relations.
} 


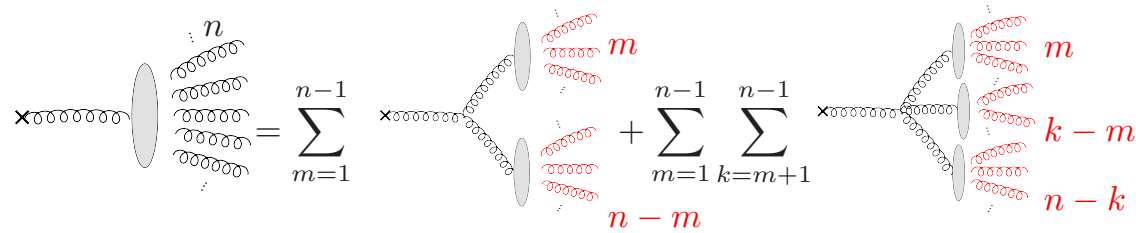

Figure 7.1: Recursion relation for the $n$-gluon current, Eq. (7.36), the integers denote the number of gluons involved in the current.

remarkably robust observation is that there exists a recursion relation that the gluon current satisfies. One can understand this equation by tracing back the "off-shell" gluon and realizing that it can split into two or three off-shell currents, thanks to the presence of three- and four-gluon vertices in QCD. Because such a splitting can not violate the ordering of the external gluons, the number of terms that contribute is limited. The recurrence relation reads

$$
\begin{aligned}
& G^{\mu}\left(g_{1}, g_{2}, \ldots g_{n}\right)=\sum_{m=1}^{n-1} V_{3}^{\mu \nu \rho}\left(-k_{1 ; n}, k_{1 ; m}, k_{m+1 ; n}\right) S_{\nu \nu^{\prime}}^{G}\left(k_{1 ; m}\right) S_{\rho \rho^{\prime}}^{G}\left(k_{m+1 ; n}\right) \\
& \quad \times G^{\nu^{\prime}}\left(g_{1}, g_{2}, \ldots, g_{m}\right) G^{\rho^{\prime}}\left(g_{m+1}, \ldots, g_{n}\right) \\
& +\sum_{m=1}^{n-1} \sum_{k=m+1}^{n-1} V_{4}^{\mu \nu \rho \sigma}\left(-k_{1 ; n}, k_{1 ; m}, k_{m+1 ; k}, k_{k+1 ; n}\right) S_{\nu \nu^{\prime}}^{G}\left(k_{1 ; m}\right) S_{\rho \rho^{\prime}}^{G}\left(k_{m+1 ; k}\right) \\
& \times S_{\sigma \sigma^{\prime}}^{G}\left(k_{k+1 ; n}\right) G^{\nu^{\prime}}\left(g_{1}, \ldots, g_{m}\right) G^{\rho^{\prime}}\left(g_{m+1}, \ldots, g_{k}\right) G^{\sigma^{\prime}}\left(g_{k+1}, \ldots, g_{n}\right) .
\end{aligned}
$$

In Eq. (7.36), $V_{3,4}$ are the three- and four-gluon color-stripped vertices, see Fig. 7.1, $S_{\mu \nu}^{G}(p)$ is the gluon propagator and $k_{i ; j}=\sum_{m=i}^{j} k_{m}$. The on-shell colorordered physical amplitude for the $(n+1)$-gluon scattering is obtained from the on-shell limit $k_{1 ; n}^{2} \rightarrow 0$ of the $n$-gluon current $G^{\mu}\left(g_{1}, g_{2}, \ldots g_{n}\right)$, contracted with the polarization vector of the gluon with momentum $k_{1 ; n}$. The BerendsGiele procedure is recursive since it expresses currents of higher multiplicities through currents of lower multiplicities. The initial condition for the recursion is $G^{\mu}\left(g_{1}\right)=\epsilon_{1}^{\mu}$, so that the one-gluon current is just the polarization vector of that gluon. It is an important feature of the Berends-Giele construction that currents can be easily computed for both, complex momenta and in space-times of higher dimensionality. This makes Berends-Giele recursion an ideal tool to use with generalized $D$-dimensional unitarity.

The Berends-Giele recursion can be easily generalized to cases with fermions, gluons and electroweak vector bosons. Recall that when fermions are involved, we compute primitive, rather than color-ordered, amplitudes using color-stripped Feynman rules, Fig. 6.1. An interesting consequence of color ordering is that the sign of the color-stripped quark-gluon vertex depends on whether, when progressing along the fermion line towards the vertex facing in the direction of 


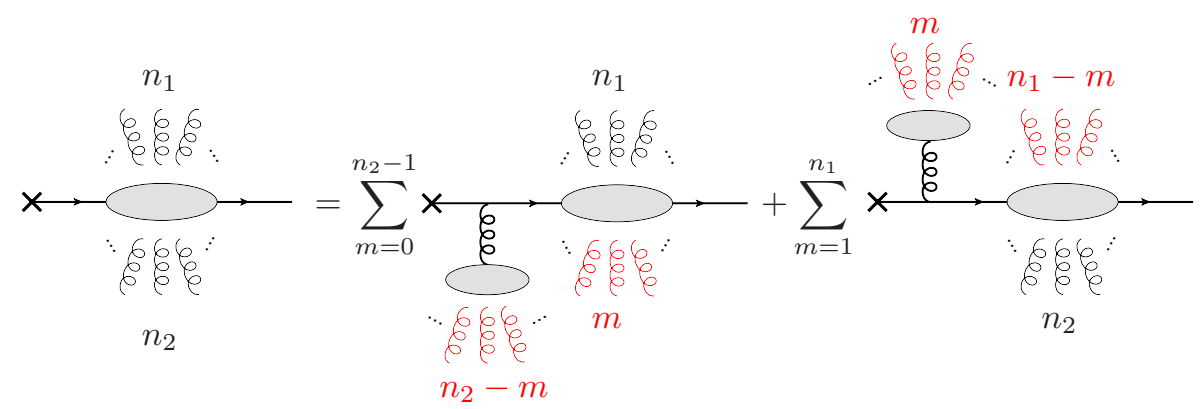

Figure 7.2: Recursion relation for the two-quark $n$-gluon current, Eq. (7.37).

the arrow, the gluon appears on the right-hand side or on the left-hand side. The simplest current that one can define in this case is the current that gives the primitive amplitude for two-quark and $n$-gluon scattering. We define the current by taking the incoming fermion line off shell; we denote the corresponding current by $\bar{Q}\left(g_{1}, g_{2}, \ldots, g_{n_{1}} ; q ; g_{n_{1}+1}, g_{n+2} \ldots g_{n_{1}+n_{2}}\right)$, with $q$ being the outgoing fermion. The recursion relation for $\bar{Q}$ reads

$$
\begin{aligned}
\bar{Q}= & \frac{i}{\sqrt{2}} \sum_{m=0}^{n_{2}-1} G^{\nu}\left(g_{n_{1}+m+1}, . ., g_{n_{1}+n_{2}}\right) \bar{Q}\left(g_{1}, . . g_{n_{1}} ; q ; g_{n_{1}+1}, . . g_{n_{1}+m}\right) \\
& \times \hat{S}^{F}\left(p_{q}+k_{1 ; n_{1}+m}\right) \hat{\Gamma}^{\mu} S_{\mu \nu}^{G}\left(k_{n_{1}+m+1 ; n_{1}+n_{2}}\right) \\
- & \frac{i}{\sqrt{2}} \sum_{m=1}^{n_{1}} G^{\nu}\left(g_{1}, . ., g_{m}\right) \bar{Q}\left(g_{m+1}, . . g_{n_{1}} ; q ; g_{n_{1}+1}, . . g_{n_{2}}\right) \\
& \times \hat{S}^{F}\left(p_{q}+k_{m+1 ; n_{1}+n_{2}}\right) \hat{\Gamma}^{\mu} S_{\mu \nu}^{G}\left(k_{1 ; m}\right),
\end{aligned}
$$

where $p_{q}$ is the momentum carried by the outgoing fermion and $\hat{S}^{F}(p)=i /(\hat{p}-$ $m)$ is a fermion propagator. We have used the notation $\hat{\Gamma}_{\mu}$ in Eq. (7.37) to denote the $D$-dimensional Dirac matrix and to emphasize that the recursion relation can be continued to higher-dimensional space-times in a straightforward way. This relation is illustrated in Fig. 7.2. The initial condition for the current $\bar{Q}$ is given by the massless or massive Dirac-conjugate spinor $\bar{u}$.

As a further example, we present the recursion relation for the current that defines the primitive amplitude that involves two quark-pairs of the same flavour and an arbitrary number of gluons. To define the current, we take one of the incoming fermions off the mass shell. All partons are ordered; the current is written as $\bar{Q}_{Q \bar{Q} Q}\left(g_{1 \ldots n_{1}} ; q_{1} ; g_{n_{1}+1, \ldots, n_{12}} ; \bar{q}_{2} ; g_{n_{12}+1, \ldots, n_{123}} ; q_{3} ; g_{n_{123}+1, \ldots, n_{1234}}\right)$. Here, $q_{1}, \bar{q}_{2}$ and $q_{3}$ are the outgoing quark, anti-quark and quark respectively, $n_{i j k \ldots}=n_{i}+n_{j}+n_{k}+\ldots$, and $g_{i_{1}+1, \ldots i_{2}}$ denote the $i_{2}-i_{1}$ gluons $g_{i_{1}+1} \ldots g_{i_{2}}$. 
The recursion relation for $\bar{Q}_{Q \bar{Q} Q}$ reads

$$
\begin{aligned}
& \bar{Q}_{Q \bar{Q} Q}\left(g_{1 \ldots n_{1}} ; q_{1} ; g_{n_{1}+1, \ldots, n_{12}} ; \bar{q}_{2} ; g_{n_{12}+1, \ldots, n_{123}} ; q_{3} ; g_{n_{123}+1, \ldots, n_{1234}}\right)= \\
& \frac{-i}{\sqrt{2}} \sum_{m=1}^{n_{1}} \bar{Q}_{Q \bar{Q} Q}\left(g_{m+1, \ldots, n_{1}} ; q_{1} ; g_{n_{1}+1, \ldots, n_{12}} ; \bar{q}_{2} ; g_{n_{12}+1, \ldots, n_{123}} ; q_{3} ; g_{n_{123}+1, \ldots, n_{1234}}\right) \\
& \quad \times \hat{S}^{F}\left(q_{123}+g_{m+1 ; n_{1234}}\right) \hat{\Gamma}^{\mu} G^{\nu}\left(g_{1 \ldots m}\right) S_{\mu \nu}^{G}\left(g_{1 ; m}\right) \\
& +\frac{i}{\sqrt{2}} \sum_{m=0}^{n_{2}} \bar{Q}\left(g_{1}, . . g_{n_{1}} ; q_{1} ; g_{n_{1}+1}, . . g_{n_{1}+m}\right) \hat{S}^{F}\left(q_{1}+g_{1 ; n_{1}+m}\right) \hat{\Gamma}^{\mu} \\
& \times S_{\mu \nu}^{G}\left(q_{23}+g_{n_{1}+m+1 ; n_{1234}}\right) G_{\bar{Q} Q}^{\nu}\left(g_{n_{1}+m+1 ; n_{12}} ; \bar{q}_{2} ; g_{n_{12}+1, \ldots, n_{123}} ; q_{3} ; g_{n_{123}+1, \ldots, n_{1234}}\right) \\
& -\frac{i}{\sqrt{2}} \sum_{m=0}^{n_{3}} \bar{Q}\left(g_{n_{12}+m+1, \ldots, n_{123}} ; q_{3} ; g_{n_{123}+1, \ldots, n_{1234}}\right) \hat{S}^{F}\left(q_{3}+g_{n_{12}+m+1, n_{1234}}\right) \hat{\Gamma}^{\mu} \\
& \times G_{Q \bar{Q}}^{\nu}\left(g_{1 \ldots n_{1}} ; q_{1} ; g_{n_{1}+1, \ldots, n_{12}} ; \bar{q}_{2} ; g_{n_{12}+1, \ldots, n_{12}+m}\right) S_{\mu \nu}^{G}\left(q_{12}+g_{1 ; n_{12}+m}\right) \\
& +\frac{i}{\sqrt{2}} \sum_{m=0}^{n_{4}-1} \bar{Q}_{Q \bar{Q} Q}\left(g_{1, \ldots, n_{1}} ; q_{1} ; g_{n_{1}+1, \ldots, n_{12}} ; \bar{q}_{2} ; g_{n_{12}+1, \ldots, n_{123}} ; q_{3} ; g_{n_{123}+1, \ldots, n_{123}+m}\right) \\
& \times \hat{S}^{F}\left(q_{123}+g_{1 ; n_{123}+m}\right) \hat{\Gamma}^{\mu} G^{\nu}\left(g_{n_{123}+m+1 \ldots n_{1234}}\right) S_{\mu \nu}^{G}\left(g_{n_{123}+m+1 ; n_{1234}}\right) .
\end{aligned}
$$

In Eq. (7.38) $G_{Q \bar{Q}}^{\mu}$ and $G_{\bar{Q} Q}^{\mu}$ denote currents with an off-shell gluon, a quarkantiquark pair and an arbitrary number of gluons in the final state. We do not give the explicit recursion relations for these currents, since they are very similar to the recursion relation shown in Eq. (7.38). Eq. (7.38) holds also for four-quark amplitudes with two distinct quark flavours as long as the gluon currents $G_{Q \bar{Q}}$ and $G_{\bar{Q} Q}$ are set to zero. It is clear that the right hand side of Eq. (7.38) involves simpler lower-multiplicity currents. This relation is illustrated in Fig. 7.3. The initial condition for the current can be obtained by setting $n_{i}=0, i=1,2,3,4$, in Eq. (7.38). One obtains

$$
\begin{aligned}
\bar{Q}_{Q \bar{Q} Q}\left(q_{1} ; \bar{q}_{2} ; q_{3}\right) & =\frac{i}{\sqrt{2}} \bar{Q}\left(q_{1}\right) \hat{S}^{F}\left(q_{1}\right) \hat{\Gamma}^{\mu} G_{\bar{Q} Q}^{\nu}\left(\bar{q}_{2} ; q_{3}\right) \hat{S}_{\mu \nu}^{G}\left(q_{23}\right) \\
& -\frac{i}{\sqrt{2}} \bar{Q}\left(q_{3}\right) \hat{S}^{F}\left(q_{3}\right) \hat{\Gamma}^{\mu} G_{Q \bar{Q}}^{\nu}\left(q_{1} ; \bar{q}_{2}\right) \hat{S}_{\mu \nu}^{G}\left(q_{12}\right) .
\end{aligned}
$$

The above construction generalizes to even more complicated currents, with e.g. larger number of fermion pairs. Currents that satisfy recurrence relations can also be constructed to compute scattering amplitudes with color-neutral particles, that are required to describe the production of the electroweak gauge bosons and the Higgs boson. Such currents have been heavily used in recent one-loop computations, see e.g. Refs. [11, 6, 10, 103, 15].

\subsection{Britto-Cachazo-Feng-Witten relations for on-shell amplitudes}

Berends-Giele recursion relations constitute an important tool for numerical computations of one-loop scattering amplitudes. There are however also a number of cases when those relations can be used to derive properties of scattering 


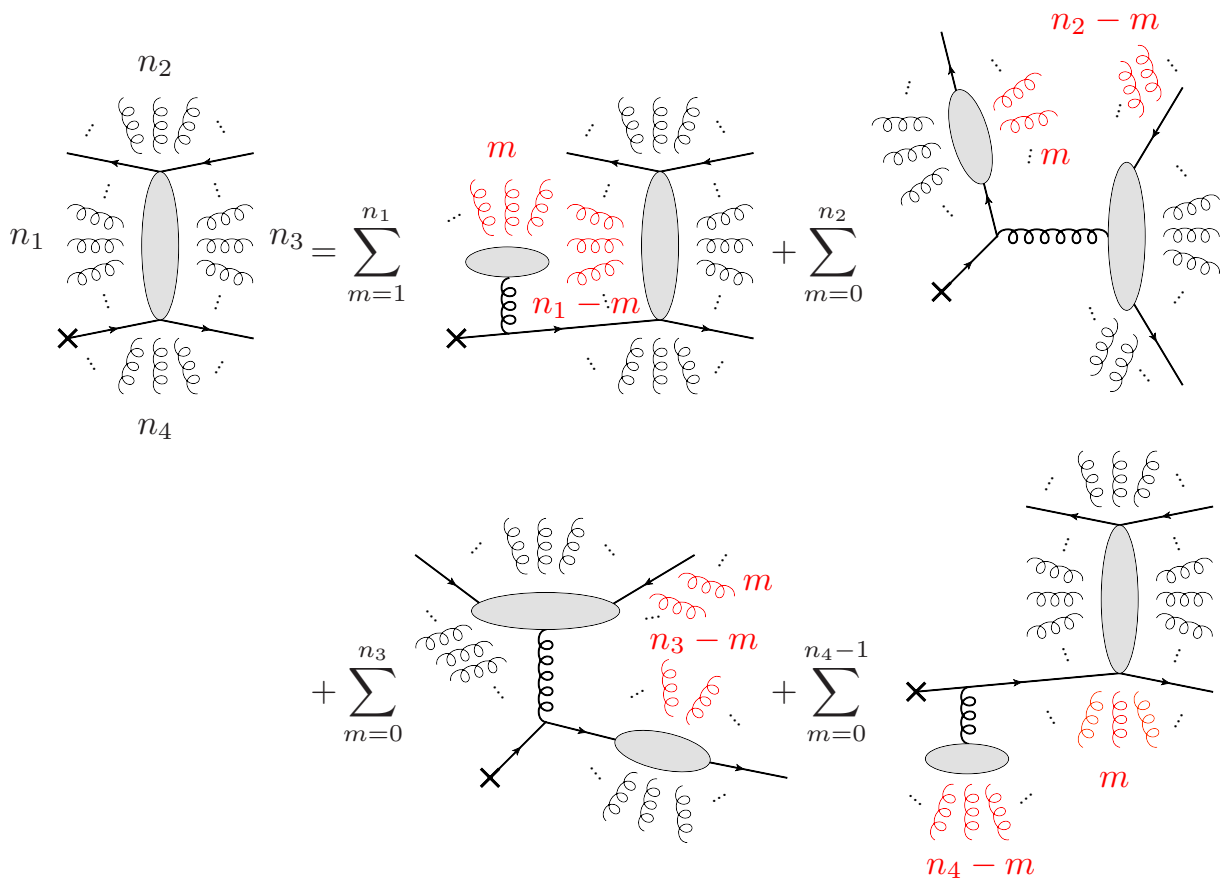

Figure 7.3: Recursion relation for the four-quarks and n-gluon current, Eq. (7.38).

amplitudes analytically. Interesting examples can be found in a review [83] and we do not repeat them here. In this Section, we describe a different example of the application of the Berends-Giele recursion - a derivation of relations between tree-level on-shell scattering amplitudes involving a different number of particles, first discovered by Britto, Cachazo, Feng and Witten (BCFW) [104].

We begin by considering the $n$-gluon color-ordered tree-level scattering amplitude $\mathcal{M}\left(g_{\lambda_{1}}, g_{\lambda_{2}}, . ., g_{\lambda_{n}}\right)$, where all gluon momenta are incoming. We assume that $n>4$. It is well-known that, in order to be non-zero, the amplitude must contain at least two helicities (say, minus one) that differ from all the other helicities (say, plus one). We fix the helicity of the first gluon to be negative; then, it is always possible to find a gluon of positive helicity in a position that is not adjacent to the gluon $g_{1}$. We will therefore study the amplitude $\mathcal{M}\left(1^{-}, \ldots, j^{+}, \ldots, n\right), j \neq 2, n$.

Following [105], it is convenient to choose a reference frame where the momentum of the gluon $g_{1}$ is $p_{1}=\mu\left(1, \mathbf{0}_{\perp}, 1\right)$ and the momentum of the gluon $g_{j}$ is $p_{j}=\mu\left(1, \mathbf{0}_{\perp},-1\right)$, where $\mu$ is a constant with the mass dimension one. We choose the polarization vectors to be $\epsilon_{1}^{+}=\epsilon_{j}^{-}=q / \sqrt{2}$, where $q=(0,1, i, 0)$. We can now deform the momenta of the gluons $g_{1, j}$ by shifting them in the $q$-direction

$$
p_{1} \rightarrow p_{1}(z)=p_{1}+z \mu q, \quad p_{j} \rightarrow p_{j}(z)=p_{j}-z \mu q,
$$


where $z$ is a complex parameter. It follows from Eq. (7.40) that $p_{1}(z)+p_{j}(z)=$ $p_{1}+p_{j}$ and, since vectors $p_{1, j}$ and $q$ are orthogonal, $p_{1}^{2}(z)=p_{j}^{2}(z)=0$. Finally, $p_{1}(z) \cdot \epsilon_{1}=0$ and $p_{j}(z) \cdot \epsilon_{j}=0$, which implies that both $\epsilon_{1}$ and $\epsilon_{j}$ are valid polarization vectors for gluons with shifted momenta.

If the amplitude $\mathcal{M}\left(g_{\lambda_{1}, z}, \ldots g_{\lambda_{j}, z}, \ldots g_{\lambda_{n}}\right)$ is calculated with the momenta $p_{1, j}(z)$, it becomes a rational function of $z$ since the only place such a dependence enters is through the momenta of gluons $g_{1}$ and $g_{j}$. If we assume that the amplitude $\mathcal{M}(z)$ vanishes at $z=\infty$, so does the integral of $\mathcal{M}(z)$ over an infinitely remote contour

$$
\oint_{|z|=\infty} \frac{\mathrm{d} z}{z} \mathcal{M}(z)=0 .
$$

The integration in Eq. (7.41) can be performed using Cauchy's theorem. We obtain

$$
\mathcal{M}(0)+\sum_{z=z_{\alpha} \neq 0} \operatorname{Res}\left[\frac{\mathcal{M}(z)}{z}\right]=0
$$

where the sum goes over all poles of the amplitude $\mathcal{M}(z)$ in the complex $z$-plane.

To understand where these poles come from, we consider all (cyclic) ordered partitions of the set $\pi=\{1,2, . . j, j+1, \ldots n\}$ into two sets $\pi=\left\{\pi_{1}^{\alpha} \cup \pi_{j}^{\alpha}\right\}$, $\alpha=1, \ldots \alpha_{\max }$ such that $\pi_{1}^{\alpha}$ contains $g_{1}$ and $\pi_{j}^{\alpha}$ contains $g_{j}$. We consider now the value of the parameter $z$ close to $z_{\alpha}$, defined by the following equation

$$
z_{\alpha}=-\frac{P_{\pi_{1}^{\alpha}}^{2}}{2 \mu P_{\pi_{1}^{\alpha}} \cdot q}, \quad P_{\pi_{1}^{\alpha}}=\sum_{i \subset \pi_{1}^{\alpha}} p_{i},
$$

and observe that the dominant contribution to the scattering amplitude comes from the resonant term

$$
\begin{aligned}
\lim _{z \rightarrow z^{\alpha}} \frac{\mathcal{M}(z)}{z} & \approx \frac{-i \mathcal{M}^{\mu}\left(\pi_{1}^{\alpha}\right) g_{\mu \nu} \mathcal{M}^{\nu}\left(\pi_{j}^{\alpha}\right)}{2 \mu\left(P_{\pi_{1}^{\alpha}} \cdot q\right)\left(z-z_{\alpha}\right) z_{\alpha}} \\
& =\frac{i}{\left(z-z_{\alpha}\right) P_{\pi_{1}^{\alpha}}^{2}} \times \sum_{\lambda= \pm} \mathcal{M}\left(\pi_{1}^{\alpha}, \lambda\right) \mathcal{M}\left(\pi_{j}^{\alpha}, \lambda\right) .
\end{aligned}
$$

In the second step in Eq. (7.44), we used gauge invariance of on-shell amplitudes to replace the metric tensor $g_{\mu \nu}$ with the sum over polarizations of the nearly on-shell, intermediate gluon line. We use Eq. (7.44) to compute the residue in Eq. (7.42) and obtain the final expression for the on-shell scattering amplitude

$$
\mathcal{M}(0)=-i \sum_{\alpha=1}^{\alpha_{\max }} \sum_{\lambda= \pm} \frac{\mathcal{M}\left(\pi_{1}^{\alpha}, \lambda\right) \mathcal{M}\left(\pi_{j}^{\alpha}, \lambda\right)}{P_{\pi_{1}^{\alpha}}^{2}}
$$

The striking feature of Eq. (7.45) is that it expresses the desired on-shell scattering amplitude through on-shell scattering amplitudes of lower multiplicities, evaluated at complex on-shell momenta. This relation between scattering amplitudes was derived in Ref. [104]; it is known as the BCFW relation. 
The derivation of the BCFW relation, that we just described, relies on the fact that the amplitude $\mathcal{M}(z)$ vanishes at $z \rightarrow \infty$. To prove this feature of QCD amplitudes, we will use Berends-Giele recursion relations. We begin by deriving constraints imposed by gauge invariance. If we write the scattering amplitude as $\mathcal{M}(z)=\epsilon_{1, \mu}(z) A^{\mu}(z)$, gauge invariance requires $p_{1}^{\mu}(z) A_{\mu}(z)=0$. Using the explicit form of $p_{1}(z)$ and the fact that $\epsilon_{1, \mu}=q_{\mu} / \sqrt{2}$, we find

$$
\epsilon_{1, \mu} A^{\mu}(z)=-\frac{p_{1}^{\mu} A_{\mu}(z)}{\sqrt{2} \mu z} .
$$

To compute $A_{\mu}(z)$, we can use Berends-Giele recursion relation for the gluon current. The recursion relation for the gluon current involves two or three lowermultiplicity currents. One of those currents includes gluon $g_{j}$ that, in the limit of large $z$, carries large momentum; we will refer to such a current as "hard" . We denote the hard (soft) current by $H_{\mu}\left(S_{\mu}\right)$ and the (outgoing) momentum that it carries by $p_{H}\left(p_{S}\right)$. In contrast to the previous Section, it is convenient to include propagators associated with off-shell legs into the definition of the currents. Contracting the hard and soft currents with three- and four-gluon vertices, and using the transversality of the currents, we obtain

$$
\begin{aligned}
& H_{\mu} \sim\left(p_{H}+p_{S}\right)^{-2}\left(2\left(p_{H} \cdot S\right) H_{\mu}+\left(p_{S}-p_{H}\right)_{\mu}(H \cdot S)\right. \\
&\left.-2\left(p_{S} \cdot H\right) S_{\mu}+V_{4, \mu}(S, H, S)\right) .
\end{aligned}
$$

The large- $z$ limit corresponds to $p_{H}=-z \mu q+\mathcal{O}(1), p_{S} \sim O(1)$. We will make the assumption that the hard current scales with $z$ as $H \sim \mathcal{O}(1)$ and we will show that the recursion relation is consistent with this scaling. To this end note that, under the scaling assumption, the four-gluon vertex contribution in Eq. (7.47) is suppressed as $z^{-1}$ and can be disregarded. We obtain

$$
\begin{aligned}
H_{\mu} & \sim z^{-1}\left(2 z(q \cdot S) H_{\mu}-z q_{\mu}(H \cdot S)-2\left(p_{s} \cdot H\right) S_{\mu}\right) \\
& \sim(2 q \cdot S) H_{\mu}-q_{\mu}(H \cdot S)+\mathcal{O}\left(z^{-1}\right),
\end{aligned}
$$

so that indeed $H_{\mu} \sim \mathcal{O}(1)$ in the large- $z$ limit. We note that at large $z$ there are two terms in the recursion relation - one that is proportional to $H_{\mu}$ and the other proportional to $q_{\mu}$. Since the initial condition for the recursion is $H_{\mu}=\epsilon_{j, \mu} \sim q_{\mu}$, we conclude that the solution of the recursion relation is

$$
H^{\mu} \sim J q^{\mu}+\mathcal{O}\left(z^{-1}\right),
$$

where the constant $J$ is $z$-independent. To obtain the amplitude from the current, it needs to be multiplied by the off-shell propagator $\left(p_{H}+p_{S}\right)^{2}$ that scales as $z$. Therefore, we find

$$
A^{\mu}(z) \sim z H^{\mu} \sim J z q^{\mu}+\mathcal{O}(1) .
$$

We use Eq. (7.50) in Eq. (7.46) and derive the scattering amplitude

$$
\mathcal{M}(z)=\epsilon_{1, \mu} A^{\mu}(z)=-\frac{p_{1}^{\mu} A^{\mu}(z)}{\sqrt{2} \mu z}=-\frac{J p_{1} \cdot q}{\sqrt{2} \mu}+\mathcal{O}\left(z^{-1}\right)=\mathcal{O}\left(z^{-1}\right)
$$


where the last step follows from the orthogonality of $p_{1}$ and $q$. Eq. (7.51) proves that the on-shell scattering amplitude vanishes in the limit $z \rightarrow \infty$. Finally, we note that, since the Berends-Giele recursion relation can be easily continued to higher-dimensional space-times, the above derivation shows that the BCFW relation Eq. (7.45), originally derived in four dimensions, remains valid in a space-time of arbitrary dimensionality.

\subsection{Computations with massive particles}

Unitarity-based computations are often discussed in the context of massless particles. Apart from well-known technical simplifications that are possible in massless theories, but are harder to achieve when massive particles are involved, there are important conceptual issues that must be understood before incorporating massive particle into the unitarity-based framework for loop computations. Potential complications can already be seen from the fact that when massive particles are involved, the full basis of master integrals includes tadpole integrals that do not have a discontinuity in any variable related to the external kinematics. However, when the generalized unitarity technique for one-loop computations is viewed as the consequence of the OPP procedure, it becomes perfectly clear that massive particles are straightforwardly incorporated into the unitarity framework. Nevertheless, peculiar features appear when unitarity and massive particles are combined; the goal of this Section is to mention them. In our discussion we closely follow Ref. [24]; we also note that all aspects of the following discussion have been checked in realistic computations of NLO QCD corrections to top quark pair production reported in Refs. [71, 72, 103]. Analytic computations of one-loop helicity amplitudes for $t \bar{t}$ production within generalized unitarity framework can be found in [106].

An important difference between computations with massless and massive particles is that in the former case there is no renormalization beyond the coupling constant. This happens because all one-particle reducible diagrams, usually associated with mass- and wave-function renormalization of external particles vanish in the massless case, provided that dimensional regularization is used. However, those quantities do not vanish in the massive case. If we define the renormalization constants for the quark mass $m$ and for the quark wave function $\psi$ in the on-shell scheme as

$$
m_{0}=Z_{m} m, \quad \psi_{0}=\sqrt{Z_{2}} \psi,
$$

in any covariant gauge we obtain

$$
Z_{m}=Z_{2}=1-C_{F} g_{s}^{2} c_{\Gamma}\left(\frac{\mu^{2}}{m^{2}}\right)^{\epsilon}\left(\frac{3}{\epsilon}+5-\eta\right) .
$$

We use parameter $\eta$ to distinguish between the four-dimensional helicity scheme $(\eta=0)$ and the 't Hooft-Veltman scheme $(\eta=1)$. We point out that the equality at one-loop between the two renormalization constants is fortuitous, since $Z_{m}$ has only ultraviolet divergences while $Z_{2}$ contains both ultraviolet and infrared divergences. 


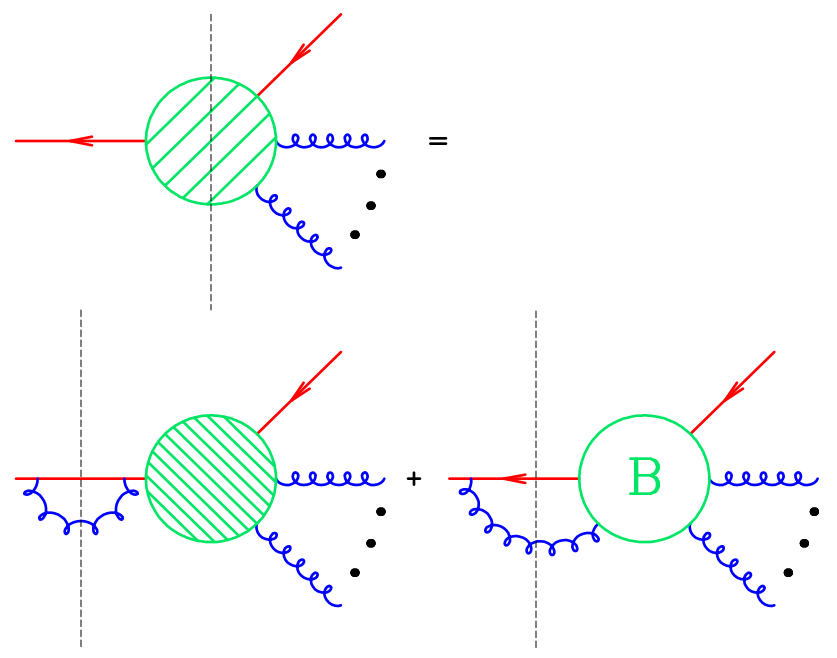

Figure 7.4: A particular cut of a general amplitude with massive quarks and gluons that splits it into a self-energy contribution and a higher-point function. The different shadings of the blobs represent different content.

The need to apply wave-function renormalization to remove ultraviolet divergences from a scattering amplitude is related to the fact that one-particle reducible self-energy corrections to external lines are non-vanishing. When oneloop scattering amplitudes are computed in a conventional diagrammatic framework, one-particle reducible diagrams are simply discarded, and their effect is accounted for by the wave-function renormalization constants. Disregarding one-particle reducible contributions is straightforward in the diagrammatic approach but it becomes more subtle, if the scattering amplitude is computed from unitarity cuts.

To illustrate this, we consider one-loop corrections to a scattering amplitude of a pair of massive quarks and any number of gluons. We focus on a particular double-cut of that scattering amplitude, where the heavy quark is on one side of the cut and every other particle is on the other side, see Fig. 7.4. The double cut of the one-loop amplitude is given by the product of two tree amplitudes,

$$
\operatorname{Res}[\mathcal{M}(q,\{q, g\})]=\sum_{\text {states }} \mathcal{M}_{0}\left(q, q^{*}, g^{*}\right) \mathcal{M}\left(q^{*}, g^{*},\{q, g\}\right) .
$$

The amplitude $\mathcal{M}_{0}$ describes the splitting of a massive fermion into an onshell fermion and a gluon; in turn, the amplitude $\mathcal{M}\left(q^{*}, g^{*},\{q, g\}\right)$ describes scattering of the quark $q^{*}$ and the gluon $g^{*}$ into the final state particles. On general grounds, this amplitude can be written as a sum of two terms

$$
\mathcal{M}\left(q^{*}, g^{*},\{q, g\}\right)=\frac{\left.R\left(p_{q^{*}}, p_{g^{*}},\{q, g\}\right)\right\}}{\left(p_{q^{*}}+p_{g^{*}}\right)^{2}-m^{2}}+B\left(p_{q^{*}}, p_{g^{*}},\{q, g\}\right),
$$

where the $B$-amplitude is non-singular in the limit $\left(p_{q^{*}}+p_{g^{*}}\right)^{2} \rightarrow m^{2}$. In the 
case of interest, momentum conservation forces the sum of the two momenta $p_{q^{*}}, p_{g^{*}}$ to be equal to $p_{q}$, so that $\left(p_{q^{*}}+p_{g^{*}}\right)^{2}=p_{q}^{2}=m^{2}$ and the first term in Eq. (7.55) becomes infinite. By reconstructing the diagram whose double cut corresponds to the product of $\mathcal{M}_{0}\left(q, q^{*}, g^{*}\right)$ and the singular term in Eq. (7.55), it is easy to understand that it corresponds to a one-particle reducible diagram - the self-energy insertion on an external massive fermion line.

In principle, when the one-loop amplitude is calculated recursively, it is easy to disregard the singular term by truncating the recursion steps. This corresponds to setting

$$
M\left(q^{*}, g^{*},\{q, g\}\right) \rightarrow B\left(p_{q^{*}}, p_{g^{*}},\{q, g\}\right)
$$

in Eq. (7.55). Such a replacement makes the matrix element finite but it introduces a problem since $B\left(p_{q^{*}}, p_{g^{*}},\{q, g\}\right)$ is not gauge-invariant. Gauge invariance is restored when the $B$-amplitude is combined with the on-shell wavefunction renormalization constant shown in Eq. (7.53). ${ }^{12}$ This requires that the $B$-amplitude and the wave function renormalization constant are computed in the same gauge. It is easiest to compute the wave-function renormalization constant in one of the covariant gauges; the result is given in Eq. (7.53). Hence, if we want to use $Z_{2}$ shown in Eq. (7.53) in an actual computation, we need to compute the $B$-amplitude also in a covariant gauge and we choose the Feynman gauge for that purpose. We implement the Feynman gauge in our calculation by introducing two unphysical polarizations for the "cut" gluon line with momentum $g^{*}$, so that the sum over polarizations reads

$$
\sum_{\lambda=1}^{4} \epsilon^{\mu}(\lambda) \epsilon^{\nu, *}(\lambda)=-g^{\mu \nu}
$$

We emphasize that this replacement should only be done for the computation of the $B$-amplitude, for a very special type of double cut. For other cuts, it is sufficient to sum over physical polarization states, thanks to gauge invariance of tree amplitudes.

Another important feature of calculations with massive internal particles is the existence of a non-vanishing double-cut contribution for light-like momenta. Because the square of the light-like momentum vanishes, the construction of the traditional van Neerven - Vermaseren basis becomes impossible. We have explained in the final paragraph of Sect. 5.2 how to proceed in this case; but we mention this feature here for completeness. Finally, we point out that when massive internal particles are present, single-particle cuts appear. While the calculation of those cuts is straightforward, it is important to keep in mind that also in that case one-particle reducible contributions to amplitudes cause trouble. The problematic contributions correspond to massive tadpoles diagrams

\footnotetext{
12 We note that the on-shell wave-function renormalization constant $Z_{2}$, computed in dimensional renormalization, is gauge-parameter independent in $R_{\xi}$-covariant gauges through two loops in QCD [107]. However, it is gauge-dependent since $Z_{2}$ in e.g. the light-cone gauge and $Z_{2}$ in the covariant gauges differ.
} 
and, just as is done in a diagrammatic computation, these contributions should be discarded. As we already pointed out in the context of the double-cut computation, within the unitarity framework this is accomplished by truncating steps in Berends-Giele recursion.

\section{Analytic computations}

In this Section we discuss some recent ideas related to the possibility to compute the reduction coefficients directly. We describe approaches suggested by Forde [29] and Mastrolia [31] to the computation of cut-constructible parts of one-loop amplitudes and by Badger [30] to the computation of the rational part. Our discussion is motivated by Refs. [29, 31, 30], but some details are different. In particular, in contrast to original references we do not use the spinor-helicity decomposition of the loop momentum. Furthermore, we attempt to provide an intuitive geometric picture behind the sophisticated choices of integration variables in Refs. $[29,31,30]$. In this respect, our discussion partially overlaps with the treatment of one-loop amplitudes in general quantum field theories, given in Ref. [108]. For simplicity, we consider only massless cases in this Section. The extension of the method of Ref. [29] to massive theories is given in Ref. [109].

\subsection{Direct computation of the cut-constructible coefficients}

To motivate the discussion that follows, we remind the reader that the cutconstructible part of any one-loop amplitude can be written as a linear combination of box, triangle, bubble and tadpole one-loop integrals. There is just one coefficient per master integral in such a linear combination but, within the OPP framework, we calculate two coefficients per box integral, seven coefficients per triangle, nine coefficients per bubble and five coefficients per tadpole. All but one of the coefficients for each master integral vanishes after integration over the loop momentum. Therefore, the very fact that those terms are computed, seems to imply that the OPP procedure is not as efficient as, perhaps, it is possible to make it. Of course, as should be clear from the discussion in preceding Sections, the large number of coefficients is computed on purpose since we want to subtract the full contributions of higher-point integrands to lowerpoint residues. Therefore, any inefficiency that may be present is not so easy to get rid of, but it is interesting to investigate if the computation of certain terms can be avoided. In this Section we explain, following Ref. [29], how to obtain the required minimal set of the reduction coefficients by simple algebraic manipulations with cuts of one-loop scattering amplitudes.

We begin with the easiest case - the reduction coefficients for four-point functions. As we explained above, there are two of them, but one vanishes upon integration over the loop momentum. Hence, we would like to avoid computing that coefficient. 
For definiteness, we consider the four-point master integral that contains inverse Feynman propagators $d_{0}, d_{1}, d_{2}, d_{3}$. The corresponding reduction coefficient is calculated from the quadruple cut [20]

$$
\tilde{d}_{0123}^{(0)}=\frac{1}{2} \sum_{i= \pm} A_{1}\left(l_{i}\right) A_{2}\left(l_{i}\right) A_{3}\left(l_{i}\right) A_{4}\left(l_{i}\right)
$$

where $A_{1, \ldots, 4}$ are the tree on-shell scattering amplitudes and $l_{ \pm}$are the two cut momenta that satisfy $d_{j}\left(l^{ \pm}\right)=0$, for all $j=0,1,2,3$. The result shown in Eq. (8.1) immediately follows from the discussion in the previous Section and, in particular, from Eq. (5.29). We stress that $\tilde{d}_{0123}^{(0)}$ is the only reduction coefficient that we need, to determine the contribution of a particular four-point function to a one-loop scattering amplitude.

Now, consider the triple-cut, that corresponds to the vanishing of the inverse propagators $d_{0}, d_{1}, d_{2}$. The triple cut is parametrized by seven reduction coefficients; only one of them does not vanish upon integration over the loop momentum. We now discuss the algebraic procedure that allows us to obtain the non-vanishing reduction coefficient directly.

As explained in Sect. 5.2, we can write the loop momentum on the cut as

$$
l^{\mu}=V^{\mu}+l_{\perp}\left(\cos \varphi n_{3}^{\mu}+\sin \varphi n_{4}^{\mu}\right) .
$$

We remind the reader that in this Section we only address the cut-constructible part. Therefore, the vector $l$ is purely four-dimensional and there is no dependence on $n_{\epsilon}$ in Eq. (8.2). In addition, we reiterate that we are treating the massless case, so the internal masses in the propagators are equal to zero. In Eq. (8.2), $V^{\mu}$ is a constant vector, given by a linear combination of vectors in the physical space (i.e. orthogonal to $n_{3}$ and $n_{4}$ that span the four-dimensional transverse space) and from $l^{2}=0$ it follows that $l_{\perp}=\sqrt{-V^{2}}$. Introducing $t_{+}=e^{i \varphi}$, we can rewrite Eq. (8.2) as

$$
l_{+}^{\mu}=V^{\mu}+l_{\perp}\left(t_{+} n_{-}^{\mu}+t_{+}^{-1} n_{+}^{\mu}\right),
$$

where $n_{\mp}=\left(n_{3} \mp i n_{4}\right) / 2$, so that $n_{-}^{2}=n_{+}^{2}=0$ and $2 n_{-} n_{+}=1$. The triple-cut of the one-loop amplitude, evaluated at the momentum $l=l_{+}$, reads

$$
A_{1}\left(t_{+}\right) A_{2}\left(t_{+}\right) A_{3}\left(t_{+}\right)=\tilde{c}_{012}\left(l_{+}\right)+\sum_{i=3}^{N} \frac{\tilde{d}_{012 i}^{(0)}+\tilde{d}_{012 i}^{(1)}\left(\tilde{n}_{i} \cdot l_{+}\right)}{d_{i}\left(l_{+}\right)},
$$

where $\tilde{n}_{i}$ is the four-vector orthogonal to $q_{1}, q_{2}$ and $q_{i}$. When writing Eq. (8.4) we used the fact the loop momentum in Eq. (8.3) depends on a single parameter $t_{+}$, therefore the tree amplitudes depend on the loop momentum only through $t_{+}$. The inverse propagator $d_{i}\left(l_{+}\right), i \in\{3, . ., N\}$, reads

$$
d_{i}\left(l_{+}\right)=\left(q_{i}+l_{+}\right)^{2}=\Delta_{i}+2 l_{\perp}\left(q_{i} \cdot n_{-}\right) t_{+}+2 l_{\perp}\left(q_{i} \cdot n_{+}\right) t_{+}^{-1},
$$

where $\Delta_{i}=q_{i}^{2}+2 q_{i} \cdot V$. Also, since $V^{\mu}$ is a linear combination of the propagator offset momenta $q_{1}^{\mu}$ and $q_{2}^{\mu}$ in $d_{1}$ and $d_{2}$ respectively and since $\tilde{n}_{i}^{\mu}$ is orthogonal 
to both of these momenta, we find

$$
\tilde{d}_{012 i}^{(0)}+\tilde{d}_{012 i}^{(1)}\left(\tilde{n}_{i} \cdot l_{+}\right)=\tilde{d}_{012 i}^{(0)}+l_{\perp} \tilde{d}_{012 i}^{(1)}\left[\left(\tilde{n}_{i} \cdot n_{-}\right) t_{+}+\left(\tilde{n}_{i} \cdot n_{+}\right) t_{+}^{-1}\right] .
$$

We use Eqs. $(8.5,8.6)$ to perform partial fractioning with respect to the variable $t_{+}$. We obtain

$$
\frac{\tilde{d}_{012 i}^{(0)}+\tilde{d}_{012 i}^{(1)}\left(\tilde{n}_{i} \cdot l_{+}\right)}{d_{i}\left(l_{+}\right)}=\tilde{d}_{012 i}^{(1)} \frac{\left(\tilde{n}_{i} \cdot n_{-}\right)}{\left(2 q_{i} \cdot n_{-}\right)}+\frac{r_{i, 1}}{t_{+}-t_{i}^{(1)}}+\frac{r_{i, 2}}{t_{+}-t_{i}^{(2)}}
$$

where $t_{i}^{(1)}, t_{i}^{(2)}$ are values of $t_{+}$for which $d_{i}\left(l_{+}\right)$vanishes and $r_{i, 1}, r_{i, 2}$ are independent of $t^{+}$.

The most general parametrization of the triangle residue $\tilde{c}_{012}\left(l_{+}\right)$is given in Eq. (5.16). We can set $n_{\epsilon} \rightarrow 0$ there since in this Section we are interested in the cut-constructible part only. If we write $\tilde{c}_{012}$ in terms of the variable $t_{+}$, we find

$$
\tilde{c}_{012}\left(l_{+}\right)=\tilde{c}_{012}^{(0)}+\sum_{k=-3, k \neq 0}^{3} c_{012}^{(k)} t_{+}^{k},
$$

where $\tilde{c}_{012}^{(0)}$ is the reduction coefficient of the three-point function. Putting box and triangle coefficients together, using Eqs. $(8.4,8.7,8.8)$, we derive the following decomposition of the triple cut of the integrand

$$
\begin{aligned}
A_{1}\left(t_{+}\right) A_{2}\left(t_{+}\right) A_{3}\left(t_{+}\right) & =\sum_{i=3}^{N}\left(\sum_{j=1}^{2} \frac{r_{i, j}}{t_{+}-t_{i}^{(j)}}+\tilde{d}_{012 i}^{(1)} \frac{\left(\tilde{n}_{i} \cdot n_{-}\right)}{\left(2 q_{i} \cdot n_{-}\right)}\right) \\
& +\tilde{c}_{012}^{(0)}+\sum_{k=-3, k \neq 0}^{k=3} c_{012}^{(k)} t_{+}^{k} .
\end{aligned}
$$

The question we address in the following is how to identify and extract the $\tilde{c}_{012}^{(0)}$ coefficient if the left hand side of Eq. (8.9) is analytically known as a function of $t$. To this end, we define an operator $\mathcal{L}_{t, m}$ such that when it acts on a rational function $F(t)$, it picks up the coefficient of the $\mathcal{O}\left(t^{m}\right)$ term in the Laurent expansion of $F(t)$ at $t=\infty .{ }^{13}$ Applying this operator to both sides of Eq. (8.9), we obtain

$$
\mathcal{L}_{t_{+}, 0}\left[A_{1}\left(t_{+}\right) A_{2}\left(t_{+}\right) A_{3}\left(t_{+}\right)\right]=\tilde{c}_{012}^{(0)}+\sum_{i=3}^{N} \tilde{d}_{012 i}^{(1)} \frac{\left(\tilde{n}_{i} \cdot n_{-}\right)}{\left(2 q_{i} \cdot n_{-}\right)} .
$$

Eq. (8.10) shows that we almost succeeded in computing the reduction coefficient of the triple-cut directly, except that in Eq. (8.10) there are still contributions from the evanescent coefficients of the quadruple residue, $\tilde{d}_{012 i}^{(1)}$, that need to be

${ }^{13}$ This operator is similar to the operator Inf introduced in Ref. [29]. 
removed. To accomplish this, we simply repeat the whole procedure taking the cut momentum in Eq. (8.2) to be

$$
l_{-}^{\mu}=V^{\mu}+l_{\perp}\left(t_{-} n_{-}^{\mu}+t_{-}^{-1} n_{+}^{\mu}\right),
$$

with $t_{-}=1 / t_{+}$. Following the discussion that leads to Eq. (8.2), with the obvious change $t_{+} \rightarrow t_{-}$where appropriate, we find

$$
\mathcal{L}_{t_{-}, 0}\left[A_{1}\left(t_{-}\right) A_{2}\left(t_{-}\right) A_{3}\left(t_{-}\right)\right]=\tilde{c}_{012}^{(0)}+\sum_{i=3}^{N} \tilde{d}_{012 i}^{(1)} \frac{\left(\tilde{n}_{i} \cdot n_{+}\right)}{\left(2 q_{i} \cdot n_{+}\right)} .
$$

Taking the average of Eqs. $(8.10,8.12)$, we obtain the final result for the triple cut coefficient

$$
\tilde{c}_{012}^{(0)}=\frac{1}{2} \sum_{i= \pm} \mathcal{L}_{t_{i}, 0}\left[A_{1}\left(t_{i}\right) A_{2}\left(t_{i}\right) A_{3}\left(t_{i}\right)\right] .
$$

In deriving Eq. (8.13), we used the identity

$$
\begin{aligned}
\frac{\left(\tilde{n}_{i} \cdot n_{-}\right)}{\left(q_{i} \cdot n_{-}\right)}+\frac{\left(\tilde{n}_{i} \cdot n_{+}\right)}{\left(q_{i} \cdot n_{+}\right)} & =\frac{\left(\tilde{n}_{i} \cdot n_{-}\right)\left(q_{i} \cdot n_{+}\right)+\left(\tilde{n}_{i} \cdot n_{+}\right)\left(q_{i} \cdot n_{-}\right)}{\left(q_{i} \cdot n_{-}\right)\left(q_{i} \cdot n_{+}\right)} \\
& =\frac{\tilde{n}_{i}^{\mu} \omega_{\mu \nu}\left(q_{1}, q_{2}\right) q_{i}^{\nu}}{2\left(q_{i} \cdot n_{-}\right)\left(q_{i} \cdot n_{+}\right)}=\frac{\tilde{n}_{i} \cdot q_{i}}{2\left(q_{i} \cdot n_{-}\right)\left(q_{i} \cdot n_{+}\right)}=0,
\end{aligned}
$$

where $\omega_{\mu \nu}\left(q_{1}, q_{2}\right)$ is the metric tensor of the transverse space orthogonal to vectors $q_{1}$ and $q_{2}$. Also, we note that in the last step in Eq. (8.14), we used the orthogonality of vectors $\tilde{n}_{i}$ and $q_{1}, q_{2}, q_{i \in[3, \ldots N]}$. Eq. (8.13) provides us with the local momentum-space relation between products of on-shell amplitudes on the triple cut and the reduction coefficient of the three-point function.

Next, we extend this discussion to double cuts. For definiteness, we consider a double cut specified by vanishing of two inverse Feynman propagators. In the massless case that we study here, these are given by $d_{0}=l^{2}$ and $d_{1}=\left(l+q_{1}\right)^{2}$. It follows from the discussion in the previous Section, that the general expression for the double cut is

$$
\left[A_{1} A_{2}\right]\left(l_{d}\right)=\tilde{b}_{01}\left(l_{d}\right)+\sum_{2 \leq i<j}^{N} \frac{\tilde{d}_{01 i j}\left(l_{d}\right)}{d_{i}\left(l_{d}\right) d_{j}\left(l_{d}\right)}+\sum_{i=2}^{N} \frac{\tilde{c}_{01 i}\left(l_{d}\right)}{d_{i}\left(l_{d}\right)},
$$

where $l_{d}$ is the momentum that satisfies the double-cut constraints $d_{0}\left(l_{d}\right)=0$, $d_{1}\left(l_{d}\right)=0$. Similar to the discussion of a triple-cut case, the goal is to find a procedure that allows the computation of the two-point function reduction coefficient $\tilde{b}_{01}^{(0)}$ without having to deal with many other terms present in Eq. (8.15). To motivate the loop momentum parametrization that we use for the double cut, we first discuss the genuine bubble coefficient $\tilde{b}_{01}\left(l_{d}\right)$ and ignore quadrupleand triple-cut remnants in Eq. (8.15).

As we explained in the previous Section, a generic parametrization of the loop momentum on the double-cut is

$$
l_{d}^{\mu}=-\frac{q_{1}^{\mu}}{2}+l_{\perp}\left(n_{2}^{\mu} \cos \theta+n_{3}^{\mu} \sin \theta \cos \varphi+n_{4}^{\mu} \sin \theta \sin \varphi\right),
$$


where $l_{\perp}=\sqrt{-q_{1}^{2}} / 2$. It is straightforward to rewrite this expression in terms of the variable $t=e^{i \varphi}$, that we introduced earlier. We obtain

$$
l_{d}^{\mu}=-\frac{q_{1}^{\mu}}{2}+l_{\perp}\left(n_{2}^{\mu} \cos \theta+t \sin \theta n_{-}^{\mu}+t^{-1} \sin \theta n_{+}^{\mu}\right) .
$$

To motivate the parametrization of $\cos \theta$ and $\sin \theta$ that we are about to introduce, we note that the full tensor structure of the bubble coefficient shown in Eq. (5.17) is a linear combination of the constant term, that we need, and spherical harmonics $Y_{l}^{m}(\theta, \varphi) \propto t^{m}$ with $l \leq 2$. We would like to find a way to identify and project out the spherical harmonics by working with rational functions. It is easy to realize that spherical harmonics with non-vanishing $m$ can be isolated by performing the Laurent expansion in the variable $t$ at $t=\infty$, and by picking up $t$-independent terms. Spherical harmonics that are not removed by this procedure correspond to $l=0, m=0, l=1, m=0$ and $l=2, m=0$. Hence, by performing Laurent expansion in $t$ and by picking up $\mathcal{O}\left(t^{0}\right)$ terms, we isolate ${ }^{14}$ the following structure on the double cut

$$
\tilde{b}_{01}\left(l_{d}\right) \Rightarrow b_{01}(\theta)=\tilde{b}_{01}^{(0)}+\tilde{b}_{01}^{(1)} \cos \theta+\tilde{b}_{01}^{(2)}\left(3 \cos ^{2} \theta-1\right) .
$$

We need a rational parametrization of $\cos \theta$ in terms of some variable, for which another Laurent expansion can be formulated. Typically, such parametrization will not lead to a rational parametrization of the $\sin \theta$ function in Eq. (8.17). For this reason, it is convenient to change variables $t \rightarrow z$, where $t=z / \sin \theta$, and use [29] $\cos \theta=1-2 y$ and $\sin ^{2} \theta=4 y(1-y)$. As a result, the momentum parametrization in Eq. (8.17) becomes

$$
l^{\mu}=-\frac{q_{1}^{\mu}}{2}+l_{\perp}\left(n_{2}^{\mu}(1-2 y)+z n_{-}^{\mu}+\frac{4 y(1-y)}{z} n_{+}^{\mu}\right) .
$$

The simplest way to extract the coefficient $\tilde{b}_{01}^{(0)}$ from the function $b_{01}(\theta)$ in Eq. (8.18) is to integrate over $\cos \theta$

$$
\frac{1}{2} \int_{-1}^{1} \mathrm{~d} \cos \theta b_{01}(\theta)=\tilde{b}_{01}^{(0)} .
$$

We would like to implement this integration as an algebraic procedure. To this end, note that because $-1<\cos \theta<1$, the integration region for $y$ is $0<y<1$, and the following integration rule is valid

$$
\int_{0}^{1} \mathrm{~d} y y^{m}=\frac{1}{m+1} \int_{0}^{1} \mathrm{~d} y .
$$

\footnotetext{
${ }^{14}$ To avoid confusion, we reiterate that we are are not discussing remnants of four- and three-point functions at the moment.
} 
Therefore, a substitution $y^{m} \rightarrow f_{m}=1 /(m+1)$, suggested in Ref. [29], is equivalent to integration over $\cos \theta$, which removes $\tilde{b}_{01}^{(1)} \cos \theta$ and $\tilde{b}_{01}^{(2)}\left(3 \cos ^{2} \theta-1\right)$ in Eq. (8.18). Hence, we find that if we use the parametrization Eq. (8.19) for the cut loop momentum, we arrive at the simple formula that extracts the reduction coefficient of the double-cut integral

$$
\tilde{b}_{01}^{(0)}=\left[\mathcal{L}_{z, 0}\left[\tilde{b}_{01}\left(l_{d}\right)\right]\right]^{y^{m} \rightarrow f_{m}} .
$$

In the above equation, the Laurent expansion in $z$ removes all the spherical harmonics with non-trivial dependence on the azimuthal angle $\varphi$, while the substitutions $y^{m} \rightarrow f_{m}$ help us to integrate over $\cos \theta$, removing contributions of $Y_{l=1,2}^{0}(\cos \theta)$ and leaving the constant term $\tilde{b}_{01}^{(0)}$ only. Note that one can accomplish the same goal in a slightly different way, by assuming the following parametrization for $\cos \theta$

$$
\cos \theta=\frac{1}{2}\left(w+\frac{2}{3 w}\right) .
$$

This parametrization has the property that $w$-independent term drops out both in $\cos \theta$ and in $3 \cos ^{2} \theta-1$. This implies that the term in the Laurent expansion that scales like $\mathcal{O}\left(w^{0} z^{0}\right)$ is the reduction coefficient of a two-point function $\tilde{b}_{01}^{(0)}$; no integration over any parameter is required.

According to Ref. [29], the full double-cut reduction coefficient can be calculated with the help of the following equation

$$
\begin{aligned}
b_{01}^{(0)} & =\left[\mathcal{L}_{z, 0}\left[\mathcal{L}_{y, \geq 0}\left[A_{1} A_{2}\right]^{y^{m} \rightarrow f_{m}}\right]\right] \\
& -\frac{1}{2} \sum_{i, \alpha= \pm}\left[\mathcal{L}_{z, \geq 0}\left[A_{1} A_{2} A_{3}\right]^{(i)}\left(z, y_{\alpha}^{(i)}\right)\right]^{z^{n} \rightarrow Z(n)},
\end{aligned}
$$

where the sum runs over all propagators $d_{i}$ that, when taken together with $d_{0}$ and $d_{1}$, make a valid triple cut of the one-loop amplitude. Also, $f_{m}=1 /(m+1)$, and $Z(n), n=0,1,2,3$ are functions of external momenta and the integer $n$; they can be found in Ref. [29] and we also derive them in what follows. The Laurent expansion operator $\mathcal{L}_{x, \geq 0}$ implies that after performing the Laurent expansion in the variable $x$ at infinity, only non-negative powers of that variable must be kept. It is assumed that the momentum parametrization of Eq. (8.19) is used in Eq. (8.24) and $y_{ \pm}^{(i)}$ are the two values of the variable $y$ for which $d_{i}\left(l_{d}\left(z, y_{ \pm}^{(i)}\right)\right)=0$.

We would like to prove the validity of Eq. (8.24). Since the most general parametrization of the double and triple cuts is provided by the OPP parametrization of the residues, we can check Eq. (8.24) directly. We first explain why remnants of the quadruple cuts (the terms $\tilde{d}_{01 i j}\left(l_{d}\right) / d_{i}\left(l_{d}\right) / d_{j}\left(l_{d}\right)$ ) do not contribute to Eq. (8.24). It happens for two different reasons. One such contribution is removed by an operator $\mathcal{L}_{y, \geq 0}$ from the products of two on-shell amplitudes $A_{1} A_{2}$ in Eq. (8.24), because by simple power counting remnants 
of the quadruple cuts vanish in the large $y$-limit. Interestingly, in the case of products of three on-shell amplitudes $A_{1} A_{2} A_{3}$, remnants of quadruple cuts produce finite $z$-independent terms, besides terms that vanish in the large- $z$ limit. However, as we explain below, we have to set $Z(0)=0$, for consistency; this completely removes contributions of quadruple cuts from the right hand side Eq. (8.24).

We turn to the discussion of double and triple cuts contributions to Eq. (8.24). The relevant expression for the double cut is given in Eq. (8.15). Since we explained that the quadruple cut contributions are immaterial, the product of three on-shell amplitudes is given by the $c(l)$-functions, evaluated with the double-cut loop momentum. Since $\tilde{b}_{01}\left(l_{d}\right)$ is a polynomial in $y$, we can use the method explained in the previous Section to extract $\tilde{b}_{01}^{(0)}$. We find

$$
\mathcal{L}_{z, 0}\left[\mathcal{L}_{y, \geq 0}\left[\tilde{b}_{01}\left(l_{d}\right)\right]^{y^{m} \rightarrow f_{m}}\right]=\tilde{b}_{01}^{(0)}
$$

We apply $\mathcal{L}_{z, 0}[\ldots]^{y^{m} \rightarrow f_{m}}$ to $A_{1} A_{2}$ in Eq. (8.15). As discussed quadruple cuts do not contribute, therefore Eq. (8.24) can only be valid if the triple-cut functions satisfy the equation

$$
\begin{aligned}
\mathcal{L}_{z, 0}\left[\mathcal{L}_{y, \geq 0}\right. & {\left.\left[\frac{\tilde{c}_{01 i}\left(l_{d}(z, y)\right)}{d_{i}\left(l_{d}\right)}\right]^{y^{m} \rightarrow f_{m}}\right]=} \\
& \frac{1}{2}\left[\mathcal{L}_{\mathrm{z}, \geq 0}\left[\tilde{c}_{01 i}\left(l_{d}\left(z, y_{+}^{i}\right)\right)+\tilde{c}_{01 i}\left(l_{d}\left(z, y_{-}^{i}\right)\right)\right]\right]^{z^{n} \rightarrow Z(n)},
\end{aligned}
$$

for each value of $i$. In general, $\tilde{c}_{01 i}(l)$ is the rank-three tensor but, for the sake of simplicity, we will begin by considering it to be rank-two. We comment on the rank-three contributions to $\tilde{c}_{01 i}(l)$ at the end of this Section. We write

$$
\tilde{c}_{01 i}(l)=\tilde{c}_{01 i}^{(0)}+s_{\mu} l^{\mu}+t_{\mu \nu} l^{\mu} l^{\nu}
$$

where $s^{\mu}$ is a vector and $t^{\mu \nu}$ is a traceless tensor that are both transverse to $q_{1}$ and $q_{i}$.

As a first step, we compute the left hand side in Eq. (8.26). We perform a Laurent expansion in $y$ at infinity, keeping non-negative powers in the expansion. We integrate over $y$ from zero to one, which is equivalent to making the substitutions $y^{m} \rightarrow 1 /(m+1)$. Then, we perform a further Laurent expansion in $z$ at $z=\infty$, and pick up the $z$-independent term $\mathcal{O}\left(z^{0}\right)$. Finally, we obtain a simple expression

$$
\mathcal{L}_{z, 0}\left[\mathcal{L}_{y, \geq 0}\left[\frac{c_{i}\left(l_{d}(z, y)\right)}{d_{i}\left(l_{d}\right)}\right]^{y^{m} \rightarrow f_{m}}\right]=\frac{1}{2 u_{+}} s \cdot n_{+}-\frac{\Delta_{i}}{4 u_{+}^{2}} t_{\mu \nu} n_{+}^{\mu} n_{+}^{\nu},
$$

where $\Delta_{i}=q_{i}^{2}-q_{i} \cdot q_{1}$ and $u_{+}=q_{i} \cdot n_{+}$. We now check if a similar expression can be obtained by computing the right hand side of Eq. (8.26). To do that, we need the double-cut momenta evaluated at two particular values of the variable 
$y$, namely $y=y_{ \pm}^{(i)}$. It is convenient to introduce two auxiliary vectors $L=$ $\left(l\left(z, y_{+}\right)+l\left(z, y_{-}\right)\right) / 2$ and $K=\left(l\left(z, y_{+}\right)-l\left(z, y_{-}\right)\right) / 2$. After some algebra, we find

$$
L^{\mu}=-\frac{q_{1}^{\mu}}{2}+l_{\perp}\left(-\frac{\Delta_{i}}{2 l_{\perp} u_{+}} n_{+}^{\mu}+\frac{z}{2 u_{+}} \omega^{\mu \nu}\left(q_{1}\right) q_{i, \nu}-z \frac{q_{i}^{\alpha} \omega_{\alpha \beta}\left(q_{1}\right) q_{i}^{\beta}}{2 u_{+}^{2}} n_{+}^{\mu}\right),
$$

where $\omega_{\mu \nu}\left(q_{1}\right)$ is the metric tensor of the three-dimensional space which is transverse to the vector $q_{1}$. In deriving Eq. (8.29), it is important to use the completeness relations for vectors $n_{2}, n_{ \pm}$

$$
2 n_{+}^{\mu} n_{-}^{\nu}+2 n_{-}^{\mu} n_{+}^{\nu}+n_{2}^{\mu} n_{2}^{\nu}=\omega^{\mu \nu}\left(q_{1}\right)=g^{\mu \nu}-\frac{q_{1}^{\mu} q_{1}^{\nu}}{q_{1}^{2}} .
$$

Because we will only use the vector $L^{\mu}$ in formulas where it is contracted with vectors or tensors that are transverse to $q_{1}$ and $q_{i}$, we can drop $q_{1}^{\mu}$ and $\omega^{\mu \nu}\left(q_{1}\right) q_{i, \nu}$ in Eq. (8.29). Upon doing that, we get a very simple expression

$$
L^{\mu} \rightarrow L^{\mu}=-\frac{n_{+}^{\mu}}{2 u_{+}}\left(\Delta_{i}+z \frac{q_{i, \perp}^{2} l_{\perp}}{u_{+}}\right),
$$

where $q_{i, \perp}^{2}=q_{i}^{\alpha} \omega_{\alpha \beta}\left(q_{1}\right) q_{i}^{\beta}$. The vector $K_{\mu}$ is obtained using similar arguments. We find

$$
K^{\mu}=l_{\perp}\left(y_{-}-y_{+}\right) \kappa^{\mu}, \quad \kappa^{\mu}=n_{2}^{\mu}-\frac{u_{2}}{u_{+}} n_{+}^{\mu},
$$

where $u_{2}=q_{i} \cdot n_{2}$. It is easy to establish that only an even number of vectors $K_{\mu}$ can enter the computation (as can be seen explicitly from Eq. (8.34); as a result, we need

$$
\left(y_{-}-y_{+}\right)^{2}=1+\frac{q_{i, \perp}^{2}}{4 u_{+}^{2}} z^{2}+\frac{\Delta_{i} z}{2 l_{\perp} u_{+}} .
$$

We are now in position to compute $\bar{c}_{i}=\tilde{c}_{01 i}\left(z, y_{+}\right) / 2+\tilde{c}_{01 i}\left(z, y_{-}\right) / 2$. We obtain

$$
\begin{aligned}
& \bar{c}=\tilde{c}_{01 i}^{(0)}+s_{\mu} L^{\mu}+t_{\mu \nu} L^{\mu} L^{\nu}+t_{\mu \nu} K^{\mu} K^{\nu} \\
& =\tilde{c}_{01 i}^{(0)}-\frac{s_{\mu} n_{+}^{\mu}}{2 u_{+}}\left(\Delta_{i}+z \frac{q_{i, \perp}^{2} l_{\perp}}{u_{+}}\right)+t_{\mu \nu} \frac{n_{+}^{\mu} n_{+}^{\nu}}{4 u_{+}^{2}}\left(\Delta_{i}+z \frac{q_{i, \perp}^{2} l_{\perp}}{u_{+}}\right)^{2} \\
& +l_{\perp}^{2}\left(1+\frac{q_{i, \perp}^{2}}{4 u_{+}^{2}} z^{2}+\frac{\Delta_{i} z}{2 l_{\perp} u_{+}}\right) t_{\mu \nu} \kappa^{\mu} \kappa^{\nu},
\end{aligned}
$$

which implies that $\bar{c}$ is a polynomial in $z$. Forde [29] suggests to match Eq. (8.34) and Eq. (8.28) by defining mappings of powers of $z$ on to some functions $z^{n} \rightarrow$ $Z(n)$. Since linearly independent tensor structures must satisfy such mappings separately, the possibility to do that is not obvious. We now show that it is possible. 
To construct such a mapping, we note that Eq. (8.28) does not contain $\tilde{c}_{01 i}^{(0)}$, while Eq. (8.34) does. This suggests that all $z$-independent terms in Eq. (8.34) must be set to zero, $z^{0} \rightarrow Z(0)=0$; Eq. (8.34) becomes

$$
\begin{aligned}
\bar{c}= & -s_{\mu} n_{+}^{\mu} \frac{z q_{i, \perp}^{2} l_{\perp}}{2 u_{+}^{2}}+t_{\mu \nu} \frac{n_{+}^{\mu} n_{+}^{\nu}}{4 u_{+}^{2}}\left(z \frac{2 \Delta_{i} q_{i, \perp}^{2} l_{\perp}}{u_{+}}+\frac{z^{2} q_{i, \perp}^{4} l_{\perp}^{2}}{u_{+}^{2}}\right) \\
& +l_{\perp}^{2}\left(\frac{q_{i, \perp}^{2}}{4 u_{+}^{2}} z^{2}+\frac{\Delta_{i} z}{2 l_{\perp} u_{+}}\right) t_{\mu \nu} \kappa^{\mu} \kappa^{\nu} .
\end{aligned}
$$

Comparing Eq. (8.35) and Eq. (8.28), we see that expressions for the rankone tensors match, provided that we make the substitution

$$
z \rightarrow Z(1)=-\frac{u_{+}}{l_{\perp} q_{i, \perp}^{2}}
$$

As a result, Eq. (8.35) becomes

$$
\bar{c}=\frac{s_{\mu} n_{+}^{\mu}}{2 u_{+}}+\left(\frac{z^{2} q_{i, \perp}^{4} l_{\perp}^{2}}{u_{+}^{2}}-2 \Delta_{i}\right) t_{\mu \nu}\left(\frac{n_{+}^{\mu} n_{+}^{\nu}}{4 u_{+}^{2}}+\frac{\kappa^{\mu} \kappa^{\nu}}{4 q_{i, \perp}^{2}}\right) .
$$

When we compare tensor structures in Eq. (8.37) and Eq. (8.28), there appears to be a problem because in Eq. (8.28) the tensor structure involves $n_{+}^{\mu} n_{+}^{\nu}$ but in Eq. (8.37) $\kappa^{\mu} \kappa^{\nu}$ appears in addition. The two equations get reconciled if we use properties of the tensor $t_{\mu \nu}$ and the vectors $n_{+}^{\mu}$ and $\kappa^{\mu}$.

First, we note that $t_{\mu \nu}$ is symmetric, traceless rank-two tensor in the space that is transverse to $q_{1}$ and $q_{i}$. Because vector $\kappa$ satisfies $\kappa \cdot q_{i}=\kappa \cdot q_{1}=0$, $\kappa^{2}=1$, we can use it as one of the basis vectors of the required transverse space. We call the other basis vector $\sigma, \sigma^{2}=1$. Then we write the most general parametrization for the tensor $t_{\mu \nu}$

$$
t_{\mu \nu}=t_{1}\left(\sigma_{\mu} \sigma_{\nu}-\kappa_{\mu} \kappa_{\nu}\right)+\frac{t_{2}}{2}\left(\sigma_{\mu} \kappa_{\nu}+\kappa_{\nu} \sigma_{\mu}\right) .
$$

When contracting $t_{\mu \nu}$ with $n_{+}^{\mu} n_{+}^{\nu}$ and $\kappa^{\mu} \kappa^{\nu}$, we use the fact that $\kappa \cdot n_{+}=0$, $\kappa \cdot \sigma=0$. Also, we need $\left(\sigma \cdot n_{+}\right)^{2}$ and we compute it using the completeness identity

$$
\omega^{\mu \nu}\left(q_{1}, q_{i}\right)=\kappa^{\mu} \kappa^{\nu}+\sigma^{\mu} \sigma^{\nu}
$$

Contracting $\omega^{\mu \nu}$ with $n_{+}^{\mu} n_{+}^{\nu}$, we obtain

$$
\left(\sigma \cdot n_{+}\right)^{2}=n_{+}^{\mu} \omega_{\mu \nu}\left(q_{1}, q_{i}\right) n_{+}^{\nu}=-\frac{u_{+}^{2}}{q_{i, \perp}^{2}} .
$$

Putting everything together, we arrive at

$$
t_{\mu \nu}\left(\frac{n_{+}^{\mu} n_{+}^{\nu}}{4 u_{+}^{2}}+\frac{\kappa^{\mu} \kappa^{\nu}}{4 q_{i, \perp}^{2}}\right)=t_{1}\left(\frac{\left(\sigma \cdot n_{+}\right)^{2}}{4 u_{+}^{2}}-\frac{1}{4 q_{i, \perp}^{2}}\right)=-\frac{t_{1}}{2 q_{i, \perp}^{2}} .
$$


We then find

$$
\bar{c}=\frac{s_{\mu} n_{+}^{\mu}}{2 u_{+}}-\left(\frac{z^{2} q_{i, \perp}^{4} l_{\perp}^{2}}{u_{+}^{2}}-2 \Delta_{i}\right) \frac{t_{1}}{2 q_{i, \perp}^{2}} .
$$

Requiring that Eq. (8.42) matches Eq. (8.28), we find the substitution rule

$$
z^{2} \rightarrow Z(2)=\frac{3 \Delta_{i} u_{+}^{2}}{2 q_{i, \perp}^{4} l_{\perp}^{2}}
$$

Finally, we discuss an extension of these results to the rank-three case. Consider the additional term in Eq. (8.27)

$$
\tilde{c}_{01 i}(l) \rightarrow \tilde{c}_{01 i}(l)+t^{\mu \nu \alpha} l_{\mu} l_{\nu} l_{\alpha} .
$$

When $t^{\mu \nu \alpha}$ is expressed through basis vectors $\kappa$ and $\sigma$, four terms appear

$$
t^{\mu \nu \alpha}=a_{1} \omega^{\{\mu \nu} \kappa^{\alpha\}}+a_{2} \omega^{\{\mu \nu} \sigma^{\alpha\}}+a_{3} \kappa^{\mu} \kappa^{\nu} \kappa^{\alpha}+a_{4} \sigma^{\mu} \sigma^{\nu} \sigma^{\alpha} .
$$

In Eq. (8.45), we introduce the metric tensor of the vector space transverse to $q_{1}$ and $q_{i}, \omega_{\mu \nu}=\omega_{\mu \nu}\left(q_{1}, q_{i}\right)$. Also, indices in curly brackets need to be symmetrized. Computations in this case are straightforward, although more tedious than for the rank-one and rank-two. We skip all the details and only present the result. First, the Laurent expansion, restricted to the rank three terms, gives

$$
\begin{aligned}
\mathcal{L}_{z, 0}\left[\mathcal{L}_{y, \geq 0}\left[\frac{c_{i}\left(l_{d}\right)}{d_{i}\left(l_{d}\right)}\right]^{y^{m} \rightarrow f_{m}}\right] & =a_{2}\left(\sigma \cdot n_{+}\right)\left(-\frac{3 \Delta_{i}^{2}}{8 q_{i, \perp}^{2} u_{+}}+\frac{3 l_{\perp}^{2}}{2 u_{+}}\right) \\
& +a_{4}\left(\sigma \cdot n_{+}\right)\left(-\frac{\Delta_{i}^{2}}{8 q_{i, \perp}^{2} u_{+}}+\frac{l_{\perp}^{2}}{3 u_{+}}\right) .
\end{aligned}
$$

Second, calculating the function $\tilde{c}_{01 i}$ for $l_{d}\left(z, y_{ \pm}\right)$, and using the substitution rules for $z^{n} \rightarrow Z(n), n=0,1,2$, that we derived in this Section, we obtain

$$
\begin{aligned}
\frac{1}{2}\left(c\left(z, y_{+}\right)+c\left(z, y_{-}\right)\right) & =a_{2}\left(\sigma \cdot n_{+}\right)\left(-\frac{3 \Delta_{i}^{2}}{8 q_{i, \perp}^{2} u_{+}}+\frac{3 l_{\perp}^{2}}{2 u_{+}}\right) \\
& +a_{4}\left(\sigma \cdot n_{+}\right)\left(\frac{3 \Delta_{i}^{2}}{16 q_{i, \perp}^{2} u_{+}}+\frac{z^{3} l_{\perp}^{3} q_{\perp, i}^{4}}{8 u_{+}^{4}}\right) .
\end{aligned}
$$

Comparing Eq. (8.46) and Eq. (8.47), we find that they can be matched by the substitution

$$
z^{3} \rightarrow Z(3)=\frac{8 u_{+}^{3}}{3 l_{\perp} q_{i, \perp}^{4}}-\frac{5 \Delta_{i}^{2} u_{+}^{3}}{2 q_{i, \perp}^{6} l_{\perp}^{3}} .
$$

Because the highest rank of a tensor in the function $c(l)$ is three, Eq. (8.48) completes the list of substitutions that are required to prove the validity of Eqs. (8.24) and (8.26). 


\subsection{An alternative formula for bubble reduction coefficients}

In this Section we describe another approach to the calculation of bubble reduction coefficients, suggested by Mastrolia in Ref. [31]. We consider a doublecut of the one-loop scattering amplitude that is defined by the condition that two inverse propagators, $d_{0}$ and $d_{1}$, vanish. We use the parametrization of the loop momentum given in Eq. (8.16), take the double-cut of the amplitude given in Eq. (8.15), and integrate over $\theta$ and $\varphi$. We use the expression for the coefficients $\tilde{d}(l), \tilde{c}(l), \tilde{b}(l)$ in Eqs. $(5.12,5.16,5.17)$, neglect terms proportional to $n_{\epsilon}$, since here we are interested in the cut-constructible part only, and drop terms that vanish after the integration over the solid angle. We obtain

$$
\int \frac{\mathrm{d} \Omega}{4 \pi}\left[A_{1} A_{2}\right]\left(l_{d}\right)=\tilde{b}_{01}^{(0)}+\sum_{i} \int \frac{\mathrm{d} \Omega}{4 \pi} \frac{\tilde{c}_{01 i}^{(0)}}{d_{i}\left(l_{d}\right)}+\sum_{i j} \int \frac{\mathrm{d} \Omega}{4 \pi} \frac{\tilde{d}_{01 i j}^{(0)}}{d_{i}\left(l_{d}\right) d_{j}\left(l_{d}\right)},
$$

where $\mathrm{d} \Omega=\mathrm{d} \cos \theta \mathrm{d} \varphi$ is an element of the solid angle. We stress that remnants of three- and four-point functions in the right hand side of Eq. (8.49) are multiplied by the corresponding reduction coefficients $\tilde{c}_{01 i}^{(0)}$ and $d_{01 i j}^{(0)}$ and not by the full $l$-dependent functions $c(l)$ and $d(l)$. We now rewrite the integration over the solid angle by performing the standard change of variables

$$
\rho=\tan \frac{\theta}{2}
$$

to express $\cos \theta, \sin \theta$ as rational functions of $\rho$

$$
\cos \theta=\frac{1-\rho^{2}}{1+\rho^{2}}, \quad \sin \theta=\frac{2 \rho}{1+\rho^{2}}, \quad \tan \theta=\frac{2 \rho}{1-\rho^{2}} .
$$

We further introduce a complex variable $z=\rho e^{i \varphi}$, denote $\bar{z}=\rho e^{-i \varphi}$, and write

$$
\cos \theta=\frac{1-z \bar{z}}{1+z \bar{z}}, \quad \tan \theta e^{i \varphi}=\frac{2 z}{1-z \bar{z}}, \quad \tan \theta e^{-i \varphi}=\frac{2 \bar{z}}{1-z \bar{z}} .
$$

The final parametrization of the loop momentum reads

$$
l_{d}^{\mu}=-\frac{q_{1}^{\mu}}{2}+\frac{\sqrt{-q_{1}^{2}}}{2} \frac{1-z \bar{z}}{1+z \bar{z}}\left(n_{2}^{\mu}+\frac{2 z}{1-z \bar{z}} n_{-}^{\mu}+\frac{2 \bar{z}}{1-z \bar{z}} n_{+}^{\mu}\right) .
$$

Given the mapping between $\theta, \varphi$ and $z, \bar{z}$ variables, it is easy to find the relation of the integration measures

$$
\mathrm{d} \Omega=\frac{2 \mathrm{~d} \bar{z} \wedge \mathrm{d} z}{i(1+z \bar{z})^{2}} .
$$

Integration over $z$ and $\bar{z}$ extends through the entire complex plane $D_{\infty}$. We therefore rewrite Eq. (8.49) as

$$
\tilde{b}_{01}^{(0)}=\frac{1}{2 \pi i} \int_{D_{\infty}} \frac{\mathrm{d} \bar{z} \wedge \mathrm{d} z}{(1+z \bar{z})^{2}} f(z, \bar{z})
$$


where

$$
f(z, \bar{z})=\left[A_{1} A_{2}\right]\left(l_{d}\right)-\sum_{i} \frac{\tilde{c}_{01 i}^{(0)}}{d_{i}\left(l_{d}\right)}-\sum_{i j} \frac{\tilde{d}_{01 i j}^{(0)}}{d_{i}\left(l_{d}\right) d_{j}\left(l_{d}\right)}
$$

and the cut momentum $l_{d}$ is given by Eq. (8.53).

As recognized in Ref. [31], Eq. (8.55) has a structure that can be integrated using the generalized Cauchy (or Cauchy-Pompeiu) theorem. The theorem states that for a rational function $F(z, \bar{z})$ defined in a domain $D_{c}$ of a complex plane that is bounded by a contour $L_{c}$, the following identity is valid

$$
\begin{gathered}
\frac{1}{2 \pi i} \oint_{L_{c}} d z F(z, \bar{z})-\frac{1}{2 \pi i} \int_{D_{c}} \frac{\partial F(z, \bar{z})}{\partial \bar{z}} \mathrm{~d} \bar{z} \wedge \mathrm{d} z \\
=\sum \operatorname{Res}(F(z, \bar{z})) .
\end{gathered}
$$

The sum on the right-hand side of Eq. (8.57) runs over all the $z$-poles in the domain $D_{c}$. To apply this theorem to the calculation of the double-cut coefficient, we identify

$$
\frac{\partial F(z, \bar{z})}{\partial \bar{z}}=\frac{f(z, \bar{z})}{(1+z \bar{z})^{2}} .
$$

We also identify $D_{c}$ with $D_{\infty}$; as a consequence, the contour $L_{c}$ in Eq. (8.57) runs at infinity. Because the limit $z, \bar{z} \rightarrow \infty$ corresponds to finite cut-momenta $l_{d}$ (see Eq. (8.53)), the function $f(z, \bar{z})$ remains finite in that limit. Then, as a consequence of Eq. (8.58), we can choose the function $F(z, \bar{z})$ such that it vanishes at the complex infinity as $1 /\left(z^{2} \bar{z}\right)$. Hence the first term in the CauchyPompeiu formula Eq. (8.57) can be dropped and we find

$$
\sum_{z \text { poles } \in D_{\infty}} \operatorname{Res}[F(z, \bar{z})]=-\frac{1}{2 \pi i} \int_{D_{c}} \frac{\partial F(z, \bar{z})}{\partial \bar{z}} \mathrm{~d} \bar{z} \wedge \mathrm{d} z=-\tilde{b}_{01}^{(0)},
$$

where in the last equation we simply inserted Eq. (8.55).

It follows from Eq. (8.59) that, to calculate the coefficient $\tilde{b}_{01}^{(0)}$, we need to find the anti-derivative function $F(z, \bar{z})$ and compute its residues in the entire complex plane. According to Eqs. $(8.56,8.58)$, the function $F(z, \bar{z})$ can be written as a sum of three terms

$$
F(z, \bar{z})=F^{(A)}(z, \bar{z})-\sum_{i} F^{\left(c_{i}\right)}(z, \bar{z})-\sum_{i j} F^{\left(d_{i j}\right)}(z, \bar{z}),
$$

where

$$
\frac{\partial F^{(A)}(z, \bar{z})}{\partial \bar{z}}=(1+z \bar{z})^{-2}\left[A_{1} A_{2}\right](z, \bar{z})
$$

and $F^{\left(c_{i}\right),\left(d_{i j}\right)}$ are anti-derivatives due to contributions of three- and four-point functions in Eq. (8.56). It is easy to see that, $F^{\left(c_{i}\right),\left(d_{i j}\right)}$ have the following form

$$
\operatorname{Res}\left[F^{(c, d)}(z, \bar{z})\right] \sim R(z, \bar{z}) \ln (Q(z, \bar{z})),
$$


where $R(z, \bar{z})$ and $Q(z, \bar{z})$ are some rational functions. Those terms subtract similar contributions to function $F^{(A)}(z, \bar{z})$; as a result they do not affect the evaluation of the double-cut reduction coefficient $\tilde{b}_{01}^{(0)}$. This observation was used in Ref. [31] where it was suggested that one can drop $F^{(c, d)}(z, \bar{z})$ and all the logarithmic terms in $F^{(A)}(z, \bar{z})$ to obtain a simpler formula for the reduction coefficient

$$
\tilde{b}_{01}^{(0)}=-\sum_{z \text { poles } \in D_{\infty}} \operatorname{Res}\left[F^{(A), \text { rat }}(z, \bar{z})\right] .
$$

In Eq. (8.63) $F^{(A), \text { rat }}(z, \bar{z})$ is the anti-derivative as in Eq. (8.61), from where all the logarithmic terms are omitted.

There is a subtlety in proving Eq. (8.63), which is not mentioned in the literature. Indeed, consider a representation of the amplitudes $A_{1} A_{2}$ on a double cut in terms of the OPP reduction coefficients

$$
\left[A_{1} A_{2}\right]\left(l_{d}\right)=\tilde{b}_{01}\left(l_{d}\right)+\sum \frac{\tilde{c}_{0 i 1}\left(l_{d}\right)}{d_{i}\left(l_{d}\right)}+\ldots
$$

Using the explicit parametrization of the cut momentum $l_{d}$ as in Eq. (8.53), it is straightforward to prove that $\tilde{b}_{01}\left(l_{d}\right)$ only contributes to $F^{(A) \text {,rat }}(z, \bar{z})$. The term $\tilde{c}_{01 i}^{(0)} / d_{i}$ contributes to the logarithmic part of $F^{(A)}(z, \bar{z})$ and hence it is discarded when $F^{(A) \text {,rat }}$ is constructed.

A more complicated situation occurs however with the spurious contributions to $\tilde{c}_{01 i}\left(l_{d}\right)$. Consider the rank-one tensor $\tilde{c}_{01 i}^{(1)}\left(s \cdot l_{d}\right) / d_{i}\left(l_{d}\right)$ as an example. Using the parametrization for $d_{i}\left(l_{d}\right)$ in terms of $z, \bar{z}$, we write

$$
d_{i}(z, \bar{z})=\frac{P_{12}(z)+\bar{z} P_{34}(z)}{1+z \bar{z}},
$$

where $P_{12}(z)=p_{1}+z p_{2}, P_{34}(z)=p_{3}+z p_{4}$ and

$$
\begin{aligned}
& p_{1}=q_{i}^{2}-q_{i} \cdot q_{1}+2 l_{\perp} n_{2} \cdot q_{i}, \quad p_{2}=4 l_{\perp} n_{-} \cdot q_{i}, \\
& p_{3}=4 l_{\perp} n_{+} \cdot q_{i}, \quad p_{4}=q_{i}^{2}-q_{i} \cdot q_{1}-2 l_{\perp} n_{2} \cdot q_{i} .
\end{aligned}
$$

Computing the contribution of those terms to $F^{(A)}(z, \bar{z})$ we find

$$
F_{c_{i}^{(1)}}^{(A)}(z, \bar{z})=\frac{N(z, \bar{z}) \ln \left(d_{i}(z, \bar{z})\right)}{\left(P_{12}(z) z-P_{34}(z)\right)^{2}}+R(z, \bar{z}) .
$$

The second power of the rational function in the denominator of the logarithmic term in the above equation is a direct consequence of the fact that we are dealing with the rank-one tensor. In general, a rank- $n$ tensor integral leads to the appearance of terms $\ln \left[d_{i}(z, \bar{z})\right] /\left(P_{12}(z) z-P_{34}(z)\right)^{n+1}$ in the anti-derivative.

Because our original expression is a spurious term, it should integrate to zero. This implies that the following equation holds

$$
\sum_{z \text { poles } \in D_{\infty}} \operatorname{Res}\left[F_{c_{i}^{(1)}}^{(A)}(z, \bar{z})\right]=0 .
$$


Writing $P_{12}(z) z-P_{34}(z)=p_{2}\left(z-z_{+}\right)\left(z-z_{-}\right)$and computing residues at, say, $z=z_{+}$, we find

$$
\begin{aligned}
& \operatorname{Res}\left[F_{c_{i}^{(1)}}^{(A)}(z, \bar{z})\right]_{z=z_{+}}=\frac{Y_{+}}{p_{2}^{2}\left(z_{-}-z_{+}\right)^{3}} \ln \left(d_{i}\left(z_{+}, \bar{z}_{+}\right)\right) \\
& +\left.\frac{N\left(z_{+}, \bar{z}_{+}\right)}{p_{2}^{2}\left(z_{+}-z_{-}\right)^{2}} \frac{\partial \ln d_{i}(z, \bar{z})}{\partial z}\right|_{z=z_{+}}+\operatorname{Res}_{z=z_{+}}[R(z, \bar{z})],
\end{aligned}
$$

where

$$
Y_{+}=2 N\left(z_{+}, \bar{z}_{+}\right)+\left.\left(z_{-}-z_{+}\right) \frac{\partial N(z, \bar{z})}{\partial z}\right|_{z=z_{+}} .
$$

It is clear that after calculating residues of $F_{c_{i}^{(1)}}^{(A)}(z, \bar{z})$, logarithmic and rational functions of $z$ should vanish separately. Therefore, Eq. (8.69) implies that $Y_{+}=0$ and

$$
\sum_{z=z_{ \pm}}\left[\frac{N(z, \bar{z})}{p_{2}^{2}\left(z_{+}-z_{-}\right)^{2}} \frac{\partial \ln d_{i}(z, \bar{z})}{\partial z}+\operatorname{Res}[R(z, \bar{z})]\right]=0 .
$$

Eq. (8.71) is striking since it implies that neglecting logarithmic terms before computing the residues could, potentially, be problematic since logarithmic and rational functions mix. This is a consequence of the fact that higher-order poles in the $z$-complex plane appear in anti-derivatives of spurious terms. Mixing of rational and logarithmic functions is controlled by the $z$-derivatives of propagator $d_{i}$ evaluated at the position of the poles $z_{ \pm}$. Using Eqs. $(8.65,8.66)$ we find that

$$
\left.\frac{\partial^{n} d_{i}(z, \bar{z})}{\partial z^{n}}\right|_{z=z_{ \pm}, \bar{z}=\bar{z}_{ \pm}}=0,
$$

for $n \geq 1$. Hence, it follows from Eq. (8.71) that mixing of logarithmic and rational terms is, indeed, absent. This justifies Eq. (8.63) as a valid way to compute the double-cut reduction coefficient.

Before closing this Section, we point out that the method of computing the double-cut reduction coefficient [31], that is described in this Section, is applicable in a more general case, when arbitrary masses are allowed. Indeed, the original parametrization of the double-cut loop momentum in terms of polar and azimuthal angles Eq. (8.16) is valid independently of masses. The mass dependence appears in the absolute value of the transverse part of the doublecut momentum $l_{\perp}$, since the on-shell conditions change $l^{2}=m_{0}^{2},\left(q_{1}+l\right)^{2}=m_{1}^{2}$. Also, we note that when arbitrary masses are allowed, the coefficients $p_{1,4}$ in Eq. (8.66) receive equal shifts of their $l_{\perp}$-independent parts. However, because the derivation of Eq. (8.63) only depends on the combination $p_{1}-p_{4}$ and is valid for arbitrary $l_{\perp}$, we conclude that Eq. (8.63) allows the computation of the double-cut cut-constructible reduction coefficient in the most general case. We will comment on the applicability of this method to the computation of the rational part in the next Section. 


\subsection{Direct computation of the rational part}

In Section 8.1 we described how to compute the cut-constructible reduction coefficients directly using Forde's method [29]. Badger pointed out in Ref. [30] that this method can be further extended to compute the rational part. Below we explain how this can be done. We focus on the contribution of a massless scalar field to one-loop gluon scattering amplitudes. As follows from the supersymmetric decomposition of one-loop scattering amplitudes discussed in Ref. [73], the rational part of gluon amplitudes can be extracted if such contribution is known. We work in the four-dimensional helicity scheme. In this Section, we will use the notation $\mu^{2}=\left(l \cdot n_{\epsilon}\right)^{2}$.

We explained the parametrization of all unitarity cuts in Section 5, including their dependence on $\mu^{2}$. In what follows we start the discussion with the pentuple cut and then move to quadruple, triple and bubble cuts. We make maximal use of the discussion in Section 8.1 since, as we will see, we need only minimal modifications to obtain the rational part. We always assume that we cut a subset of $d_{0}, d_{1}, . ., d_{4}$ inverse propagators, as appropriate for a particular cut.

As discussed in Section 5.2, the parametrization of the pentuple cut is

$$
\tilde{e}_{01234}(l)=\tilde{e}_{01234}^{(0)} \mu^{2} .
$$

The power counting implies that the five-point master integral vanishes in the limit $D \rightarrow 4$,

$$
\int \frac{\mathrm{d}^{D} l}{(2 \pi)^{D}} \frac{\mu^{2}}{d_{0} d_{1} d_{2} d_{3} d_{4}} \rightarrow 0
$$

so that we do not need to compute the reduction coefficients $\tilde{e}_{01234}^{(0)}$.

Next, we consider the quadruple cut and choose the momentum parametrization to be

$$
l_{q, \pm}^{\nu}=V_{4}^{\nu} \pm l_{\perp} n_{4}^{\nu}+\mu n_{\epsilon}^{\nu} .
$$

As explained in Sect. 5, $V_{4}$ is a constant vector orthogonal to $n_{4}$ and $n_{\epsilon}$ and $l_{\perp}^{2}=-V_{4}^{2}-\mu^{2}$. Taking the quadruple cut we find

$$
\begin{aligned}
{\left[A_{1} A_{2} A_{3} A_{4}\right]\left(l_{q}\right) } & =\tilde{d}_{0123}^{(0)}+\tilde{d}_{0123}^{(1)}\left(l_{q} \cdot n_{4}\right)+\tilde{d}_{0123}^{(2)} \mu^{2} \\
& +\tilde{d}_{0123}^{(3)} \mu^{2}\left(l_{q} \cdot n_{4}\right)+\tilde{d}_{0123}^{(4)} \mu^{4}+\sum_{i} \frac{\tilde{e}_{0123 i}^{(0)} \mu^{2}}{d_{i}\left(l_{q}\right)} .
\end{aligned}
$$

By power counting it is easy to understand that only the term $\tilde{d}_{0123}^{(4)} \mu^{4}$ contributes to the rational part. Since the inverse Feynman propagator $d_{i}$ scales as $d_{i} \sim l_{\perp} \sim \mu$ in the limit of very large value of $\mu$, we conclude that performing Laurent expansion at $\mu=\infty$ and picking up the $\mu^{4}$ term gives the coefficient of the only master integral related to the quadruple cut contribution to the rational part. We find

$$
\tilde{d}_{0123}^{(4)}=\mathcal{L}_{\mu^{2}, 4}\left[A_{1} A_{2} A_{3} A_{4}\right]\left(l_{q, \pm}\right) .
$$


As a next step we discuss the triple cut. For definiteness, we assume that it corresponds to zeros of inverse propagators $d_{0}, d_{1}, d_{2}$. The momentum parametrization reads

$$
l_{t, \pm}^{\nu}=V_{3}^{\nu}+l_{\perp}\left(t n_{\mp}^{\nu}+t^{-1} n_{ \pm}^{\nu}\right)+\mu n_{\epsilon}^{\nu} .
$$

Similar to the case of the quadruple cut that we have already discussed, $V_{3}^{\mu}$ is a constant vector and $l_{\perp}^{2}=-V_{3}^{2}-\mu^{2}$. Taking the triple cut of the amplitude, we find

$$
\left[A_{1} A_{2} A_{3}\right]\left(l_{t}\right)=\tilde{c}_{012}\left(l_{t}\right)+\sum_{i} \frac{\tilde{d}_{012 i}\left(l_{t}\right)}{d_{i}\left(l_{t}\right)}+\sum_{i j} \frac{\tilde{e}_{012 i j}^{(0)} \mu^{2}}{d_{i}\left(l_{t}\right) d_{j}\left(l_{t}\right)},
$$

where $\tilde{c}_{012}\left(l_{t}\right)$ is given in Eq. (5.16). The rational part of the function $\tilde{c}_{012}$ reads

$$
\tilde{c}_{012}^{\mathrm{rat}}\left(l_{t}\right)=\tilde{c}_{012}^{(7)} \mu^{2}+\tilde{c}_{012}^{(8)}\left(l_{t} \cdot n_{3}\right) \mu^{2}+\tilde{c}_{012}^{(9)}\left(l_{t} \cdot n_{4}\right) \mu^{2},
$$

and $\tilde{c}_{012}^{(7)}$ is the relevant, non-evanescent reduction coefficient. To project onto $\tilde{c}_{012}^{(7)}$, we begin by performing Laurent expansion at $t=\infty$, as we did for the cut-constructible part. We find

$$
\mathcal{L}_{t, 0}\left[\tilde{c}_{012}\left(l_{t,+}\right)\right]=\tilde{c}_{012}^{(0)}+\tilde{c}_{012}^{(7)} \mu^{2}, \quad \mathcal{L}_{t, 0}\left[\frac{\tilde{e}_{012 i j}^{(0)} \mu^{2}}{d_{i}\left(l_{t,+}\right) d_{j}\left(l_{t,+}\right)}\right]=0,
$$

and

$$
\mathcal{L}_{t, 0}\left[\frac{\tilde{d}_{012 i}\left(l_{t,+}\right)}{d_{i}\left(l_{t,+}\right)}\right]=\left(\tilde{d}_{012 i}^{(1)}+\mu^{2} \tilde{d}_{012 i}^{(3)}\right) \frac{\tilde{n}_{4}^{(i)} \cdot n_{+}}{2 q_{i} \cdot n_{+}} .
$$

As we explained in Section 8.1, the contribution of the quadruple-cut coefficient is removed by taking the sum over two solutions $l_{t, \pm}$ Eq. (8.78) which, as follows from the parametrization, corresponds to taking the large- $t$ limit along both $n_{+}$ and $n_{-}$directions. Hence, we find

$$
\frac{1}{2} \sum_{i= \pm} \mathcal{L}_{t, 0}\left[A_{1} A_{2} A_{3}\right]\left(l_{i}\right)=\tilde{c}_{012}^{(0)}+\tilde{c}_{012}^{(7)} \mu^{2}
$$

The coefficient $\tilde{c}_{012}^{(7)}$ is then obtained by the application of an additional Laurent expansion operator, designed to pick up the $\mathcal{O}\left(\mu^{2}\right)$ term, to the triple cut

$$
\tilde{c}_{012}^{(7)}=\frac{1}{2} \sum_{i= \pm} \mathcal{L}_{\mu^{2}, 2}\left[\mathcal{L}_{t, 0}\left[A_{1} A_{2} A_{3}\left(l_{i}\right)\right]\right] .
$$

Finally, it is straightforward to extend this analysis to the double cut, following the discussion of the cut-constructible part. The parametrization of the momentum shown in Eq. (8.19) is extended in a straightforward way. We write

$$
l^{\mu}=-\frac{q_{1}^{\mu}}{2}+l_{\perp}\left(n_{2}^{\mu}(1-2 y)+z n_{-}^{\mu}+\frac{4 y(1-y)}{z} n_{+}^{\mu}\right)+\mu n_{\epsilon}^{\mu},
$$


where $l_{\perp}^{2}=-q_{1}^{2} / 4-\mu^{2}$. The function $\tilde{b}_{01}(l)$ gets an additional contribution $\tilde{b}_{01}^{(9)} \mu^{2}$ that leads to a rational part. Given the argument described in connection with the cut-constructible part, about the relation between $y$ and $\cos \theta$ integrations, it is clear that

$$
\mathcal{L}_{z, 0}\left[\mathcal{L}_{y, \geq 0}\left[\tilde{b}_{01}(z, y)\right]^{y^{m} \rightarrow f_{m}}\right]=\tilde{b}_{01}^{(0)}+\mu^{2} \tilde{b}_{01}^{(9)} .
$$

The final ingredients we need to compute the contribution of a double cut to the rational part, are the terms that come from the remnants of the triple- and other higher-multiplicity cuts. Similar to the cut-constructible part, we only look at the remnants of the triple cuts.

In line with what we did in the computation of the cut-constructible part, we apply the Laurent expansion procedure to the remnant of the triple cut. We can easily extend Eq. (8.24) to include $\mu^{2}$ by promoting all vectors and constants to $\mu^{2}$-dependent quantities. Then, because in the analysis of the cut-constructible part we never used the explicit form of $l_{\perp}$ and this is the only $\mu$-dependent quantity, that analysis is applicable also for the rational part. In particular, substitutions $z^{n} \rightarrow Z(n)$ that we derived in Sect. 8.1, remain unchanged. As a result, we find that Eq. (8.24) is valid, provided that the left-hand side is changed to $b_{01}^{(0)} \rightarrow b_{01}^{(0)}+b_{01}^{(9)} \mu^{2}$. Therefore, to find the reduction coefficient for the rational part of the two-point function, we only need to apply another Laurent expansion operator at $\mu=\infty$, to Eq. (8.24). We find

$$
\begin{aligned}
\tilde{b}_{01}^{(9)} & =\mathcal{L}_{\mu, 2}\left[\mathcal{L}_{z, 0}\left[\mathcal{L}_{y, \geq 0}\left[A_{1} A_{2}\right]^{y^{m} \rightarrow f_{m}}\right]\right] \\
& -\frac{1}{2} \sum_{i} \sum_{\alpha= \pm} \mathcal{L}_{\mu, 2}\left[\mathcal{L}_{z, \geq 0}\left[A_{1} A_{2} A_{3}\right]^{(i)}\left(z, y_{\alpha}^{(i)}\right)\right]^{z^{n} \rightarrow Z(n)}
\end{aligned}
$$

which is the formula derived in Ref. [30].

As our final comment, we point out that the procedure for calculating the double-cut cut-constructible coefficient suggested in Ref. [31] and explained in Section 8.2 can be easily generalized to deal with the rational part. Indeed, the double-cut loop momentum parametrization in terms of polar and azimuthal angles Eq. (8.16) is valid even if a $(D-4)$-dependent component of $l$ is added. Clearly, the on-shell condition gets modified and reads $l_{\perp}^{2}+\mu^{2}+q_{1}^{2} / 4=0$. While it does change $l_{\perp}$, it is easy to realize that the exact form of $l_{\perp}$ is irrelevant for the argument in Section 8.2. Hence, we conclude that if the $(D-4)$-dimensional part of the loop momentum is kept when the double cut of a one-loop scattering amplitude is computed, Eq. (8.63) generalizes in a sense that the left hand side becomes a rank-two polynomial of $\mu$

$$
\tilde{b}_{01}^{(0)}+\mu^{2} \tilde{b}_{01}^{(9)}=-\sum_{z \text { poles } \in D_{\infty}} \operatorname{Res}\left[F^{(A), \text { rat }}(z, \bar{z})\right] .
$$

The rational part of a double cut is then obtained by picking up the $\mu^{2}$ dependent term after computing the right hand side of Eq. (8.88). 


\subsection{Using the helicity formalism}

The method of generalized unitarity, as described above, provides an algorithm for computation of one-loop multi-leg amplitudes by extracting reduction coefficients of one-loop integrals. Since the algorithm is numerical, it can be easily implemented using the conventional relativistic formalism of Dirac spinors and polarization vectors. Tree amplitudes, required for the determination of the reduction coefficients, are computed numerically using recursion relations. This enables us to avoid dealing with analytic expressions for scattering amplitudes, making the method robust.

On the other hand, this procedure is amenable to significant simplifications if scattering amplitudes of massless particles are considered. In this case, analytic expressions for scattering amplitudes are often available, leading to deeper insights into the structure of gauge field theories. This is particularly true for amplitudes in non-abelian gauge field theories, where color-ordered $n$-gluon maximally helicity violating (MHV) tree amplitudes are given by a remarkably simple expression [110]

$$
m_{n}\left(1^{+}, 2^{+}, \ldots, i^{-}, \ldots, j^{-}, \ldots, n^{+}\right)=i \frac{\langle i j\rangle^{4}}{\langle 12\rangle\langle 23\rangle \ldots\langle n 1\rangle} .
$$

This result was conjectured as a generalization of analytic results obtained with helicity methods for four-, five- and six-gluon scattering amplitudes and later proven for an arbitrary number of gluons [28].

The spinor-helicity methods are also useful in loop computations [111]. However, because of the four-dimensional nature of spinor-helicity variables, these methods can not be used directly for computing the rational parts. With the advent of the unitarity methods simple analytic expressions could be derived for tree and loop amplitudes in QCD, in $N=4$ Super-Yang-Mills theory and in $N=8$ supergravity. Since a number of excellent reviews of spinor-helicity formalism are available in the literature $[82,83,112,113]$, we do not discuss it here. Nevertheless, since the helicity method is an important part of the toolkit of analytic computations in gauge field theories, we summarize the basics of the method in Appendix D. We also demonstrate in Sec. 9.6 how the spinor-helicity method can be used in conjunction with generalized unitarity by deriving compact results for the one-loop amplitude of the $q \bar{q} g g$ subprocess.

\section{Examples}

The goal of this Section is to discuss examples where the computation of the full answer, or some well-defined parts of it, can be performed with relative ease. We are particularly interested in cases where the peculiar nature of the rational part, and its relation to anomalous behavior of quantum field theories, becomes explicit. This feature is best illustrated by computing one-loop quantities that are finite but whose computation in four-dimensions - or better to say in unregularized quantum field theory - would have been impossible. In the literature, some calculations of the rational part of multi-gluon amplitudes are reported $[114,30]$; our examples below refer to a slightly different physics. 

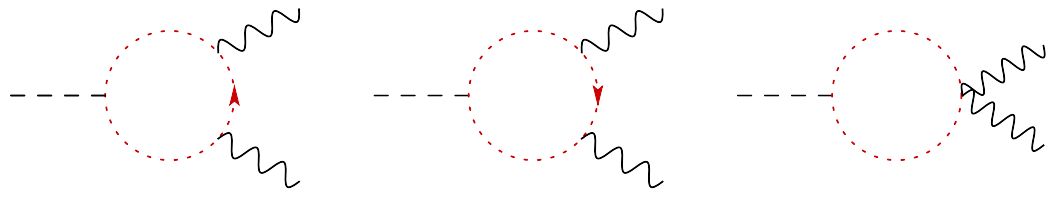

Figure 9.1: The three diagrams for the $H \rightarrow \gamma \gamma$ decay mediated by a loop of charged scalars.

\subsection{Higgs decay to two photons through massless scalars}

We begin by considering the decay of a scalar particle - we will call it the Higgs boson - to two massless gauge bosons - the photons - through a loop of massless charged scalars. We label those scalars as $\varphi^{ \pm}$. If physical justification is needed, the massless scalars can be thought of as longitudinal modes of $W$ bosons; treating longitudinal $W$-bosons as massless scalar particles in Higgs boson decays is justified by the Equivalence Theorem $[115,116]$ in the limit $m_{H} \gg m_{W}$. We will denote the coupling of the massless scalars to the Higgs boson as $g_{H}$, so that the $H \varphi^{2}$ vertex is $-i g_{H}$. We consider the decay of the Higgs boson with the mass $m_{H}$ in its rest frame. The photon momenta are taken to be $k_{1,2}=\left(m_{H} / 2,0,0, \pm m_{H} / 2\right)$. For the decay of the Higgs boson to occur, the photons should have equal helicities. Their polarization vectors are chosen to be

$$
e_{1,2}=\frac{1}{\sqrt{2}}(0,1, \pm i, 0)
$$

There are three different diagrams that contribute to the decay of the Higgs boson to two photons shown in Fig. 9.1. Given that virtual particles, as well as photons, are massless, the unitarity calculation includes two triple cuts and a double cut. The double cuts that have a single photon on one side of the cut can be disregarded because the corresponding master integrals are scaleless and therefore vanish.

We begin the computation with one of the triple cuts. The loop momentum must satisfy the cut constraints given by the following equations

$$
l^{2}=0, \quad\left(l+k_{1}\right)^{2}=0, \quad\left(l-k_{2}\right)^{2}=0 .
$$

These equations imply that $l$ must be orthogonal to $k_{1}$ and $k_{2},\left(l \cdot k_{1}\right)=\left(l \cdot k_{2}\right)=$ 0 , therefore the solution of these equations is simply given by $l^{\mu}=l_{\perp}^{\mu}+\left(l \cdot n_{\epsilon}\right) n_{\epsilon}$, where $l_{\perp} \cdot k_{1}=l_{\perp} \cdot k_{2}=0$ and $l_{\perp}^{2}+\left(l \cdot n_{\epsilon}\right)^{2}=0$. The numerator of the triple cut is given by the product of two $\varphi \varphi \gamma$ vertices and the $H \varphi^{2}$ vertex. Because $k_{1,2} \cdot e_{1,2}=0$, the numerator is proportional to $\left(l_{\perp} \cdot e_{1}\right)\left(l_{\perp} \cdot e_{2}\right)$. We can simplify this expression by using the specific form of the polarization vectors $e_{1,2}$. We write

$$
\begin{aligned}
& 4\left(l_{\perp} \cdot e_{1}\right)\left(l_{\perp} \cdot e_{2}\right)=2 l_{\perp, \mu} l_{\perp, \nu}\left(e_{1}^{\mu} e_{2}^{\nu}+e_{1}^{\nu} e_{2}^{\mu}\right) \\
& \quad=-2 l_{\perp}^{\mu} l_{\perp}^{\nu} \omega_{\mu \nu}\left(k_{1}, k_{2}\right)=2 l_{\perp}^{2}\left(e_{1} \cdot e_{2}\right)=-2\left(l \cdot n_{\epsilon}\right)^{2}\left(e_{1} \cdot e_{2}\right),
\end{aligned}
$$

where $\omega_{\mu \nu}$ is the projector on the full transverse space, c.f. Eq. (3.20). In Eq. (9.3) we used the fact that only its four-dimensional part $\left(e_{1}^{\mu} e_{2}^{\nu}+e_{1}^{\nu} e_{2}^{\mu}\right)$ 
contributes when contracted with $l_{\perp, \mu} l_{\perp, \nu}$. Hence, the numerator factor of the triple cut reads

$$
\mathcal{I}^{(3, a)}=-2 g_{H} e^{2}\left(l \cdot n_{\epsilon}\right)^{2}\left(e_{1} \cdot e_{2}\right) .
$$

Computing also the second triple cut, we obtain the contribution of the threepoint function to the amplitude

$$
\mathcal{A}_{t}=-2 g_{H} e^{2} \int \frac{\mathrm{d}^{D} l}{(2 \pi)^{D}}\left(\frac{\left(l \cdot n_{\epsilon}\right)^{2}\left(e_{1} \cdot e_{2}\right)}{l^{2}\left(l+k_{1}\right)^{2}\left(l-k_{2}\right)^{2}}+\frac{\left(l \cdot n_{\epsilon}\right)^{2}\left(e_{1} \cdot e_{2}\right)}{l^{2}\left(l+k_{2}\right)^{2}\left(l-k_{1}\right)^{2}}\right) .
$$

It is easy to compute the corresponding integrals since they do not depend on kinematics, c.f. Eq. (B.6),

$$
\int \frac{\mathrm{d}^{D} l}{(2 \pi)^{D}} \frac{\left(l \cdot n_{\epsilon}\right)^{2}}{d_{1} d_{2} d_{3}}=-\frac{i}{32 \pi^{2}}+O(\epsilon) .
$$

As the next step, we need to compute the double cut, with the Higgs boson on one side of the cut and both photons on the other. The momentum on the cut satisfies the constraint

$$
l^{2}=0, \quad(l-K)^{2}=0, \quad K=k_{1}+k_{2} .
$$

The momentum $l$ that satisfies these constraints is written as

$$
l^{\mu}=\frac{1}{2} K^{\mu}+l_{\perp}^{\mu}+\left(l \cdot n_{\epsilon}\right) n_{\epsilon}^{\mu},
$$

where $l_{\perp} \cdot K=l_{\perp} \cdot n_{\epsilon}=0$ and

$$
l_{\perp}^{2}+\left(l \cdot n_{\epsilon}\right)^{2}=-\frac{K^{2}}{4}=-\frac{m_{H}^{2}}{4} .
$$

The residue of the one-loop amplitude on the double cut is proportional to $\varphi(K-l)+\varphi(l) \rightarrow \gamma\left(k_{1}\right)+\gamma\left(k_{2}\right)$ on-shell scattering amplitude,

$$
A_{\varphi(K-l)+\varphi(l) \rightarrow \gamma\left(k_{1}\right)+\gamma\left(k_{2}\right)}^{\text {tree }}=-i e^{2}\left[\frac{\left(2 l \cdot e_{1}\right)\left(2 l \cdot e_{2}\right)}{\left(l+k_{1}\right)^{2}}+\frac{\left(2 l \cdot e_{2}\right)\left(2 l \cdot e_{1}\right)}{\left(l+k_{2}\right)^{2}}+2 e_{1} e_{2}\right],
$$

where we used the fact that $k_{1,2} \cdot e_{1}=k_{1,2} \cdot e_{2}=0$. Therefore the residue reads

$$
\mathcal{I}^{(2)}=-g_{H} e^{2}\left[4\left(l \cdot e_{1}\right)\left(l \cdot e_{2}\right)\left(\frac{1}{2 l \cdot k_{1}}+\frac{1}{2 l \cdot k_{2}}\right)+2 e_{1} \cdot e_{2}\right] .
$$

The first two terms can be simplified since

$$
2 l \cdot k_{1,2}=k_{1} \cdot k_{2}+2 l_{\perp} \cdot k_{1,2}=\frac{m_{H}^{2}}{2} \pm m_{H}\left(l \cdot n_{3}\right),
$$

where $n_{1}=(0,1,0,0), n_{2}=(0,0,1,0), n_{3}=(0,0,0,1)$. Moreover, using

$$
2\left(l \cdot e_{1}\right)\left(l \cdot e_{2}\right)=-e_{1} \cdot e_{2}\left[\left(l \cdot n_{1}\right)^{2}+\left(l \cdot n_{2}\right)^{2}\right]=e_{1} \cdot e_{2}\left[l_{\perp}^{2}+\left(l \cdot n_{3}\right)^{2}\right],
$$


we find

$$
\mathcal{I}^{(2)}=8 g_{H} e^{2} \frac{\left(l \cdot n_{\epsilon}\right)^{2} e_{1} \cdot e_{2}}{m_{H}^{2}-4\left(n_{3} \cdot l\right)^{2}} .
$$

We note that $\mathcal{I}^{(2)}$ in Eq. (9.14) is not yet a result for the double cut that can be easily translated into the reduction coefficient of the two-point function. For this last step, we need to subtract from Eq. (9.14) the contributions of all possible higher-point coefficients to the double cut. This procedure is explained in detail in Sect.5. In the present case we just need to subtract from Eq. (9.14) the contributions of the three-point functions in Eq. (9.5) to the double cut. Given the difference in the momentum parametrization in triple and double cuts, the term that needs to be subtracted reads

$$
\mathcal{I}_{\text {subtr }}=-\left.\frac{2 g_{H} e^{2} e_{1} \cdot e_{2}\left(l \cdot n_{\epsilon}\right)^{2}}{l^{2}}\right|_{l \rightarrow l_{1}}-\left.\frac{2 g_{H} e^{2} e_{1} \cdot e_{2}\left(l \cdot n_{\epsilon}\right)^{2}}{l^{2}}\right|_{l \rightarrow l_{2}},
$$

where $l_{1,2}=l-k_{1,2}$ and the momentum $l$ is given in Eq. (9.8). A simple computation yields

$$
\mathcal{I}_{\text {subtr }}=8 g_{H} e^{2} \frac{\left(l \cdot n_{\epsilon}\right)^{2} e_{1} \cdot e_{2}}{m_{H}^{2}-4\left(n_{3} \cdot l\right)^{2}} .
$$

Comparing Eq. (9.14) and Eq. (9.16), we see that complete double-cut contribution of the one-loop amplitude is contained in the triple cut which implies that the two-point function reduction coefficients vanish. Therefore, the oneloop amplitude for the Higgs boson decay to two photons through a massless scalar loop is given by Eq. (9.5) and is entirely due to the rational part of a three-point function. We obtain

$$
\mathcal{A}=i g_{H} \frac{\alpha}{2 \pi}\left(e_{1} \cdot e_{2}\right) .
$$

Finally, as we already mentioned, this calculation describes the $m_{H} \gg m_{W}$ limit of the $W$-boson loop contribution to $H \rightarrow \gamma \gamma$ scattering amplitude. The phenomenology of the Higgs boson decay into two photons was first discussed in Ref. [117].

\subsection{Higgs decay to two gluons through massive quarks loop}

In this Section, we discuss the decay of the Higgs boson to two gluons, through the triangle loop of massive quarks, see Fig. 9.2. Our goal here is to illustrate two different ways - the Passarino-Veltman reduction and the OPP procedure - to describe this process. We define the amplitude for Higgs boson decay to two gluons $H\left(k_{3}\right) \rightarrow g\left(k_{1}\right)+g\left(k_{2}\right)$ as

$$
\mathcal{M}_{h_{1}, h_{2}}=\delta_{a_{1}, a_{2}} \frac{g_{H} \alpha_{s}}{8 \pi} A, \quad A=\int \frac{d^{D} l}{i \pi^{D / 2}} \mathcal{A}_{h_{1}, h_{2}}\left(k_{1}, k_{2}, l\right),
$$

where $h_{1,2}$ and $a_{1,2}$ are the helicity and color labels of the two gluons, $g_{H}$ is the Yukawa coupling of the Higgs boson to massive fermions and $\alpha_{s}$ is the strong 

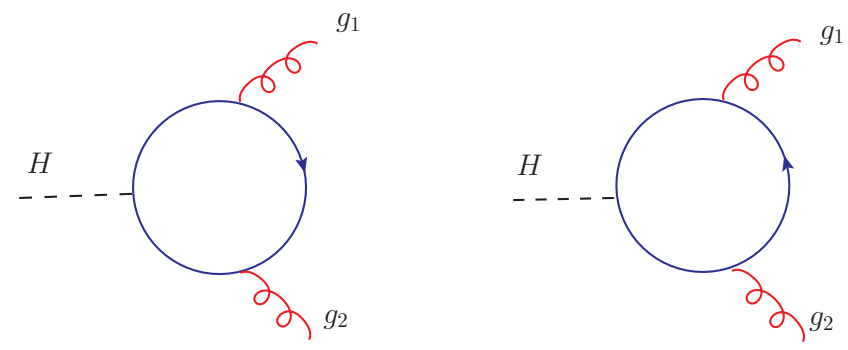

Figure 9.2: Diagrams that contribute to Higgs boson decay to two gluons through a massive quark loop.

coupling constant. Because the Higgs boson is a spinless particle, the two gluons in the final state must have identical helicities. Thanks to parity conservation, $M_{++}=M_{--}$, and we only need to consider the case $h_{1}=h_{2}=+1$. We work in the rest frame of the Higgs-boson, and choose the momenta and helicity vectors as

$$
\begin{aligned}
& k_{1,2}=\frac{m_{H}}{2}(1,0,0, \pm 1), \quad e_{1,2}^{+}=\frac{1}{\sqrt{2}}(0,1, \pm i, 0) \\
& k_{i} \cdot e_{1,2}^{+}=0, \quad e_{1}^{+} \cdot e_{2}^{+}=-1 .
\end{aligned}
$$

The integrand function can be written as

$$
\mathcal{A}=\frac{\operatorname{Tr}\left[(\hat{l}+m) \hat{e}_{1}\left(\hat{l}+\hat{k_{1}}+m\right) \hat{e}_{2}\left(\hat{l}+\hat{k}_{3}+m\right)\right]}{d_{0} d_{1} d_{3}}+[1 \leftrightarrow 2],
$$

where

$$
d_{0}=l^{2}-m^{2}, d_{1}=\left(l+k_{1}\right)^{2}-m^{2}, \quad d_{3}=\left(l+k_{3}\right)^{2}-m^{2} .
$$

Evaluating the trace and setting $\operatorname{Tr}[1]=2^{D / 2} \rightarrow 4$, we obtain

$$
\mathcal{A}=4 m\left\{\left[\frac{4\left(l \cdot e_{1}\right)\left(l \cdot e_{2}\right)+\frac{1}{2} m_{H}^{2}}{d_{0} d_{1} d_{3}}+\frac{1}{d_{0} d_{3}}\right]+[1 \leftrightarrow 2]\right\} .
$$

We consider the OPP reduction of the amplitude in Eq. (9.22). The OPP parametrization of the integrand reads

$$
\mathcal{A}=\frac{\tilde{c}_{1}(l)}{d_{0} d_{1} d_{3}}+\frac{\tilde{c}_{2}(l)}{d_{0} d_{2} d_{3}}+\frac{\tilde{b}_{03}(l)}{d_{0} d_{3}}+\ldots,
$$

where the points of ellipsis indicate contributions to the integrand that, when integrated over the loop momentum, can only depend on a single parameter - the quark mass. We begin by calculating the triple cut, specified by the condition 
$d_{0}=d_{1}=d_{3}=0$. The loop momentum on this triple cut is parametrized similar to Eq. (8.3)

$$
l_{1}^{\mu}=-k_{1}^{\mu}+i \frac{l_{\perp}}{\sqrt{2}}\left(z e_{1}^{\mu}+\frac{1}{z} e_{2}^{\mu}\right)+\mu n_{\epsilon}^{\mu}, \quad l_{\perp}^{2}=m^{2}-\mu^{2},
$$

where $\mu=l \cdot n_{\epsilon}$. Using the cut momentum Eq. (9.24) in Eq. (9.20) to compute the relevant residue, we obtain

$$
\tilde{c}_{1}\left(z, \mu^{2}\right)=\lim _{d_{0,1,3} \rightarrow 0} d_{0} d_{1} d_{3} \mathcal{A}=4 m\left[\frac{1}{2} m_{H}^{2}-2 m^{2}+2 \mu^{2}\right] .
$$

The generic parametrization of the triple cut is given in Eq. (8.8); it reads

$$
\tilde{c}_{1}\left(z, \mu^{2}\right)=\sum_{j=-3}^{3} \tilde{c}_{1}^{(j)} z^{j}+\tilde{c}_{1}^{(\epsilon)}(z) \mu^{2} .
$$

Comparing Eqs. (9.25) and (9.26), we find

$$
\tilde{c}_{1}^{(0)}=4 m\left[\frac{1}{2} m_{H}^{2}-2 m^{2}\right], \quad \tilde{c}_{1}^{(\epsilon)}(z)=8 m, \quad \tilde{c}_{1}^{(j)}=0, j \neq 0 .
$$

The coefficient of the second triple cut is obtained by the exchange of the indices $1 \leftrightarrow 2$; we conclude that $c_{2}=c_{1}$.

Next, we need to evaluate contributions from double cuts. We begin by considering the cut $d_{0}(l)=0, d_{3}(l)=0$. The loop momentum is parametrized as

$$
l_{03}^{\mu}=-\frac{k_{3}^{\mu}}{2}+i l_{\perp}\left(\frac{k_{12}^{\mu}}{m_{H}}(1-2 y)+z \frac{e_{1}^{\mu}}{\sqrt{2}}+4 y(1-y) \frac{e_{2}^{\mu}}{z \sqrt{2}}\right)+\mu n_{\epsilon}^{\mu},
$$

where $k_{12}=k_{1}-k_{2}$ and $l_{\perp}^{2}=m^{2}-m_{H}^{2} / 4-\mu^{2}$.

We can compute the double-cut using this momentum parametrization in the trace, Eq. (9.22) and subtracting from it the double-cut remnant of a triple cut contribution Eq. (9.25)

$$
\begin{aligned}
& \tilde{b}_{03}(l)=\lim _{d_{0,3} \rightarrow 0} d_{0} d_{3}\left[\mathcal{A}-\left(\frac{\tilde{c}_{1}(l)}{d_{0} d_{1} d_{3}}+\frac{\tilde{c}_{2}(l)}{d_{0} d_{2} d_{3}}\right)\right] \\
& =\left[\left(4\left(l_{03} \cdot e_{1}\right)\left(l_{03} \cdot e_{2}\right)+2 m^{2}-2 \mu^{2}\right)\left(\frac{1}{d_{1}}+\frac{1}{d_{2}}\right)+2\right] .
\end{aligned}
$$

It is easy to see that the following relations are true

$$
\begin{aligned}
& 4\left(l_{03} \cdot e_{1}\right)\left(l_{03} \cdot e_{2}\right)=-2 l_{\perp}^{2}\left[1-(1-2 y)^{2}\right], \\
& d_{1} d_{2}=m_{H}^{2}\left[\left(m^{2}-\mu^{2}\right)(1-2 y)^{2}+\frac{m_{H}^{2}}{4}\left[1-(1-2 y)^{2}\right]\right],
\end{aligned}
$$

and $d_{1}+d_{2}=-m_{H}^{2}$. Inserting these expressions into Eq. (9.29) we find that $\tilde{b}_{03}(l)=0$. 
We can now argue that the remaining double- and single-cut contributions should vanish as well. Indeed, it is easy to see that all these contributions can be written through a single divergent vacuum bubble master integral $I_{1}\left(\mathrm{~m}^{2}\right)$, cf. Eq. (2.6). Since this integral is ultraviolet-divergent, and the amplitude $H \rightarrow g g$ is ultraviolet-finite, the reduction coefficient of this reduction integral must vanish. We conclude that the $H \rightarrow g g$ amplitude is completely determined by the triple cut contribution. To arrive at the final answer, we need to use the triple cut reduction coefficient, in conjunction with the values of master integrals. We find

$$
A=2 \tilde{c}_{1}^{(0)} I_{3}[1]+2 \tilde{c}_{1}^{(\epsilon)} I_{3}\left[\mu^{2}\right], \quad I_{3}\left[x^{2}\right]=\int \frac{\mathrm{d}^{D} l}{i \pi^{D / 2}} \frac{x^{2}}{d_{0} d_{1} d_{3}},
$$

where $I_{3}[1]$ denotes the standard scalar triangle integral and $I_{3}\left[\mu^{2}\right]$ can be obtained from Eq. (B.6). We find

$$
I_{3}[1]=I_{3}\left(0,0, m_{H}^{2}, m^{2}, m^{2}, m^{2}\right)=\frac{2}{m_{H}^{2}} f(\tau), \quad I_{3}\left[\mu^{2}\right]=-\frac{1}{2},
$$

where

$$
f(\tau)= \begin{cases}-\arcsin ^{2} \frac{1}{\sqrt{\tau}} & , \quad \text { if } \tau>1, \\ +\frac{1}{4}\left(\ln \frac{1+\sqrt{1-\tau}}{1-\sqrt{1-\tau}}-i \pi\right)^{2}, & \text { if } \tau \leq 1 .\end{cases}
$$

Putting everything together, we obtain the well-known result (see e.g. Ref. [118]),

$$
A=8 m[(1-\tau) f(\tau)-1], \quad \tau=\frac{4 m^{2}}{m_{H}^{2}} .
$$

We now briefly discuss how to derive the same result using the PassarinoVeltman reduction. We use the results for the Passarino-Veltman reduction given in Section 2. The integral of the rank two tensor part of the first term in Eq. (9.22) is given by the $C_{00}$ function of Eq. (2.12). We note that

$$
\left(l \cdot e_{1}\right)\left(l \cdot e_{2}\right)=-\frac{1}{2} w^{\mu \nu} l_{\mu} l_{\nu}, \quad w^{\mu \nu}=-e_{1}^{\mu} e_{2}^{\nu}-e_{1}^{\mu} e_{2}^{\nu}, \quad w_{\mu}^{\mu}=2,
$$

and, therefore,

$$
\int \frac{d^{D} l}{i \pi^{D / 2}} \frac{4\left(l \cdot e_{1}\right)\left(l \cdot e_{2}\right)}{d_{0} d_{1} d_{3}}=-4 C_{00}(1,2,3) .
$$

Using the reduction equations $(2.13,2.14,2.20,2.26)$ we obtain

$$
\begin{aligned}
-4 C_{00}(1,2,3)= & -\frac{2}{D-2}\left(2 m^{2} C_{0}(1,2,3)+B_{0}(1,3)\right)= \\
& -2 m^{2} I_{3}[1]-I_{2}\left(m_{H}^{2}, m^{2}, m^{2}\right)-1 .
\end{aligned}
$$

By inserting this value of the tensor integral into the expression of the amplitude $\mathcal{A}$ of Eq. (9.22), the scalar bubble integral function $I_{2}\left(m_{H}^{2}, m^{2}, m^{2}\right)$ is cancelled 


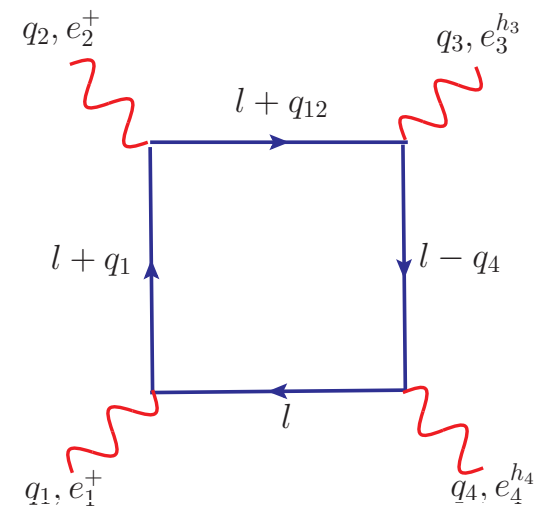

Figure 9.3: One of the diagrams that contributes to photon-photon scattering.

by the contribution from the two-denominator term in Eq. (9.22) and we reproduce the previous result (9.34). As already has been pointed out in Section 2 in the Passarino-Veltman reduction the rational part is obtained by a cancellation of the $1 / \epsilon$ singularity of the two-point scalar integral with the $\epsilon$-dependent coefficient $2 /(D-2)$, that appears in the reduction formula, Eq. (9.37).

\subsection{The rational part of the four-photon scattering amplitudes}

Our next example concerns the photon-photon scattering through a loop of massive fermions. This process is described by an ultraviolet-finite scattering amplitude that, however, can not be computed in four dimensions. As a consequence, the four-photon scattering amplitude has a non-trivial rational part whose computation we now describe.

We denote the incoming momentum of the $i$-th photon by $q_{i}$ and its polarization vector by $e_{i}, i=1, \ldots, 4$. There are six diagrams that contribute to the scattering amplitude, but only three of them are independent. This allows us to write

$$
\mathcal{M}_{\text {tot }}=-2 i \alpha^{2} \mathcal{M}, \quad \mathcal{M}=\sum_{i=a, b, c} \mathcal{M}_{i}
$$

Contribution of the diagram $a$, shown in Fig. 9.3, reads

$$
\mathcal{M}_{a}=-i \int \frac{\mathrm{d}^{D} l}{\pi^{D / 2}} \frac{\operatorname{Num}_{a}\left(l, m,\left\{q_{i}\right\},\left\{e_{i}\right\}\right)}{d_{0} d_{1} d_{2} d_{3}},
$$

where $d_{0}=\left(l^{2}-m^{2}\right), d_{1}=\left(l+q_{1}\right)^{2}-m^{2}, d_{2}=\left(l+q_{12}\right)^{2}-m^{2}, d_{3}=\left(l-q_{4}\right)^{2}-m^{2}$, $q_{12}=q_{1}+q_{2}$ and the numerator function is

$$
\operatorname{Num}_{a}=\operatorname{Tr}\left(\hat{e}_{1}(\hat{l}+m) \hat{e}_{4}\left(\hat{l}-\hat{q}_{4}+m\right) \hat{e}_{3}\left(\hat{l}+\hat{q}_{12}+m\right) \hat{e}_{2}\left(\hat{l}+q_{1}+m\right)\right) .
$$

Contributions of diagrams $b, c$ are obtained from Eq. (9.39) by simple substitutions, $\mathcal{M}_{b}=\mathcal{M}_{a}(3 \leftrightarrow 4)$ and $\mathcal{M}_{c}=\mathcal{M}_{a}(2 \leftrightarrow 3)$. 
The polarization vector $e$ of a massless gauge boson can be chosen in such a way that it satisfies a constraint $e \cdot n_{\text {aux }}$, where $n_{\text {aux }}$ is an auxiliary light-like vector. We use this freedom and choose $q_{2}$ to be an auxiliary vector for $e_{1}, q_{1}$ for $e_{2}, q_{3}$ for $e_{4}$ and $q_{4}$ for $e_{3}$. We shall refer to these choices as gauge fixing conditions. As an example, we consider positive helicities of the photons 1 and 2. Then we can write

$$
e_{1}^{+\mu} e_{2}^{+\nu}=\frac{1}{2}\left(e_{1}^{+} \cdot e_{2}^{+}\right)\left(\omega^{\mu \nu}\left(q_{1}, q_{2}\right)-i\left(q_{1} \cdot q_{2}\right)^{-1} \epsilon^{\mu \nu \alpha \beta} q_{1, \alpha} q_{2, \beta}\right),
$$

where $\omega^{\mu \nu}\left(q_{1}, q_{2}\right)$ is the metric tensor of the linear vector space that is transverse to the two light-like vectors $q_{1}$ and $q_{2}$

$$
\omega^{\mu \nu}\left(q_{1}, q_{2}\right)=g_{4}^{\mu \nu}-\frac{q_{1}^{\mu} q_{2}^{\nu}+q_{1}^{\nu} q_{2}^{\mu}}{q_{1} \cdot q_{2}} .
$$

We note that $g_{4}^{\mu \nu}$ is the metric tensor of the four-dimensional space.

As follows from Eqs. $(9.39,9.40)$, the photon-photon scattering is described by the four-point tensor integrals up to rank four. As we explained in Sect. 5.3, only rank-four and rank-three four-point tensor integrals contain a rational part; for this reason we focus on those integrals in what follows. We begin by considering the rational part of the photon scattering amplitude that originates from the rank-four tensor integrals. We shall use the notation $\mathcal{R}[\mathcal{O}]$, to denote the rational part of the amplitude $\mathcal{O}$. For diagram $a$ the corresponding expression reads

$$
\mathcal{R}\left[\mathcal{M}_{a}^{(4)}\right]=\operatorname{Tr}\left[\hat{e}_{1} \gamma^{\mu} \hat{e}_{4} \gamma^{\nu} \hat{e}_{3} \gamma^{\alpha} \hat{e}_{2} \gamma^{\beta}\right] \mathcal{R}_{\mu \nu \alpha \beta}^{4, a},
$$

where

$$
\mathcal{R}_{\mu \nu \alpha \beta}^{4, a}=-i \mathcal{R}\left[\int \frac{\mathrm{d}^{D} l}{\pi^{D / 2}} \times \frac{l_{\mu} l_{\nu} l_{\alpha} l_{\beta}}{d_{0} d_{1} d_{2} d_{3}}\right] .
$$

We can calculate trace in Eq. (9.43) and simplify it by noticing that the rank-four tensor integral is fully-symmetric and that, when all of its indices are contracted, the rational part vanishes. This allows us to write

$$
\mathcal{R}\left[\mathcal{M}_{a}^{(4)}\right]=\left(32 e_{1}^{\mu} e_{2}^{\nu} e_{3}^{\alpha} e_{4}^{\beta}-8 f_{1,4 ; 2,3}^{\mu \nu} g^{\alpha \beta}-8 f_{1,2 ; 3,4}^{\mu \nu} g^{\alpha \beta}\right) R_{\mu \nu \alpha \beta}^{4, a},
$$

where $f_{i j ; k m}^{\mu \nu}=e_{i}^{\mu} e_{j}^{\nu}\left(e_{k} \cdot e_{m}\right)+e_{k}^{\mu} e_{m}^{\nu}\left(e_{i} \cdot e_{j}\right)$. A computation of a similar contribution for other diagrams amounts to a simple permutation of momenta and polarization vectors. The complete result for the rational part that originates from the rank-four tensor integrals reads

$$
\begin{aligned}
& \mathcal{R}\left[\mathcal{M}^{(4)}\right]=32 e_{1}^{\mu} e_{2}^{\nu} e_{3}^{\alpha} e_{4}^{\beta} R_{\mu \nu \alpha \beta}^{4}-8 f_{1,4 ; 2,3}^{\mu \nu} g^{\alpha \beta}\left(R_{\mu \nu \alpha \beta}^{4, a}+R_{\mu \nu \alpha \beta}^{4, c}\right) \\
& -8 f_{1,2 ; 3,4}^{\mu \nu} g^{\alpha \beta}\left(R_{\mu \nu \alpha \beta}^{4, a}+R_{\mu \nu \alpha \beta}^{4, b}\right)-8 f_{1,3 ; 2,4}^{\mu \nu} g^{\alpha \beta}\left(R_{\mu \nu \alpha \beta}^{4, b}+R_{\mu \nu \alpha \beta}^{4, c}\right),
\end{aligned}
$$

where $R_{\mu \nu \alpha \beta}^{4}=\sum_{i=a, b, c} R_{\mu \nu \alpha \beta}^{4, i}$. 
All but one term in Eq. (9.46) include a contraction of the metric tensor $g^{\alpha \beta}$ with the rational part of various rank-four tensors. Those terms are easy to compute because such a contraction gives a rank-two four-point function which does not have a rational part and a rank-two three-point function whose rational part is simple, cf. Appendix B. We obtain

$$
\begin{aligned}
& g^{\alpha \beta} R_{\mu \nu \alpha \beta}^{4, a}=g^{\alpha \beta} R_{\mu \nu \alpha \beta}^{4, c}=\frac{1}{4} \omega_{\mu \nu}\left(q_{2}, q_{3}\right), \\
& g_{\alpha \beta} R_{\mu \nu \alpha \beta}^{4, b}=\frac{1}{4} \omega_{\mu \nu}\left(q_{2}, q_{4}\right) .
\end{aligned}
$$

Hence, the rational part of the amplitude related to the rank-four tensor contribution is

$$
\begin{gathered}
\mathcal{R}\left[\mathcal{M}^{(4)}\right]=32 e_{1}^{\mu} e_{2}^{\nu} e_{3}^{\alpha} e_{4}^{\beta} R_{\mu \nu \alpha \beta}^{4}-4 f_{1,4 ; 2,3}^{\mu \nu} \omega_{\mu \nu}\left(q_{2}, q_{3}\right) \\
-2\left(f_{1,2 ; 3,4}^{\mu \nu}+f_{1,3 ; 2,4}^{\mu \nu}\right)\left(\omega_{\mu \nu}\left(q_{2}, q_{3}\right)+\omega_{\mu \nu}\left(q_{2}, q_{4}\right)\right) .
\end{gathered}
$$

As the next step, we consider the rank-three part of the amplitude. There are more terms that contribute in this case but a simplification comes from the observation that if any pair of indices of a rank-three four-point function is contracted with the metric tensor, the resulting integrals have no rational part. We compute traces, use the gauge fixing conditions for the polarization vectors and disregard all terms where two indices of the rank three tensor are contracted. For the diagram $a$ we find

$$
\begin{aligned}
\mathcal{R}\left[\mathcal{M}_{a}^{(3)}\right] & =-16\left(\left(e_{1} \cdot e_{2}\right) e_{4}^{\mu} e_{3}^{\nu} q_{1}^{\alpha}+\left(e_{2} \cdot e_{3}\right) e_{4}^{\mu} e_{1}^{\nu} q_{12}^{\alpha}\right. \\
& \left.-\left(e_{4} \cdot e_{3}\right) e_{1}^{\mu} e_{2}^{\nu} q_{4}^{\alpha}\right) R_{\mu \nu \alpha}^{3, a}\left(q_{1}, q_{12},-q_{4}\right),
\end{aligned}
$$

where the momenta of three propagators that enter the rank-three box integral are shown explicitly. We can simplify this expression using the symmetry of $R_{3, a}^{\mu \nu \alpha}$ with respect to its Lorentz indices and the fact that in Eq. (9.49) $R_{3, a}^{\mu \nu \alpha}$ is always contracted with one of the propagator momenta, projecting the general expression for the rational part of the rank-three four-point functions, that is found in Appendix B, on one of the vectors in the van Neerven-Vermaseren basis. Moreover, for simplification purposes, it is useful to write the rational part of the rank-three four-point integral as

$$
\begin{aligned}
& R_{3, a}^{\mu \nu \alpha}\left(q_{1}, q_{12},-q_{4}\right)=\frac{1}{8}\left(v_{1}^{\alpha}\left(\omega^{\mu \nu}\left(q_{3}, q_{4}\right)-\omega^{\mu \nu}\left(q_{2}, q_{3}\right)\right)\right. \\
& \left.+v_{2}^{\alpha}\left(\omega^{\mu \nu}\left(q_{1}, q_{4}\right)-\omega^{\mu \nu}\left(q_{2}, q_{3}\right)\right)+v_{3}^{\alpha}\left(\omega^{\mu \nu}\left(q_{1}, q_{2}\right)-\omega^{\mu \nu}\left(q_{2}, q_{3}\right)\right)\right),
\end{aligned}
$$

where the vectors $v_{1,2,3}$ are such that the only non-vanishing scalar products are $v_{1} \cdot q_{1}=1, v_{2} \cdot q_{12}=1$ and $v_{3} \cdot q_{4}=-1$. We use Eq. (9.50) in Eq. (9.49), obtain contributions of other diagrams by permutations of momenta and polarization vectors, and arrive at the following result for the contribution of rank-three 
tensors to the rational part

$$
\begin{aligned}
& \mathcal{R}\left[\mathcal{M}^{(3)}\right]=-4 \sum_{i \neq j \neq k \neq m}\left(e_{i} \wedge e_{j}\right)_{i j}\left(e_{k} \cdot e_{m}\right)+4 f_{1,4 ; 2,3}^{\mu \nu} \omega_{\mu \nu}\left(q_{2}, q_{3}\right) \\
& +2\left(f_{1,2 ; 3,4}^{\mu \nu}+f_{1,3 ; 2,4}^{\mu \nu}\right)\left(\omega^{\mu \nu}\left(q_{2}, q_{3}\right)+\omega^{\mu \nu}\left(q_{2}, q_{4}\right)\right) \\
& +32\left(e_{1}^{\mu} e_{2}^{\nu} e_{4}^{\alpha}\left(e_{3} \cdot q_{1}\right)+e_{1}^{\mu} e_{3}^{\nu} e_{4}^{\alpha}\left(e_{2} \cdot q_{3}\right)\right) R_{3, c}^{\mu \nu \alpha}\left(q_{1}, q_{13},-q_{4}\right) .
\end{aligned}
$$

In Eq. (9.51), we introduced the notation

$$
\left(e_{i} \wedge e_{j}\right)_{i j}=e_{i}^{\mu} \omega_{\mu \nu}\left(q_{i}, q_{j}\right) e_{j}^{\nu}
$$

The term with $R_{3, c}$ in Eq. (9.51) does not contribute. We can see this using the explicit expression

$$
\begin{aligned}
& R_{3, c}^{\mu \nu \alpha}\left(q_{1}, q_{13},-q_{4}\right)=\frac{1}{8}\left(v_{1}^{\alpha}\left(\omega^{\mu \nu}\left(q_{2}, q_{4}\right)-\omega^{\mu \nu}\left(q_{2}, q_{3}\right)\right)\right. \\
& \left.+v_{2}^{\alpha}\left(\omega^{\mu \nu}\left(q_{1}, q_{4}\right)-\omega^{\mu \nu}\left(q_{2}, q_{3}\right)\right)+v_{3}^{\alpha}\left(\omega^{\mu \nu}\left(q_{1}, q_{3}\right)-\omega^{\mu \nu}\left(q_{2}, q_{3}\right)\right)\right),
\end{aligned}
$$

so that

$$
e_{1}^{\nu} e_{2}^{\alpha} R_{3, c}^{\mu \nu \alpha}=e_{3}^{\nu} e_{4}^{\alpha} R_{3, c}^{\mu \nu \alpha}=0
$$

thanks to our gauge fixing conditions. Finally, if we combine the rank-four and the rank-three parts of the amplitude, terms with $f_{i j ; k m}^{\mu \nu}$ cancel out and we obtain the simple result for the full rational part of the four-photon scattering amplitude

$$
\mathcal{R}[\mathcal{M}]=32 e_{1}^{\mu} e_{2}^{\nu} e_{3}^{\alpha} e_{4}^{\beta} R_{\mu \nu \alpha \beta}^{4}-4 \sum_{i \neq j \neq k \neq m}\left(e_{i} \wedge e_{j}\right)_{i j}\left(e_{k} \cdot e_{m}\right)
$$

We are left with the question of how to compute the first term in Eq. (9.55), i.e. the rational part of the rank-four box contracted with the polarization vectors. To do this, we use Eq. (9.41) where the tensor product of two photon polarization vectors is written as a linear combination of the metric tensor and the Levi-Civita tensor and observe that the Levi-Civita tensor does not contribute because of the symmetry of $R_{4}^{\mu \nu \alpha \beta}$. We obtain

$$
\begin{aligned}
& 32 e_{1, \mu}^{+} e_{2, \nu}^{+} R_{4}^{\mu \nu \alpha \beta}=16\left(e_{1}^{+} \cdot e_{2}^{+}\right) \omega^{\mu \nu}\left(q_{1}, q_{2}\right) R_{4}^{\mu \nu \alpha \beta} \\
& =16\left(e_{1}^{+} \cdot e_{2}^{+}\right)\left(g_{4}^{\mu \nu}-4 \frac{q_{1}^{\mu} q_{2}^{\nu}}{q_{12}^{2}}\right) R_{\mu \nu \alpha \beta}^{4} \\
& =16\left(e_{1}^{+} \cdot e_{2}^{+}\right)\left(g^{\mu \nu}-g_{-2 \epsilon}^{\mu \nu}-4 \frac{q_{1}^{\mu} q_{2}^{\nu}}{q_{12}^{2}}\right) R_{\mu \nu \alpha \beta}^{4} .
\end{aligned}
$$

Note that in the last step in Eq. (9.56), we wrote $g_{4}^{\mu \nu}$ - the metric tensor of the four-dimensional space - as a difference of the full metric tensor $g^{\mu \nu}$ and the metric tensor of the $(D-4)$-dimensional space. The contraction of $R_{4}^{\mu \nu \alpha \beta}$ with the tensor $g_{\mu \nu}$ is straightforward to compute since the resulting integral is 
just the rank-two three point function whose rational part is very simple. To calculate $q_{1}^{\mu} q_{2}^{\nu} R_{\mu \nu \alpha \beta}^{4}$, we contract the momentum $q_{1}$ with the loop momentum $l$, rewrite the scalar product through the inverse propagators and shift the loop momentum, as appropriate, to ensure cancellations of (parts) of diagrams. We obtain

$$
2 q_{1}^{\mu} R_{\mu \nu \alpha \beta}^{4}=\frac{1}{4}\left(q_{34}^{\{\nu} \omega^{\alpha \beta\}}\left(q_{3}, q_{4}\right)+q_{14}^{\{\nu} \omega^{\alpha \beta\}}\left(q_{2}, q_{3}\right)-q_{3}^{\{\nu} \omega^{\alpha \beta\}}\left(q_{2}, q_{4}\right)\right),
$$

where $q_{14}=q_{1}-q_{4}$. Eq. (9.57) - a remnant of the gauge-invariance - allows us to get rid of all rank four-boxes and rank-three triangles whose rational parts are complicated. The last thing we need is the contraction of $R_{4}^{\mu \nu \alpha \beta}$ with $g_{-2 \epsilon}^{\mu \nu}$. We obtain

$$
g_{-2 \epsilon}^{\mu \nu} R_{\mu \nu \alpha \beta}=\frac{1}{24 \epsilon}\left(-2 \epsilon g_{\alpha \beta}+2 g_{\alpha \beta}^{-2 \epsilon}\right) .
$$

Putting the three terms together, we find

$$
32 e_{1}^{+, \mu} e_{2}^{+, \nu} e_{3}^{\alpha} e_{4}^{\beta} R_{\mu \nu \alpha \beta}^{4}=12\left(e_{1}^{+} \cdot e_{2}^{+}\right)\left(e_{3} \cdot e_{4}\right) .
$$

Hence, the rational part of the full photon-photon scattering amplitude reads

$$
\mathcal{R}[\mathcal{M}]=12\left(e_{1}^{+} \cdot e_{2}^{+}\right)\left(e_{3} \cdot e_{4}\right)-4 \sum_{i \neq j \neq k \neq m}\left(e_{i} \wedge e_{j}\right)\left(e_{k} \cdot e_{m}\right) .
$$

We can further simplify the sum in the above equation using the gauge fixing condition. We find

$$
\mathcal{R}\left[\mathcal{M}^{++\lambda_{3} \lambda_{4}}\right]=8 i \alpha^{2}\left(e_{1}^{+} e_{2}^{+}\right)\left[1+\left(1-\bar{\lambda}_{34}\right) \frac{\left(q_{1} \cdot q_{2}\right)\left(q_{1} \cdot e_{3}\right)\left(q_{1} \cdot e_{4}\right)}{q_{1} \cdot q_{3} q_{1} \cdot q_{4}}\right],
$$

where $\bar{\lambda}_{34}=\left(\lambda_{3}+\lambda_{4}\right) / 2$ is the average helicity of the photons 3 and 4 .

We note that the result for the rational part of the photon-photon scattering amplitude Eq. (9.61) agrees with the explicit computation in Ref. [119] which was performed for massless virtual fermions. Our derivation shows that the rational part of the four-photon scattering amplitude is, in fact, mass-independent. In particular, the rational part is given by Eq. (9.61) even if the energy of the photon-photon scattering is much smaller than the fermion mass. We emphasize that this feature is striking because the full amplitude in this limit does depend on the mass of the virtual fermion. In fact, it is well known that the full amplitude for photon-photon scattering, given by the sum of the cut-constructible and the rational parts, scales as

$$
\mathcal{M} \sim e^{4} \frac{s^{2}}{m^{4}}, \quad m \gg \sqrt{s},
$$

where $\sqrt{s}$ is the energy of the photon-photon collision. It follows that in order to make Eqs. $(9.61,9.62)$ consistent, we must assume that the rational part Eq. (9.61) cancels the mass-independent term in the cut-constructible part, making the full amplitude consistent with the required mass scaling. However, such cancellations signal potential sources of numerical instabilities at low energies, $\sqrt{s} \ll m$, which may be hard to deal with. 


\subsection{Rational parts of $n$-photon scattering amplitudes, $n>4$}

We comment on the rational part of higher-multiplicity photon scattering amplitudes. For six-photon scattering, the rational part was computed in Ref. [119] and was found to be zero

$$
\mathcal{R}\left[\mathcal{M}_{6 \gamma}\right]=0 .
$$

Later, it was argued in Ref. [32] that rational parts of $n$-photon scattering amplitudes vanish for $n \geq 6$. In what follows, we summarize arguments given in Ref. [32]. In the remainder of this Section, we consider photon scattering mediated by massless fermions.

To simplify the problem, it is useful to invoke the supersymmetric decomposition of scattering amplitudes [73]. This decomposition allows us to argue that rational parts of a photon scattering amplitude mediated by fermions are identical to the rational parts of a photon scattering amplitude mediated by loops of charged scalars $\varphi$. Cuts of those one-loop amplitudes lead to tree amplitudes that describe $0 \rightarrow 2 \varphi+n \gamma$ scattering. We first discuss properties of tree scattering amplitudes. Once these amplitudes are understood, it is straightforward to present arguments that explain the vanishing of the rational parts for multi-photon scattering amplitudes.

We take $p_{a, b}$ as momenta of the two scalars and denote the momenta of the photons as $k_{i}, i=1, . ., n$. In the spirit of BCFW momentum deformation [104], we shift momenta $p_{a, b}$ by the light-like momentum $q$ that is orthogonal to $p_{a, b}$. We then write

$$
p_{a, b} \rightarrow p_{a, b}(z)=p_{a, b} \pm z q .
$$

We are interested in the behavior of $0 \rightarrow 2 \varphi+n \gamma$ scattering amplitudes in the limit $z \rightarrow \infty$. To simplify studies of the large- $z$ limit, we choose vector $q$ as auxiliary quantization vector for all photons, so that $e_{i} \cdot q=0, i=1, . . n$. Since the $\varphi^{2} \gamma$ vertex is proportional to the difference ${ }^{15}$ of the momenta of the two scalars, choosing $e_{i} \cdot q=0$ ensures that the triple vertex becomes $z$-independent. The $\varphi^{2} \gamma \gamma$ vertex, on the other hand, is $z$-independent in any gauge and all the off-shell propagators in the scattering amplitudes scale as $\mathcal{O}\left(z^{-1}\right)$ in the large- $z$ limit. Hence, the large $z$-behavior of $0 \rightarrow 2 \varphi+n \gamma$ scattering amplitudes is determined by diagrams with the smallest number of propagators. This requirement selects diagrams with the largest number of quartic $\varphi^{2} \gamma \gamma$ vertices. It is easy to understand that, for $n$-external photons, the minimal number of propagators is $n / 2-1$ if $n$ is even and $(n-1) / 2$, if $n$ is odd. Such scaling implies that

$$
\lim _{z \rightarrow \infty} \mathcal{A}_{n} \sim\left\{\begin{array}{cc}
z^{1-n / 2}, & n \text { even } \\
z^{(1-n) / 2}, & n \text { odd }
\end{array}\right.
$$

However, in reality the amplitudes decrease faster in the large $z$-limit, than indicated by Eq. (9.65) [32]. To see why this happens, consider the $n=4$

\footnotetext{
${ }^{15}$ All the momenta are considered to be outgoing.
} 
amplitude. Among six diagrams with two $\varphi^{2} \gamma \gamma$ vertices, there are three groups which correspond to definite combinations of scalar products of the polarization vectors. We write

$$
\mathcal{A}_{4}=\sum_{[i j],[l m]}\left(e_{i} \cdot e_{j}\right)\left(e_{l} \cdot e_{m}\right) A_{[i j],[l m]}+\mathcal{O}\left(z^{-2}\right),
$$

where $[i j] \neq[l m] \in\{[12],[13],[14],[23],[24],[34]\}$. Amplitude $A_{[i j],[l m]}$ is determined by the sum of two diagrams with inverse Feynman propagators that are $z q \cdot\left(k_{i}+k_{j}\right)$ and $z q \cdot\left(k_{l}+k_{m}\right)$, in the large $z$-limits. Hence, taking the sum of the two diagrams that contribute to each of these groups, we find

$$
\begin{aligned}
& \lim _{z \rightarrow \infty} A_{[i j],[l m]} \approx \frac{1}{z q \cdot\left(k_{i}+k_{j}\right)}+\frac{1}{z q \cdot\left(k_{l}+k_{m}\right)}+\mathcal{O}\left(z^{-2}\right) \\
& =\frac{q \cdot\left(k_{i}+k_{j}+k_{l}+k_{m}\right)}{z q \cdot\left(k_{i}+k_{j}\right) q \cdot\left(k_{l}+k_{m}\right)}+\mathcal{O}\left(z^{-2}\right) \\
& =-\frac{q \cdot\left(p_{a}(z)+p_{b}(z)\right)}{z q \cdot\left(k_{i}+k_{j}\right) q \cdot\left(k_{l}+k_{m}\right)}+\mathcal{O}\left(z^{-2}\right)=\mathcal{O}\left(z^{-2}\right) .
\end{aligned}
$$

The very last step follows from the fact that $q$ is transverse to the momenta of the two scalars, $q \cdot p_{a}=q \cdot p_{b}=0$. Hence, we conclude that for $n=4$, the amplitude scales as $\mathcal{A}_{4} \sim z^{-2}$ in the large- $z$ limit. It is straightforward to see how this cancellation mechanism generalizes to higher multiplicities. Consider the $n=5$ case and take all diagrams where photons $e_{1,2}$ and $e_{3,4}$ form the $\varphi^{2} \gamma \gamma$ vertices, and the photon $e_{5}$ contributes to $\varphi^{2} \gamma$ vertex. These diagrams have two propagators and, therefore, naively should scale as $z^{-2}$. However, collecting all such diagrams - that differ by the permutation of $\left(e_{1} \cdot e_{2}\right),\left(e_{3} \cdot e_{4}\right)$ and $e_{5}$, we find that they are proportional to

$$
q \cdot \sum_{i=1}^{5} k_{i}=-q \cdot\left(p_{a}+p_{b}\right)=0,
$$

which implies that $\mathcal{A}_{5} \sim z^{-3}$. The mechanism described here is generic and can be shown to be valid for arbitrary multiplicities [32]. We conclude that the tree $0 \rightarrow 2 \varphi+n \gamma$ amplitudes scale as

$$
\lim _{z \rightarrow \infty} \mathcal{A}_{n} \sim z^{-n+2} .
$$

We can use very similar arguments to understand properties of the rational part of one-loop $n$-photon scattering amplitudes, mediated by scalars. We shall compute the rational part directly following Ref. [30]. We explained this method in Section 8.3. We need to consider quadruple, triple and double cuts. The respective rational contribution due to the quadruple cut is calculated following Eq. (8.77), where the parametrization of the cut loop momentum reads $l_{q, \pm}^{\nu}=$ $V_{4}^{\nu} \pm l_{\perp} n_{4}^{\nu}+\mu n_{\epsilon}^{\nu}$. We remind the reader that $l_{q, \pm}^{2}=0$ and that $l_{\perp} \sim \mu$, in the limit of large $\mu$. To connect the calculation of the rational part with the 
large- $z$ scaling discussed previously, we note that we can take $n_{4}$ as the auxiliary quantization vector for the photons. Then, $\mu$ plays the role of $z$ and $n_{4}$ plays the role of $q$ in the preceding discussion of the tree amplitudes. Therefore, taking the large- $\mu$ limit and using Eq. (9.69), we find

$$
\lim _{\mu \rightarrow \infty}\left[A_{1} A_{2} A_{3} A_{4}\right] \sim \mu^{-n+8} .
$$

Since, as follows from Eq. (8.77), only the $\mathcal{O}\left(\mu^{4}\right)$ term in the product of four on-shell amplitudes can lead to a quadruple cut contribution to the rational part, we find that if the number of photons exceeds four, the quadruple-cut contribution to the rational part vanishes.

As the next step, we consider a triple cut. We parametrize the loop momentum as $l_{t, \pm}^{\nu}=V_{3}^{\nu}+l_{\perp}\left(t n_{\mp}^{\nu}+t^{-1} n_{ \pm}^{\nu}\right)+\mu n_{\epsilon}$. According to Eq. (8.84), we need to consider the Laurent expansion in the variable $t$ at infinity, followed by the Laurent expansion in the variable $\mu$ at infinity. Similar to the quadruple cut discussed above, $l_{\perp} \sim \mu$, in the large- $\mu$ limit. We can take $n_{ \pm}$as auxiliary quantization vectors for photons, in respective amplitudes. Using Eq. (9.69), we find that the product of three amplitudes scales as

$$
\lim _{\mu \rightarrow \infty} \lim _{t \rightarrow \infty}\left[A_{1} A_{2} A_{3}\right] \sim \mu^{6-n} t^{6-n} .
$$

According to Eq. (8.84), the rational part requires picking up the $\mathcal{O}\left(t^{0} \mu^{2}\right)$ contribution from the product of the three amplitudes and, as follows from Eq. (9.71) such terms do not exist. Finally, one can address the double-cut contribution to the rational part using similar arguments. We do not discuss this issue here. The interested reader may consult Ref. [32] where it was shown that double-cut contributions also vanish for $n \geq 6$.

\subsection{The axial anomaly}

One of the manifestations of the Adler-Bell-Jackiw axial anomaly in QED $[120,121]$ is the peculiar property of the matrix element of the divergence of the axial current $J_{\mu}^{5}=\bar{\psi} \gamma_{\mu} \gamma_{5} \psi$, where $\psi$ is the "electron" field, taken between the vacuum and the two-photon states. For massless electrons, such a matrix element reads

$$
\mathcal{M}_{A B J}=\left\langle\gamma\left(k_{1}, \lambda_{1}\right) \gamma\left(k_{2}, \lambda_{2}\right)\left|\partial^{\mu} J_{\mu}^{5}(0)\right| 0\right\rangle=\frac{e^{2}}{2 \pi^{2}} \varepsilon^{\mu \nu \lambda \rho} e_{1 \mu}^{*} k_{1 \nu} e_{2 \lambda}^{*} k_{2 \rho},
$$

where $k_{1,2}$ and $e_{1,2}$ are momenta and polarization vectors of the outgoing photons with helicities $h_{1,2}$. The matrix element $\mathcal{M}_{A B J}$ is purely rational. Below we derive $\mathcal{M}_{A B J}$ using the algorithm of $D$-dimensional unitarity.

The amplitude $M_{A B J}$ is given by the sum of two triangle Feynman diagrams with the electron loop. The matrix element is written as

$$
\begin{aligned}
\mathcal{M}_{A B J} & =\frac{i e^{2}}{(4 \pi)^{(D / 2)}} \int \frac{d^{D} l}{i \pi^{(D / 2)}} \\
& \times \operatorname{Tr}\left\{\hat{k}_{12} \Gamma_{\gamma_{5}}\left[\frac{\hat{l} \hat{e}_{1}^{*}\left(\hat{l}+\hat{k}_{1}\right) \hat{e}_{2}^{*}\left(\hat{l}+\hat{k}_{12}\right)}{l^{2}\left(l+k_{1}\right)^{2}\left(l+k_{12}\right)^{2}}\right]+(1 \leftrightarrow 2)\right\},
\end{aligned}
$$


where $k_{12}=k_{1}+k_{2}$. The external momentum and polarization vectors are fourdimensional, whereas the loop momentum and the Dirac matrices $\Gamma^{\mu}$ and the matrix $\Gamma_{\gamma_{5}}$ are continued to $D$-dimensions, following the discussion in Sect. 7 . We note that $\Gamma_{\gamma_{5}}$ in Eq. (9.73) denotes the $D$-dimensional continuation of the matrix $\gamma_{5}$. We perform such a continuation following the t'Hooft and Veltman prescription [45]. It is defined by the set of commutation relations

$$
\begin{aligned}
& \left\{\Gamma^{\mu}, \Gamma_{\gamma_{5}}\right\}=0, \text { for } \mu=0,1,2,3, \\
& {\left[\Gamma^{\mu}, \Gamma_{\gamma_{5}}\right]=0, \text { for } \mu=4, \ldots D-1 .}
\end{aligned}
$$

Eq. (9.73) defines the integrand function of the loop momentum integral but it does not define it uniquely. This is not a problem since the integral is regularized dimensionally and shifts of the loop momenta are allowed. We will exploit such shifts to simplify the computation. To this end, we split the integrand in Eq. (9.73) using the identity

$$
\hat{k}_{12} \Gamma_{\gamma_{5}}=\left(\hat{l}+\hat{k}_{12}\right) \Gamma_{\gamma_{5}}+\Gamma_{\gamma_{5}} \hat{l}-2 \Gamma_{\gamma_{5}} \hat{l}_{\epsilon}
$$

where $l_{\epsilon}^{\mu}=\left(l \cdot n_{\epsilon}\right) n_{\epsilon}^{\mu}$ is the $(D-4)$-dimensional part of the loop momentum. After the split, the trace in Eq. (9.73) gets additional terms

$$
\begin{aligned}
& \operatorname{Tr}\left\{\hat{k}_{12} \Gamma_{\gamma_{5}}\left[\frac{\hat{l} \hat{e}_{1}^{*}\left(\hat{l}+\hat{k}_{1}\right) \hat{e}_{2}^{*}\left(\hat{l}+\hat{k}_{12}\right)}{l^{2}\left(l+k_{1}\right)^{2}\left(l+k_{12}\right)^{2}}\right]+(1 \leftrightarrow 2)\right\}=\operatorname{Tr}_{1}+\operatorname{Tr}_{2}, \\
& \operatorname{Tr}_{1}=-2 \operatorname{Tr}\left\{\Gamma_{\gamma_{5}} \hat{l}_{\epsilon} \frac{\hat{l} \hat{e}_{1}^{*}\left(\hat{l}+\hat{k}_{1}\right) \hat{e}_{2}^{*}\left(\hat{l}+\hat{k}_{12}\right)}{l^{2}\left(l+k_{1}\right)^{2}\left(l+k_{12}\right)^{2}}+(1 \leftrightarrow 2)\right\}, \\
& \operatorname{Tr}_{2}=\operatorname{Tr}\left\{\Gamma_{\gamma_{5}}\left[\frac{\hat{l} \hat{e}_{1}^{*}\left(\hat{l}+\hat{k}_{1}\right) \hat{e}_{2}^{*}}{l^{2}\left(l+k_{1}\right)^{2}}+\frac{\hat{e}_{1}^{*}\left(\hat{l}+\hat{k}_{1}\right) \hat{e}_{2}^{*}\left(\hat{l}+\hat{k}_{12}\right)}{\left(l+k_{1}\right)^{2}\left(l+k_{12}\right)^{2}}\right]+(1 \leftrightarrow 2)\right\} .
\end{aligned}
$$

Fortunately, it is easy to perform shifts of the loop momenta of the type $l \rightarrow$ $l-k_{1,2}$ to show that the contribution due to $\operatorname{Tr}_{2}$ vanishes. This allows us to re-write Eq. (9.73) in a simplified form

$$
\begin{aligned}
& \mathcal{M}_{A B J}=\int \frac{d^{D} l}{i(\pi)^{(D / 2)}} \mathcal{I}_{A B J}\left(k_{1}, k_{2}, e_{1}, e_{2}, l\right), \\
& \mathcal{I}_{A B J}=\frac{-2 i e^{2}}{(4 \pi)^{(D / 2)}} \operatorname{Tr}\left\{\Gamma_{\gamma_{5}} \hat{l}_{\epsilon} \frac{\hat{l} \hat{e}_{1}^{*}\left(\hat{l}+\hat{k}_{1}\right) \hat{e}_{2}^{*}\left(\hat{l}+\hat{k}_{12}\right)}{l^{2}\left(l+k_{1}\right)^{2}\left(l+k_{12}\right)^{2}}+(1 \leftrightarrow 2)\right\} .
\end{aligned}
$$

Since the integrand $\mathcal{I}_{A B J}$ is proportional to $\hat{l}_{\epsilon}$, its cut-constructible part vanishes and its OPP parametrization becomes simple

$$
\begin{aligned}
\mathcal{I}_{A B J}(l)= & \frac{c_{1} l_{\epsilon}^{2}}{d_{0} d_{1} d_{12}}+\frac{c_{2} l_{\epsilon}^{2}}{d_{0} d_{2} d_{12}}+\frac{b_{1} l_{\epsilon}^{2}}{d_{0} d_{1}}+\frac{b_{2} l_{\epsilon}^{2}}{d_{0} d_{2}} \\
& +\frac{b_{3} l_{\epsilon}^{2}}{d_{1} d_{12}}+\frac{b_{4} l_{\epsilon}^{2}}{d_{2} d_{12}}+\frac{b_{5} l_{\epsilon}^{2}}{d_{0} d_{12}} .
\end{aligned}
$$


We use $d_{0}=l^{2}, d_{1}=\left(l+k_{1}\right)^{2}, d_{2}=\left(l+k_{2}\right)^{2}$ and $d_{12}=\left(l+k_{12}\right)^{2}$ in Eq. (9.78). The general OPP parameterization Eq. (5.16) for non-cut constructible terms would contain extra terms trilinear in the loop momentum that are not present in Eq. (9.78). The fact that these terms are absent will be justified a posteriori. Although we work under the assumption that electrons are massless, we note that all the manipulations we did up to now remain valid also for massive electrons. ${ }^{16}$ In the massless electron case some of the bubble integrals are scaleless and therefore vanish, but the residues of the corresponding integrands do not vanish and are, in fact, mass-independent. We will show below that $c_{1}=c_{2}$ and $b_{i=1, . .5}=0$.

In the case of closed fermion loops the Dirac algebra has to be performed in six dimensions with five-dimensional loop momentum $l=l_{(4)}+l_{\epsilon}$, where using the notation $\left(l_{0}, l_{1}, l_{2}, l_{3}, l_{4}, l_{5}\right)$ we have that $l_{\epsilon}=(0,0,0,0, \mu, 0)$ and $l_{\epsilon}^{2}=-\mu^{2}$. As explained in Sect. 7.2, for six-dimensional Dirac matrices we use the simple representation

$$
\begin{aligned}
& \Gamma^{0}=\left(\begin{array}{cc}
\gamma^{0} & \mathbf{0} \\
\mathbf{0} & \gamma^{0}
\end{array}\right), \quad \Gamma^{i=1,2,3}=\left(\begin{array}{cc}
\gamma^{i} & \mathbf{0} \\
\mathbf{0} & \gamma^{i}
\end{array}\right), \\
& \Gamma^{4}=\left(\begin{array}{cc}
\mathbf{0} & \gamma_{5} \\
-\gamma_{5} & \mathbf{0}
\end{array}\right), \quad \Gamma^{5}=\left(\begin{array}{cc}
\mathbf{0} & i \gamma_{5} \\
i \gamma_{5} & \mathbf{0}
\end{array}\right), \quad \Gamma_{\gamma_{5}}=\left(\begin{array}{cc}
\gamma_{5} & \mathbf{0} \\
\mathbf{0} & \gamma_{5}
\end{array}\right) .
\end{aligned}
$$

Note that for our choice of $l, \Gamma^{5}$ never appears in Eq. (9.77). Finally, we choose a special reference frame where

$$
\begin{gathered}
k_{12}=(m, 0,0,0,0,0), \quad k_{1,2}=\left(\frac{m}{2}, \pm \frac{m}{2}, 0,0,0,0\right), \\
e_{1,2}^{*}=\frac{1}{\sqrt{2}}(0,0,1, \pm i, 0,0), \quad l_{\perp}=\alpha e_{1}^{*}+\beta e_{2}^{*} .
\end{gathered}
$$

With this choice of the polarization vectors, it is clear that $e_{i}^{*} k_{j}=0,(i=1,2)$. The coefficients $c_{1}, c_{2}, b_{1}, \ldots b_{5}$ can be obtained by evaluating triple cuts and double cuts on both sides of Eq. (9.78).

We begin by considering the triple cut specified by the condition $d_{0}=d_{1}=$ $d_{12}=0$. Decomposing the loop momentum on the cut as

$$
l_{c_{1}}^{\mu}=x_{1} k_{1}^{\mu}+x_{2} k_{2}^{\mu}+\tilde{l}^{\mu}, \quad \tilde{l}^{\mu}=l_{\perp}^{\mu}+l_{\epsilon}^{\mu},
$$

we find that $x_{1}=-1, x_{2}=0$ and $\tilde{l}^{2}=l_{\perp}^{2}+l_{\epsilon}^{2}=0$. Taking the $d_{0}, d_{1}, d_{12}$ residue of the left hand side of Eq. (9.78) we obtain

$$
\begin{aligned}
& \left.\operatorname{Res}\left(\mathcal{I}_{A B J}\right)\right|_{d_{0}=d_{1}=d_{12}=0}= \\
& \frac{-2 i e^{2}}{(4 \pi)^{(D / 2)}} \operatorname{Tr}\left\{\Gamma_{\gamma_{5}} \hat{l}_{\epsilon}\left(\hat{l}_{\perp}+\hat{l}_{\epsilon}-\hat{k}_{1}\right) \hat{e}_{1}^{*}\left(\hat{l}_{\perp}+\hat{l}_{\epsilon}\right) \hat{e}_{2}^{*}\left(\hat{l}_{\perp}+\hat{l}_{\epsilon}+\hat{k}_{2}\right)\right\} .
\end{aligned}
$$

\footnotetext{
${ }^{16}$ In the massive electron case, the divergence of the axial current involves a canonical term $\partial_{\mu} J_{5}^{\mu}=2 m \bar{\psi} \gamma_{5} \psi$, which should be treated separately.
} 
It follows from Eq. (9.82) that the triple cut residue is the fourth-order polynomial in $l_{\epsilon}$. However, it is easy to argue that only limited number of terms can contribute to the trace. Indeed, for our choice of the loop momentum, $\hat{l}_{\epsilon}$ is proportional to $\Gamma^{4}$ in Eq. (9.79) while all other terms in Eq. (9.82) are linear combinations of $\Gamma^{0,1,2,3}$. Since the former is block off-diagonal while the latter are block-diagonal, terms with odd number of $l_{\epsilon}$ 's do not contribute to the trace. Moreover, for the trace in Eq. (9.82) to be non-zero, at least four $\Gamma$ matrices are needed in addition to $\Gamma_{\gamma_{5}}$. Since $\hat{l}_{\epsilon}$ anticommutes with all other matrices of the trace, the term with four $\hat{l}_{\epsilon}$ vanishes. We conclude that the only term that, perhaps, contributes to the trace is quadratic in $\hat{l}_{\epsilon}$. This justifies the form of the OPP parameterization in Eq. (9.78). Finally, because $l_{\perp}$ can be written as a linear combination of $e_{1}^{*}$ and $e_{2}^{*}$, only terms that contain two $\hat{l}_{\epsilon}$ and no $l_{\perp}$ terms give non-vanishing contributions. Taking all this into account, we arrive at a simple expression for the trace and the residue

$$
\left.\operatorname{Res}\left(\mathcal{I}_{A B J}\right)\right|_{d_{0}=d_{1}=d_{12}=0}=\frac{2 i e^{2}}{(4 \pi)^{(D / 2)}} \operatorname{Tr}\left\{\Gamma_{\gamma_{5}} \hat{l}_{\epsilon} \hat{k}_{1} \hat{e}_{1}^{*} \hat{l}_{\epsilon} \hat{e}_{2}^{*} \hat{k}_{2}\right\}
$$

Since the residue of the right hand side in Eq. (9.78) is $c_{1} l_{\epsilon}^{2}$ we derive the value of the $c_{1}$ coefficient

$$
c_{1}=-\frac{2^{D / 2+1} e^{2}}{(4 \pi)^{\frac{D}{2}}} \varepsilon^{\mu \nu \lambda \rho} e_{1 \mu}^{*} k_{1 \nu} e_{2 \lambda}^{*} k_{2 \rho} .
$$

Finally, because of the $1 \leftrightarrow 2$ symmetry, we find $c_{2}=c_{1}$.

We next proceed to the double cuts. Apart from obvious changes in the physical and transverse spaces, the only new feature is that on the right hand side of Eq. (9.78) we get the double cut contribution also from the triple pole terms. We illustrate the calculation of the double-pole terms taking $d_{0}=d_{1}=0$, as an example. Although this is a double cut, the reference momentum is light-like $k_{1}^{2}=0$, so the parametrization is subtle. We parametrize the loop momentum on the double-cut as

$$
l_{b_{1}}^{\mu}=x_{1} k_{1}^{\mu}+x_{2} k_{2}^{\mu}+\tilde{l}^{\mu},
$$

and use $l^{2}=0, l \cdot k_{1}=0$ to find $x_{2}=0$ and $\tilde{l}^{2}=0$, while $x_{1}$ is unconstrained. We compute the $d_{0}, d_{1}$ residue of the left-hand side of Eq. (9.78) using the expression in Eq. (9.77). We obtain

$$
\begin{aligned}
& \left.\operatorname{Res}\left(\mathcal{I}_{A B J}\right)\right|_{d_{0}=d_{1}=0}=\frac{-2 i e^{2}}{(4 \pi)^{\frac{D}{2}}} \frac{1}{\left(l+k_{12}\right)^{2}} \operatorname{Tr}\left\{\Gamma_{\gamma_{5}} \hat{l}_{\epsilon}\left(\hat{l}_{\perp}+\hat{l}_{\epsilon}+x_{1} \hat{k}_{1}\right)\right. \\
& \left.\times \hat{e}_{1}^{*}\left(\hat{l}_{\perp}+\left(1+x_{1}\right) \hat{k}_{1}+\hat{l}_{\epsilon}\right) \hat{e}_{2}^{*}\left(\hat{l}_{\perp}+\hat{l}_{\epsilon}+x_{1} \hat{k}_{1}+\hat{k}_{12}\right)\right\} .
\end{aligned}
$$

Similar to the triple-cut case considered earlier, only terms quadratic in $l_{\epsilon}$ contribute. The non-vanishing terms are proportional to $k_{2}$ and, after some algebra, 
we find that all terms proportional to $x_{1}$ cancel and the result is simply expressed through the $c_{1}$ coefficient in Eq. (9.84)

$$
\left.\operatorname{Res}\left(\mathcal{I}_{A B J}\right)\right|_{\left(d_{0}=d_{1}=0\right)}=c_{1} \frac{l_{\epsilon}^{2}}{\left(l+k_{12}\right)^{2}} .
$$

Since the $\left(d_{0}, d_{1}\right)$-residue in Eq. $(9.78)$ is $c_{1} l_{\epsilon}^{2} / d_{12}+b_{1}$, we find $b_{1}=0$. A similar calculation proves that other bubble coefficients also vanish. To obtain the final result, we need the value of the triangle integral, (see Eq. (B.6)),

$$
\int \frac{\mathrm{d}^{D} l}{\pi^{D / 2}} \frac{l_{\epsilon}^{2}}{d_{0} d_{1} d_{2}}=-\frac{i}{2}+\mathcal{O}(D-4),
$$

and the value of the coefficient $c_{1}$ from Eq. (9.84). By adding the two triangle contributions and setting $D=4$, we obtain the anomalous amplitude $\mathcal{M}_{A B J}$ shown in Eq. (9.72).

\subsection{Calculation of one-loop corrections to $\bar{q} q g g$}

We will illustrate the analytic methods of Section 8 by considering a specific case for a simple $2 \rightarrow 2$ process. As our example we will describe the calculation of a particular one-loop primitive amplitude for $\bar{q} q g g$ scattering.

From Eq. (6.37) we have the color decomposition for the tree graph amplitude for this process,

$$
\begin{aligned}
\mathcal{A}_{4}^{\text {tree }}\left(\bar{q}_{1}, q_{2}, g_{3}, g_{4}\right)=g_{s}^{2} & {\left[\left(T^{a_{3}} T^{a_{4}}\right)_{i_{2} \bar{\imath}_{1}} m_{4}\left(\bar{q}_{1}, q_{2}, g_{3}, g_{4}\right)\right.} \\
& \left.+\left(T^{a_{4}} T^{a_{3}}\right)_{i_{2} \bar{\imath}_{1}} m_{4}\left(\bar{q}_{1}, q_{2}, g_{4}, g_{3}\right)\right] .
\end{aligned}
$$

Further, Eq. (6.50) gives the decomposition of the full one-loop amplitude in terms of primitive amplitudes

$$
\begin{aligned}
& \mathcal{A}_{4}^{1-\text { loop }}\left(\bar{q}_{1}, q_{2}, g_{3}, g_{4}\right)=g_{s}^{4} c_{\Gamma} \\
& \times \sum_{P(3,4)}\left[\left(T^{x_{2}} T^{x_{1}}\right)_{i_{2} \bar{\imath}_{1}}\left(F^{a_{3}} F^{a_{4}}\right)_{x_{1} x_{2}} \tilde{m}_{4}^{(1)}\left(\bar{q}_{1}, q_{2}, g_{4}, g_{3}\right)\right. \\
& \quad+\left(T^{x_{2}} T^{a_{3}} T^{x_{1}}\right)_{i_{2} \overline{1}_{1}}\left(F^{a_{4}}\right)_{x_{1} x_{2}} \tilde{m}_{4}^{(1)}\left(\bar{q}_{1}, g_{3}, q_{2}, g_{4}\right) \\
& \left.\quad+\left(T^{x_{1}} T^{a_{3}} T^{a_{4}} T^{x_{1}}\right)_{i_{2} \bar{\tau}_{1}} \tilde{m}_{4}^{(1)}\left(\bar{q}_{1}, g_{4}, g_{3}, q_{2}\right)\right] .
\end{aligned}
$$

For simplicity, we neglect diagrams containing fermion loops in this discussion. By using the identities Eq. (6.1) and the crossing relation, Eq. (6.51), we can write the one-loop amplitude, Eq. (9.90), in the form in which it would appear 
after full reduction of the color matrices,

$$
\begin{aligned}
\mathcal{A}_{4}\left(\bar{q}_{1}, q_{2}, g_{3}, g_{4}\right)=g_{s}^{4} c_{\Gamma} & {\left[N_{c}\left(T^{a_{3}} T^{a_{4}}\right)_{i_{2} \bar{\imath}_{1}} A_{4 ; 1}\left(\bar{q}_{1}, q_{2}, g_{3}, g_{4}\right)\right.} \\
+ & N_{c}\left(T^{a_{4}} T^{a_{3}}\right)_{i_{2} \bar{\nu}_{1}} A_{4 ; 1}\left(\bar{q}_{1}, q_{2}, g_{4}, g_{3}\right) \\
+ & \left.\operatorname{Tr}\left(T^{a_{2}} T^{a_{3}}\right) \delta_{i_{2} \bar{\imath}_{1}} A_{4 ; 3}\left(\bar{q}_{1}, q_{2}, g_{3}, g_{4}\right)\right] .
\end{aligned}
$$

In terms of the primitive amplitudes, the amplitudes $A_{4 ; j}$ are given as,

$$
\begin{aligned}
A_{4 ; 1}\left(\bar{q}_{1}, q_{2}, g_{3}, g_{4}\right) & =\tilde{m}_{4}^{(1)}\left(\bar{q}_{1}, q_{2}, g_{3}, g_{4}\right)-\frac{1}{N_{c}^{2}} \tilde{m}_{4}^{(1)}\left(\bar{q}_{1}, g_{4}, g_{3}, q_{2}\right) \\
A_{4 ; 3}\left(\bar{q}_{1}, q_{2}, g_{3}, g_{4}\right) & =\tilde{m}_{4}^{(1)}\left(\bar{q}_{1}, q_{2}, g_{4}, g_{3}\right)+\tilde{m}_{4}^{(1)}\left(\bar{q}_{1}, g_{4}, g_{3}, q_{2}\right) \\
& +\tilde{m}_{4}^{(1)}\left(\bar{q}_{1}, g_{4}, q_{2}, g_{3}\right)+\tilde{m}_{4}^{(1)}\left(\bar{q}_{1}, q_{2}, g_{3}, g_{4}\right) \\
& +\tilde{m}_{4}^{(1)}\left(\bar{q}_{1}, g_{3}, g_{4}, q_{2}\right)+\tilde{m}_{4}^{(1)}\left(\bar{q}_{1}, g_{3}, q_{2}, g_{4}\right)
\end{aligned}
$$

In this Section we shall perform the calculation of $\tilde{m}_{4}^{(1)}\left(\bar{q}_{1}, g_{4}, g_{3}, q_{2}\right)$, the color-suppressed primitive amplitude in Eq. (9.92). As a first step we note that the singular behavior of this primitive amplitude can be read off from Eq. (6.85),

$$
\tilde{m}_{4}^{(1)}\left(\bar{q}_{1}, g_{4}, g_{3}, q_{2}\right)=-m_{4}\left(\bar{q}_{1}, q_{2}, g_{3}, g_{4}\right)\left[\left(\frac{1}{\epsilon^{2}}+\frac{1}{\epsilon}\left(\frac{3}{2}+L_{12}\right)\right)\right]+\mathcal{O}\left(\epsilon^{0}\right),
$$

where $L_{12}=\ln \left(\mu^{2} /\left(-s_{12}-i 0\right)\right), s_{12}=2 p_{1} \cdot p_{2}$.

The amplitude $\tilde{m}_{4}^{(1)}$ can be expanded in terms of scalar integrals defined in Eq. (2.6). In the notation of Passarino and Veltman, the scalar integrals that can contribute are the box integral, $D_{0}\left(p_{1}, p_{2}, p_{3}, 0,0,0,0\right)$, and any scalar integral that can be obtained from it by removing propagators. The relevant integrals are

$$
\begin{aligned}
I_{4}\left(0,0,0,0 ; s_{12}, s_{23} ; 0,0,0,0\right) & \sim D_{0}\left(p_{1}, p_{2}, p_{3}, 0,0,0,0\right), \\
I_{3}\left(0,0, s_{12} ; 0,0,0\right) & \sim C_{0}\left(p_{1}, p_{2}, 0,0,0\right)=C_{0}\left(p_{12}, p_{3}, 0,0,0\right), \\
I_{3}\left(0,0, s_{23} ; 0,0,0\right) & \sim C_{0}\left(p_{2}, p_{3}, 0,0,0\right)=C_{0}\left(p_{1}, p_{23}, 0,0,0\right), \\
I_{2}\left(s_{12}, 0,0\right) & \sim B_{0}\left(p_{12}, 0,0\right), \\
I_{2}\left(s_{23}, 0,0\right) & \sim B_{0}\left(p_{23}, 0,0\right) .
\end{aligned}
$$

The scaleless integrals $B_{0}\left(p_{i}, 0,0\right), A_{0}(0)$ are equal to zero in dimensional regularization and we do not consider them. The general expansion of the amplitude in terms of master integrals can thus be written as,

$$
\begin{aligned}
& -i \tilde{m}_{4}^{(1)}\left(\bar{q}_{1}, g_{4}, g_{3}, q_{2}\right)=\tilde{d}_{0} I_{4}\left(0,0,0,0 ; s_{12}, s_{23} ; 0,0,0,0\right) \\
& \quad+\tilde{c}_{0}^{(12)} I_{3}\left(0,0, s_{12} ; 0,0,0\right)+\tilde{c}_{0}^{(23)} I_{3}\left(0,0, s_{23} ; 0,0,0\right) \\
& \quad+\tilde{b}_{0}^{(12)} I_{2}\left(s_{12} ; 0,0\right)+\tilde{b}_{0}^{(23)} I_{2}\left(s_{23} ; 0,0\right)+\mathcal{R}
\end{aligned}
$$


where $\mathcal{R}$ stands for the rational term. In the following subsections we shall illustrate the calculation of the coefficients of the master integrals. Scalar integrals that appear in Eq. (9.96) are tabulated in Ref. [54] and are given in Appendix E for completeness.

Since the divergences of the amplitude $\tilde{m}_{4}^{(1)}\left(\bar{q}_{1}, g_{4}, g_{3}, q_{2}\right)$ and of all the scalar integrals in Eq. (9.96) are known and since all reduction coefficients are $\epsilon$-independent, we can find relations between various reduction coefficients by requiring that coefficients of $1 / \epsilon^{2}, 1 / \epsilon, L_{12} / \epsilon$ and $L_{23} / \epsilon$ on both sides in Eq. (9.96) coincide. These relations are

$$
\begin{aligned}
& \tilde{b}_{0}^{(12)}+\tilde{b}_{0}^{(23)}-\frac{3}{2} i m_{4}\left(\bar{q}_{1}, q_{2}, g_{3}, g_{4}\right)=0 \\
& 2 \frac{\tilde{d}_{0}}{s_{12} s_{23}}+\frac{\tilde{c}_{0}^{(12)}}{s_{12}}-i m_{4}\left(\bar{q}_{1}, q_{2}, g_{3}, g_{4}\right)=0 \\
& 2 \frac{\tilde{d}_{0}}{s_{12} s_{23}}+\frac{\tilde{c}_{0}^{(23)}}{s_{23}}=0 .
\end{aligned}
$$

As follows from Eq. (9.96), there are six unknowns and three equations, cf. Eq. (9.97), that constrain them. It is possible to use these relations to express the two triangle coefficients in terms of box and bubble coefficients. Therefore the full calculation reduces to the calculation of the box reduction coefficient $\tilde{d}_{0}$, only one of the two bubble coefficients, $\tilde{b}_{0}^{(12)}$ and the rational part $\mathcal{R}$. Substituting the relations Eq. (9.97) into Eq. (9.96) we obtain,

$$
\begin{aligned}
& -i \tilde{m}_{4}^{(1)}\left(\bar{q}_{1}, g_{4}, g_{3}, q_{2}\right)=\tilde{d}_{0}\left[I_{4}\left(0,0,0,0 ; s_{12}, s_{23} ; 0,0,0,0\right)\right. \\
- & \left.\frac{2}{s_{23}} I_{3}\left(0,0, s_{12} ; 0,0,0\right)-\frac{2}{s_{12}} I_{3}\left(0,0, s_{23} ; 0,0,0\right)\right] \\
+ & i m_{4}\left(\bar{q}_{1}, q_{2}, g_{3}, g_{4}\right)\left[s_{12} I_{3}\left(0,0, s_{12} ; 0,0,0\right)+\frac{3}{2} I_{2}\left(s_{12} ; 0,0\right)\right] \\
+ & \tilde{b}_{0}^{(23)}\left[I_{2}\left(s_{23} ; 0,0\right)-I_{2}\left(s_{12} ; 0,0\right)\right]+\mathcal{R} .
\end{aligned}
$$

The calculation of box coefficients

The box coefficient can be calculated by applying Eq. (8.1) and using analytic results for the amplitudes at the corners of the boxes to compute $\tilde{d}_{0}$. A simple example will illustrate the power of the method. Consider the diagram shown in Fig. 9.4. Momentum assignments for the internal lines are $l_{3}=l_{0}-p_{3}, l_{23}=$ $l_{0}-p_{2}-p_{3}, l_{4}=l_{0}+p_{4}$ and all external momenta are taken to be outgoing.

To parametrize the loop momentum in such a way that four on-shell conditions are easily solved, we choose to expand the vector $l_{0}$, (which is flanked on either side by two massless momenta $p_{3}$ and $p_{4}$ ), in terms of $p_{3}$ and $p_{4}$ as well as two complex momenta formed from spinors of $p_{3}$ and $p_{4}$. We write

$$
l_{0}^{\mu}=\alpha p_{3}^{\mu}+\beta p_{4}^{\mu}+\gamma \epsilon_{34}^{\mu}+\delta \epsilon_{43}^{\mu},
$$




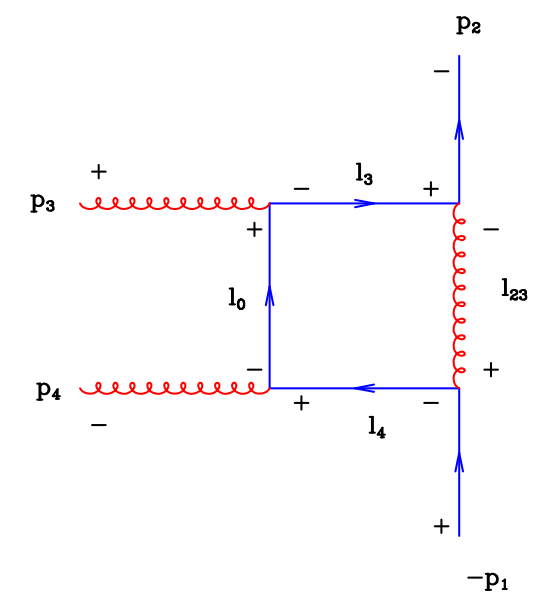

Figure 9.4: A box contribution to the amplitude $\tilde{m}^{(1)}\left(\bar{q}_{1}^{+}, g_{4}^{-}, g_{3}^{+}, q_{2}^{-}\right)$

where $^{17}$

$$
\epsilon_{i j}=\frac{1}{2}\left\langle i-\left|\gamma^{\mu}\right| j-\right\rangle=\frac{1}{2}\left\langle i\left|\gamma^{\mu}\right| j\right] .
$$

These vectors are light-like and transverse to the momenta $p_{i, j}$. In particular,

$$
\epsilon_{i j} \cdot \epsilon_{i j}=0, \quad \epsilon_{i j} \cdot \epsilon_{j i}=-\frac{s_{i j}}{2},
$$

where $s_{i j}=2 p_{i} \cdot p_{j}$. Using the mass shell conditions for three internal lines $l_{3}^{2}=l_{4}^{2}=l_{0}^{2}$, we find $\alpha=0, \beta=0$ and $\gamma \delta=0$. We take $\delta=0$ and justify this choice in the next Section. The last on-shell condition $l_{23}^{2}=0$ fixes the value of $\gamma$,

$$
-\gamma\left\langle 3\left|\not p_{2}+\not p_{3}\right| 4\right]+2 p_{2} \cdot p_{3}=-\gamma\langle 32\rangle[24]+\langle 32\rangle[23]=0, \Rightarrow \gamma=\frac{[23]}{[24]} .
$$

\section{Box coefficients for $q g g \bar{q}$}

The box coefficients are determined by the quadruple cuts according to Eq. (8.1). For the case $q g g \bar{q}$ the on-shell tree amplitudes at the four corners of the diagram are three-parton vertices. The analytic expressions for the relevant three parton vertices are given in spinor notation in Appendix D.2. These three-point vertices, calculated for three complex momenta, $p_{i}, p_{j}, p_{k}$, are subject to the mass shell constraint that either $\langle i j\rangle=\langle i k\rangle=\langle j k\rangle=0$ or $[i j]=[i k]=[j k]=0$.

We consider first the case where the external gluons have the same helicity. This helicity amplitude vanishes because we would have three-point vertices

\footnotetext{
${ }^{17}$ Our notations for the spinor-helicity variables are summarized in Appendix D.
} 
with helicity assignments (++-) both at the vertex where $p_{3}$ flows out and at the vertex where $p_{4}$ flows out. This implies that the two vertices are

$$
\frac{\left[3 l_{0}\right]^{2}}{\left[l_{3} l_{0}\right]} \times \frac{\left[4 l_{4}\right]^{2}}{\left[l_{0} l_{4}\right]}
$$

or consequently that both $\left\langle l_{0} 3\right\rangle=0$ and $\left\langle l_{0} 4\right\rangle=0$ which cannot simultaneously be satisfied for generic external momenta $p_{3}$ and $p_{4}$. This helicity amplitude thus has a vanishing contribution to the coefficient of this box integral.

Next consider the case where the gluons have opposite helicities as shown in Fig. 9.4. The box coefficient is constructed from the product of four three-point amplitudes. We find

$$
\tilde{d}_{0}\left(\bar{q}_{1}^{+}, g_{4}^{-}, g_{3}^{+}, q_{2}^{-}\right)=\frac{1}{2} \frac{\left\langle l_{23} 2\right\rangle^{2}}{\left\langle 2 l_{3}\right\rangle} \times \frac{\left[3 l_{0}\right]^{2}}{\left[l_{3} l_{0}\right]} \times \frac{\left\langle l_{0} 4\right\rangle^{2}}{\left\langle l_{0} l_{4}\right\rangle} \times \frac{\left[1 l_{23}\right]^{2}}{\left[1 l_{4}\right]} .
$$

To simplify this expression, we use e.g.

$$
\left[3 l_{0}\right]\left\langle l_{0} 4\right\rangle=\left[3\left|\hat{l}_{0}\right| 4\right\rangle .
$$

In order for this quantity to be non-vanishing, we must have $\gamma \neq 0$ and hence $\delta=0$. This justifies setting $\delta=0$ in our parametrization of the momentum $l_{0}$. Collecting terms we may write Eq. (9.104) as

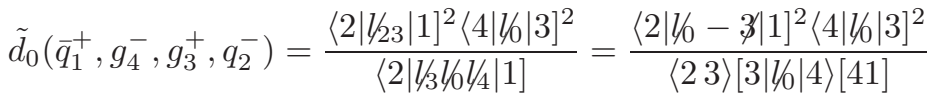

$$
\begin{aligned}
& =\frac{(\gamma\langle 23\rangle[41]-\langle 23\rangle[31])^{2} \gamma\langle 43\rangle[43]}{\langle 23\rangle[41]},
\end{aligned}
$$

where we have used mass shell constraints and made the substitution $\ell_{0}=$ $\gamma|3\rangle[4 \mid$. Finally, using $\gamma=[23] /[24]$ from Eq. (9.102) we obtain

$$
\begin{aligned}
\tilde{d}_{0}\left(\bar{q}_{1}^{+}, g_{4}^{-}, g_{3}^{+}, q_{2}^{-}\right) & =\frac{1}{2} \frac{\langle 23\rangle^{2}([23][41]-[24][31])^{2}[23]\langle 43\rangle[43]}{\langle 23\rangle[24]^{3}[41]} \\
& =\frac{1}{2} \frac{\langle 23\rangle[21]^{2}[23]\langle 43\rangle[43]^{3}}{[24]^{3}[41]} \\
& =\frac{1}{2} \frac{\langle 23\rangle\langle 21\rangle[21]^{2}[23]\langle 43\rangle[43]^{3}}{[24]^{3}[41]\langle 21\rangle} \\
& =\frac{1}{2} \frac{\langle 21\rangle[21]^{2}[23]\langle 43\rangle[43]^{2}}{[24]^{3}} \\
& =\frac{1}{2} \frac{s_{12}^{2} s_{23}[21][43]}{\langle 23\rangle[24]^{3}}=\frac{1}{2} \frac{s_{12} s_{23}[21]^{2}[34]^{2}}{[24]^{3}[41]} .
\end{aligned}
$$

\section{General methods for bubble coefficients}

In Section 8.2 we discussed the technique to obtain analytic results for bubble reduction coefficients. Here we will apply this technique to compute the 
reduction coefficient $\tilde{b}_{0}^{12}$ in Eq. (9.96). It turns out that for computations that employ spinor-helicity variables, it is convenient to write the momentum flowing into the bubble as a sum of two light-like vectors. Therefore, we will use the momentum parametrization shown in Eq. (8.53), but we identify

$$
q_{1}=-p_{3}-p_{4}, \quad n_{2}=\frac{\left(p_{3}-p_{4}\right)}{\sqrt{-q_{1}^{2}}}, \quad n_{+}=\frac{\varepsilon_{34}}{\sqrt{-q_{1}^{2}}}, \quad n_{-}=\frac{\varepsilon_{43}}{\sqrt{-q_{1}^{2}}} .
$$

With this parametrization, the momentum on the double cut reads

$$
l^{\mu}=\frac{1}{1+z \bar{z}}\left(p_{3}^{\mu}+z \bar{z} p_{4}^{\mu}+\varepsilon_{43}^{\mu} z+\varepsilon_{34}^{\mu} \bar{z}\right) .
$$

According to Mastrolia's method, explained in Section 8.2, we need to compute the double cut residue $f(z, \bar{z})$, see Eq. (8.56), find the anti-derivative of the function $f(z, \bar{z}) /(1+z \bar{z})^{2}$ with respect to $\bar{z}$ and compute the $z$-residues of this anti-derivative in the entire complex $z$-plane.

To get used to this parametrization, we consider how various integrands $f(z, \bar{z}) /(1+z \bar{z})^{2}$ appear when expressed in terms of $z$ and $\bar{z}$. We write schematically

$$
\begin{aligned}
\frac{1}{l^{2}\left(l-p_{3}-p_{4}\right)^{2}} & \rightarrow \frac{1}{(1+z \bar{z})^{2}} \\
\frac{1}{l^{2}\left(l-p_{3}\right)^{2}\left(l-p_{3}-p_{4}\right)^{2}} & \rightarrow \frac{-1}{2 p_{3} \cdot p_{4}} \frac{1}{(1+z \bar{z}) z \bar{z}} \\
\frac{l^{\mu}}{l^{2}\left(l-p_{3}\right)^{2}\left(l-p_{3}-p_{4}\right)^{2}} & \rightarrow \frac{-1}{2 p_{3} \cdot p_{4}} \frac{p_{3}^{\mu}+z \bar{z} p_{4}^{\mu}+z \varepsilon_{43}+\bar{z} \varepsilon_{34}}{(1+z \bar{z})^{2} z \bar{z}}
\end{aligned}
$$

Obtaining the primitives with respect to $\bar{z}$ we get

$$
\begin{aligned}
\frac{1}{l^{2}\left(l-p_{3}-p_{4}\right)^{2}} & \rightarrow-\frac{1}{z(1+z \bar{z})} \\
\frac{1}{l^{2}\left(l-p_{3}\right)^{2}\left(l-p_{3}-p_{4}\right)^{2}} & \rightarrow-\frac{1}{2 p_{3} \cdot p_{4}} \frac{\ln (\bar{z})-\ln (z \bar{z}+1)}{z} \\
\frac{l^{\mu}}{l^{2}\left(l-p_{3}\right)^{2}\left(l-p_{3}-p_{4}\right)^{2}} & \rightarrow \frac{1}{2 p_{3} \cdot p_{4}} \frac{\left(p_{4}^{\mu}-p_{3}^{\mu}\right)-z \varepsilon_{43}^{\mu}+\frac{1}{z} \varepsilon_{34}^{\mu}}{(1+z \bar{z}) z} . \\
& + \text { logarithmic terms }
\end{aligned}
$$

Discarding the logarithmic terms and calculating residues with respect to $z$, we find the expected contributions to the bubble coefficients coming from the scalar bubble and the rank-one triangle.

Application to $\tilde{b}_{0}^{(12)}\left(\bar{q}_{1}^{+}, g_{4}^{-}, g_{3}^{+}, q_{2}^{-}\right)$.

Now we turn to the concrete physical example, shown in Fig. 9.5. First we should write down amplitudes for the tree level processes on either side of the two particle cut. The computation of these amplitudes is discussed in Appendix D. 


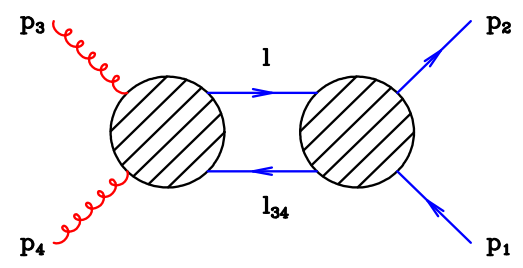

Figure 9.5: The double cut of the amplitude $M^{(1)}\left(1_{\bar{q}}^{+}, 2_{q}^{-}, 3_{g}^{+}, 4_{g}^{-}\right)$

Specifically, the amplitude on the left hand side of the cut, $M_{L}$, is the amplitude for opposite helicity gluon quark scattering, Eq. (D.35), which can also be written as

$$
i M_{L}=m_{4}\left(\bar{q}_{1}^{-}, q_{2}^{+}, g_{3}^{+}, g_{4}^{-}\right)=i \frac{\langle 42\rangle^{3}}{\langle 23\rangle\langle 34\rangle\langle 12\rangle} .
$$

In addition, we shall need four-quark scattering, derived in Eq. (D.40),

$$
i M_{R}=m_{4}\left(\bar{q}_{1}^{+}, q_{3}^{-}, \bar{Q}_{4}^{+}, Q_{2}^{-}\right)=i \frac{[14]^{2}}{[24][31]} .
$$

Now we form the combination as indicated in Fig. 9.5. This corresponds to inserting Eq. (9.112) with $1 \rightarrow-l_{34}, 2 \rightarrow l$, and inserting Eq. (9.113) with $3 \rightarrow l_{34}, 4 \rightarrow-l$ where $l_{34}=l+p_{3}+p_{4}$. We find

$$
i M_{L}\left(-l_{34}, l, p_{3}, p_{4}\right) \times i M_{R}\left(p_{1}, p_{2}, l_{34},-l\right)=-\frac{\langle 4 l\rangle^{3}}{\langle l 3\rangle\langle 34\rangle\left\langle l_{34} l\right\rangle} \times \frac{[1 l]^{2}}{[2 l]\left[1 l_{34}\right]} .
$$

We eliminate $l_{34}$ using the momentum conservation relation

$$
\left.\left\langle l l_{34}\right\rangle\left[l_{34} 1\right]=\langle l|\not 3+\not|| 1\right]=-\langle l 2\rangle[21],
$$

and obtain

$$
i M_{L} \times i M_{R}=\frac{1}{\langle 34\rangle[21]} \times \frac{\langle 4 l\rangle^{3}[1 l]^{2}}{\langle 2 l\rangle[2 l]\langle 3 l\rangle} .
$$

Putting in the integration measure and rescaling the loop momentum as

$$
l^{\mu}=\frac{1}{1+z \bar{z}} \lambda^{\mu}, \Rightarrow \lambda^{\mu}=p_{3}^{\mu}+z \bar{z} p_{4}^{\mu}+\frac{z}{2}\left\langle 4\left|\gamma^{\mu}\right| 3\right]+\frac{\bar{z}}{2}\left\langle 3\left|\gamma^{\mu}\right| 4\right],
$$

we derive an integral representation for the $\tilde{b}_{0}^{(12)}$ coefficient

$$
\begin{gathered}
\tilde{b}_{0}^{(12)}=\int d^{4} l \delta^{+}\left(l^{2}\right) \delta^{+}\left((l-P)^{2}\right) i M_{L} \times i M_{R} \\
=-\int \frac{d z d \bar{z}}{(1+z \bar{z})^{2}} \frac{1}{\langle 34\rangle[21]} \times \frac{\langle 4 \lambda\rangle^{3}[1 \lambda]^{2}}{(1+z \bar{z})[2 \lambda]\langle 2 \lambda\rangle\langle 3 \lambda\rangle}
\end{gathered}
$$


To proceed further, we shall first find the primitive with respect to $z$. To accomplish this, we rewrite Eq. (9.118) as

$$
\tilde{b}_{0}^{(12)}=-\frac{s_{12}^{3}}{\langle 34\rangle[21]} \times \oint_{C} d \bar{z} \int d z \frac{\langle 4 \lambda\rangle^{3}[1 \lambda]^{2}}{\langle\lambda|P| \lambda]^{3}[2 \lambda]\langle 2 \lambda\rangle\langle 3 \lambda\rangle},
$$

where momentum $P=p_{3}+p_{4}$ and

$$
\langle\lambda|P| \lambda]=P^{2}(1+z \bar{z}) .
$$

The $z$-dependence in Eq. (9.119) is now hidden in the $\lambda$-spinors, so we examine all $\lambda$-dependent terms in Eq. (9.119). We write them as

$$
A=\frac{\langle 4 \lambda\rangle^{3}}{\langle\lambda|P| \lambda]^{3}\langle 2 \lambda\rangle\langle 3 \lambda\rangle} .
$$

To simplify Eq. (9.121), we note that

$$
\mid \lambda]=\mid 3]+\bar{z} \mid 4], \quad\langle\lambda|=\langle 3|+z\langle 4| .
$$

Since $\langle\lambda| \sim z$ and $\mid \lambda] \sim \bar{z}$, we should examine the dependence on $\langle\lambda|$, to find anti-derivative w.r.t. $z$.

Using Eq. (9.122) we make the following simplifications of the various terms in $A$, Eq. (9.121),

$$
\begin{aligned}
& \langle 4 \lambda\rangle=-\langle 34\rangle, \quad\langle 2 \lambda\rangle=\langle 23\rangle+z\langle 24\rangle, \\
& \langle 3 \lambda\rangle=z\langle 34\rangle, \quad\langle\lambda|P| \lambda]=\langle 34\rangle([4 \lambda]-z[3 \lambda]) .
\end{aligned}
$$

Thus Eq. (9.121) becomes

$$
A=-\frac{1}{z\langle 34\rangle} \frac{1}{(z[3 \lambda]-[4 \lambda])^{3}(z\langle 24\rangle+\langle 23\rangle)} .
$$

By partial fractioning, obtaining the primitive with respect to $z$, and dropping the logarithmic terms we get

$$
-\frac{[3 \lambda](2\langle 2|4| \lambda]+\langle 2|3| \lambda])}{\langle 34\rangle[4 \lambda]^{2}\langle 2|3+4| \lambda]^{2}([3 \lambda] z-[4 \lambda])}+\frac{[3 \lambda]}{2\langle 34\rangle[4 \lambda]\langle 2|3+4| \lambda]([3 \lambda] z-[4 \lambda])^{2}} .
$$

We now multiply by the missing factors from Eq. $(9.119)-s_{12}^{3} /(\langle 34\rangle[21]) \times$ $[1 \lambda]^{2} /[2 \lambda]$ and make substitutions from Eq. (9.122)

$$
\begin{aligned}
& {[2 \lambda]=[23]+\bar{z}[24], \quad[3 \lambda]=\bar{z}[34], \quad[4 \lambda]=-[34],} \\
& {[1 \lambda]=-[31]-\bar{z}[41]=-[31]+\bar{z} \frac{\langle 23\rangle[31]}{\langle 24\rangle} .}
\end{aligned}
$$

Further, using momentum conservation we have that $s_{12}=-\langle 34\rangle[34]$. Taking the residue at $\bar{z}=-[23] /[24]$ which is the only pole that gives a contribution and setting $z=-\langle 23\rangle /\langle 24\rangle$ we get

$$
\frac{\langle 34\rangle[23](5\langle 24\rangle[24]+2\langle 23\rangle[23])[31]^{2}}{2\langle 24\rangle[24]^{2}(\langle 24\rangle[24]+\langle 23\rangle[23])[12]} .
$$


Simplifying using momentum conservation, we obtain for the coefficient of the $B_{0}\left(p_{12}, 0,0\right)$ bubble contribution to the amplitude,

$$
\begin{aligned}
\tilde{b}_{0}^{(12)}\left(\bar{q}_{1}^{+}, g_{4}^{-}, g_{3}^{+}, q_{2}^{-}\right) & =-\frac{3}{2} \frac{[23][31]^{2}}{[12][34][24]}+\frac{[23][31]}{[24]^{2}} \\
& \equiv \frac{3}{2} i m_{4}\left(\bar{q}_{1}^{+}, q_{2}^{-}, g_{3}^{+}, g_{4}^{-}\right)-\frac{3}{2} \frac{[31]^{2}}{[41][24]}+\frac{[23][31]}{[24]^{2}}
\end{aligned}
$$

Therefore using Eq. (9.97) the result for $\tilde{b}_{0}^{(23)}$ is

$$
\tilde{b}_{0}^{(23)}\left(\bar{q}_{1}^{+}, g_{4}^{-}, g_{3}^{+}, q_{2}^{-}\right)=\frac{3}{2} \frac{[31]^{2}}{[41][24]}-\frac{[23][31]}{[24]^{2}} \text {. }
$$

The rational part

The rational part can be obtained with a number of methods [30, 79, 122] For this elementary example, the simplest method is to retain the dimensiondependent terms in a Passarino-Veltman decomposition. The result is

$$
\mathcal{R}=\frac{1}{2} \frac{[23][31]^{2}}{[24][34][21]} .
$$

Assembling it all: Inserting the integrals

The result for the lowest order amplitude is, c.f. Eq. (D.35),

$$
-i m_{4}\left(\bar{q}_{1}^{+}, q_{2}^{-}, g_{3}^{+}, g_{4}^{-}\right)=\frac{[31]^{3}}{[34][41][12]} .
$$

The full answer for the color-suppressed primitive amplitude is

$$
\begin{aligned}
& \quad-i \tilde{m}_{4}^{(1)}\left(\bar{q}_{1}^{+}, g_{4}^{-}, g_{3}^{+}, q_{2}^{-}\right)=\frac{1}{2} \frac{[21]^{2}[34]^{2}}{[24]^{3}[41]}\left[s_{12} s_{23} I_{4}\left(0,0,0,0 ; s_{12}, s_{23} ; 0,0,0,0\right)\right. \\
& \left.-\quad 2 s_{12} I_{3}\left(0,0, s_{12} ; 0,0,0\right)-2 s_{23} I_{3}\left(0,0, s_{23} ; 0,0,0\right)\right] \\
& +\quad i m_{4}\left(\bar{q}_{1}, q_{2}, g_{3}, g_{4}\right)\left[s_{12} I_{3}\left(0,0, s_{12} ; 0,0,0\right)+\frac{3}{2} I_{2}\left(s_{12} ; 0,0\right)\right] \\
& +\quad\left(\frac{3}{2} \frac{[31]^{2}}{[41][24]}-\frac{[23][31]}{[24]^{2}}\right)\left[I_{2}\left(s_{23} ; 0,0\right)-I_{2}\left(s_{12} ; 0,0\right)\right] \\
& +\quad \frac{1}{2} \frac{[23][31]^{2}}{[24][34][21]} .
\end{aligned}
$$

The final result for the color suppressed one-loop $q g g \bar{q}$ amplitude, can be obtained by substituting the integrals, Eqs. (E.1,E.3) in Eq. (9.132). The first and third square brackets in Eq. (9.132) are finite in the limit $D \rightarrow 4$. The result assumes a remarkably simple form

$$
\begin{gathered}
\tilde{m}\left(\bar{q}_{1}^{+}, g_{4}^{-}, g_{3}^{+}, q_{2}^{-}\right)=m_{4}\left(\bar{q}_{1}^{+}, q_{2}^{-}, g_{3}^{+}, g_{4}^{-}\right)\left[-\frac{1}{\epsilon^{2}}\left(\frac{\mu^{2}}{-s_{12}}\right)^{\epsilon}-\frac{3}{2 \epsilon}\left(\frac{\mu^{2}}{-s_{12}}\right)^{\epsilon}-\frac{7}{2}\right. \\
\left.-\frac{1}{2} \frac{s_{12}}{s_{13}}\left[\left(1-\frac{s_{12}}{s_{13}} \ln \left(\frac{-s_{23}}{-s_{12}}\right)\right)^{2}+\ln \left(\frac{-s_{23}}{-s_{12}}\right)+\pi^{2} \frac{s_{12}^{2}}{s_{13}^{2}}\right]\right] .
\end{gathered}
$$


After taking account of the different regularization scheme (four-dimensional helicity vs 't Hooft-Veltman) that we are using, this result is in agreement with Ref. [111].

\section{Numerical implementation}

\subsection{Comments on the numerical implementation}

In this Section we describe how $D$-dimensional generalized unitarity can be implemented in a computer code. We focus here only on the implementation of the one-loop contribution to NLO cross-sections. In the spirit of Refs. [123, 124] it is possible to interface the one-loop calculation with a tool that provides the real emission corrections, the subtraction terms, and can carry out the phase space integration. Such an approach has been used recently to compute the NLO QCD corrections to five jet production in $e^{+} e^{-}$annihilation where one-loop amplitudes, calculated with generalized unitarity, were interfaced with Madevent/MadFKS [10].

We would like to walk the reader through the principal steps of the implementation of $D$-dimensional generalized unitarity in a computer code, but without discussing too many technical details. To contrast the numerical implementations of the diagrammatic and unitarity-based calculations, we will first explain how "fully automated" diagrammatic calculations are performed. In order to keep this discussion in line with the main theme of this review, we will assume that the OPP method is employed for the reduction of tensor integrals to the scalar basis, but a Passarino-Veltman reduction could also be used. We note that various implementations of diagrammatic calculations [125, 126, 10, 124] can differ in the details - for example, in how the diagrams are grouped together before the numerical evaluation or in how the rational parts are computed, but are otherwise very similar in spirit.

As the name suggests, diagrammatic calculations are based on Feynman diagrams. The Feynman diagrams for a given process can be automatically generated with programs like QGRAF [59] or FeynArts [60]. To do this, the user needs to specify the allowed vertices and propagators, the incoming and outgoing particles, and the number of loops. Furthermore, the user can efficiently control the diagrams that are generated by these programs and disregard oneparticle reducible diagrams such as tadpole diagrams or self-energy corrections to external lines.

The package QGRAF, for instance, generates all diagrams and produces symbolic output for each of them, listing the vertices and the propagators. The momenta and color flow are automatically assigned to the internal lines using the information on the momenta and color configuration of the external particles. For further computations, the QGRAF output needs to be turned into a form that allows one to specify the "quantum numbers" of the external and internal particles. For example, one needs to provide explicit Feynman rules for the propagators and vertices so that a symbol for the quark propagator with the momentum $p$ and the mass $m$ turns into $i \delta_{a b}(\not p-m)^{-1}$, the quark-gluon 
vertex turns into $-i g_{s} / \sqrt{2} T_{i j}^{a} \gamma_{\mu}$ and so on. It is straightforward to accomplish this using computer algebra programs such as Form [127], Mathematica or Maple. For example, the QGRAF output can be turned into a Form-readable file that contains all Feynman diagrams listed one after the other. The Form program is then executed. The program performs minimal simplifications of the input. It expresses color degrees of freedom through the standardized color basis, contracts and removes all dummy indices to an extent possible, and uses the equations of motion of the external particles to simplify the output. At the end of this process, the Form program writes the contribution of a given Feynman diagram to different color-ordered amplitudes as sums of products of kinematic functions with strings of gamma matrices sandwiched between fermion spinors. The Form program outputs the resulting expression into a file that is further processed by a number of symbolic manipulation routines. Those routines shift the loop momenta in each of the Feynman diagrams, to bring the denominators to a standard form, Eq. (2.6), write out the remaining contractions of dummy indices explicitly and produce Fortran programs that compute numerators and denominators of Feynman diagrams for given value of the loop momentum. In all these manipulations, the number of dimensions is kept as a free parameter that can be passed to the Fortran routines. After these manipulations are done, we are in position to apply the OPP algorithm, as described in Sect. 5.

We now contrast this procedure with the calculation based on generalized unitarity. As a first step, we analytically identify the decomposition of the amplitude for the process of interest into primitive amplitudes. For definiteness, we assume first that the calculation involves only colored particles, in which case all particles in each primitive amplitude are ordered. It follows that there is just one parent diagram for each primitive amplitude and this parent diagram can be described by the momenta, polarizations and flavors of all external particles and flavors of all internal propagators. We note that, for programming purposes, it is often convenient to assume that parent diagrams for amplitudes with $N$ external particles contain $N$ propagators that depend on the loop momenta. However, when fermions are present, some primitive amplitudes contain a smaller number of propagators in their parent diagrams. As we discussed in Sect. 6, to remedy the situation we introduce "dummy lines" [67] which do not correspond to propagators of physical particles and therefore can not be "cut", but which are very convenient for bookkeeping purposes. For example, to describe a contribution to two-quark two-gluon scattering amplitude which is proportional to the number of massless fermions $n_{f}$, we can introduce the parent diagram with one dummy line, while the parent diagram that describes $n_{f}$-dependent contribution to a four-quark amplitude can be drawn using two dummy lines.

When colorless particles are involved, a primitive amplitude may have more than one parent diagram. Those parent diagrams are obtained by considering all possible permutations of the colorless particles with respect to the external quarks and gluons. As an example, consider a gluon-loop correction to the $0 \rightarrow \bar{u} d W^{+} g$ amplitude. While the $W^{+}$-boson is always attached to the fermion line, the gluon can be emitted either by fermions or by the virtual 


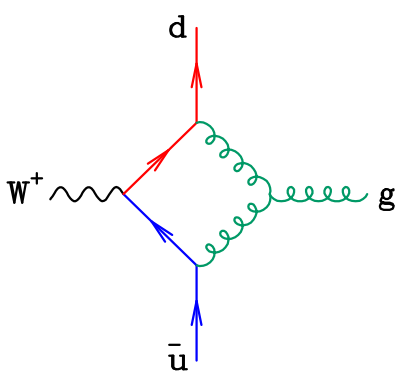

(a)

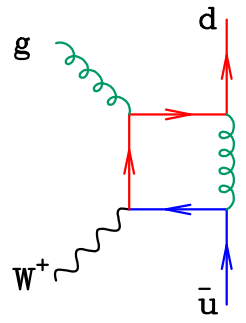

(b)

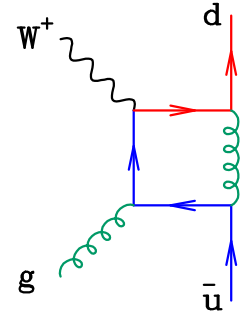

(c)

Figure 10.1: Parent diagrams for the $0 \rightarrow \bar{u} d W^{+} g$ amplitude.

gluon. These two different possibilities correspond to two different primitive amplitudes. When the gluon is emitted by the virtual gluon there is only one possible ordering, $\bar{u} W^{+} d g$, so this primitive amplitude involves only one parent diagram, see Fig. 10.1a. However, when the gluon is emitted from the fermion line, there are two possible orderings of the $W^{+}$-boson with respect to the gluon, $\bar{u} g W^{+} d$ or $\bar{u} W^{+} g d$. As a consequence, this primitive amplitude has two parent diagrams shown in Figs. 10.1b,c.

Following the OPP procedure, we have to consider all possible "cuts" of the parent diagram without cutting dummy lines. This means that we have to identify all possible combinations of the inverse Feynman propagators that can be set to zero simultaneously. The largest member of the set contains five propagators, while the smallest member of the set contains one propagator. In principle, all possible combinations of propagators must be included in this set. However, in practice, sets with single massless propagators or sets with two massless propagators with light-like reference momentum integrate to zero in dimensional regularization and for this reason do not need to be considered.

We study a member of a such a set with $k$ inverse propagators $\left[d_{i_{1}}(l), d_{i_{2}}(l)\right.$, ..,$\left.d_{i_{k}}(l)\right]$. By solving $k$ equations $d_{i_{j}}(l)=0$ with $j=1, \ldots k$, we find the loop momentum $l_{c}$ that satisfies the cut conditions. Since for $l=l_{c}$ the "internal" particles $i_{1}, i_{2}, \ldots i_{k}$ are on-shell and since we know the external particles that must appear between two consecutive internal on-shell particles in the parent diagram, the on-shell scattering amplitude that needs to be calculated is fixed.

In order to calculate the on-shell scattering amplitudes, we employ the Berends-Giele recursion relations explained in Sect. 7.3. We can code up various recursion relations by employing recursive functions, available in Fortran 90. The calculation of various currents can be sped up by avoiding the re-calculation of the currents that have already been computed. This can be done by assigning an integer index given by a different power of two to each external particle. Once this is done, the sum of the indices uniquely specifies a given current for fixed momenta and helicities of the external particles.

We note that amplitudes with multiple fermion pairs or vector bosons are 
complicated and that the knowledge of the quantum numbers of the internal and external particles may be insufficient to fully specify tree-level amplitudes that need to be computed once a particular cut is considered. As an example, consider the case of the four-quark one-loop amplitude with a closed fermion loop. As we explained earlier, the parent diagram involves two dummy lines and two virtual fermion lines. The double cut of the two fermion lines involves two four-quark tree amplitudes. When the flavor of the internal and external quarks is the same, these amplitudes involve $s$ - and $t$-channel contributions. However, one needs to forbid contributions where internal and external quarks couple since these terms do not give rise to a fermion loop contribution. In a computer code, such terms can be easily avoided by always assigning a different flavor to the internal quark lines in amplitudes with closed fermion loops.

As another example we consider the one-loop amplitude for $0 \rightarrow Z q \bar{q} g$. If we deal with color-ordered diagrams and use color-stripped Feynman rules, a diagram exists where the $Z$-boson mixes into an off-shell gluon that splits into quarks and gluons $g^{*} \rightarrow \bar{q} q g$. When color degrees of freedom are restored, such diagram must vanish because of color conservation $\operatorname{Tr}\left(T^{a}\right)=0$. A simple solution to this problem is to introduce special fermion current for internal fermions and to disregard a contribution to this current of the diagram $q \bar{q} \rightarrow$ $g^{*} \rightarrow X$, provided that the invariant mass of the two quarks equals to the mass of the $Z$-boson. These examples illustrate that, occasionally, care is needed in the recursive calculation of the tree-level amplitudes, when they are used as an input for one-loop computations. However, most of the time such calculations are straightforward. We also note that if the OPP reduction is applied to colordressed amplitudes [94] these complications do not arise.

As we explained in Sect. 5, tree on-shell amplitudes are needed to find the OPP reduction coefficients. To determine all the coefficients uniquely, we must deal with spaces of various dimensionalities and consider different embeddings of the loop momenta into those higher-dimensional spaces. The details of these choices are explained in Sect. 5. To make computations efficient, it is important to avoid repeating low-dimensional calculations when higher-dimensional calculations are performed. For example, the $(D-4)$-independent parts of the result are always computed with four-dimensional momenta and polarization vectors. Higher-dimensional calculations are only needed to account for effects of "extra-dimensional" polarizations.

The OPP equations need to be solved once and for all. The actual solutions can be found in a variety of ways. By default, discrete Fourier transforms $[69,70]$ are employed to compute the four-dimensional part, while other procedures, such as e.g. direct Gauss elimination method, are used to calculate reduction coefficients related to the rational parts or the cut-constructible parts in singular kinematics.

\subsection{Checks and numerical instabilities}

When the on-shell/generalized unitarity framework is applied to a new process, new tree-level primitive amplitudes and Berends-Giele currents are in general required. As the first check we verify that when new currents are used with 
four-dimensional momenta and polarization vectors, the tree-level amplitudes coincide with the ones computed with e.g. MadGraph [128]. Furthermore, when coding up new one-loop amplitudes, it is useful to check that coefficients of master integrals scale correctly when momenta and masses of all external particles in the scattering amplitudes are re-scaled by a constant factor. When we move from computing higher-point coefficients to computing lower-point coefficients, we require tree-level amplitudes with higher multiplicity. The re-scaling test allows us to determine exactly the stage in the calculation when something goes wrong which is very useful for debugging purposes.

Of course, the best check of any result is provided by an independent calculation. To this end, we use a Feynman-diagrammatic calculation that employs the OPP method for the reduction of tensor integrals, as described at the beginning of this Section. The Feynman-diagrammatic calculation does not require dealing with primitive amplitudes which provides an important simplification and makes it quite independent from unitarity computations. Since it is sufficient to find agreement between the Feynman-diagrammatic and the unitarity-based calculation for a few selected points, the Feynman-diagrammatic calculation neither needs to be particularly efficient nor does it need to have a rescue system for points that turn out to be numerically unstable.

Typically, calculations are performed in standard double precision. For some kinematic points this may be insufficient if numerical instabilities develop because of divisions by small numbers or because of large cancellations between separate terms. For kinematic points where this happens, it is necessary to repeat the calculation in higher, for example quadruple, precision which is a built-in option in Fortran $90^{18}$ or in multiple precision, with packages like MPLib [129, 130].

However, because higher precision computations are expensive, it is important to have a numerical procedure to detect unstable points, and to repeat the higher-precision calculation for as limited a number of contributions as possible. There are several ways to control the numerical stability of the calculation. For example, one can make use of the fact that divergences of one-loop amplitudes are known [98] (see also Section 6.4). When the numerical reduction is performed and divergent parts of the master integrals are computed with the use of the QCDLoop program [55], the results of the numerical calculation are compared with the predicted [98] values for coefficients of $\epsilon^{-2}$ and $\epsilon^{-1}$ terms. Because two- and one-point functions are divergent, the correct result for the $1 / \epsilon$ pole of the amplitude suggests that the reconstruction of the reduction coefficients works relatively well all the way down to the lowest-point integrals. However, the rational part of the amplitude can not be checked by comparing divergent terms only.

Another way to detect instabilities is the so-called $N=N$ test. Recall that the OPP method works by reconstructing the complete parametric dependence

\footnotetext{
${ }^{18}$ Quadruple precision is not supported by gfortran and g95 compilers but it is supported by the Intel Fortran Compiler (ifort).
} 

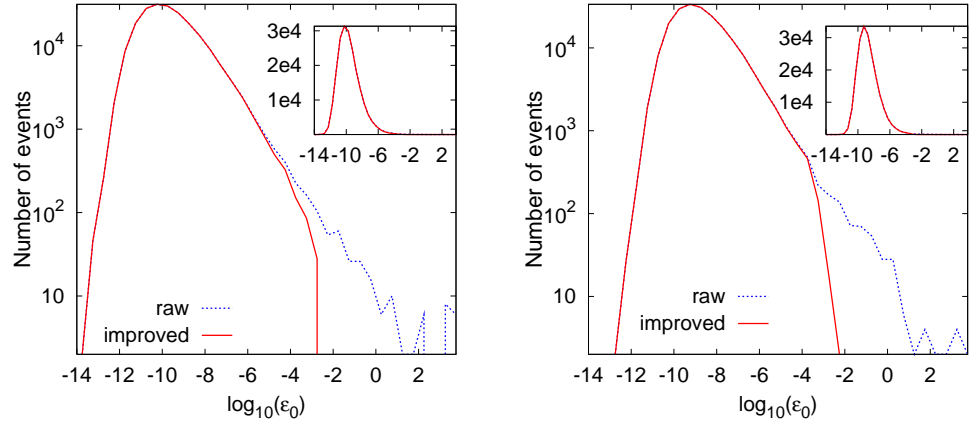

Figure 10.2: Typical improvement in accuracy for some primitive amplitudes that describe $0 \rightarrow W^{+} q \bar{q}+3 g$ process. The accuracy of raw data is shown, in comparison with the results of numerical improvement. The inset shows the same plots in a linear scale. The figures are taken from Ref. [67].

of the integrand on the loop momentum. Therefore, once the OPP coefficients in the decomposition of the one-loop amplitude have been calculated, it is possible, for any arbitrary loop-momentum, to compute a given residue of the amplitude either using the OPP coefficients, or directly as a product of tree-level amplitudes. The two results should coincide up to numerical rounding errors. A larger difference between these two results is a sign of a numerical instability. We note that both, the $(D-4)$ part of the parameterization of the residue of the cut and the $D=4$ part are checked by this test. Moreover, by choosing either four- or five-dimensional momentum on the cut, we can focus the test on either cut-constructible or rational part of a particular amplitude.

However, the $N=N$ test has two unfortunate features. First, since the test needs to be performed for a randomly chosen loop momentum, it may happen that a bad choice of the loop momentum will not detect the numerical instability. Second, as we pointed out in Sect. 5, many of the coefficients in the OPP parameterization vanish after the integration over the directions of respective transverse spaces which implies that the precision with which they are reconstructed is irrelevant for the precision of the final answer. Moreover, even those coefficients that do not vanish under such integration may multiply small or vanishing integrals so that, again, their precision is not important. Hence, we conclude that the $N=N$ test is typically too strong except for unfortunate choices of the test momentum where it can actually overestimate the accuracy of the calculation.

An interesting modification of the $N=N$ test that addresses the shortcomings described above was recently suggested by R. Pittau in Ref. [131]. The idea is to perform the OPP reduction twice, employing different ways to solve systems of linear equations. Since this procedure gives two independent values for the required one-loop amplitude, this is a good check but, if implemented naively, it will simply double the computational time. The trick to avoid this is to use the reconstructed numerator function, rather than the actual one, to 
perform the second OPP reduction. As a result, the increase in the required computational time is modest.

There are other ways to estimate the accuracy of the calculation. For example, in Sect. 5 we explained that the parameterization of the residues involves traceless tensors of restricted rank. This can be checked numerically by performing a Fourier projection on terms that must vanish, to verify that those terms are indeed compatible with zero. This check can be performed at a moderate computational cost. However, the interpretation of the outcome of the test might be difficult, especially when large cancellations between different coefficients take place. Another - rather simple-minded - possibility is to check the size of the one-loop virtual amplitude compared to the Born result. We expect the coefficient of $\alpha_{s} /(2 \pi)$ in the virtual contribution to be of the same order as the tree amplitude. If this is not the case, the phase-space point is considered suspicious and the calculation is repeated in higher precision. In order to find all unstable points, one can combine those tests. The power of these procedures to detect points that are computed incorrectly is shown in Fig. 10.2, taken from Ref. [67]. In a typical computation, less than one percent of all phase-space points used to calculate the virtual corrections, show numerical instabilities and are recomputed with higher precision.

We also note that, instead of recurring to higher precision, one can try to perform a small change in the kinematic configuration so that numerical cancellations that, for the exceptional point, would occur only beyond the double precision happen again in the double precision regime. For instance, one can consider replacing the one-loop result at the exceptional point with the geometric average of the two results obtained using two points in the phase-space that are close enough to the exceptional point [124].

\subsection{Time dependence of the D-dimensional unitarity algorithm}

The development of new techniques for one-loop calculations is mainly driven by the LHC physics, which forces us to study processes with a large number of particles in the final state. If we are to consider processes with higher- and higher multiplicities, it is important to understand how the computational time of the $D$-dimensional unitarity algorithm scales with the number $N$ of external particles.

The calculation of one-loop amplitudes within $D$-dimensional unitarity factorizes into the calculation of several higher-dimensional tree amplitudes. The cost of computing one-loop amplitudes is almost entirely dominated by the time needed for the calculation of these tree-level amplitudes. In order to estimate how the running time scales with the number of external particles involved, it is thus sufficient to count the number of tree-level amplitudes that have to be evaluated and to know how the computational time of these building blocks scales with $N$.

For simplicity, let us first discuss the case of purely gluonic amplitudes. An example involving a quark-pair, a vector boson, and an arbitrary number of gluons will be considered at the end of this Section. As long as only gluons are involved, primitive and color-ordered amplitudes coincide, so that we do 
not need to distinguish between them. Each color-ordered amplitude has only one parent diagram with ordered external gluons. The number of tree-level amplitudes that one needs to compute to determine a single one-loop colorordered amplitude is given by

$$
\begin{aligned}
& n_{\text {tree }}=\left\{\left(D_{s_{1}}-2\right)^{2}+\left(D_{s_{2}}-2\right)^{2}\right\} \\
& \times\left(5 c_{5}\left(\begin{array}{c}
N \\
5
\end{array}\right)+4 c_{4}\left(\begin{array}{c}
N \\
4
\end{array}\right)+3 c_{3}\left(\begin{array}{c}
N \\
3
\end{array}\right)+2 c_{2}^{\prime}\left[\left(\begin{array}{c}
N \\
2
\end{array}\right)-N\right]\right) .
\end{aligned}
$$

The various terms in Eq. (10.1) have the following origin:

- the two terms in the curly brackets take into account the sum over internal polarizations in $D_{s_{1}}$ and $D_{s_{2}}$ dimensions. We note that Eq. (10.1) gives a conservative estimate since it is assumed there that the entire calculation is done in $D_{s_{1}}>4$ and $D_{s_{2}}>4$ dimensions. As already mentioned, it is instead convenient to compute the coefficients needed for the cutconstructible part in $D=4$. This decreases the running time for all $N$-gluon amplitudes by the same amount, so that this does not affect the way the running time scales with $N$;

- the binomial coefficients denote the number of multiple cuts that a oneloop parent diagram, that is composed of massless particles and has $N$ external legs, can have. In the case of double cuts, one can discard the $N$ cuts of self-energies on external legs, therefore one subtracts $N$ from the binomial coefficient;

- the coefficients 5,4,3, and 2 count the number of tree-level amplitudes involved in pentuple, quadruple, triple, and double cuts, respectively;

- finally, the coefficients $c_{i}$ denote the number of independent coefficients in the decomposition of the numerator function. Explicitly, we have $c_{5}=1$, $c_{4}=5, c_{3}=c_{2}=10$. These coefficients also give the number of times one needs to evaluate the numerator function in order to fully solve the OPP system of equations. ${ }^{19}$

Altogether, Eq. (10.1) implies that, for large $N$, we need to evaluate $\propto N^{5}$ treelevel amplitudes, to reconstruct a single one-loop primitive amplitude, for one kinematic point.

As shown in [87] when Berends-Giele recursion relations are implemented in a way that avoids recomputing the same current appearing in different amplitudes, the time needed to compute an $N$-gluon amplitude scales with the number of

\footnotetext{
${ }^{19}$ We note that, for amplitudes with massless internal particles, tadpole diagrams do not need to be calculated. Hence, out of ten OPP coefficients that contribute to each double cut, we need to know just two coefficients that give rise to non-vanishing contributions after integration over the transverse space is performed. This is the reason that $c_{2}^{\prime}=2$ appears in Eq. (10.1).
} 
gluons as

$$
\tau_{\text {tree }}=\left(\begin{array}{c}
N \\
3
\end{array}\right) E_{3}+\left(\begin{array}{c}
N \\
4
\end{array}\right) E_{4} .
$$

In Eq. (10.2), $E_{3,4}$ denote times needed to evaluate a single three- or four-gluon vertex and the binomial coefficients simply count the number of such vertices. Since vertices are never recomputed, their number determines the time needed to compute a tree-level amplitude.

To find the number of three-gluon vertices present in the amplitude described by $N$ ordered gluons with momenta $k_{1}, \ldots, k_{N}$, we note that such amplitude receives a unique contribution from a three-gluon vertex that describes the interaction of three gluon currents with momenta $k_{i_{1}}+k_{i_{1}+1}+\ldots k_{i_{2}}$, $k_{i_{2}+1}+k_{i_{2}+2}+\ldots k_{i_{3}}, k_{i_{3}+1}+k_{i_{3}+2}+\ldots k_{i_{1}-1}$, where all labels need to be understood modulo $N$. Because three numbers $-i_{1}, i_{2}, i_{3}$ - label the three-gluon vertex uniquely, the number of independent three-gluon vertices is given by the number of ways we can choose three integers $i_{1}, i_{2}, i_{3}$ from a set of $N$ numbers. This is exactly the binomial coefficient in the first term of Eq. (10.2). The same reasoning holds for four-gluon vertices as well and explains the binomial coefficient in the second term of Eq. (10.2).

It follows from Eqs. $(10.1,10.2)$ that the overall run-time for one-loop primitive amplitudes scales, for large $N$, as

$$
\tau=\tau_{\text {tree }} \cdot n_{\text {tree }} \sim N^{9}
$$

This is illustrated in Fig. 10.3, taken from Ref. [132] (see also [133, 134]), which shows the time necessary to evaluate tree and one-loop gluon helicity amplitudes as a function of the number of external gluons, as well as a fit to a degree four and a degree nine polynomial.

We close this Section by commenting on what happens in more complicated processes that involve quarks and/or vector bosons. In such cases, for a fixed primitive, Eq. (10.1) needs to be modified to include an additional factor $n_{\text {perm }}$ which counts the number of allowed permutations of colorless particles with respect to quarks and gluons. Eq. (10.2) still holds but $E_{3}$ denotes the average time to compute a three-point vertex, and for four-point vertices $N$ denotes only the number of gluons. For instance, the one-loop amplitude for the process $0 \rightarrow \bar{d} u W^{+}+N$-gluons involves, among others, a leading-color primitive amplitude $A_{v}(\bar{q}, q, g, \ldots, g)$, where all external gluons are emitted from the gluon in the loop, and a sub-leading color primitive amplitude $A_{v}(\bar{q}, g, \ldots g, q)$, where all gluons are emitted from the quark line. In the case of the leading-color amplitude, no permutation of the $W$ boson is allowed, so that $n_{\text {perm }}=1$. For $N=3$ the corresponding parent diagram is shown in Fig. 10.4a. In contrast, in the case of the sub-leading-color amplitude there are $n_{\text {perm }}=n-2$ possible attachments of the $W$ on the quark-line, where $n$ denotes the total number of particles, i.e., $n=N+3$. For $N=3$ this primitive has four different parent diagrams, as illustrated in Figs. 10.4b-e.

Fig. 10.5 shows the running time for the leading-color amplitude, the subleading color one, as well as the latter one rescaled by a factor of $n_{\text {perm }}=n-2$. 


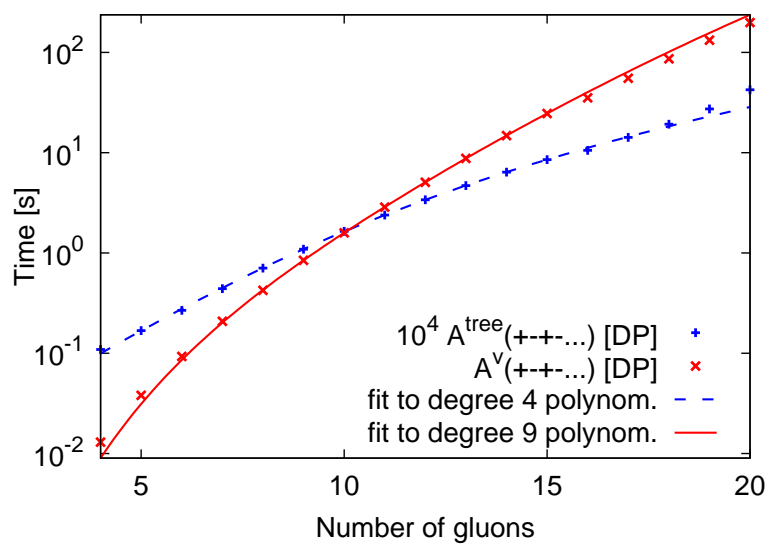

Figure 10.3: Time in seconds needed to compute tree amplitudes (dashed (blue online)) and one-loop ordered amplitudes (solid (red online)) with gluons of alternating helicity signs, $A_{N}^{[1]}(+-+-+\ldots)$, as a function of the number of external gluons ranging between 4 to 20 . The notation $[\mathrm{DP}]$ refers to numerical computations performed with double precision accuracy in Fortran. Source: Figure taken from Ref. [132].

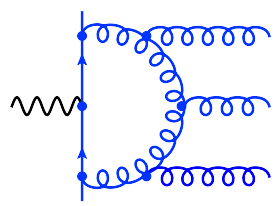

(a)

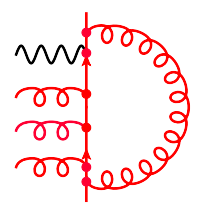

(b)

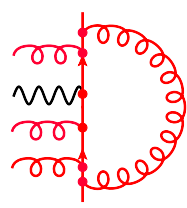

(c)

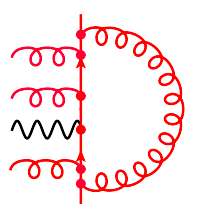

(d)

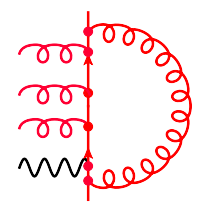

(e)

Figure 10.4: a) Parent diagram for the leading-color primitive amplitude $A_{v}\left(\bar{q}, q, g_{1}, g_{2}, g_{3}\right)$; b-e) Parent diagrams for the sub-leading-color primitive amplitude $A_{v}\left(\bar{q}, g_{1}, g_{2}, g_{3}, q\right)$.

The running time for both amplitudes is a polynomial in $n$ but, as expected, the sub-leading color amplitude is $(n-2)$ times slower than the leading color one. Using the numerical dominance of the leading-color amplitudes, one can organize the calculation in such a way that contributions of sub-leading color amplitudes to cross-sections are computed with the smaller relative accuracy than leading-color amplitudes. Such organization of the calculation ensures that not too much time is spent on the calculation of pieces of the amplitude that give small contributions to the final answer. These ideas have been used in recent NLO calculation of some complicated processes [7, 17].

\section{Outlook}

Perturbation theory plays a primary role in phenomenological applications of quantum field theory. The first non-trivial step in the perturbative expansion requires dealing with tree processes; this is well-understood by now. Indeed, 


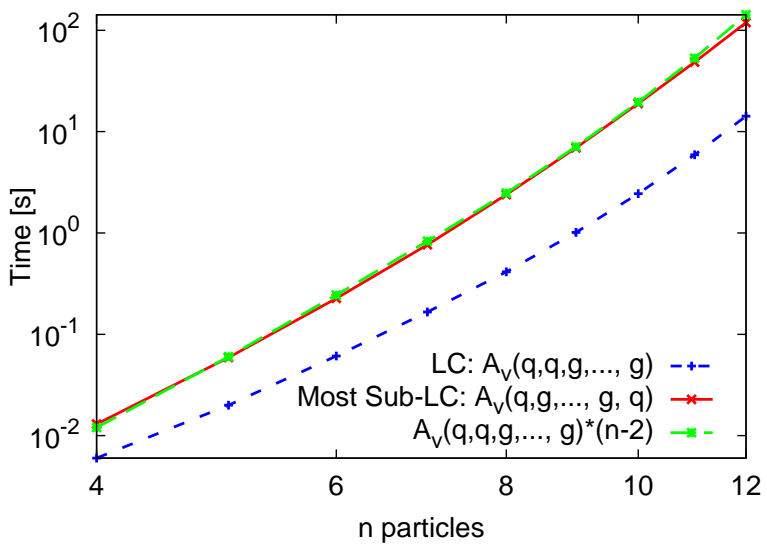

Figure 10.5: Time in seconds needed to compute the leading-color (LC) primitive amplitude $A_{v}(q, q, g \ldots g)$ and the sub-leading-color primitive amplitude $A_{v}(q, g \ldots g, q)$ for the process $0 \rightarrow \bar{u} d W^{+}(n-3) g$, as a function of the total number of particles $n$. The ratio of times required to compute sub-leading-color and leading color is given by $n-2$, with a very good approximation, so that it is hard to distinguish the solid and the dashed lines.

there are several automated computer programs $[135,136,128,137]$ that can, at leading order, calculate any process in the Standard Model and its most popular extensions. Given the scope of the LHC physics program and the benefits of improving the reliability of theoretical predictions, automating one-loop computations is considered to be an important goal in phenomenologically-oriented high-energy theory and it took about ten years of active research of many physicists to get close to accomplishing this goal. Interestingly, arriving at this point required achieving a deeper understanding of perturbation theory in general and resulted in the development of a new framework for perturbative computations in quantum field theory that goes beyond Feynman diagrams and traditional methods of Passarino-Veltman reduction.

The subject of this review - numerical $D$-dimensional unitarity - is one of the results of multiple attempts, undertaken in recent years by many theorists, to provide a flexible enough framework to serve as a basis for automation. Conceptually, $D$-dimensional unitarity is very different from Feynman diagrams. It is an $S$-matrix approach where locality is not manifest and loop amplitudes are bootstrapped from tree amplitudes using unitarity. The tree-level scattering amplitudes need to be known for complex on-shell momenta and space-times of higher dimensionality. As we explained in this review, analytic continuation of on-shell scattering amplitudes to higher-dimensional space-times is easily accomplished by using Berends-Giele recursion relations to construct tree amplitudes. Moreover, polarization states of bosons and fermions can be extended to higherdimensional space times in a straightforward way if one does not require using spinor-helicity methods in those calculations.

Generalized $D$-dimensional unitarity provides full amplitudes and works for 
massless and massive particles, providing the level of robustness that is required to address problems of high phenomenological interest. In this review, we tried to emphasize this robustness by avoiding the spinor-helicity formalism in the decomposition of the loop momenta entering the unitarity cuts. An intuitively simple decomposition of the loop momentum into physical and transverse spaces that identifies very clearly the degrees of freedom that are left unconstrained by unitarity cuts and a geometrical picture of the transverse space allow us to choose a parametrization of the transverse space in a most suitable and simple way. We believe that, in doing so, many subtle features of the unitarity-based approaches to one-loop computations are made very transparent.

Before closing, we mention important research topics that we have not discussed in this review. First, we only briefly mentioned the recent developments in Passarino-Veltman reduction technology [138, 63, 119, 61] that lead to efficient and numerically-stable ways of computing multi-leg one-loop Feynman diagrams $[2,12]$. While these methods are traditional, the results that have been achieved by applying them to realistic problems are spectacular and the potential of traditional computational methods does not seem to be anywhere near to being exhausted.

Second, in this review we did not talk about attempts to design a framework where one-loop computations can be performed by pure numerical methods $[33,34,35,36,37,38,39,40,44,41,42,43,44,139]$. It will be interesting to see how numerical methods will develop in the future, given the robust seminumerical nature of the OPP approach and recent attempts to automate it $[17,124]$.

Third, we only briefly discussed the spinor-helicity methods [140] that play an important role in analytical computations of tree amplitudes. In particular, those methods tend to provide very compact expressions for tree amplitudes when external particles are in definite helicity states. Since tree amplitudes play an important role in unitarity-based computations, one may expect that spinorhelicity methods may be useful for boosting the efficiency of one-loop computations of scattering amplitudes $[141,142]$. Moreover, spinor-helicity methods can be used to provide compact expressions for one-loop scattering amplitudes as well, see e.g Refs. $[84,19,143,144,106]$. At the moment, it seems that the benefits of analytic computations lie more in clarifying the generic properties of the amplitudes, rather than in their phenomenological applications. In particular, it is unclear if the benefits gained in the computing time that follow from using analytic formulas in numerical codes are sufficiently high, to outweigh the significant human efforts that are required to obtain those analytic results.

Fourth, the ideas of unitarity overlap, at least partially, with many exciting new developments in the field of computation of scattering amplitudes in supersymmetric extensions of QCD and, in particular, $\mathcal{N}=4$ SUSY Yang-Mills. It is an interesting and non-trivial question to what extent real needs of the physics program at the LHC can benefit from those developments. While it appears that many of the developments in that field rely on high degree of supersymmetry, a number of ideas such as the recursion relations for the loop integrand $[145,146,147]$ may have more general nature and, perhaps, can be successfully 
used in non-supersymmetric quantum field theories, such as QCD and the Standard Model. Partially related to this is the barely explored question of whether it is possible to apply unitarity-based ideas to multi-loop computations. On one hand, we do know that this is possible in highly-supersymmetric theories, where calculations are carried out to a very high order in the perturbative expansion. On the other hand, in non-supersymmetric field theories, even the first step the identification of a suitable basis of master integrals at two-loops - has been attempted only recently [148]. Hence, it will be interesting to watch in the future how new ideas developed in the context of $\mathcal{N}=4$ SUSY Yang-Mills will penetrate into more phenomenological research related to perturbative computations in the Standard Model.

The field of perturbative computations for multi-particle processes went through a remarkable transformation in the past few years. During these years, the ability to perform specific computations that are of importance for the Tevatron and the LHC physics program has increased beyond the most optimistic expectations. The improvement in our understanding of perturbative quantum field theory - that is a byproduct of these exciting developments - gives us hope that the momentum of the past several years can be carried forward, so that even more complicated physics - both in terms of the number of external particles and in terms of the number of loops - can be addressed.

\section{Acknowledgements}

We would like to thank W. Giele, T. Melia, M. Schulze, R. Röntsch for collaboration on various topics that we address in this review. We are grateful to F. Caola, W. Giele and M. Schulze for comments on the manuscript. Z.K. would like to thank the Institute for the Physics and Mathematics of the Universe (IPMU), University of Tokyo, Kashiwa, Japan, and the Department for Theoretical Physics and the Cosmos, University of Granada, Spain for hospitality where parts of this review have been prepared. This research is supported by the NSF under grant PHY-0855365, by the British Science and Technology Council, by the LHCPhenoNet network under the Grant Agreement PITN-GA2010-264564, and by the European Research and Training Network (RTN) grant Unification in the LHC ERA under the Agreement PITN-GA-2009-237920. Fermilab is operated by Fermi Research Alliance, LLC under Contract No. DEAC02-07CH11359 with the United States Department of Energy.

\section{Appendix A. Details of Passarino-Veltman decomposition}

The purpose of this appendix is to give a full description of the PassarinoVeltman decomposition [58] for the bubble-, triangle-, and box- tensor integrals which appear in a renormalizable theory. Although these formulas have been given in the original paper it may be of use to present them here. In presenting them we have converted the reduction formula to the Bjorken and Drell metric $[149] g_{\mu \nu}=\operatorname{diag}[1,-1,-1,-1]$, which is most commonly used. In addition, 
we believe that we have improved the notation so that the pattern of the reduction is more apparent. Furthermore the original published paper contains typographical errors (on line 2 and line 17 of page 205 of ref. [58]). We believe that the above remarks provide sufficient justification for presenting the Passarino-Veltman reduction formulas again.

\section{Appendix A.1. Two-point functions}

We first recall (cf. Section 2) our definitions of the form-factor expansions for the tensor integrals

$$
\begin{aligned}
B^{\mu} & =p_{1}^{\mu} B_{1}, \\
B^{\mu \nu} & =g^{\mu \nu} B_{00}+p_{1}^{\mu} p_{1}^{\nu} B_{11} .
\end{aligned}
$$

We shall refer to the coefficients $B_{i}, B_{00}, B_{11}$ as form factors. The dependence of these form factors on the appropriate Lorentz invariants, such as squares of the external momenta and masses of internal lines has been dropped for brevity.

By contracting through with $p_{1}$ and $g^{\mu \nu}$ the form factors can be expressed entirely in terms of scalar integrals. (The singular case $p_{1}^{2}=0$ needs to be handled separately). The results are,

$$
\begin{aligned}
B_{1} & =\frac{1}{2 p_{1}^{2}}\left(f_{1} B_{0}(1,2)+A_{0}(1)-A_{0}(2)\right), \\
B_{11} & =\frac{1}{2 p_{1}^{2}}\left(f_{1} B_{1}(1,2)+A_{0}(2)-2 B_{00}(1,2)\right), \\
B_{00} & =\frac{1}{2(D-1)}\left(2 m_{1}^{2} B_{0}(1,2)+A_{0}(2)-f_{1} B_{1}(1,2)\right) .
\end{aligned}
$$

The compact notation is as follows. The propagators that occur in the scalar integrals, $A_{0}$ and $B_{0}$ are specified by $i$ where $m_{i}$ is the mass which is present in the propagator. Thus, for instance,

$$
A_{0}(2)=\frac{1}{i \pi^{D / 2}} \int d^{D} l \frac{1}{l^{2}-m_{2}^{2}} .
$$

The constant $f_{1}$ introduced in Eq. (A.3) is part of the loop-momentum independent offsets that we will use in what follows

$$
\begin{aligned}
& f_{1}=m_{2}^{2}-m_{1}^{2}-p_{1}^{2}, \\
& f_{2}=m_{3}^{2}-m_{2}^{2}-p_{2}^{2}-2 p_{1} \cdot p_{2}, \\
& f_{3}=m_{4}^{2}-m_{3}^{2}-p_{3}^{2}-2 p_{3} \cdot p_{2}-2 p_{3} \cdot p_{1} .
\end{aligned}
$$




\begin{tabular}{|cc|c|}
\hline \multicolumn{2}{|c|}{ Form factors } & RHS \\
\hline$C_{1}$ & $C_{2}$ & $R^{[c]}$ \\
\hline$C_{11}$ & $C_{12}$ & $R^{[c 1]}$ \\
$C_{12}$ & $C_{22}$ & $R^{[c 2]}$ \\
\hline$C_{001}$ & $C_{002}$ & $R^{[c 00]}$ \\
$C_{111}$ & $C_{112}$ & $R^{[c 11]}$ \\
$C_{112}$ & $C_{122}$ & $R^{[c 12]}$ \\
$C_{122}$ & $C_{222}$ & $R^{[c 22]}$ \\
\hline
\end{tabular}

Table A.1: Pairs of form factors which satisfy equations of the form Eq. (A.9).

\section{Appendix A.2. Three-point functions}

Turning now to the triangle integrals we have the form factor expansion

$$
\begin{aligned}
C^{\mu} & =p_{1}^{\mu} C_{1}+p_{2}^{\mu} C_{2}, \\
C^{\mu \nu} & =g^{\mu \nu} C_{00}+p_{1}^{\mu} p_{1}^{\nu} C_{11}+p_{2}^{\mu} p_{2}^{\nu} C_{22}+\left(p_{1}^{\mu} p_{2}^{\nu}+p_{2}^{\mu} p_{1}^{\nu}\right) C_{12} \\
& =g^{\mu \nu} C_{00}+\sum_{i, j=1}^{2} p_{i}^{\mu} p_{j}^{\nu} C_{i j}, \text { where } C_{21}=C_{12} \\
C^{\mu \nu \alpha} & =\sum_{i=1}^{2}\left(g^{\mu \nu} p_{i}^{\alpha}+g^{\nu \alpha} p_{i}^{\mu}+g^{\alpha \mu} p_{i}^{\nu}\right) C_{00 i}+\sum_{i, j, k=1}^{2} p_{i}^{\mu} p_{j}^{\nu} p_{k}^{\alpha} C_{i j k} .
\end{aligned}
$$

These too can be reduced to scalar integrals by contracting through with external momenta and the metric tensor as explained in Section 2.3. For example, for the two form factors $C_{1}, C_{2}$ we have

$$
\left(\begin{array}{c}
C_{1} \\
C_{2}
\end{array}\right)=G_{2}^{-1}\left(\begin{array}{c}
R_{1}^{[c]} \\
R_{2}^{[c]}
\end{array}\right),
$$

where $G_{2}$ is the $2 \times 2$ Gram matrix

$$
G_{2}=\left(\begin{array}{ll}
p_{1} \cdot p_{1} & p_{1} \cdot p_{2} \\
p_{1} \cdot p_{2} & p_{2} \cdot p_{2}
\end{array}\right)
$$

All the form factor pairs which satisfy equations of the form of Eq. (A.9) are shown in Table A.1. Note that three of the coefficients in Table A.1 can be determined in more than one way. This provides an important check of the reduction. The expressions for the right-hand sides listed in Table A.1 are,

$$
\begin{aligned}
& R_{1}^{[c]}=\frac{1}{2}\left(f_{1} C_{0}(1,2,3)+B_{0}(1,3)-B_{0}(2,3)\right), \\
& R_{2}^{[c]}=\frac{1}{2}\left(f_{2} C_{0}(1,2,3)+B_{0}(1,2)-B_{0}(1,3)\right) . \\
& R_{1}^{[c 1]}=\frac{1}{2}\left(f_{1} C_{1}(1,2,3)+B_{1}(1,3)+B_{0}(2,3)-2 C_{00}(1,2,3)\right), \\
& R_{2}^{[c 1]}=\frac{1}{2}\left(f_{2} C_{1}(1,2,3)+B_{1}(1,2)-B_{1}(1,3)\right),
\end{aligned}
$$




$$
\begin{aligned}
& R_{1}^{[c 2]}=\frac{1}{2}\left(f_{1} C_{2}(1,2,3)+B_{1}(1,3)-B_{1}(2,3)\right), \\
& R_{2}^{[c 2]}=\frac{1}{2}\left(f_{2} C_{2}(1,2,3)-B_{1}(1,3)-2 C_{00}(1,2,3)\right), \\
& R_{1}^{[c 11]}=\frac{1}{2}\left(f_{1} C_{11}(1,2,3)+B_{11}(1,3)-B_{0}(2,3)-4 C_{001}(1,2,3)\right), \\
& R_{2}^{[c 11]}=\frac{1}{2}\left(f_{2} C_{11}(1,2,3)+B_{11}(1,2)-B_{11}(1,3)\right), \\
& R_{1}^{[c 22]}=\frac{1}{2}\left(f_{1} C_{22}(1,2,3)+B_{11}(1,3)-B_{11}(2,3)\right), \\
& R_{2}^{[c 22]}=\frac{1}{2}\left(f_{2} C_{22}(1,2,3)-B_{11}(1,3)-4 C_{002}(1,2,3)\right), \\
& R_{1}^{[c 12]}=\frac{1}{2}\left(f_{1} C_{12}(1,2,3)+B_{11}(1,3)+B_{1}(2,3)-2 C_{002}(1,2,3)\right), \\
& R_{2}^{[c 12]}=\frac{1}{2}\left(f_{2} C_{12}(1,2,3)-B_{11}(1,3)-2 C_{001}(1,2,3)\right), \\
& R_{1}^{[c 00]}=\frac{1}{2}\left(f_{1} C_{00}(1,2,3)+B_{00}(1,3)-B_{00}(2,3)\right), \\
& R_{2}^{[c 00]}=\frac{1}{2}\left(f_{2} C_{00}(1,2,3)+B_{00}(1,2)-B_{00}(1,3)\right) .
\end{aligned}
$$

To express our results we have introduced a compact notation. Thus, for example, in Eq. (A.15), $B_{11}(2,3)$ is defined as the $B_{11}$ coefficient of the integral

$$
B^{\mu \nu}\left(p_{2}, m_{2}, m_{3}\right)=\frac{1}{i \pi^{D / 2}} \int d^{D} l \frac{l^{\mu} l^{\nu}}{\left(l^{2}-m_{2}^{2}\right)\left(\left(l+p_{2}\right)^{2}-m_{3}^{2}\right)} .
$$

Note that in Eq. (A.18) the loop momentum $l$ has been shifted $\left(l \rightarrow l-p_{1}\right)$ with respect to the defining equation for the triangle integrals,

$$
\begin{aligned}
& C_{0}\left(p_{1}, p_{2}, m_{1}, m_{2}, m_{3}\right)= \\
& \frac{1}{i \pi^{D / 2}} \int d^{D} l \frac{1}{\left(l^{2}-m_{1}^{2}\right)\left(\left(l+p_{1}\right)^{2}-m_{2}^{2}\right)\left(\left(l+p_{1}+p_{2}\right)^{2}-m_{3}^{2}\right)} .
\end{aligned}
$$

In addition we have the following relations,

$$
\begin{aligned}
C_{00}(1,2,3) & =\frac{1}{2(D-2)}\left(2 m_{1}^{2} C_{0}(1,2,3)-f_{1} C_{1}(1,2,3)-f_{2} C_{2}(1,2,3)\right. \\
& \left.+B_{0}(2,3)\right), \\
C_{001}(1,2,3) & =\frac{1}{2(D-1)}\left(2 m_{1}^{2} C_{1}(1,2,3)-f_{1} C_{11}(1,2,3)-f_{2} C_{12}(1,2,3)\right. \\
& \left.-B_{0}(2,3)\right), \\
C_{002}(1,2,3) & =\frac{1}{2(D-1)}\left(2 m_{1}^{2} C_{2}(1,2,3)-f_{1} C_{12}(1,2,3)-f_{2} C_{22}(1,2,3)\right. \\
& \left.+B_{1}(2,3)\right) .
\end{aligned}
$$


Note that Eqs. (A.20,A.21,A.22) are the only places where the dependence on the dimensionality of space-time $D$ appears. Furthermore $C_{001}, C_{002}$ are also determined by $D$-independent relations of the form of Eq. (A.9) as shown in Table A.1.

\section{Appendix A.3. Four-point functions}

Turning now to the box coefficients we have the form factor expansion,

$$
\begin{aligned}
D^{\mu} & =p_{1}^{\mu} D_{1}+p_{2}^{\mu} D_{2}+p_{3}^{\mu} D_{3}, \\
D^{\mu \nu} & =g^{\mu \nu} D_{00}+\sum_{i, j=1}^{3} p_{i}^{\mu} p_{j}^{\nu} D_{i j}, \\
D^{\mu \nu \alpha} & =\sum_{i=1}^{3} g^{\{\mu \nu} p_{i}^{\alpha\}} D_{00 i}+\sum_{i, j, k=1}^{3} p_{i}^{\mu} p_{j}^{\nu} p_{k}^{\alpha} D_{i j k}, \\
D^{\mu \nu \alpha \beta} & =g^{\{\mu \nu} g^{\alpha \beta\}} D_{0000}+\sum_{i, j=1}^{3} g^{\{\mu \nu} p_{l}^{\alpha} p_{j}^{\beta\}} D_{00 i j} \\
& +\sum_{i, j, k, l=1}^{3} p_{i}^{\mu} p_{j}^{\nu} p_{k}^{\alpha} p_{l}^{\beta} D_{i j k l} .
\end{aligned}
$$

The curly braces denote full symmetrization of the indices. Many of these coefficients satisfy equations of the form

$$
\left(\begin{array}{c}
D_{1} \\
D_{2} \\
D_{3}
\end{array}\right)=G_{3}^{-1}\left(\begin{array}{c}
R_{1}^{[d]} \\
R_{2}^{[d]} \\
R_{3}^{[d]}
\end{array}\right),
$$

where $G_{3}$ is the $3 \times 3$ Gram matrix

$$
G_{3}=\left(\begin{array}{ccc}
p_{1} \cdot p_{1} & p_{1} \cdot p_{2} & p_{1} \cdot p_{3} \\
p_{1} \cdot p_{2} & p_{2} \cdot p_{2} & p_{2} \cdot p_{3} \\
p_{3} \cdot p_{1} & p_{3} \cdot p_{2} & p_{3} \cdot p_{3}
\end{array}\right) .
$$

All the form factor triplets which satisfy equations of the form Eq. (A.27) are shown in Table A.2, where the notation for the right-hand sides of the corresponding linear equation is also defined.

We now give the definitions of the functions $R$ for the rank one boxes,

$$
\begin{aligned}
& R_{1}^{[d]}=\frac{1}{2}\left(f_{1} D_{0}(1,2,3,4)+C_{0}(1,3,4)-C_{0}(2,3,4)\right), \\
& R_{2}^{[d]}=\frac{1}{2}\left(f_{2} D_{0}(1,2,3,4)+C_{0}(1,2,4)-C_{0}(1,3,4)\right), \\
& R_{3}^{[d]}=\frac{1}{2}\left(f_{3} D_{0}(1,2,3,4)+C_{0}(1,2,3)-C_{0}(1,2,4)\right) .
\end{aligned}
$$




\begin{tabular}{|ccc|c|}
\hline \multicolumn{3}{|c|}{ Form factors } & RHS \\
\hline$D_{1}$ & $D_{2}$ & $D_{3}$ & $R^{[d]}$ \\
\hline$D_{11}$ & $D_{12}$ & $D_{13}$ & $R^{[d 1]}$ \\
$D_{12}$ & $D_{22}$ & $D_{23}$ & $R^{[d 2]}$ \\
$D_{13}$ & $D_{23}$ & $D_{33}$ & $R^{[d 3]}$ \\
\hline$D_{001}$ & $D_{002}$ & $D_{003}$ & $R^{[d 00]}$ \\
$D_{112}$ & $D_{122}$ & $D_{123}$ & $R^{[d 12]}$ \\
$D_{113}$ & $D_{123}$ & $D_{133}$ & $R^{[d 13]}$ \\
$D_{123}$ & $D_{223}$ & $D_{233}$ & $R^{[d 23]}$ \\
$D_{111}$ & $D_{112}$ & $D_{113}$ & $R^{[d 11]}$ \\
$D_{122}$ & $D_{222}$ & $D_{223}$ & $R^{[d 22]}$ \\
$D_{133}$ & $D_{233}$ & $D_{333}$ & $R^{[d 33]}$ \\
\hline$D_{0011}$ & $D_{0012}$ & $D_{0013}$ & $R^{d 001]}$ \\
$D_{0012}$ & $D_{0022}$ & $D_{0023}$ & $R^{[d 002]}$ \\
$D_{0013}$ & $D_{0023}$ & $D_{0033}$ & $R^{[d 003]}$ \\
$D_{1111}$ & $D_{1112}$ & $D_{1113}$ & $R^{[d 111]}$ \\
$D_{1222}$ & $D_{2222}$ & $D_{2223}$ & $R^{[d 222]}$ \\
$D_{1333}$ & $D_{2333}$ & $D_{3333}$ & $R^{[d 333]}$ \\
$D_{1112}$ & $D_{1122}$ & $D_{1123}$ & $R^{[d 112]}$ \\
$D_{1113}$ & $D_{1123}$ & $D_{1133}$ & $R^{[d 113]}$ \\
$D_{1122}$ & $D_{1222}$ & $D_{1223}$ & $R^{[d 122]}$ \\
$D_{1133}$ & $D_{1233}$ & $D_{1333}$ & $R^{[d 133]}$ \\
$D_{1223}$ & $D_{2223}$ & $D_{2233}$ & $R^{[d 223]}$ \\
$D_{1233}$ & $D_{2233}$ & $D_{2333}$ & $R^{[d 233]}$ \\
$D_{1123}$ & $D_{1223}$ & $D_{1233}$ & $R^{[d 123]}$ \\
\hline
\end{tabular}

Table A.2: Triplets of form factors which satisfy equations of the form, Eq. (A.27).

For the rank two boxes we obtain,

$$
\begin{aligned}
R_{1}^{[d 1]} & =\frac{1}{2}\left(f_{1} D_{1}(1,2,3,4)+C_{0}(2,3,4)+C_{1}(1,3,4)-2 D_{00}(1,2,3,4)\right), \\
R_{2}^{[d 1]} & =\frac{1}{2}\left(f_{2} D_{1}(1,2,3,4)-C_{1}(1,3,4)+C_{1}(1,2,4)\right), \\
R_{3}^{[d 1]} & =\frac{1}{2}\left(f_{3} D_{1}(1,2,3,4)+C_{1}(1,2,3)-C_{1}(1,2,4)\right), \\
R_{1}^{[d 2]} & =\frac{1}{2}\left(f_{1} D_{2}(1,2,3,4)-C_{1}(2,3,4)+C_{1}(1,3,4)\right), \\
R_{2}^{[d 2]} & =\frac{1}{2}\left(f_{2} D_{2}(1,2,3,4)-C_{1}(1,3,4)+C_{2}(1,2,4)-2 D_{00}(1,2,3,4)\right), \\
R_{3}^{[d 2]} & =\frac{1}{2}\left(f_{3} D_{2}(1,2,3,4)+C_{2}(1,2,3)-C_{2}(1,2,4)\right), \\
R_{1}^{[d 3]} & =\frac{1}{2}\left(f_{1} D_{3}(1,2,3,4)-C_{2}(2,3,4)+C_{2}(1,3,4)\right), \\
R_{2}^{[d 3]} & =\frac{1}{2}\left(f_{2} D_{3}(1,2,3,4)-C_{2}(1,3,4)+C_{2}(1,2,4)\right), \\
R_{3}^{[d 3]} & =\frac{1}{2}\left(f_{3} D_{3}(1,2,3,4)-C_{2}\left(1,2,4522 D_{00}(1,2,3,4)\right) .\right.
\end{aligned}
$$


For the rank three boxes we have,

$$
\begin{aligned}
& R_{1}^{[d 12]}=\frac{1}{2}\left(f_{1} D_{12}(1,2,3,4)+C_{1}(2,3,4)+C_{11}(1,3,4)-2 D_{002}(1,2,3,4)\right), \\
& R_{2}^{[d 12]}=\frac{1}{2}\left(f_{2} D_{12}(1,2,3,4)+C_{12}(1,2,4)-C_{11}(1,3,4)-2 D_{001}(1,2,3,4)\right), \\
& R_{3}^{[d 12]}=\frac{1}{2}\left(f_{3} D_{12}(1,2,3,4)+C_{12}(1,2,3)-C_{12}(1,2,4)\right) . \\
& R_{1}^{[d 13]}=\frac{1}{2}\left(f_{1} D_{13}(1,2,3,4)+C_{2}(2,3,4)+C_{12}(1,3,4)-2 D_{003}(1,2,3,4)\right), \\
& R_{2}^{[d 13]}=\frac{1}{2}\left(f_{2} D_{13}(1,2,3,4)-C_{12}(1,3,4)+C_{12}(1,2,4)\right), \\
& R_{3}^{[d 13]}=\frac{1}{2}\left(f_{3} D_{13}(1,2,3,4)-C_{12}(1,2,4)-2 D_{001}(1,2,3,4)\right) . \\
& R_{1}^{[d 23]}=\frac{1}{2}\left(f_{1} D_{23}(1,2,3,4)-C_{12}(2,3,4)+C_{12}(1,3,4)\right), \\
& R_{2}^{[d 23]}=\frac{1}{2}\left(f_{2} D_{23}(1,2,3,4)+C_{22}(1,2,4)-C_{12}(1,3,4)-2 D_{003}(1,2,3,4)\right), \\
& R_{3}^{[d 23]}=\frac{1}{2}\left(f_{3} D_{23}(1,2,3,4)-C_{22}(1,2,4)-2 D_{002}(1,2,3,4)\right) . \\
& R_{1}^{[d 11]}=\frac{1}{2}\left(f_{1} D_{11}(1,2,3,4)-C_{0}(2,3,4)+C_{11}(1,3,4)-4 D_{001}(1,2,3,4)\right), \\
& R_{2}^{[d 11]}=\frac{1}{2}\left(f_{2} D_{11}(1,2,3,4)-C_{11}(1,3,4)+C_{11}(1,2,4)\right), \\
& R_{3}^{[d 11]}=\frac{1}{2}\left(f_{3} D_{11}(1,2,3,4)+C_{11}(1,2,3)-C_{11}(1,2,4)\right) . \\
& R_{1}^{[d 22]}=\frac{1}{2}\left(f_{1} D_{22}(1,2,3,4)-C_{11}(2,3,4)+C_{11}(1,3,4)\right), \\
& R_{2}^{[d 22]}=\frac{1}{2}\left(f_{2} D_{22}(1,2,3,4)-C_{11}(1,3,4)+C_{22}(1,2,4)-4 D_{002}(1,2,3,4)\right) \text {, } \\
& R_{3}^{[d 22]}=\frac{1}{2}\left(f_{3} D_{22}(1,2,3,4)+C_{22}(1,2,3)-C_{22}(1,2,4)\right) . \\
& R_{1}^{[d 33]}=\frac{1}{2}\left(f_{1} D_{33}(1,2,3,4)-C_{22}(2,3,4)+C_{22}(1,3,4)\right), \\
& R_{2}^{[d 33]}=\frac{1}{2}\left(f_{2} D_{33}(1,2,3,4)-C_{22}(1,3,4)+C_{22}(1,2,4)\right), \\
& R_{3}^{[d 33]}=\frac{1}{2}\left(f_{3} D_{33}(1,2,3,4)-C_{22}(1,2,4)-4 D_{003}(1,2,3,4)\right) .
\end{aligned}
$$




$$
\begin{aligned}
R_{1}^{[d 00]} & \left.=\frac{1}{2}\left(f_{1} D_{00}(1,2,3,4)-C_{00}(2,3,4)+C_{00}, 1,3,4\right)\right), \\
R_{2}^{[d 00]} & \left.\left.=\frac{1}{2}\left(f_{2} D_{00}(1,2,3,4)-C_{00}, 1,3,4\right)+C_{00}, 1,2,4\right)\right), \\
R_{3}^{[d 00]} & \left.=\frac{1}{2}\left(f_{3} D_{00}(1,2,3,4)+C_{00}(1,2,3)-C_{00}, 1,2,4\right)\right) .
\end{aligned}
$$

For the rank four boxes we obtain

$$
\begin{aligned}
& R_{1}^{[d 111]}=\frac{1}{2}\left(f_{1} D_{111}(1,2,3,4)+C_{111}(1,3,4)+C_{0}(2,3,4)-6 D_{0011}(1,2,3,4)\right), \\
& R_{2}^{[d 111]}=\frac{1}{2}\left(f_{2} D_{111}(1,2,3,4)-C_{111}(1,3,4)+C_{111}(1,2,4)\right), \\
& R_{3}^{[d 111]}=\frac{1}{2}\left(f_{3} D_{111}(1,2,3,4)-C_{111}(1,2,4)+C_{111}(1,2,3)\right) . \\
& R_{1}^{[d 222]}=\frac{1}{2}\left(f_{1} D_{222}(1,2,3,4)+C_{111}(1,3,4)-C_{111}(2,3,4)\right), \\
& R_{2}^{[d 222]}=\frac{1}{2}\left(f_{2} D_{222}(1,2,3,4)-C_{111}(1,3,4)+C_{222}(1,2,4)-6 D_{0022}(1,2,3,4)\right) \text {, } \\
& R_{3}^{[d 222]}=\frac{1}{2}\left(f_{3} D_{222}(1,2,3,4)-C_{222}(1,2,4)+C_{222}(1,2,3)\right) . \\
& R_{1}^{[d 333]}=\frac{1}{2}\left(f_{1} D_{333}(1,2,3,4)+C_{222}(1,3,4)-C_{222}(2,3,4)\right), \\
& R_{2}^{[d 333]}=\frac{1}{2}\left(f_{2} D_{333}(1,2,3,4)-C_{222}(1,3,4)+C_{222}(1,2,4)\right), \\
& R_{3}^{[d 333]}=\frac{1}{2}\left(f_{3} D_{333}(1,2,3,4)-C_{222}(1,2,4)-6 D_{0033}(1,2,3,4)\right) . \\
& R_{1}^{[d 112]}=\frac{1}{2}\left(f_{1} D_{112}(1,2,3,4)+C_{111}(1,3,4)-C_{1}(2,3,4)-4 D_{0012}(1,2,3,4)\right), \\
& R_{2}^{[d 112]}=\frac{1}{2}\left(f_{2} D_{112}(1,2,3,4)-C_{111}(1,3,4)+C_{112}(1,2,4)-2 D_{0011}(1,2,3,4)\right) \text {, } \\
& R_{3}^{[d 112]}=\frac{1}{2}\left(f_{3} D_{112}(1,2,3,4)-C_{112}(1,2,4)+C_{112}(1,2,3)\right) . \\
& R_{1}^{[d 113]}=\frac{1}{2}\left(f_{1} D_{113}(1,2,3,4)+C_{112}(1,3,4)-C_{2}(2,3,4)-4 D_{0013}(1,2,3,4)\right), \\
& R_{2}^{[d 113]}=\frac{1}{2}\left(f_{2} D_{113}(1,2,3,4)-C_{112}(1,3,4)+C_{112}(1,2,4)\right), \\
& R_{3}^{[d 113]}=\frac{1}{2}\left(f_{3} D_{113}(1,2,3,4)-C_{112}(1,2,4)-2 D_{0011}(1,2,3,4)\right) . \\
& R_{1}^{[d 122]}=\frac{1}{2}\left(f_{1} D_{122}(1,2,3,4)+C_{111}(1,3,4)+C_{11}(2,3,4)-2 D_{0022}(1,2,3,4)\right), \\
& R_{2}^{[d 122]}=\frac{1}{2}\left(f_{2} D_{122}(1,2,3,4)-C_{111}(1,3,4)+C_{122}(1,2,4)-4 D_{0012}(1,2,3,4)\right) \text {, } \\
& R_{3}^{[d 122]}=\frac{1}{2}\left(f_{3} D_{122}(1,2,3,4)-C_{122}(1,2,4)+C_{122}(1,2,3)\right) .
\end{aligned}
$$




$$
\begin{aligned}
& R_{1}^{[d 133]}=\frac{1}{2}\left(f_{1} D_{133}(1,2,3,4)+C_{122}(1,3,4)+C_{22}(2,3,4)-2 D_{0033}(1,2,3,4)\right), \\
& R_{2}^{[d 133]}=\frac{1}{2}\left(f_{2} D_{133}(1,2,3,4)-C_{122}(1,3,4)+C_{122}(1,2,4)\right), \\
& R_{3}^{[d 133]}=\frac{1}{2}\left(f_{3} D_{133}(1,2,3,4)-C_{122}(1,2,4)-4 D_{0013}(1,2,3,4)\right) . \\
& R_{1}^{[d 223]}=\frac{1}{2}\left(f_{1} D_{223}(1,2,3,4)+C_{112}(1,3,4)-C_{112}(2,3,4)\right), \\
& R_{2}^{[d 223]}=\frac{1}{2}\left(f_{2} D_{223}(1,2,3,4)-C_{112}(1,3,4)+C_{222}(1,2,4)-4 D_{0023}(1,2,3,4)\right) \text {, } \\
& R_{3}^{[d 223]}=\frac{1}{2}\left(f_{3} D_{223}(1,2,3,4)-C_{222}(1,2,4)-2 D_{0022}(1,2,3,4)\right) . \\
& R_{1}^{[d 233]}=\frac{1}{2}\left(f_{1} D_{233}(1,2,3,4)+C_{122}(1,3,4)-C_{122}(2,3,4)\right), \\
& R_{2}^{[d 233]}=\frac{1}{2}\left(f_{2} D_{233}(1,2,3,4)-C_{122}(1,3,4)+C_{222}(1,2,4)-2 D_{0033}(1,2,3,4)\right) \text {, } \\
& R_{3}^{[d 233]}=\frac{1}{2}\left(f_{3} D_{233}(1,2,3,4)-C_{222}(1,2,4)-4 D_{0023}(1,2,3,4)\right) . \\
& R_{1}^{[d 123]}=\frac{1}{2}\left(f_{1} D_{123}(1,2,3,4)+C_{112}(1,3,4)+C_{12}(2,3,4)-2 D_{0023}(1,2,3,4)\right), \\
& R_{2}^{[d 123]}=\frac{1}{2}\left(f_{2} D_{123}(1,2,3,4)-C_{112}(1,3,4)+C_{122}(1,2,4)-2 D_{0013}(1,2,3,4)\right) \text {, } \\
& R_{3}^{[d 123]}=\frac{1}{2}\left(f_{3} D_{123}(1,2,3,4)-C_{122}(1,2,4)-2 D_{0012}(1,2,3,4)\right) . \\
& R_{1}^{[d 001]}=\frac{1}{2}\left(f_{1} D_{001}(1,2,3,4)+C_{001}(1,3,4)+C_{00}(2,3,4)-2 D_{0000}(1,2,3,4)\right), \\
& R_{2}^{[d 001]}=\frac{1}{2}\left(f_{2} D_{001}(1,2,3,4)-C_{001}(1,3,4)+C_{001}(1,2,4)\right), \\
& R_{3}^{[d 001]}=\frac{1}{2}\left(f_{3} D_{001}(1,2,3,4)-C_{001}(1,2,4)+C_{001}(1,2,3)\right) . \\
& R_{1}^{[d 002]}=\frac{1}{2}\left(f_{1} D_{002}(1,2,3,4)+C_{001}(1,3,4)-C_{001}(2,3,4)\right), \\
& R_{2}^{[d 002]}=\frac{1}{2}\left(f_{2} D_{002}(1,2,3,4)-C_{001}(1,3,4)+C_{002}(1,2,4)-2 D_{0000}(1,2,3,4)\right) \text {, } \\
& R_{3}^{[d 002]}=\frac{1}{2}\left(f_{3} D_{002}(1,2,3,4)-C_{002}(1,2,4)+C_{002}(1,2,3)\right) . \\
& R_{1}^{[d 003]}=\frac{1}{2}\left(f_{1} D_{003}(1,2,3,4)+C_{002}(1,3,4)-C_{002}(2,3,4)\right), \\
& R_{2}^{[d 003]}=\frac{1}{2}\left(f_{2} D_{003}(1,2,3,4)-C_{002}(1,3,4)+C_{002}(1,2,4)\right), \\
& R_{3}^{[d 003]}=\frac{1}{2}\left(f_{3} D_{003}(1,2,3,4)-C_{002}(1,2,4)-2 D_{0000}(1,2,3,4)\right) .
\end{aligned}
$$


In addition to the relations detailed in Table A.2 we also have the following $D$-dependent relations, obtained by contracting with the metric tensor. Of these only Eqs. (A.51) and (A.61) are really necessary, since the others are determined by the relations in Table A.2. Nevertheless the redundancy provides a good check of the implementation.

$$
\begin{aligned}
& D_{0000}(1,2,3,4)=\frac{1}{2(D-1)}\left(2 m_{1}^{2} D_{00}(1,2,3,4)+C_{00}(2,3,4)\right. \\
- & \left.f_{1} D_{001}(1,2,3,4)-f_{2} D_{002}(1,2,3,4)-f_{3} D_{003}(1,2,3,4)\right) \\
& D_{0011}(1,2,3,4)=\frac{1}{2(D-1)}\left(2 m_{1}^{2} D_{11}(1,2,3,4)+C_{0}(2,3,4)\right. \\
- & \left.f_{1} D_{111}(1,2,3,4)-f_{2} D_{112}(1,2,3,4)-f_{3} D_{113}(1,2,3,4)\right) \\
& D_{0012}(1,2,3,4)=\frac{1}{2(D-1)}\left(2 m_{1}^{2} D_{12}(1,2,3,4)-C_{1}(2,3,4)\right. \\
- & \left.f_{1} D_{112}(1,2,3,4)-f_{2} D_{122}(1,2,3,4)-f_{3} D_{123}(1,2,3,4)\right) \\
& D_{0013}(1,2,3,4)=\frac{1}{2(D-1)}\left(2 m_{1}^{2} D_{13}(1,2,3,4)-C_{2}(2,3,4)\right. \\
- & \left.f_{1} D_{113}(1,2,3,4)-f_{2} D_{123}(1,2,3,4)-f_{3} D_{133}(1,2,3,4)\right) \\
& D_{0022}(1,2,3,4)=\frac{1}{2(D-1)}\left(2 m_{1}^{2} D_{22}(1,2,3,4)+C_{11}(2,3,4)\right. \\
- & \left.f_{1} D_{122}(1,2,3,4)-f_{2} D_{222}(1,2,3,4)-f_{3} D_{223}(1,2,3,4)\right) \\
& D_{0023}(1,2,3,4)=\frac{1}{2(D-1)}\left(2 m_{1}^{2} D_{23}(1,2,3,4)+C_{12}(2,3,4)\right. \\
- & \left.f_{1} D_{123}(1,2,3,4)-f_{2} D_{223}(1,2,3,4)-f_{3} D_{233}(1,2,3,4)\right) \\
& D_{0033}(1,2,3,4)=\frac{1}{2(D-1)}\left(2 m_{1}^{2} D_{33}(1,2,3,4)+C_{22}(2,3,4)\right. \\
- & \left.f_{1} D_{133}(1,2,3,4)-f_{2} D_{233}(1,2,3,4)-f_{3} D_{333}(1,2,3,4)\right) \\
& D_{001}(1,2,3,4)=\frac{1}{2(D-2)}\left(2 m_{1}^{2} D_{1}(1,2,3,4)-C_{0}(2,3,4)\right. \\
- & \left.f_{1} D_{11}(1,2,3,4)-f_{2} D_{12}(1,2,3,4)-f_{3} D_{13}(1,2,3,4)\right) \\
& D_{002}(1,2,3,4)=\frac{1}{2(D-2)}\left(2 m_{1}^{2} D_{2}(1,2,3,4)+C_{1}(2,3,4)\right. \\
- & \left.f_{1} D_{12}(1,2,3,4)-f_{2} D_{22}(1,2,3,4)-f_{3} D_{23}(1,2,3,4)\right) \\
& D_{003}(1,2,3,4)=\frac{1}{2(D-2)}\left(2 m_{1}^{2} D_{3}(1,2,3,4)+C_{2}(2,3,4)\right. \\
- & \left.f_{1} D_{13}(1,2,3,4)-f_{2} D_{23}(1,2,3,4)-f_{3} D_{33}(1,2,3,4)\right) \\
& D_{00}(1,2,3,4)=\frac{1}{2(D-3)}\left(2 m_{1}^{2} D_{0}(1,2,3,4)+C_{0}(2,3,4)\right. \\
- & \left.f_{1} D_{1}(1,2,3,4)-f_{2} D_{2}(1,2,3,4)-f_{3} D_{3}(1,2,3,4)\right) \\
&
\end{aligned}
$$




\section{Appendix B. Rational terms of specific tensor integrals}

As an illustration, we present results of the computation of rational parts of some of the tensor integrals. We begin with the rank-two two-point function. To calculate the rational part, we perform the reduction of the integral, along the lines described above, and trace the part of the reduction that depends on the $(D-4)$-component of the loop momentum. Considering the rank-two two-point integral

$$
I_{2}^{\mu \nu}=\int \frac{\mathrm{d}^{D} l}{(2 \pi)^{D}} \frac{l^{\mu} l^{\nu}}{d_{0} d_{1}}
$$

where $d_{i}=\left(l+q_{i}\right)^{2}-m_{i}^{2}$ we find its rational part

$$
\mathcal{R}\left[I_{2}\right]_{\mu \nu}=i c_{\Gamma}\left(\frac{\omega_{\mu \nu}}{3}+\frac{g_{\mu \nu}^{-2 \epsilon}}{2 \epsilon}\right) F_{01} .
$$

In Eq. (B.2), $\omega^{\mu \nu}$ is the metric tensor of the transverse space $\omega_{\mu \nu}\left(q_{1}-q_{0}\right)^{\mu}=$ 0 and $g_{\mu \nu}^{-2 \epsilon}$ is the metric tensor of the $(D-4)$-dimensional space. Also, we introduced the short-hand notation

$$
F_{i j}=\frac{m_{i}^{2}+m_{j}^{2}}{2}-\frac{\left(q_{i}-q_{j}\right)^{2}}{6}
$$

and the loop factor $c_{\Gamma}$ reads,

$$
c_{\Gamma}=\frac{1}{(4 \pi)^{2-\epsilon}} \frac{\Gamma(1+\epsilon) \Gamma^{2}(1-\epsilon)}{\Gamma(1-2 \epsilon)}=\frac{(4 \pi)^{\epsilon}}{16 \pi^{2}} \frac{1}{\Gamma(1-\epsilon)}+\mathcal{O}\left(\epsilon^{3}\right) .
$$

We note that the presence of the $\epsilon$-dependent part of the metric tensor in the result is the reflection of the fact that the rank-two two-point function is divergent. This term does not contribute when $I_{\mu \nu}$ is contracted with the four-dimensional vectors, as it is often the case, but when $I_{\mu \nu}$ is contracted with the full $D$ dimensional metric tensor, it does contribute. In fact, the two terms shown in the right hand side of Eq. (B.2) can be identified with the two contributions to the rational part $\mathcal{R}_{1,2}$, discussed at the end of Section 5.3.

For the three-point functions, we need to consider the rank-two and the rank-three tensor integrals. For the rank-two three-point function

$$
I_{3}^{\mu \nu}=\int \frac{\mathrm{d}^{D} l}{(2 \pi)^{D}} \frac{l^{\mu} l^{\nu}}{d_{0} d_{1} d_{2}}
$$

the rational part is remarkably simple. It reads

$$
\mathcal{R}\left[I_{3}\right]_{\mu \nu}=i c_{\Gamma}\left(\frac{\omega_{\mu \nu}\left(q_{1}, q_{2}\right)}{4}+\frac{g_{\mu \nu}^{-2 \epsilon}}{4 \epsilon}\right) .
$$

However, the rational part becomes significantly more complex for the rankthree three-point function

$$
I_{3}^{\mu \nu \alpha}=\int \frac{\mathrm{d}^{D} l}{(2 \pi)^{D}} \frac{l^{\mu} l^{\nu} l^{\alpha}}{d_{0} d_{1} d_{2}} .
$$


We find

$$
\begin{aligned}
\mathcal{R}\left[I_{3}\right]^{\mu \nu \alpha} & =i c_{\Gamma}\left(\sum_{i=1}^{2} v_{i}^{\mu} v_{i}^{\nu} v_{i}^{\alpha} c_{i i i}+\sigma_{2}^{\mu} \sigma_{2}^{\nu} \sigma_{2}^{\alpha} c_{000}\right. \\
& \left.+\sum_{i=1}^{2} v_{i}^{\{\alpha} c_{\perp, i}^{\mu \nu\}}+\sigma_{2}^{\{\alpha} c_{\perp, 0}^{\mu \nu\}}+t_{3,1}^{\{\mu \nu} v^{\alpha\}}\right),
\end{aligned}
$$

and the curly braces $\{\ldots\}$ indicate symmetrization over the three cyclic permutations of the indices. As usual $v_{i}^{\mu}$ are the basis vectors of the physical space in van Neerven - Vermaseren basis, $\sigma_{n}=\sum_{i=1}^{n} v_{i}$ and

$$
\begin{array}{ll}
c_{111}=\frac{\Delta_{2} F_{02}}{6 q_{2}^{2}}, & c_{222}=\frac{\Delta_{2} F_{01}}{6 q_{1}^{2}}, \quad c_{000}=-\frac{\Delta_{2} F_{12}}{6\left(q_{2}-q_{1}\right)^{2}}, \\
c_{\perp, 1}^{\alpha \beta}=t_{3,2}^{\alpha \beta} \frac{F_{02}}{2}, & c_{\perp, 2}^{\alpha \beta}=t_{3,2}^{\alpha \beta} \frac{F_{01}}{2}, \quad c_{\perp, 0}^{\alpha \beta}=-t_{3,2}^{\alpha \beta} \frac{F_{12}}{2} .
\end{array}
$$

The Kronecker delta contracted with the two momenta is denoted by $\Delta_{2}=$ $\Delta\left(q_{1}, q_{2}\right)=\delta_{q_{1} q_{2}}^{q_{1} q_{2}}$, see Section 3 for details. Also, we introduced

$$
t_{3,1}^{\alpha \beta}=\frac{\omega^{\alpha \beta}\left(q_{1}, q_{2}\right)}{4}+\frac{g_{-2 \epsilon}^{\mu \nu}}{4 \epsilon}, \quad t_{3,2}^{\alpha \beta}=\frac{\omega^{\alpha \beta}\left(q_{1}, q_{2}\right)}{3}+\frac{g_{-2 \epsilon}^{\mu \nu}}{2 \epsilon},
$$

and the auxiliary vector

$$
v^{\mu}=\frac{1}{2} \sum_{i=1}^{2} v_{i}^{\mu}\left(m_{i}^{2}-m_{0}^{2}-q_{i}^{2}\right) .
$$

A similar situation occurs with the four-point functions. For the rational part of the rank-three four-point function

$$
I_{4}^{\mu \nu \alpha}=\int \frac{\mathrm{d}^{D} l}{(2 \pi)^{D}} \frac{l^{\mu} l^{\nu} l^{\alpha}}{d_{0} d_{1} d_{2} d_{3}},
$$

we obtain

$$
\mathcal{R}\left[I_{4}\right]^{\mu \nu \alpha}=i c_{\Gamma}\left(\sum_{i=1}^{3} v_{i}^{\mu} v_{i}^{\nu} v_{i}^{\alpha} d_{i i i}+\sigma_{3}^{\mu} \sigma_{3}^{\nu} \sigma_{3}^{\alpha} d_{000}\right),
$$

where

$$
d_{i i i}=\frac{\Delta_{3}}{8 Q_{i i}}, \quad d_{000}=-\frac{\Delta_{3}}{8 Q} .
$$

Here, we use the following general notation. Consider an $n+1$-point function with momenta $q_{i}$. The Gram-determinant matrix is given by $G_{i j}=\left(q_{i} \cdot q_{j}\right)$. We denote the elements of the inverse Gram matrix by

$$
G_{i j}^{-1}=\frac{Q_{i j}}{\Delta_{n}},
$$


where $\Delta_{n}=\delta_{q_{1} \ldots q_{n}}^{q_{1} \ldots q_{n}}$. With this notation, $Q_{i j}$ equals to $(-1)^{i+j}$ times the determinant of a minor of the Gram matrix obtained by removing the $i$-th row and the $j$-th column. These minors can be obtained from the scalar product of the van Neerven-Vermaseren basis vectors

$$
Q_{i j}=\Delta_{n} v_{i} v_{j}
$$

We also employ the short-hand notation

$$
Q_{i}=\sum_{j=1}^{n} Q_{i j}, \quad Q=\sum_{i=1}^{n} Q_{i}
$$

We note that, while the rational part of the rank-three four-point function and Eq. (B.13) is remarkably compact, a similar formula for the rank-four fourpoint is much more involved and we do not present it here.

\section{Appendix C. Cutkosky rules}

Scattering amplitudes in quantum field theories are functions of scalar products of four-momenta of scattered particles. It is possible to complexify these scalar products and study how scattering amplitudes depend on them. In particular, a $2 \rightarrow 2$ scattering process of particles of mass $m$ can be fully described by two independent Mandelstam variables $s$ and $t=-\left(s-4 m^{2}\right) / 2\left(1-\cos \theta_{\mathrm{cms}}\right)$, where $\theta_{\mathrm{cms}}$ is the scattering angle in the center-of-mass frame. It is useful to consider the scattering amplitude as a function of $s$, keeping $\theta_{\mathrm{cms}}$ fixed. It follows from the unitarity of the scattering matrix $S$ that the scattering amplitude is an analytic function in the complex $s$-plane with possible single-particle poles in non-physical regions and branch cuts corresponding to multi-particle thresholds. Indeed, since $S^{\dagger} S=1, S=1+i T$,

$$
2 \operatorname{Im} T=-i\left(T-T^{\dagger}\right)=T^{\dagger} T=T T^{\dagger} .
$$

Taking the matrix element of both sides of the above equation between twoparticle states and using the completeness relation, we find

$$
2 \operatorname{Im}\left\langle p_{3}, p_{4}|T| p_{1}, p_{2}\right\rangle=\sum_{n}\left\langle n|T| p_{3}, p_{4}\right\rangle^{*}\left\langle n|T| p_{1}, p_{2}\right\rangle
$$

The scattering amplitude $M(s, t)$ is defined by factoring the energy-momentum conserving $\delta$-function from the matrix element of the $T$-matrix, $\left\langle p_{3}, p_{4}|T| p_{1}, p_{2}\right\rangle=$ $(2 \pi)^{4} \delta\left(p_{1}+p_{2}-p_{3}-p_{4}\right) M(s, t)$. As the scattering energy increases, the imaginary part of the scattering amplitude receives contributions from intermediate states of higher multiplicities. As a result $M(s, t)$ has branch cuts in the complex $s$-plane at $(n m)^{2}$, with $n=(2,3,4, \ldots)$. The discontinuity of the amplitude $M(s, t)$ at these cuts is given by Eq. (C.2). The analysis of the analytic properties of multi-particle amplitudes based on unitarity and crossing 
symmetry becomes more and more cumbersome with increasing number of external particles. In perturbation theory, however, the Cutkosky formula [150] $1 /\left(p^{2}-m^{2}+i \delta\right) \rightarrow(-2 \pi i) \delta\left(p^{2}-m^{2}\right) \theta\left(p_{0}\right)$ provides us with a simple recipe for calculating discontinuities across branch cuts of multi-loop, multi-leg Feynman diagrams. In this Appendix, we discuss the origin of the Cutkosky rule using the example of a two-point function. Our presentation follows Ref. [151] where further details can be found.

Consider a diagram that contributes to a correlation function of two scalar fields in $\varphi^{3}$-theory

$$
\begin{aligned}
& \mathcal{I}^{(2)}\left(p^{2}, m^{2}\right)=\int \frac{\mathrm{d}^{4} l}{(2 \pi)^{4}} \frac{1}{D_{1} D_{2}}, \\
& D_{1}=l^{2}-m^{2}+i 0, \quad D_{2}=(p-l)^{2}-m^{2}+i 0 .
\end{aligned}
$$

This diagram is an analytic function of $p^{2}$, with a cut along the positive real axis, starting at $p^{2}=4 \mathrm{~m}^{2}$. To understand the origin of the discontinuity across the cut, we start computing the integral in Eq. (C.3) by integrating over $l_{0}$. We do so by using the residue theorem. We work in a reference frame where $p=\left(p_{0}, \overrightarrow{0}\right)$ and $p_{0}>0$. There are four poles that we need to consider:

$$
\begin{array}{lll}
D_{1}: & \left.a_{1}\right) l_{0}=\epsilon_{l}-i 0, & \left.b_{1}\right) l_{0}=-\epsilon_{l}+i 0, \\
D_{2}: & \left.a_{2}\right) l_{0}=p_{0}+\epsilon_{l}-i 0, & \left.b_{2}\right) l_{0}=p_{0}-\epsilon_{l}+i 0,
\end{array}
$$

where $\epsilon_{l}=\sqrt{\vec{l}^{2}+m^{2}}$. From Eq. (C.4), it follows that two $l_{0}$-poles are located above and two $l_{0}$-poles are located below the real axis, see the upper inset in Fig. C.1. We can also compute the distance between the poles in Eq. (C.4). We find that the distance between the poles $b_{2}$ and $a_{1}$ can vanish, while distances between all other poles are larger than zero. This observation has important consequences. Suppose we start with a $p_{0}$ value such that $0<p_{0}<2 m$. In that case poles $b_{1,2}$ are above the negative real axis and poles $a_{1,2}$ are below the positive real axis. It is clear that the integration contour can be deformed to complex $l_{0}$-values such that $\operatorname{Re}\left(l_{0}\right)<0, \operatorname{Im}\left(l_{0}\right)<0$ and $\operatorname{Re}\left(l_{0}\right)>0, \operatorname{Im}\left(l_{0}\right)>0$. Once the integration contour is deformed, it becomes clear that the integral can not develop a discontinuity in $p^{2}$. Indeed, the discontinuity is the result of differing values of the integral when computed with $p_{0} \rightarrow p_{0} \pm i \delta, \delta \rightarrow 0$. The location of the poles changes when $p_{0}$ is substituted by $p_{0} \pm i \delta$. However, because the integration contour is far away from the poles, the result of the integration is not affected.

This argument fails if the integration contour cannot be moved away from the poles. In our example, this happens if the integration contour is pinched between the two poles, so that they are on top of each other. This can only happen for the two poles $b_{2}$ and $a_{1}$ in Eq. (C.4). The distance between the poles $b_{2}$ and $a_{1}$ vanishes if $p_{0}=2 \epsilon_{l}$; this can only happen if $p_{0} \geq 2 \mathrm{~m}$. The Lorentz-invariant form of this condition is $p^{2} \geq 4 m^{2}$, which is the point in the complex $p^{2}$-plane where the two-particle cut starts. 


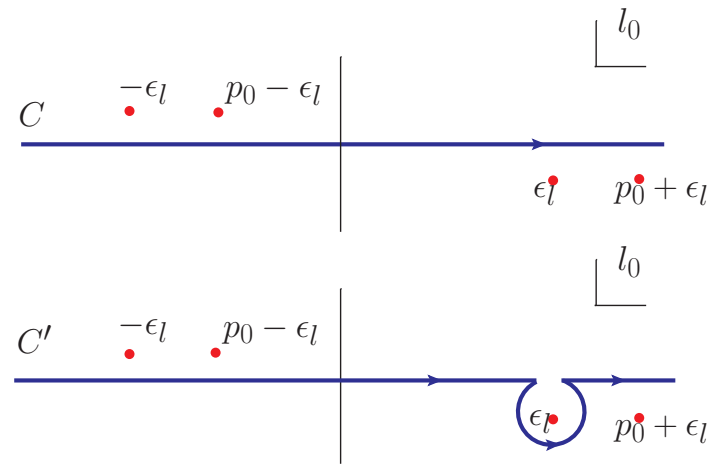

Figure C.1: Integration contours in the $l_{0}$ plane. See text for details.

To compute the discontinuity, we go back to Eq. (C.3) and write it as an integral over the contour $C$ shown in the upper inset in Fig. C.1

$$
\mathcal{I}^{(2)}\left(p^{2}, m^{2}\right)=\int \frac{\mathrm{d}^{3} \vec{l}}{(2 \pi)^{3}} \int_{C} \frac{\mathrm{d} l_{0}}{(2 \pi)} \frac{1}{\left(l_{0}^{2}-\epsilon_{l}^{2}\right)\left(\left(l_{0}-p_{0}\right)^{2}-\epsilon_{l}^{2}\right)} .
$$

We can write this integral as the sum of two terms: the integral over a contour $C^{\prime}$ (see the lower inset in Fig. C.1 ) where pinching does not occur and therefore no discontinuity is present, and the residue at the pole $a_{1}$

$$
\mathcal{I}^{(2)}=\int \frac{\mathrm{d}^{3} \vec{l}}{(2 \pi)^{3}}\left[\int_{C^{\prime}} \frac{\mathrm{d} l_{0}}{(2 \pi)} \ldots+\oint_{\gamma_{a_{1}}} \frac{\mathrm{d} l_{0}}{(2 \pi)} \ldots\right] .
$$

The integral over $\gamma_{a_{1}}$ can be written as

$$
\oint_{\gamma_{a_{1}}} \frac{\mathrm{d} l_{0}}{(2 \pi)} \frac{1}{\left(l_{0}^{2}-\epsilon_{l}^{2}\right)\left(\left(l_{0}-p_{0}\right)^{2}-\epsilon_{l}^{2}\right)}=\int \frac{\mathrm{d} l_{0}}{(2 \pi)} \frac{(-2 \pi i) \delta_{+}\left(l^{2}-m^{2}\right)}{\left(\left(l_{0}-p_{0}\right)^{2}-\epsilon_{l}^{2}\right)},
$$

where $\delta_{+}\left(l^{2}-m^{2}\right)=\delta\left(l^{2}-m^{2}\right) \theta\left(l_{0}\right)$. The discontinuity of the integral in Eq. (C.7) can be computed directly. For our choice of the reference frame, $p^{2} \pm i \delta \equiv p_{0}^{2} \pm i \delta$. Therefore,

$$
\begin{aligned}
\operatorname{Disc}\left[\mathcal{I}_{2}\right] & =\mathcal{I}_{2}\left(p^{2}+i \delta\right)-\mathcal{I}_{2}\left(p^{2}-i \delta\right) \\
& =\int \frac{\mathrm{d}^{4} l}{(2 \pi)^{4}}(-2 \pi i) \delta_{+}\left(l^{2}-m^{2}\right) \times\left[\frac{1}{\left(l_{0}-p_{0}-i \delta\right)^{2}-\epsilon_{l}^{2}}-\text { c.c }\right] \\
& =\int \frac{\mathrm{d}^{4} l}{(2 \pi)^{4}}(-2 \pi i) \delta_{+}\left(l^{2}-m^{2}\right)\left[\frac{1}{(l-p)^{2}-m^{2}+i \delta}-\text { c.c. }\right] .
\end{aligned}
$$


As the final step, we use

$$
\frac{1}{x+i \delta}=\mathrm{P}\left[\frac{1}{x}\right]-i \pi \delta(x) .
$$

to simplify the difference of the two terms in square brackets and find

$$
\operatorname{Disc}\left[\mathcal{I}_{2}\right]=\int \frac{\mathrm{d}^{4} l}{(2 \pi)^{4}}(-2 \pi i) \delta_{+}\left(l^{2}-m^{2}\right)(-2 \pi i) \delta_{+}\left((l-p)^{2}-m^{2}\right) .
$$

Hence, the discontinuity of the integral $\mathcal{I}_{2}$ is obtained by replacing both propagators with $\delta$-functions, in agreement with the Cutkosky rule.

We now discuss how this result generalizes. We begin with the formulation of a more thorough condition for the appearance of the discontinuities in one-loop Feynman diagrams. As follows from the above discussion, discontinuities appear when the integration contour becomes trapped between poles of the integrand of a given Feynman diagram. To streamline a discussion of when this happens, we go back to our example and combine denominators using Feynman parameters

$$
\mathcal{I}^{(2)}\left(p^{2}, m^{2}\right)=\int \frac{\mathrm{d}^{4} l}{(2 \pi)^{4}} \frac{1}{D_{1} D_{2}}=\int \frac{\mathrm{d}^{4} l}{(2 \pi)^{4}} \int \mathrm{d} \alpha_{1} \mathrm{~d} \alpha_{2} \frac{\delta\left(1-\sum \alpha_{i}\right)}{D^{2}},
$$

where $D=\alpha_{1} D_{1}+\alpha_{2} D_{2}$. Singularities in the above expression appear, if $D=0$. The necessary condition for that is $D_{1}\left(l^{*}\right)=0, D_{2}\left(l^{*}\right)=0$. Since $D$ is a quadratic polynomial in the loop momentum $l$, equation $D(l)=0$ contains two solutions which, in general, are different. In such a situation, the integration contour can be deformed, the pinch singularity can be avoided and no discontinuity appears. This does not occur if the two solutions of the quadratic equation coincide. Suppose we write

$$
D(l)=\left(l_{0}-a(\vec{l}, p, m)\right)\left(l_{0}-b(\vec{l}, p, m)\right),
$$

where $a$ and $b$ are functions of the three-momentum $\vec{l}$, external momentum $p$ and the mass $m$. The condition that the poles coincide is

$$
\left.\frac{\partial D}{\partial l_{0}}\right|_{l_{0}=a \text { or } b}=0 .
$$

The covariant generalization of this equation is straightforward

$$
\left.\frac{\partial D}{\partial l^{\mu}}\right|_{l=l^{*}}=0
$$

Because $D(l)=\alpha_{1} D_{1}+\alpha_{2} D_{2}$ and $D_{1,2}=\left(l+q_{1,2}\right)^{2}-m^{2}$, we find

$$
\left.\frac{\partial D}{\partial l_{\mu}}\right|_{l=l^{*}}=0 \Rightarrow \alpha_{1} l_{1}^{*}+\alpha_{2} l_{2}^{*}=0,
$$


where $l_{1,2}^{*}=l^{*}+q_{1,2}$ and $l^{*}$ is such that $D_{1}\left(l^{*}\right)=D_{2}\left(l^{*}\right)=0$. If Eq. (C.15) has solutions for real values of the loop momentum and for physical values of Feynman parameters $0 \leq \alpha_{1,2} \leq 1$, there is a pinch singularity and, correspondingly, a discontinuity in a given Feynman diagram.

The generalization of the above discussion leads to Landau equations [46] (see Ref. [152] for a pedagogical introduction). To present these equations, we consider an $L$-loop Feynman integral with $N$ propagators $D_{i}=q_{i}^{2}-m_{i}^{2}, i \in N$

$$
\mathcal{I}\left(\left\{p_{i}\right\},\left\{m_{i}\right\}\right)=\int \prod_{k=1}^{L} \frac{d^{4} l_{k}}{(2 \pi)^{4}} \frac{\mathcal{N}\left(\left\{l_{i}\right\}\left\{p_{i}\right\}\right)}{\prod_{i=1}^{N} D_{i}} .
$$

The set of equations that determines the positions of the pinch singularities of the integral (C.16) that lead to a discontinuity across an $r$-particle cut is

$$
\begin{gathered}
D_{i}=q_{i}^{2}-m_{i}^{2}=0, \quad(i=1,2, \ldots, r \leq 4 L), \\
\sum_{i \subset \text { loop j }} \alpha_{i}^{(j)} q_{i}=0 \quad \text { for every loop. }
\end{gathered}
$$

The first set of Landau equations Eq. (C.17) selects a finite number of vanishing propagators, i.e. defines a cut. This is a necessary condition for a Feynman integral to develop a singularity and to have a discontinuity in a particular channel. As we saw in the explicit example, the second set of equations (C.18) is a condition that the singularities trap the integration contour.

The Landau equations provide us with sets of homogeneous linear equations for the coefficients $\alpha_{i}^{(j)}$. The condition to have non-trivial solutions requires that the corresponding determinants vanish. The equations given by the vanishing determinants allow us to calculate the positions of the branch point singularities associated with a particular channel.

To illustrate how the Landau equations work, we go back to our example the integral $\mathcal{I}_{2}$. We slightly generalize it by considering two different masses in the inverse Feynman propagators $D_{1}$ and $D_{2}$. The position of the singularity for the double cut is given by the Landau equations

$$
l^{2}-m_{1}^{2}=0, \quad(l-p)^{2}-m_{2}^{2}=0, \quad\left(\alpha_{1}+\alpha_{2}\right) l^{\mu}-\alpha_{2} p^{\mu}=0 .
$$

Contracting the last equation with $l$ and $p$, we obtain a linear system of equations for $\alpha_{1,2}$

$$
\begin{aligned}
\left(\alpha_{1}+\alpha_{2}\right) m_{1}^{2}-\alpha_{2}(l \cdot p) & =0 \\
\left(\alpha_{1}+\alpha_{2}\right)(l \cdot p)-\alpha_{2} p^{2} & =0 .
\end{aligned}
$$

These equations have non-trivial solutions for $\alpha_{1}, \alpha_{2}$ provided that the corresponding determinant vanishes. Hence,

$$
m_{1}^{2} p^{2}-(l \cdot p)^{2}=0, \quad \text { and } \quad l \cdot p=\frac{1}{2}\left(m_{1}^{2}+p^{2}-m_{2}^{2}\right) .
$$


Solving this equation for $p^{2}$ we obtain

$$
p_{ \pm}^{2}=\left(m_{1} \pm m_{2}\right)^{2} .
$$

In spite of the two solutions, the physical two-particle threshold is at $p^{2}=p_{+}^{2}$ because we need to satisfy additional constraints $l^{0}>0$ and $p^{0}-l^{0}>0$. This leaves only one solution for the branch cut point $p^{2}=p_{+}^{2}=\left(m_{1}+m_{2}\right)^{2}$.

Having discussed the examples of how Cutkosky rules are used, the reasons discontinuities appear and equations that can be used to find locations of branch cuts, we are now in a position to write down a general formula for the discontinuity of a Feynman diagram in a particular $r$-particle channel. We note that a complete proof of Cutkosky rules is non-trivial; details can be found in $[150,153]$.

To be specific, we imagine that in a general Feynman diagram Eq. (C.16), a discontinuity appears if the first $r$ propagators go on the mass-shell. The discontinuity across this cut can be computed using Cutkosky rules [150]

$$
\operatorname{Disc}[\mathcal{I}]=\int \prod_{k=1}^{L} \frac{d^{4} l_{k}}{(2 \pi)^{4}} \frac{\mathcal{N}\left(\left\{l_{i}\right\}\left\{p_{i}\right\}\right) \prod_{i=1}^{r}(-2 \pi i) \delta^{(+)}\left(q_{i}^{2}-m_{i}^{2}\right)}{\prod_{j=r+1}^{N}\left(q_{j}^{2}-m_{j}^{2}\right)} .
$$

The above equation is the main result of this Appendix. We hope to have sufficiently motivated it with the above discussion. We finish this Appendix with a few remarks.

a) As shown in Eq. (C.1), unitarity implies non-linear relations between scattering amplitudes. Those relations can be used to compute the discontinuities of scattering amplitudes at a given order in the perturbative expansion, in terms of amplitudes at lower orders.

b) Unitarity is built into perturbation theory in an even more detailed manner. It is not necessary for the external legs to have on-shell values. If the Landau equations have solutions, the Cutkosky formula provides the correct singularities also in the case of off-shell legs. Only the cut lines need be on-shell.

c) The diagrammatic version of the Cutkosky formula Eq. (C.23) can be applied to scattering amplitudes, leading to generalized unitarity relations. Consider the triangle singularity. It appears in many diagrams. Specializing to one loop and summing up all diagrams with such a discontinuity, we obtain

$$
\begin{gathered}
\operatorname{Disc}_{l \rightarrow l_{n_{1} n_{2} n_{3}}}\left[A_{n}^{1-\text { loop }}\right]=\sum_{\text {states }} \int \frac{\mathrm{d}^{4} l}{(2 \pi)^{4}} \prod_{i=1}^{3}(-2 \pi i) \delta_{+}\left(l_{i}^{2}-m_{i}^{2}\right) \\
\times A_{n_{1}}^{\text {tree }}\left(-l_{3}, l_{1}\right) A_{n_{2}}^{\text {tree }}\left(-l_{1}, l_{2}\right) A_{n_{3}}^{\text {tree }}\left(-l_{2}, l_{3}\right)
\end{gathered}
$$

where $A_{n}^{1-\text { loop }}$ and $A^{\text {tree }}$ are one-loop and tree scattering on-shell scattering amplitudes. 
d) In $D$-dimensions the maximal possible number of cut propagators of an $L$-loop Feynman diagram is $D \times L$ since in that case all components of the loop momenta are fixed by on-shell constraints. Obviously, when the maximal cut is evaluated, no integration over the loop momentum is needed since the integral reduces to a sum over discrete solutions. For one-loop computations in $D$-dimensions, with $D>4$, at most five cuts are required, since the extra components of the loop momenta decouple from the four-dimensional kinematics.

\section{Appendix D. Spinor Helicity methods}

The main thrust of this review has been the description of methods that are applicable to one-loop amplitudes containing both massless and massive particles. However QCD contains massless gluons and, at energies much larger than their masses, quarks can also be considered massless. Considerable simplification can be achieved in massless amplitudes by the use of spinor helicity methods $[140,154]$. These methods, as they apply to tree diagrams, have been extensively reviewed in refs. [82, 83, 112]. In the main text, Section 9.6, spinorhelicity methods are used in the calculation of a simple one-loop diagram. The purpose of this appendix is to give a short review of these methods to elucidate the examples given in the main text. Reviews of the application of spinor-helicity methods to loop diagrams can also be found in refs. [155, 25, 26].

\section{Appendix D.1. Spinor solutions for massless fermions}

We choose to work in the Weyl representation for the gamma matrices, Eq. (7.12). In this representation upper and lower components have different helicities

$$
\gamma_{R}=\frac{1}{2}\left(1+\gamma_{5}\right)=\left(\begin{array}{ll}
\mathbf{1} & \mathbf{0} \\
\mathbf{0} & \mathbf{0}
\end{array}\right), \quad \gamma_{L}=\frac{1}{2}\left(1-\gamma_{5}\right)==\left(\begin{array}{ll}
\mathbf{0} & \mathbf{0} \\
\mathbf{0} & \mathbf{1}
\end{array}\right) .
$$

The massless spinor solutions of the Dirac equations are (c.f. Eq. (7.13))

$$
u_{+}(p)=\left[\begin{array}{c}
\sqrt{p^{+}} \\
\sqrt{p^{-}} e^{i \varphi_{p}} \\
0 \\
0
\end{array}\right], \quad u_{-}(p)=\left[\begin{array}{c}
0 \\
0 \\
\sqrt{p^{-}} e^{-i \varphi_{p}} \\
-\sqrt{p^{+}}
\end{array}\right],
$$

where

$$
e^{ \pm i \varphi_{p}} \equiv \frac{p^{x} \pm i p^{y}}{\sqrt{\left(p^{x}\right)^{2}+\left(p^{y}\right)^{2}}}=\frac{p^{x} \pm i p^{y}}{\sqrt{p^{+} p^{-}}}, \quad p^{ \pm}=E \pm p^{z} .
$$

In this representation the Dirac conjugate spinors are

$$
\begin{aligned}
& \overline{u_{+}}(p) \equiv u_{+}^{\dagger}(p) \gamma^{0}=\left[0,0, \sqrt{p^{+}}, \sqrt{p^{-}} e^{-i \varphi_{p}}\right] \\
& \overline{u_{-}}(p)=\left[\sqrt{p^{-}} e^{i \varphi_{p}},-\sqrt{p^{+}}, 0,0\right]
\end{aligned}
$$


and the spinors are normalized such that $u_{ \pm}^{\dagger} u_{ \pm}=2 E$. In the massless limit the antiparticle spinors obey the same Dirac equation and we may choose the phase such that particle spinors $u(p)$ and antiparticle spinors $v(p)$ satisfy

$$
u_{ \pm}(p)=v_{\mp}(p) .
$$

We now introduce a bra and ket notation for spinors corresponding to (massless) momenta $p_{i}, i=1,2, \ldots, n$ labeled by the index $i$

$$
\begin{aligned}
& |i+\rangle \equiv|i\rangle \equiv\left|p_{i}+\right\rangle \equiv u_{+}\left(p_{i}\right)=v_{-}\left(p_{i}\right), \\
& |i-\rangle \equiv \mid i] \equiv\left|p_{i}-\right\rangle \equiv u_{-}\left(p_{i}\right)=v_{+}\left(p_{i}\right), \\
& \langle i+| \equiv\left[i \mid \equiv\left\langle p_{i}+\right| \equiv \overline{u_{+}}\left(p_{i}\right)=\overline{v_{-}}\left(p_{i}\right),\right. \\
& \langle i-| \equiv\langle i| \equiv\left\langle p_{i}-\right| \equiv \overline{u_{-}}\left(p_{i}\right)=\overline{v_{+}}\left(p_{i}\right) .
\end{aligned}
$$

We further define the basic spinor products by

$$
\begin{aligned}
\langle i j\rangle & \equiv\langle i-\mid j+\rangle=\overline{u_{-}}\left(p_{i}\right) u_{+}\left(p_{j}\right), \\
{[i j] } & \equiv\langle i+\mid j-\rangle=\overline{u_{+}}\left(p_{i}\right) u_{-}\left(p_{j}\right) .
\end{aligned}
$$

The helicity projection implies that products like $\langle i+\mid j+\rangle$ vanish

$$
\langle i+\mid j+\rangle=[i j\rangle=\langle i-\mid j-\rangle=\langle i j]=0 .
$$

It is also straightforward to verify that the spinor products satisfy the following relations

$$
\langle i j\rangle=-\langle j i\rangle \quad[i j]=-[j i],
$$

which imply

$$
\langle i i\rangle=[i i]=0 .
$$

We will use the first two notations in Eq. (D.6) interchangeably. Thus we may write

$$
\begin{aligned}
\left\langle i-\left|\gamma^{\mu}\right| j-\right\rangle & \equiv\left\langle i\left|\gamma^{\mu}\right| j\right], \\
\left\langle i+\left|\gamma^{\mu}\right| j+\right\rangle & \equiv\left[i\left|\gamma^{\mu}\right| j\right\rangle .
\end{aligned}
$$

\section{Appendix D.2. Spinor products}

For the case where both energies are positive, $p_{i}^{0}>0, p_{j}^{0}>0$, we can write the spinor products explicitly as

$$
\begin{aligned}
& \langle i j\rangle=\sqrt{p_{i}^{-} p_{j}^{+}} e^{i \varphi_{p_{i}}}-\sqrt{p_{i}^{+} p_{j}^{-}} e^{i \varphi_{p_{j}}}=\sqrt{\left|s_{i j}\right|} e^{i \phi_{i j}}, \\
& {[i j]=\sqrt{p_{i}^{+} p_{j}^{-}} e^{-i \varphi_{p_{j}}}-\sqrt{p_{i}^{-} p_{j}^{+}} e^{-i \varphi_{p_{i}}}=-\sqrt{\left|s_{i j}\right|} e^{-i \phi_{i j}},}
\end{aligned}
$$

where $s_{i j}=\left(p_{i}+p_{j}\right)^{2}=2 p_{i} \cdot p_{j}$, and

$$
\cos \phi_{i j}=\frac{p_{i}^{x} p_{j}^{+}-p_{j}^{x} p_{i}^{+}}{\sqrt{\left|s_{i j}\right| p_{i}^{+} p_{j}^{+}}}, \quad \sin \phi_{i j}=\frac{p_{i}^{y} p_{j}^{+}-p_{j}^{y} p_{i}^{+}}{\sqrt{\left|s_{i j}\right| p_{i}^{+} p_{j}^{+}}} .
$$


Thus, the spinor products are, up to a phase, square roots of Lorentz products. For real momenta with positive energy components we have that $\langle i j\rangle^{*}=[j i]$. Note, however, that for complex momenta this is no longer true.

By explicit construction one can show that

$$
|b+\rangle\langle c-|-| c+\rangle\langle b-|=\langle c-\mid b+\rangle \gamma_{R} .
$$

Contracting this equation with $\langle a-|$ from the left and with $|d+\rangle$ from the right, we obtain the Schouten identity

$$
\langle a-\mid b+\rangle\langle c-\mid d+\rangle-\langle a-\mid c+\rangle\langle b-\mid d+\rangle=\langle c-\mid b+\rangle\langle a-\mid d+\rangle .
$$

We can write it more compactly as

$$
\begin{aligned}
& \langle a b\rangle\langle c d\rangle-\langle a c\rangle\langle b d\rangle-\langle c b\rangle\langle a d\rangle=0, \\
& {[a b][c d]-[a c][b d]-[c b][a d]=0 .}
\end{aligned}
$$

Note that the expressions on the left of Eq. (D.17) are totally antisymmetric under the exchange of $a, b, c$. The Schouten identity follows because the totally antisymmetric product of three two-component objects is equal to zero.

The importance of spinor products for describing on-shell scattering amplitudes in gauge theories is related to their natural connection to square roots of four-momenta scalar products $s_{i j}$, cf. Eq. (D.13). Since gauge scattering amplitudes have square-root singularities in $s_{i j}$, they become simple functions when written in terms of spinor products.

We will now explain how spinors are used to simplify the description of massless gauge vector bosons. We consider a gluon with momentum $k$ and gauge vector $b$. Its polarization vector can be written as

$$
\varepsilon_{\mu}^{ \pm}(k, b)= \pm \frac{\left\langle k \pm\left|\gamma_{\mu}\right| b \pm\right\rangle}{\sqrt{2}\langle b \mp \mid k \pm\rangle} .
$$

Using Fierz and charge-conjugation equations, see Table D.1, we find

$$
\begin{aligned}
\gamma^{\mu} \varepsilon_{\mu}^{+}(k, b) & =\frac{\sqrt{2}[|k-\rangle\langle b-|+| b+\rangle\langle k+|]}{\langle b k\rangle}, \\
\gamma^{\mu} \varepsilon_{\mu}^{-}(k, b) & =\frac{\sqrt{2}[|k+\rangle\langle b+|+| b-\rangle\langle k-|]}{[k b]} .
\end{aligned}
$$

Different choices of the vector $b$ correspond to different gauge choices; the corresponding polarization vectors differ by an amount proportional to the gluon momentum. Specifically,

$$
\begin{aligned}
& \varepsilon_{\mu}^{+}(k, b)-\varepsilon_{\mu}^{+}(k, c)=\frac{\left\langle k+\left|\gamma_{\mu}\right| b+\right\rangle}{\sqrt{2}\langle b k\rangle}-\frac{\left\langle k+\left|\gamma_{\mu}\right| c+\right\rangle}{\sqrt{2}\langle c k\rangle} \\
= & \frac{1}{\sqrt{2}\langle b k\rangle\langle c k\rangle}\left[\left\langle k+\left|\gamma_{\mu}\right| b+\right\rangle\langle c k\rangle-\left\langle k+\left|\gamma_{\mu}\right| c+\right\rangle\langle b k\rangle\right] \\
= & \frac{1}{\sqrt{2}\langle b k\rangle\langle c k\rangle}\left[\left\langle k+\left|\gamma_{\mu}\right| k+\right\rangle\langle c b\rangle\right]=\frac{\sqrt{2}\langle c b\rangle}{\langle b k\rangle\langle c k\rangle} k_{\mu}
\end{aligned}
$$




$$
\begin{aligned}
& \langle p q\rangle=\langle p-\mid q+\rangle, \quad[p q]=\langle p+\mid q-\rangle \\
& \left\langle p \pm\left|\gamma_{\mu}\right| p \pm\right\rangle=2 p_{\mu} \\
& \langle p+\mid q+\rangle=\langle p-\mid q-\rangle=\langle p p\rangle=[p p]=0 \\
& \langle p q\rangle=-\langle q p\rangle, \quad[p q]=-[q p] \\
& 2|p \pm\rangle\langle q \pm|=\frac{1}{2}\left(1 \pm \gamma_{5}\right) \gamma^{\mu}\left\langle q \pm\left|\gamma_{\mu}\right| p \pm\right\rangle \\
& \langle p q\rangle^{*}=-\operatorname{sign}(p \cdot q)[p q]=\operatorname{sign}(p \cdot q)[q p] \\
& |\langle p q\rangle|^{2}=\langle p q\rangle\langle p q\rangle^{*}=2|p \cdot q| \equiv\left|s_{p q}\right| \\
& \langle p q\rangle[q p]=2 p \cdot q \equiv s_{p q} \\
& \left\langle p \pm\left|\gamma_{\mu_{1}} \ldots \gamma_{\mu_{2 n+1}}\right| q \pm\right\rangle=\left\langle q \mp\left|\gamma_{\mu_{2 n+1}} \ldots \gamma_{\mu_{1}}\right| p \mp\right\rangle \\
& \left\langle p \pm\left|\gamma_{\mu_{1}} \ldots \gamma_{\mu_{2 n}}\right| q \mp\right\rangle=-\left\langle q \pm\left|\gamma_{\mu_{2 n}} \ldots \gamma_{\mu_{1}}\right| p \mp\right\rangle \\
& \left\langle a+\left|\gamma_{\mu}\right| b+\right\rangle\left\langle c-\left|\gamma^{\mu}\right| d-\right\rangle=2[a d]\langle c b\rangle, \quad(\text { Fierz) } \\
& \left\langle a \pm\left|\gamma^{\mu}\right| b \pm\right\rangle \gamma_{\mu}=2[|a \mp\rangle\langle b \mp|+| b \pm\rangle\langle a \pm|], \quad \text { (Fierz + Charge conjugation) } \\
& \langle a b\rangle\langle c d\rangle=\langle a d\rangle\langle c b\rangle+\langle a c\rangle\langle b d\rangle . \quad \text { (Schouten) }
\end{aligned}
$$

Table D.1: A summary of relations valid for massless spinors.

where we have used Eq. (D.15).

\section{Three point vertices}

We will now calculate the amplitudes for the scattering of three massless partons; these will be important building blocks for the calculation of loop diagrams using spinor helicity techniques. By direct insertion of the colorordered Feynman rules, Fig. 6.1, we have

$$
\begin{aligned}
-i m_{3}\left(\bar{q}_{1}^{-}, q_{2}^{+}, g_{3}^{+}\right) & =-\frac{[23]\langle b 1\rangle}{\langle b 3\rangle}=-\frac{[23]^{2}}{[12]}, \\
-i m_{3}\left(\bar{q}_{1}^{-}, q_{2}^{+}, g_{3}^{-}\right) & =-\frac{[2 b]\langle 31\rangle}{[3 b]}=-\frac{\langle 31\rangle^{2}}{\langle 12\rangle} .
\end{aligned}
$$

The last steps follow from the momentum conservation which allows us to write

$$
\langle b 1\rangle[12]+\langle b 3\rangle[32]=0
$$

and obtain $\langle b 1\rangle /\langle b 3\rangle=[23] /[12]$. Using Eq. (D.23) and similar equations allows to remove all the dependence on the auxiliary vector $b$ in Eq. (D.22).

In a similar fashion for the $g g g$ amplitudes we have that, (choosing the gauge vector $b$ the same for all polarizations),

$$
\begin{aligned}
-i m_{3}\left(g_{1}^{-}, g_{2}^{+}, g_{3}^{+}\right) & =\sqrt{2}\left[\varepsilon_{1}^{-} \cdot \varepsilon_{2}^{+} \varepsilon_{3}^{+} \cdot p_{1}-\varepsilon_{3}^{+} \cdot \varepsilon_{1}^{-} \varepsilon_{2}^{+} \cdot p_{1}\right] \\
& =-\frac{\langle 1 b\rangle^{2}[23]}{\langle b 2\rangle\langle b 3\rangle}=-\frac{[23]^{3}}{[12][31]},
\end{aligned}
$$




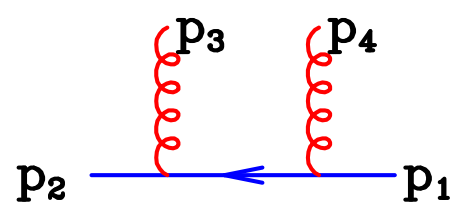

(a)

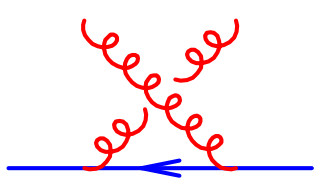

(b)

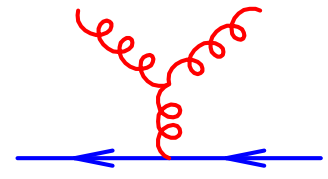

(c)

Figure D.1: Feynman diagrams for the process $0 \rightarrow \bar{q} q g g$.

$$
\begin{aligned}
-i m_{3}\left(g_{1}^{-}, g_{2}^{+}, g_{3}^{-}\right) & =\sqrt{2}\left[\varepsilon_{3}^{-} \cdot \varepsilon_{2}^{+} \varepsilon_{1}^{-} \cdot p_{2}-\varepsilon_{1}^{-} \cdot \varepsilon_{2}^{+} \varepsilon_{3}^{-} \cdot p_{1}\right] \\
& =\frac{[2 b]^{2}}{[1 b][b 2][3 b]}[\langle 12\rangle\langle 3 b\rangle-\langle 1 b\rangle\langle 32\rangle] \\
& =-\frac{[2 b]^{2}\langle 13\rangle}{[1 b][3 b]}=\frac{\langle 31\rangle^{3}}{\langle 12\rangle\langle 23\rangle} .
\end{aligned}
$$

In deriving these results we have used the expressions for the polarization vectors in terms of spinors, Eqs. (D.19-D.20), the Schouten identity, Eq. (D.17), and momentum conservation. Note that Eqs. (D.24,D.25) have the characteristic form of the maximal-helicity-violating (MHV) amplitudes [82]. These threepoint vertices can only be defined for complex momenta, since we have

$$
p_{1} \cdot p_{2}=p_{2} \cdot p_{3}=p_{3} \cdot p_{1}=0 .
$$

Thus, for example, we have that in the first equation in Eq. (D.22), $\langle 12\rangle=$ $\langle 23\rangle=\langle 31\rangle=0$ so that Eq. (D.26) is satisfied. For complex momenta this does not imply that [12], [23] or [31] are equal to zero.

Note also that there are simple relations between the $\bar{q} q g$ and $g g g$ amplitudes that can be derived using supersymmetry relations [82],

$$
\begin{aligned}
& m_{3}\left(g_{1}^{-}, g_{2}^{+}, g_{3}^{-}\right)=-m_{3}\left(\bar{q}_{1}^{-}, q_{2}^{+}, g_{3}^{-}\right) \frac{\langle 31\rangle}{\langle 23\rangle}, \\
& m_{3}\left(g_{1}^{-}, g_{2}^{+}, g_{3}^{+}\right)=+m_{3}\left(\bar{q}_{1}^{-}, q_{2}^{+}, g_{3}^{+}\right) \frac{[23]}{[31]} .
\end{aligned}
$$

The quark-gluon scattering process

To further illustrate how spinor techniques should be used at tree-level, we consider the process shown in Fig. (D.1), $\bar{q} q g g$ scattering. The momenta are labelled according to,

$$
0 \rightarrow \bar{q}\left(p_{1}\right)+q\left(p_{2}\right)+g\left(p_{3}\right)+g\left(p_{4}\right) .
$$

We first decompose the amplitude in terms of color ordered sub-amplitudes 
that are separately gauge invariant,

$$
\begin{aligned}
\mathcal{A}_{4}^{\text {tree }}\left(\bar{q}_{1}^{h_{1}}, q_{2}^{h_{2}}, g_{3}^{h_{3}}, g_{4}^{h_{4}}\right) & =g_{s}^{2}\left[\left(T^{a_{3}} T^{a_{4}}\right)_{i_{2} \bar{\imath}_{1}} m_{4}\left(\bar{q}_{1}^{h_{1}}, q_{2}^{h_{2}}, g_{3}^{h_{3}}, g_{4}^{h_{4}}\right)\right. \\
& \left.+\left(T^{a_{4}} T^{a_{3}}\right)_{i_{2} \bar{\imath}_{1}} m_{4}\left(\bar{q}_{1}^{h_{1}}, q_{2}^{h_{2}}, g_{4}^{h_{4}}, g_{3}^{h_{3}}\right)\right]
\end{aligned}
$$

This decomposition is obvious for the (non-abelian) diagrams $(a)$ and $(b)$ in Fig. (D.1). The diagram containing the triple-gluon vertex contributes to both $m_{4}\left(\bar{q}_{1}, q_{2}, g_{3}, g_{4}\right)$ and $m_{4}\left(\bar{q}_{1}, q_{2}, g_{4}, g_{3}\right)$ (with opposite signs) due to the color algebra relation,

$$
f^{a b c} T^{c}=-\frac{i}{\sqrt{2}}\left[T^{a}, T^{b}\right] .
$$

We take a negative helicity quark line and compute $m_{4}\left(\bar{q}_{1}, q_{2}, g_{3}, g_{4}\right)$ only. Because of the color factor, diagram (b) does not contribute to this color stripped amplitude and the other two diagrams are given by

$$
\begin{aligned}
& m_{4}^{(a)}=\frac{-i}{2}\left\langle 2\left|\not_{3} \frac{\left(\not p_{2}+\not \not_{3}\right)}{\langle 32\rangle[23]} \not_{4}\right| 1\right], \\
& m_{4}^{(c)}=\frac{-i}{\langle 34\rangle[43]}\left[\varepsilon_{3} \cdot \varepsilon_{4}\left\langle 2\left|\not \not_{4}\right| 1\right]+\varepsilon_{4} \cdot p_{3}\left\langle 2\left|\not_{3}\right| 1\right]-\varepsilon_{3} \cdot p_{4}\left\langle 2\left|\not_{4}\right| 1\right]\right] .
\end{aligned}
$$

At this point the calculation can be greatly simplified by an astute choice of gauge vectors $b_{3}$ and $b_{4}$, cf. Eq. (D.19). When the helicities of the two gluons are the same, we shall choose the two reference momenta $b_{3}, b_{4}$ to be the same; it then follows that $\varepsilon_{3} \cdot \varepsilon_{4}=0$. For the positive helicity case we choose $b_{3}=b_{4}=p_{2}$ so that,

$$
\langle 2| \not_{3}{ }^{+}=\langle 2| \not_{4}{ }^{+}=0 .
$$

We thus see that when both gluons have positive helicity, the amplitude vanishes $m_{4}\left(\bar{q}_{1}^{+}, q_{2}^{-}, g_{3}^{+}, g_{4}^{+}\right)=0$. Similarly it is easy to show that $m_{4}\left(\bar{q}_{1}^{+}, q_{2}^{-}, g_{3}^{-}, g_{4}^{-}\right)=0$ by choosing $b_{3}=b_{4}=p_{1}$.

The remaining helicity combination, when the gluons have opposite helicities, is most simple to compute by choosing $b_{3}=p_{4}$ and $b_{4}=p_{3}$. In that case we have the simplification,

$$
\varepsilon_{3} \cdot \varepsilon_{4}=\varepsilon_{3} \cdot p_{4}=\varepsilon_{4} \cdot p_{3}=0 .
$$

We again find that the contribution from diagram $(c)$ vanishes and only diagram (a) remains. This is a remarkable result: we have computed the quark gluon scattering matrix element in a non-Abelian theory, with no net contribution from the diagram involving the three gluon vertex. Its effect is completely fixed by gauge invariance. Completing the calculation we find from Eq. (D.31),

$$
-i m_{4}\left(\bar{q}_{1}^{+}, q_{2}^{-}, g_{3}^{+}, g_{4}^{-}\right)=\frac{\langle 24\rangle^{2}[31]}{\langle 32\rangle\langle 43\rangle[43]} .
$$

By multiplying top and bottom by $[31]^{2}$ and using momentum conservation we can put this in a simpler form,

$$
-i m_{4}\left(\bar{q}_{1}^{+}, q_{2}^{-}, g_{3}^{+}, g_{4}^{-}\right)=\frac{[31]^{3}}{[34][41][12]} .
$$


Similarly, the result for the opposite helicity choice is,

$$
-i m_{4}\left(\bar{q}_{1}^{+}, q_{2}^{-}, g_{3}^{-}, g_{4}^{+}\right)=-\frac{[41]^{2}[24]}{[23][34][21]} .
$$

Finally, the non-zero amplitudes for $m_{4}\left(\bar{q}_{1}^{+}, q_{2}^{-}, g_{4}, g_{3}\right)$ can be obtained by Bose symmetry (interchanging 3 and 4 ),

$$
\begin{aligned}
& -i m_{4}\left(\bar{q}_{1}^{+}, q_{2}^{-}, g_{4}^{+}, g_{3}^{-}\right)=\frac{[41]^{3}}{[43][31][12]}, \\
& -i m_{4}\left(\bar{q}_{1}^{+}, q_{2}^{-}, g_{4}^{-}, g_{3}^{+}\right)=-\frac{[31]^{2}[23]}{[24][43][21]},
\end{aligned}
$$

and parity invariance of the strong interactions means that,

$$
m_{4}\left(\bar{q}_{1}^{h_{1}}, q_{2}^{h_{2}}, g_{3}^{h_{3}}, g_{4}^{h_{4}}\right)=m_{4}^{*}\left(\bar{q}_{1}^{-h_{1}}, q_{2}^{-h_{2}}, g_{3}^{-h_{3}}, g_{4}^{-h_{4}}\right) \text {. }
$$

Amplitude for four quark scattering

In a similar way, the amplitude for four-quark scattering can easily be written down,

$$
\mathcal{B}^{\text {tree }}\left(\bar{q}_{1}, q_{2}, \bar{Q}_{3}, Q_{4}\right)=g_{s}^{2}\left[\delta_{i_{2} \bar{\imath}_{1}} \delta_{i_{4} \bar{\imath}_{3}}-\frac{1}{N_{c}} \delta_{i_{2} \bar{\imath}_{1}} \delta_{i_{4} \bar{\imath}_{3}}\right] m_{4}\left(\bar{q}_{1}, q_{2}, \bar{Q}_{3}, Q_{4}\right),
$$

where

$$
\begin{aligned}
-i m_{4}\left(\bar{q}_{1}^{+}, q_{2}^{-}, \bar{Q}_{3}^{+}, Q_{4}^{-}\right) & =-\frac{\left\langle 4\left|\gamma^{\mu}\right| 3\right]\left\langle 2\left|\gamma_{\mu}\right| 1\right]}{2 s_{12}}=-\frac{\langle 42\rangle[13]}{\langle 12\rangle[21]} \\
& =-\frac{\langle 42\rangle[13]^{2}}{[13]\langle 12\rangle[21]}=\frac{[13]^{2}}{[43][21]} .
\end{aligned}
$$

In deriving the last line of Eq. (D.40) we have used momentum conservation $[13]\langle 12\rangle=-\langle 2|\not| 3]=\langle 2|\not| 3| 3]=-[43]\langle 42\rangle$.

\section{Appendix E. Results for selected scalar integrals}

In this Appendix, we present scalar integrals that are needed for the computation of $q \bar{q} g g$ primitive amplitude discussed in Section 9.6. In all integrals, we neglect $\mathcal{O}(\epsilon)$ terms.

The result for the box integral with all external lines light-like is,

$$
\begin{aligned}
& I_{4}\left(0,0,0,0 ; s_{12}, s_{23} ; 0,0,0,0\right)=\frac{\mu^{2 \epsilon}}{s_{12} s_{23}} \\
& \times\left[\frac{2}{\epsilon^{2}}\left(\left(-s_{12}-i \varepsilon\right)^{-\epsilon}+\left(-s_{23}-i \varepsilon\right)^{-\epsilon}\right)-\ln ^{2}\left(\frac{-s_{12}-i \varepsilon}{-s_{23}-i \varepsilon}\right)-\pi^{2}\right]+\mathcal{O}(\epsilon) .
\end{aligned}
$$


The result for a triangle integral with two massless external lines is,

$$
\begin{aligned}
& I_{3}\left(0,0, p^{2} ; 0,0,0\right)=\frac{\mu^{2 \epsilon}}{\epsilon^{2}}\left(\frac{\left(-p^{2}-i \varepsilon\right)^{-\epsilon}}{p^{2}}\right) \\
& =\frac{1}{p^{2}}\left(\frac{1}{\epsilon^{2}}+\frac{1}{\epsilon} \ln \left(\frac{\mu^{2}}{-p^{2}-i \varepsilon}\right)+\frac{1}{2} \ln ^{2}\left(\frac{\mu^{2}}{-p^{2}-i \varepsilon}\right)\right)+\mathcal{O}(\epsilon) .
\end{aligned}
$$

Lastly, the result for the bubble integral is

$$
\begin{aligned}
I_{2}\left(p^{2} ; 0,0\right) & =\left(\frac{\mu^{2}}{-p^{2}-i \varepsilon}\right)^{\epsilon}\left(\frac{1}{\epsilon}+2\right) \\
& =\frac{1}{\epsilon}+\ln \left(\frac{\mu^{2}}{-p^{2}-i \varepsilon}\right)+2+\mathcal{O}(\epsilon) .
\end{aligned}
$$




\section{References}

[1] NLO Multileg Working Group Collaboration, Z. Bern et. al., The NLO multileg working group: Summary report, 0803.0494.

[2] A. Bredenstein, A. Denner, S. Dittmaier, and S. Pozzorini, NLO QCD corrections to $p p \rightarrow t$ anti- $t b$ anti- $b+X$ at the LHC, Phys. Rev. Lett. 103 (2009) 012002, [0905.0110].

[3] A. Bredenstein, A. Denner, S. Dittmaier, and S. Pozzorini, $N L O Q C D$ corrections to top anti-top bottom anti-bottom production at the LHC: 2. full hadronic results, JHEP 1003 (2010) 021, [1001.4006].

[4] G. Bevilacqua, M. Czakon, C. G. Papadopoulos, R. Pittau, and M. Worek, Assault on the NLO Wishlist: $p p \rightarrow t t$ bb, JHEP 09 (2009) 109, [0907. 4723].

[5] C. Berger, Z. Bern, L. J. Dixon, F. Febres Cordero, D. Forde, et. al., Precise Predictions for $W+3$ Jet Production at Hadron Colliders, Phys.Rev.Lett. 102 (2009) 222001, [0902.2760].

[6] R. K. Ellis, K. Melnikov, and G. Zanderighi, W+3 jet production at the Tevatron, Phys. Rev. D80 (2009) 094002, [0906.1445].

[7] C. Berger, Z. Bern, L. J. Dixon, F. Febres Cordero, D. Forde, et. al., Next-to-Leading Order QCD Predictions for W+3-Jet Distributions at Hadron Colliders, Phys.Rev. D80 (2009) 074036, [0907.1984].

[8] K. Melnikov and G. Zanderighi, W+3 jet production at the LHC as a signal or background, Phys. Rev. D81 (2010) 074025, [0910.3671].

[9] C. Berger, Z. Bern, L. J. Dixon, F. Cordero, D. Forde, et. al., Next-to-Leading Order QCD Predictions for Z, $\gamma^{*}+3$-Jet Distributions at the Tevatron, Phys.Rev. D82 (2010) 074002, [1004.1659].

[10] R. Frederix, S. Frixione, K. Melnikov, and G. Zanderighi, NLO QCD corrections to five-jet production at LEP and the extraction of alphas(MZ), JHEP 11 (2010) 050, [1008.5313].

[11] T. Melia, K. Melnikov, R. Rontsch, and G. Zanderighi, Next-to-leading order QCD predictions for $W^{+} W^{+} j j$ production at the LHC, JHEP 1012 (2010) 053, [1007.5313].

[12] A. Denner, S. Dittmaier, S. Kallweit, and S. Pozzorini, NLO QCD corrections to $W W b b$ production at hadron colliders, Phys.Rev.Lett. 106 (2011) 052001, [1012.3975].

[13] G. Bevilacqua, M. Czakon, C. Papadopoulos, and M. Worek, Dominant QCD Backgrounds in Higgs Boson Analyses at the LHC: A Study of pp $\rightarrow t$ anti- $t+2$ jets at Next-To-Leading Order, Phys.Rev.Lett. 104 (2010) 162002, [1002.4009]. 
[14] G. Bevilacqua, M. Czakon, A. van Hameren, C. G. Papadopoulos, and M. Worek, Complete off-shell effects in top quark pair hadroproduction with leptonic decay at next-to-leading order, JHEP 1102 (2011) 083, [1012.4230].

[15] T. Melia, K. Melnikov, R. Rontsch, and G. Zanderighi, NLO QCD corrections for $W^{+} W^{-}$pair production in association with two jets at hadron colliders, 1104.2327.

[16] N. Greiner, A. Guffanti, T. Reiter, and J. Reuter, NLO QCD corrections to the production of two bottom-antibottom pairs at the LHC, 1105.3624.

[17] C. Berger, Z. Bern, L. J. Dixon, F. Cordero, D. Forde, et. al., Precise Predictions for $W+4$ Jet Production at the Large Hadron Collider, Phys.Rev.Lett. 106 (2011) 092001, [1009.2338].

[18] H. Ita, Z. Bern, L. J. Dixon, F. Febres Cordero, D. A. Kosower and D. Maitre, Phys. Rev. D 85, 031501 (2012) [1108.2229].

[19] Z. Bern, L. J. Dixon, and D. A. Kosower, One-loop amplitudes for e+ eto four partons, Nucl. Phys. B513 (1998) 3-86, [hep-ph/9708239].

[20] R. Britto, F. Cachazo, and B. Feng, Generalized unitarity and one-loop amplitudes in $N=4$ super-Yang-Mills, Nucl. Phys. B725 (2005) 275-305, [hep-th/0412103].

[21] G. Ossola, C. G. Papadopoulos, and R. Pittau, Reducing full one-loop amplitudes to scalar integrals at the integrand level, Nucl. Phys. B763 (2007) 147-169, [hep-ph/0609007].

[22] R. K. Ellis, W. Giele, and Z. Kunszt, A Numerical Unitarity Formalism for Evaluating One-Loop Amplitudes, JHEP 0803 (2008) 003, [0708.2398].

[23] W. T. Giele, Z. Kunszt, and K. Melnikov, Full one-loop amplitudes from tree amplitudes, JHEP 04 (2008) 049, [0801.2237].

[24] R. K. Ellis, W. T. Giele, Z. Kunszt, and K. Melnikov, Masses, fermions and generalized D-dimensional unitarity, Nucl.Phys. B822 (2009) 270-282, [0806.3467].

[25] C. F. Berger and D. Forde, Multi-Parton Scattering Amplitudes via On-Shell Methods, Ann.Rev.Nucl.Part.Sci. (2009) [0912.3534].

[26] R. Britto, Loop amplitudes in gauge theories: modern analytic approaches, 1012.4493.

[27] J. S. Schwinger, Gauge Invariance and Mass. 2., Phys.Rev. 128 (1962) $2425-2429$. 
[28] F. A. Berends and W. Giele, The Six Gluon Process as an Example of Weyl-Van Der Waerden Spinor Calculus, Nucl. Phys. B294 (1987) 700.

[29] D. Forde, Direct extraction of one-loop integral coefficients, Phys. Rev. D75 (2007) 125019, [0704.1835].

[30] S. Badger, Direct Extraction Of One Loop Rational Terms, JHEP 0901 (2009) 049, [0806.4600].

[31] P. Mastrolia, Double-Cut of Scattering Amplitudes and Stokes' Theorem, Phys.Lett. B678 (2009) 246-249, [0905.2909].

[32] S. Badger, N. Bjerrum-Bohr, and P. Vanhove, Simplicity in the Structure of QED and Gravity Amplitudes, JHEP 0902 (2009) 038, [0811.3405].

[33] D. E. Soper, Choosing integration points for QCD calculations by numerical integration, Phys.Rev. D64 (2001) 034018, [hep-ph/0103262].

[34] G. Passarino, An Approach toward the numerical evaluation of multiloop Feynman diagrams, Nucl.Phys. B619 (2001) 257-312, [hep-ph/0108252].

[35] A. Ferroglia, M. Passera, G. Passarino, and S. Uccirati, All purpose numerical evaluation of one loop multileg Feynman diagrams, Nucl.Phys. B650 (2003) 162-228, [hep-ph/0209219].

[36] Z. Nagy and D. E. Soper, General subtraction method for numerical calculation of one loop QCD matrix elements, JHEP 0309 (2003) 055, [hep-ph/0308127].

[37] C. Anastasiou, S. Beerli, and A. Daleo, Evaluating multi-loop Feynman diagrams with infrared and threshold singularities numerically, JHEP 0705 (2007) 071, [hep-ph/0703282].

[38] A. Lazopoulos, K. Melnikov, and F. Petriello, QCD corrections to tri-boson production, Phys.Rev. D76 (2007) 014001, [hep-ph/0703273].

[39] Z. Nagy and D. E. Soper, Numerical integration of one-loop Feynman diagrams for N-photon amplitudes, Phys.Rev. D74 (2006) 093006, [hep-ph/0610028].

[40] W. Gong, Z. Nagy, and D. E. Soper, Direct numerical integration of one-loop Feynman diagrams for $N$-photon amplitudes, Phys.Rev. D79 (2009) 033005, [0812.3686].

[41] W. Kilian and T. Kleinschmidt, Numerical Evaluation of Feynman Loop Integrals by Reduction to Tree Graphs, 0912.3495.

[42] S. Becker, C. Reuschle, and S. Weinzierl, Numerical NLO QCD calculations, JHEP 1012 (2010) 013, [1010.4187]. 
[43] S. Becker, D. Goetz, C. Reuschle, C. Schwan, and S. Weinzierl, NLO results for five, six and seven jets in electron-positron annihilation, 1111.1733.

[44] S. Catani, T. Gleisberg, F. Krauss, G. Rodrigo, and J.-C. Winter, From loops to trees by-passing Feynman's theorem, JHEP 09 (2008) 065, [0804.3170].

[45] G. 't Hooft and M. J. G. Veltman, Regularization and Renormalization of Gauge Fields, Nucl. Phys. B44 (1972) 189-213.

[46] L. D. Landau, On analytic properties of vertex parts in quantum field theory, Nucl. Phys. 13 (1959) 181-192.

[47] T. Kinoshita, Mass singularities of Feynman amplitudes, J. Math. Phys. 3 (1962) 650-677.

[48] W. J. Marciano, Dimensional Regularization and Mass Singularities, Phys. Rev. D12 (1975) 3861.

[49] D. B. Melrose, Reduction of Feynman diagrams, Nuovo Cim. 40 (1965) 181-213.

[50] W. L. van Neerven and J. A. M. Vermaseren, Large loop integrals, Phys. Lett. B137 (1984) 241.

[51] Z. Bern, L. J. Dixon, and D. A. Kosower, Dimensionally Regulated One-Loop Integrals, Phys. Lett. B302 (1993) 299-308, [hep-ph/9212308].

[52] G. 't Hooft and M. Veltman, Scalar One Loop Integrals, Nucl.Phys. B153 (1979) 365-401.

[53] A. Denner, U. Nierste, and R. Scharf, A Compact expression for the scalar one loop four point function, Nucl. Phys. B367 (1991) 637-656.

[54] R. K. Ellis and G. Zanderighi, Scalar one-loop integrals for QCD, JHEP 02 (2008) 002, [0712.1851].

[55] R. K. Ellis and G. Zanderighi, "QCDLoop." http://qcdloop.fnal.gov.

[56] A. Denner and S. Dittmaier, Scalar one-loop 4-point integrals, Nucl. Phys. B844 (2011) 199-242, [1005. 2076].

[57] A. van Hameren, OneLOop: for the evaluation of one-loop scalar functions, 1007.4716.

[58] G. Passarino and M. J. G. Veltman, One Loop Corrections for $e^{+} e^{-}$ Annihilation Into $\mu^{+} \mu^{-}$in the Weinberg Model, Nucl. Phys. B160 (1979) 151. 
[59] P. Nogueira, Automatic Feynman graph generation, J. Comput. Phys. 105 (1993) 279-289.

[60] T. Hahn, Generating Feynman diagrams and amplitudes with FeynArts 3, Comput. Phys. Commun. 140 (2001) 418-431, [hep-ph/0012260].

[61] T. Binoth, J.-P. Guillet, G. Heinrich, E. Pilon, and T. Reiter, Golem95: A Numerical program to calculate one-loop tensor integrals with up to six external legs, Comput.Phys.Commun. 180 (2009) 2317-2330, [0810.0992].

[62] G. Cullen et. al., Recent Progress in the Golem Project, Nucl. Phys. Proc. Suppl. 205-206 (2010) 67-73, [1007.3580].

[63] A. Denner and S. Dittmaier, Reduction schemes for one-loop tensor integrals, Nucl. Phys. B734 (2006) 62-115, [hep-ph/0509141].

[64] W. Giele, E. Glover, and G. Zanderighi, Numerical evaluation of one-loop diagrams near exceptional momentum configurations, Nucl.Phys.Proc.Suppl. 135 (2004) 275-279, [hep-ph/0407016].

[65] G. J. van Oldenborgh and J. A. M. Vermaseren, New Algorithms for One Loop Integrals, Z. Phys. C46 (1990) 425-438.

[66] E. Byckling and K. Kajantie, Particle kinematics. Wiley, London and New York, 1973.

[67] R. K. Ellis, W. T. Giele, Z. Kunszt, K. Melnikov, and G. Zanderighi, One-loop amplitudes for $W^{+} 3$ jet production in hadron collisions, JHEP 01 (2009) 012, [0810.2762].

[68] A. Lazopoulos, Precise evaluation of the Cut Constructible part of one loop amplitudes within D-dimensional unitarity, 0911.5241.

[69] P. Mastrolia, G. Ossola, C. G. Papadopoulos, and R. Pittau, Optimizing the Reduction of One-Loop Amplitudes, JHEP 06 (2008) 030, [0803.3964].

[70] C. F. Berger et. al., An Automated Implementation of On-Shell Methods for One- Loop Amplitudes, Phys. Rev. D78 (2008) 036003, [0803.4180].

[71] K. Melnikov and M. Schulze, NLO QCD corrections to top quark pair production in association with one hard jet at hadron colliders, Nucl.Phys. B840 (2010) 129-159, [1004.3284].

[72] K. Melnikov and M. Schulze, NLO QCD corrections to top quark pair production and decay at hadron colliders, JHEP 08 (2009) 049, [0907.3090]. 
[73] Z. Bern, L. J. Dixon, D. C. Dunbar, and D. A. Kosower, Fusing gauge theory tree amplitudes into loop amplitudes, Nucl. Phys. B435 (1995) 59-101, [hep-ph/9409265].

[74] G. Ossola, C. G. Papadopoulos, and R. Pittau, On the Rational Terms of the one-loop amplitudes, JHEP 05 (2008) 004, [0802.1876].

[75] Z. Bern, A. De Freitas, L. J. Dixon, and H. L. Wong, Supersymmetric regularization, two-loop QCD amplitudes and coupling shifts, Phys. Rev. D66 (2002) 085002, [hep-ph/0202271].

[76] M. Garzelli, I. Malamos, and R. Pittau, Feynman rules for the rational part of the Electroweak 1-loop amplitudes in the $R_{\xi}$ gauge and in the Unitary gauge, 1009.4302.

[77] M. Garzelli, I. Malamos, and R. Pittau, Feynman rules for the rational part of the Electroweak 1-loop amplitudes, JHEP 1001 (2010) 040, [0910.3130].

[78] P. Draggiotis, M. Garzelli, C. Papadopoulos, and R. Pittau, Feynman Rules for the Rational Part of the QCD 1-loop amplitudes, JHEP 0904 (2009) 072, [0903.0356].

[79] Z. Bern, L. J. Dixon, and D. A. Kosower, Bootstrapping multi-parton loop amplitudes in QCD, Phys.Rev. D73 (2006) 065013, [hep-ph/0507005].

[80] C. F. Berger, Z. Bern, L. J. Dixon, D. Forde, and D. A. Kosower, Bootstrapping one-loop QCD amplitudes with general helicities, Phys. Rev. D74 (2006) 036009, [hep-ph/0604195].

[81] M. L. Mangano, The Color Structure of Gluon Emission, Nucl. Phys. B309 (1988) 461.

[82] M. L. Mangano and S. J. Parke, Multiparton amplitudes in gauge theories, Phys.Rept. 200 (1991) 301-367, [hep-th/0509223].

[83] L. J. Dixon, Calculating scattering amplitudes efficiently, hep-ph/9601359.

[84] Z. Bern, L. J. Dixon, and D. A. Kosower, One loop corrections to two quark three gluon amplitudes, Nucl.Phys. B437 (1995) 259-304, [hep-ph/9409393].

[85] Z. Bern, L. J. Dixon, and D. A. Kosower, Progress in one loop QCD computations, Ann.Rev.Nucl.Part.Sci. 46 (1996) 109-148, [hep-ph/9602280].

[86] F. A. Berends and W. T. Giele, Multiple Soft Gluon Radiation in Parton Processes, Nucl. Phys. B313 (1989) 595. 
[87] R. Kleiss and H. Kuijf, Multi - gluon cross-sections and five jet production at hadron colliders, Nucl. Phys. B312 (1989) 616.

[88] V. Del Duca, L. J. Dixon, and F. Maltoni, New color decompositions for gauge amplitudes at tree and loop level, Nucl. Phys. B571 (2000) 51-70, [hep-ph/9910563].

[89] W. Giele, private communication.

[90] Z. Bern, J. J. M. Carrasco, and H. Johansson, New Relations for Gauge-Theory Amplitudes, Phys. Rev. D78 (2008) 085011, [0805.3993].

[91] N. E. J. Bjerrum-Bohr, P. H. Damgaard, T. Sondergaard, and P. Vanhove, Monodromy and Jacobi-like Relations for Color-Ordered Amplitudes, JHEP 06 (2010) 003, [1003.2403].

[92] B. Feng, R. Huang, and Y. Jia, Gauge Amplitude Identities by On-shell Recursion Relation in S-matrix Program, Phys. Lett. B695 (2011) 350-353, [1004.3417].

[93] Y.-X. Chen, Y.-J. Du, and B. Feng, A Proof of the Explicit Minimal-basis Expansion of Tree Amplitudes in Gauge Field Theory, JHEP 02 (2011) 112, [1101.0009].

[94] W. Giele, Z. Kunszt, and J. Winter, Efficient Color-Dressed Calculation of Virtual Corrections, Nucl. Phys. B840 (2010) 214-270, [0911.1962].

[95] T. Gleisberg and S. Hoche, Comix, a new matrix element generator, JHEP 12 (2008) 039, [0808.3674].

[96] Z. Kunszt, A. Signer, and Z. Trocsanyi, Singular terms of helicity amplitudes at one loop in $Q C D$ and the soft limit of the cross-sections of multiparton processes, Nucl. Phys. B420 (1994) 550-564, [hep-ph/9401294].

[97] S. Catani, The singular behaviour of QCD amplitudes at two-loop order, Phys. Lett. B427 (1998) 161-171, [hep-ph/9802439].

[98] S. Catani, S. Dittmaier, and Z. Trocsanyi, One loop singular behavior of QCD and SUSY QCD amplitudes with massive partons, Phys.Lett. B500 (2001) 149-160, [hep-ph/0011222].

[99] C. Cheung and D. O'Connell, Amplitudes and Spinor-Helicity in Six Dimensions, JHEP 0907 (2009) 075, [0902.0981].

[100] S. Caron-Huot and D. O'Connell, Spinor Helicity and Dual Conformal Symmetry in Ten Dimensions, 1010.5487.

[101] J. Collins, Renormalization. Cambridge University Press, Cambridge, 1984 . 
[102] F. Berends and W. Giele, Recursive calculations for Processes with $n$ gluons, Nucl. Phys. B306 (1988) 759.

[103] K. Melnikov, M. Schulze, and A. Scharf, QCD corrections to top quark pair production in association with a photon at hadron colliders, Phys.Rev. D83 (2011) 074013, [1102.1967].

[104] R. Britto, F. Cachazo, B. Feng, and E. Witten, Direct Proof Of Tree-Level Recursion Relation In Yang-Mills Theory, Phys. Rev. Lett. 94 (2005) 181602, [hep-th/0501052].

[105] N. Arkani-Hamed and J. Kaplan, On Tree Amplitudes in Gauge Theory and Gravity, JHEP 04 (2008) 076, [0801.2385].

[106] S. Badger, R. Sattler, and V. Yundin, One-Loop Helicity Amplitudes for ttbar Production at Hadron Colliders, 1101.5947.

[107] D. J. Broadhurst, N. Gray, and K. Schilcher, Gauge invariant on-shell $Z(2)$ in $Q E D, Q C D$ and the effective field theory of a static quark, $Z$. Phys. C52 (1991) 111-122.

[108] N. Arkani-Hamed, F. Cachazo, and J. Kaplan, What is the Simplest Quantum Field Theory?, JHEP 1009 (2010) 016, [0808.1446].

[109] W. B. Kilgore, One-loop Integral Coefficients from Generalized Unitarity, 0711.5015.

[110] S. J. Parke and T. R. Taylor, An Amplitude for $n$ Gluon Scattering, Phys. Rev. Lett. 56 (1986) 2459.

[111] Z. Kunszt, A. Signer, and Z. Trocsanyi, One loop helicity amplitudes for all 2 to 2 processes in $Q C D$ and $N=1$ supersymmetric Yang-Mills theory, Nucl.Phys. B411 (1994) 397-442, [hep-ph/9305239].

[112] M. E. Peskin, Simplifying Multi-Jet QCD Computation, 1101.2414.

[113] J. M. Drummond, Hidden Simplicity of Gauge Theory Amplitudes, Class. Quant. Grav. 27 (2010) 214001, [1010.2418].

[114] E. N. Glover and C. Williams, One-Loop Gluonic Amplitudes from Single Unitarity Cuts, JHEP 0812 (2008) 067, [0810.2964].

[115] B. W. Lee, C. Quigg, and H. Thacker, Weak Interactions at Very High-Energies: The Role of the Higgs Boson Mass, Phys.Rev. D16 (1977) 1519.

[116] M. S. Chanowitz and M. K. Gaillard, The TeV Physics of Strongly Interacting W's and Z's, Nucl.Phys. B261 (1985) 379.

[117] J. R. Ellis, M. K. Gaillard, and D. V. Nanopoulos, A Phenomenological Profile of the Higgs Boson, Nucl.Phys. B106 (1976) 292. 
[118] R. K. Ellis, W. J. Stirling, and B. R. Webber, QCD and collider physics, Camb. Monogr. Part. Phys. Nucl. Phys. Cosmol. 8 (1996) 1-435.

[119] T. Binoth, J. Guillet, and G. Heinrich, Algebraic evaluation of rational polynomials in one-loop amplitudes, JHEP 0702 (2007) 013, [hep-ph/0609054].

[120] S. L. Adler, Axial vector vertex in spinor electrodynamics, Phys. Rev. 177 (1969) 2426-2438.

[121] J. S. Bell and R. Jackiw, A PCAC puzzle: piO $\rightarrow$ gamma gamma in the sigma model, Nuovo Cim. A60 (1969) 47-61.

[122] R. Pittau, Primary Feynman rules to calculate the epsilon-dimensional integrand of any 1-loop amplitude, 1111.4965.

[123] T. Binoth et. al., A proposal for a standard interface between Monte Carlo tools and one-loop programs, Comput. Phys. Commun. 181 (2010) $1612-1622$, [1001.1307].

[124] V. Hirschi et. al., Automation of one-loop QCD corrections, 1103.0621.

[125] A. van Hameren, C. G. Papadopoulos, and R. Pittau, Automated one-loop calculations: a proof of concept, JHEP 09 (2009) 106, [0903.4665].

[126] P. Mastrolia, G. Ossola, T. Reiter, and F. Tramontano, Scattering AMplitudes from Unitarity-based Reduction Algorithm at the Integrand-level, JHEP 08 (2010) 080, [1006.0710].

[127] J. Vermaseren, New features of FORM, math-ph/0010025.

[128] J. Alwall et. al., MadGraph/MadEvent v4: The New Web Generation, JHEP 09 (2007) 028, [0706.2334].

[129] D. H. Bailey, "A Portable High Performance Multiprecision Package." NASA Ames RNR Technical Report RNR-90-022, 1990.

[130] D. H. Bailey, "A Fortran-90 Based Multiprecision System." RNR Technical Report RNR-94-013, 1994.

[131] R. Pittau, Testing and improving the numerical accuracy of the NLO predictions, Comput. Phys. Commun. 181 (2010) 1941-1946, [1006.3773].

[132] W. T. Giele and G. Zanderighi, On the Numerical Evaluation of One-Loop Amplitudes: The Gluonic Case, JHEP 06 (2008) 038, [0805.2152].

[133] J.-C. Winter and W. T. Giele, Calculating gluon one-loop amplitudes numerically, 0902.0094. 
[134] A. Lazopoulos, Multi-gluon one-loop amplitudes numerically, 0812.2998.

[135] M. L. Mangano, M. Moretti, F. Piccinini, R. Pittau, and A. D. Polosa, ALPGEN, a generator for hard multiparton processes in hadronic collisions, JHEP 07 (2003) 001, [hep-ph/0206293].

[136] T. Gleisberg et. al., SHERPA 1.alpha, a proof-of-concept version, JHEP 02 (2004) 056, [hep-ph/0311263].

[137] CompHEP Collaboration, E. Boos et. al., CompHEP 4.4: Automatic computations from Lagrangians to events, Nucl. Instrum. Meth. $\mathbf{A 5 3 4}$ (2004) 250-259, [hep-ph/0403113].

[138] W. T. Giele and E. W. N. Glover, A calculational formalism for one-loop integrals, JHEP 04 (2004) 029, [hep-ph/0402152].

[139] I. Bierenbaum, S. Catani, P. Draggiotis, and G. Rodrigo, A Tree-Loop Duality Relation at Two Loops and Beyond, JHEP 10 (2010) 073, [1007.0194].

[140] F. A. Berends, R. Kleiss, P. De Causmaecker, R. Gastmans, and T. T. $\mathrm{Wu}$, Single Bremsstrahlung Processes in Gauge Theories, Phys.Lett. B103 (1981) 124.

[141] L. J. Dixon, J. M. Henn, J. Plefka, and T. Schuster, All tree-level amplitudes in massless QCD, JHEP 01 (2011) 035, [1010.3991].

[142] J. L. Bourjaily, Efficient Tree-Amplitudes in N=4: Automatic BCFW Recursion in Mathematica, 1011.2447.

[143] S. Badger, J. M. Campbell, and R. K. Ellis, QCD corrections to the hadronic production of a heavy quark pair and a $W$-boson including decay correlations, JHEP 1103 (2011) 027, [1011.6647].

[144] J. M. Campbell, R. K. Ellis, and C. Williams, Hadronic production of a Higgs boson and two jets at next-to-leading order, Phys.Rev. D81 (2010) 074023, [1001.4495].

[145] S. Caron-Huot, Loops and trees, 1007.3224.

[146] N. Arkani-Hamed, J. L. Bourjaily, F. Cachazo, S. Caron-Huot, and J. Trnka, The All-Loop Integrand For Scattering Amplitudes in Planar N=4 SYM, JHEP 1101 (2011) 041, [1008.2958].

[147] R. H. Boels, On BCFW shifts of integrands and integrals, JHEP 11 (2010) 113, [1008.3101].

[148] J. Gluza, K. Kajda, and D. A. Kosower, Towards a Basis for Planar Two-Loop Integrals, Phys.Rev. D83 (2011) 045012, [1009.0472]. 
[149] J. D. Bjorken and S. D. Drell, Relativistic quantum mechanics. McGraw-Hill, 1965.

[150] R. E. Cutkosky, Singularities and discontinuities of Feynman amplitudes, J. Math. Phys. 1 (1960) 429-433.

[151] V. Berestetskii, E. Lifschitz, and L. Pitaevsky, Quantum Electrodynamics. Butterworth-Heineman, 1982.

[152] R. J. Eden, P. V. Landshoff, D. I. Olive, and J. C. Polkinghorne, The Analytic S-Matrix. Cambridge at the University Press, 1966.

[153] K. Nishijima, Unitarity Condition and Anomalous Vertex Functions, Phys. Rev. 126 (1962) 852-860.

[154] Z. Xu, D.-H. Zhang, and L. Chang, Helicity Amplitudes for Multiple Bremsstrahlung in Massless Nonabelian Gauge Theories, Nucl. Phys. B291 (1987) 392.

[155] Z. Bern, L. J. Dixon, and D. A. Kosower, On-Shell Methods in Perturbative QCD, Annals Phys. 322 (2007) 1587-1634, [0704.2798]. 